\title{
Mechanisms of Neuroligin Function in Inhibitory Postsynaptic Differentiation
}

\author{
PhD Thesis \\ in partial fulfilment of the requirements \\ for the degree "Doctor of Philosophy (PhD)" \\ in the Neuroscience Program \\ at the Georg August University Göttingen, \\ Faculty of Biology
}

submitted by

Alexandros Poulopoulos

born in

Athens 
Dedicated to Chrisanthi 


\section{Declaration}

This thesis has been written independently and with no other sources and aids than quoted.

Alexandros Poulopoulos

Göttingen, March 27th, 2008 


\section{Acknowledgments}

Carrying out this work would not have been possible, let alone as fulfilling, had I not been fortunate enough to have the support of many people. I express my sincere gratitude towards my supervisor, Dr. Frédérique Varoqueaux, for her encouragement and guidance, for actively supporting independent thinking and for making the student-supervisor relationship an unlikely source of fun. I am indebted to Dr. Guido Meyer for being an invaluable guide in my first attempts at experimental design, implementation and interpretation, and a role model for scientific conduct. I am grateful to Prof. Nils Brose, head of my thesis committee, for his critical scientific input and, most importantly, for embracing me -as a colleague- in his lab, where he has created a uniquely stimulating environment of exchange of thoughts and know-how. I thank Dr. Evgeni Ponimaskin and Prof. Ralf Heinrich who, as members of my thesis committee, provided valuable input and reflections on the progress of my work.

The Göttingen Neuroscience Graduate Program and all of its constituents provided the ideal framework for academic development. I am grateful to Sandra Drube, Dr. Simone Cardoso de Oliveira, Dr. Michael Hörner and Dr. Steffen Burkhard and who were an omnipresent support team throughout the course of study. I am additionally grateful to Prof. Detlef Doenecke and the DFG's Graduirtenkolleg 521 for supporting my research and training. My colleagues in study were equally important educators as all were happy to share their understanding of science. I am fortunate to have had Lucian Medrihan, Felipe Opazo and Stephan Junek as colleagues, friends and flat-mates, with whom countless hours were spent contemplating on the next experiment, on the latest paper, on the nature of consciousness and on the next night out.

This study would have been markedly poorer and far less enjoyable had it not been for my colleagues in the department of Molecular Neurobiology of the Max Planck Institute of Experimental Medicine. I especially thank Klaus Hellmann for his rock-solid technical help. I have been very lucky to have worked alongside friends Dr. Benjamin Cooper, Noa Lipstein, Randi Rawson, Andrea Burgalossi, Kalina Dimova, Dr. Etienne Herzog, Dr. Wolf Jockusch and Dr. Jeong Seop Rhee who were both the science and the fun in everyday life in and out of the lab. I had the privilege of working with two exceptional students, loanna Bethani and Tolga 
Soykan -the latter joining the lab and being my colleague, friend and bench-mate, who dedicated themselves to our research and with whom we spent many a night with ambitious hypotheses or frustrated at the bench. I thank my external collaborators for graciously providing reagents and Prof. Ann Marie Craig and Dr. Yunhee Kang for sharing their valuable expertise and for making me feel at home during my stay in the Craig lab.

I am grateful to those in my undergraduate years at the University of Athens who were an influential part of my academic and student life. I am indebted to Dr. Spiros Efthimiopoulos and Dr. Angeliki Fotinopoulou who supervised my first steps in research and supported me with wholehearted advice for my future endeavors. I also thank Dr. Georgos Diallinas for his inspired lectures and many thoughtprovoking discussions. Reaching graduate school would not have been possible without the irreplaceable support of friends and colleagues Maria Billini, Soultana Stylianidou, Foteini Orfaniotou, Natalia Milogiannaki, Konstantinos Tsagarakis, Konstantinos Kagias, Dr. Areti Pantazopoulou and many more.

I feel the need to recognize the great importance of many grade-school and highschool teachers, most notable of which Erikkos Giakoumakis, whose profound influence guided me towards academia. I am grateful to Elias Psathas, Giorgos Diamantis and loannis Charitakis, friends from childhood who have been with me even from afar.

Most of all, I am indebted to my family who has graciously and lovingly provided for me throughout; to Dr. Cornelia Poulopoulou who, more than a life-long friend, colleague and, lately, collaborator, is a great mom; to Giorgos and Christos Poulopoulos; to my yiayia and guardian angel, Chrysanthi Poulopoulou, I dedicate this work with all my heart. 


\section{Introduction 9}

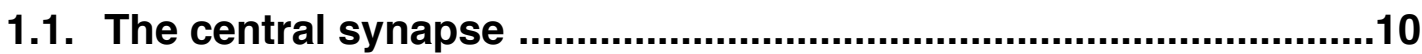

1.1.1. Synaptic transmission and signal integration 10

1.1.2. The active zone of presynaptic release 12

1.1.3. The postsynaptic receptor apparatus 14

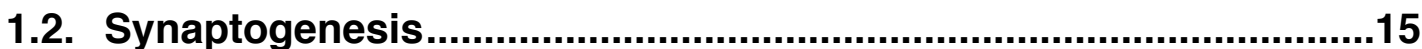

1.2.1. The paradigm of neuromuscular junction formation 16

1.2.2. Synaptogenesis in the central nervous system 18

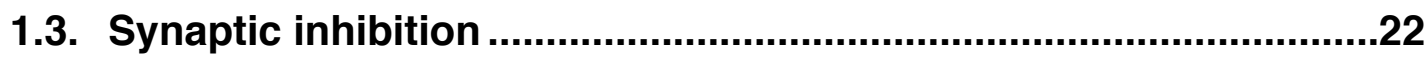

1.3.1. Glycine receptors 23

1.3.2. GABA receptors 24

1.3.3. Tonic and phasic inhibition 25

1.3.4. Inhibitory receptor clustering 27

1.4. Assembly mechanisms of the postsynaptic apparatus .................29

1.4.1. PDZ domain scaffolds 30

1.4.2. The Gephyrin scaffold 31

1.4.3. The Neuroligin family of adhesion proteins 35

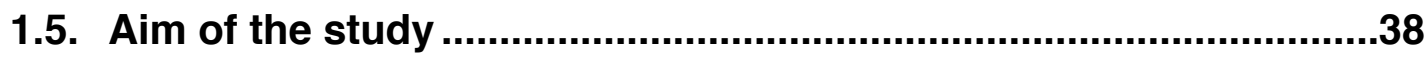

\section{Materials \& Methods 39}

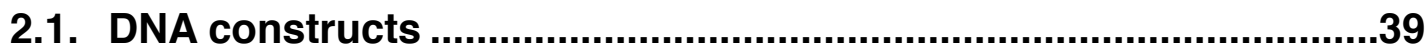

2.1.1. Yeast-two-hybrid plasmids 39

2.1.2. Expression plasmids $\quad 40$

2.1.3. Cloning of full-length DRP2 41

2.2. Antibodies \& labeling reagents .................................................42

2.2.1. List of primary antibodies and working dilutions 42

2.2.2. Conjugated antibodies and reagents 43 
2.2.3. Production of anti-DRP2 antisera 43

2.3. Yeast-two-hybrid assays .44

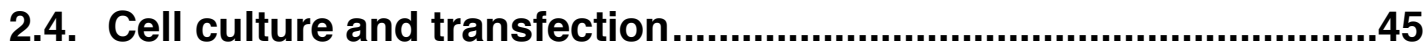

2.4.1. Cell-Line culture and transfection 45

2.4.2. Primary neuron culture 45

2.5. Recombinant protein binding assays ..........................................46

2.5.1. GST-fusion proteins 46

$\begin{array}{ll}\text { 2.5.2. Fc-fusion proteins } & 47\end{array}$

2.5.3. Co-immunoprecipitation in cell-lines 47

2.6. Crosslinking and identification of protein complexes ..................48

2.6.1. Preparation of crosslinked brain material 48

2.6.2. Preparations with in situ crosslinking of cell-line cultures 48

2.6.3. Preparations with in situ crosslinking of neuron cultures 48

2.6.4. Immunoprecipitation of crosslinked complexes 49

2.7. Synaptic plasma membrane preparation .......................................49

2.8. Immunolabeling and surface clustering assays ..........................50

2.8.1. Immunolabeling of neurons and transfected cell-lines 50

2.8.2. Acute surface clustering of transfected neurons and cell-lines $\quad 51$

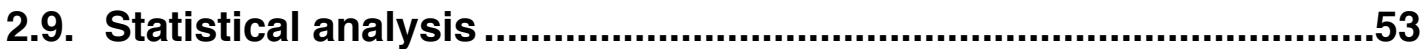

\section{Results 54}

3.1. Investigative analysis of novel cytosolic interaction partners of Neuroligins

3.1.1. Yeast-two-hybrid analysis of putative cytosolic interaction partners of Neuroligin 2

3.1.2. Investigative Neuroligin 2 protein interaction assays with putative interaction partners

3.2. Study on the role of Neuroligin 2 in the assembly of the inhibitory postsynaptic scaffold

3.2.1. Gephyrin is a putative cytosolic binding partner of Neuroligins 71 
3.2.2. Neuroligin 2 and Gephyrin form a complex in brain and heterologous cells

3.2.3. Identification of a novel Gephyrin-binding motif conserved in Neuroligins

3.2.4. Neuroligins are recruited to Collybistin-induced Gephyrin membrane aggregates in heterologous cells

3.2.5. The Neuroligin 2-Gephyrin interaction contributes to their coclustering in neurons

3.2.6. Neuroligin 2 activates membrane association of native Collybistin86

3.2.7. Gephyrin recruitment to somatic postsynaptic sites is disrupted in Neuroligin 2-deficient neurons

3.2.8. Involvement of Neuroligin 2 in the recruitment of GABAA receptors to postsynaptic sites

3.3. Study on the nature and assembly of Neuroligin oligomers .92

3.3.1. Neuroligins appear as dimers on the neuronal surface

3.3.2. Neuroligin dimers can be heteromeric and have specific paralogue combinations

3.3.3. Neuroligin monomers are retained in the early secretory pathway via a transmembrane retention signal

\section{Discussion 101}

4.1. Functional unit of Neuroligins: the dimer ...................................101

4.2. Neuroligin dimers: assembly and signaling...............................108

4.3. Postsynaptic functions of the Neuroligin family: receptors, scaffolds and regulators

4.4. Mechanistic role of Neuroligin 2 at the inhibitory postsynapse..123

4.5. Principles of Neuroligin function and dysfunction 139

\section{Summary 144}

6. References $\mathbf{1 4 5}$ 


\section{Introduction}

Taking on the role of a grand coordinator, the Central Nervous System (CNS) functions to orchestrate corporeal function and development, integrating and processing information from other bodily systems and evoking responses through signaling. More remarkable however are the functions of the CNS that give rise to behavior, involving information processing from the environment and orchestrating the body's acute associated responses. Far beyond "remarkable" -indeed reaching the limits of our understanding of nature- is the postulation of the 'encephalocentric theory' of the mind by Alcmaeon and Hippocrates (Crivellato \& Ribatti, 2007), arguing that 'the brain is the seat of the mind', in other terms that the brain has the capacity to give rise to consciousness, a phenomenon better left understood through the first-person experience of the reader.

Our current understanding of the CNS in biological terms testifies that, despite the unique characteristics of the phenomena which it mediates, the underlying principles of its function are based on the same fundamental processes as observed in liver, muscle, epithelia, or any given eukaryotic cell system. Ramón y Cajal was first to dispel the notion that unique biological phenomena govern brain function by proposing the neuron doctrine.

We now understand that, while neurons principally function as do, for instance, hepatocytes, the complexity of neuronal systems is many orders of magnitude greater than that of other cell systems. It is postulated that it is exactly this extraordinary level of complexity that is the basis for the brain's unique functions, including those that pertain to the mind (Searle, 2005). As it is complexity, rather than novelty, which underlies brain function, a reductionist approach, drawing from the accumulating knowledge on the biology of the cell, would be well-suited as a starting point in attempting to unravel what appears as a Gordian knot of neuronal ensembles. 


\subsection{The central synapse}

The human brain is composed of one-hundred billion neurons, the cell-types that handle information. These neurons functionally connect to each other through onequadrillion synapses, forming expansive networks that can relay and process information. This networking of information-handling units has led to the proposal of the computational model of brain function (Minsky, 1986). In this view, the primary functional property of neurons is connectivity. They are highly differentiated cells that specialize in forming synapses, the units of neuronal connectivity. Understanding synapse function thus appears critical in order to grasp the higher level characteristics of brain function.

\subsubsection{Synaptic transmission and signal integration}

Neurons encode information through the electric excitability of their plasma membrane. A neuron at rest sustains a difference in charge between the cytoplasmic and extracellular sides of its membrane, typically creating an electric potential difference of $-70 \mathrm{mV}$. The neuron is excited when a change in membrane conductance, mediated by ion channel gating, causes a flux of charge that changes the membrane potential. Through positive feedback mechanisms, depolarization typically peaking at $+50 \mathrm{mV}$ propagates throughout the neuron as $\mathrm{Ca}^{2+}$ or $\mathrm{Na}^{+}$waves depending on the neuronal compartment. It is this transition between resting and excited electrical states of the neuronal membrane that gives the neuron its capacity to encode information.

Neurons transmit their electrically-encoded information to other neurons through specialized transcellular junctions termed 'synapses'. Synapses function as computational transistors in their capacity to convey depolarizations from one neuron to another in a regulated manner. They can be categorized into two groups that differ principally both in structure and in function. Electrical synapses, or gapjunctions, are symmetric contacts between two neurons. These neurons are electrically coupled as gap junction contacts are direct conductors of ionic charge allowing changes in membrane potential to spread to both neurons (reviewed by Connors \& Long, 2004). Chemical synapses are contacts between neurons that 
transmit electric signals through an indirect mechanism that is directionally selective. Chemical synapses modify the electrical properties of the transmitted signal giving them the capacity to interpret a given input. It is this property that gives chemical synapses a principal role in the computational power of neuronal networks and, not surprisingly, chemical synapses represent the bulk of synaptic contacts in the brain.

Chemical synapses are asymmetric, mediating unidirectional transmission. It is the electric activity of one neuron in the junction (the presynaptic neuron) that provides input for the synapse, and the other neuron (the postsynaptic neuron) that is the recipient of the synaptic signal. Depolarization waves carried by voltage-gated $\mathrm{Na}^{+}$ and $\mathrm{K}^{+}$channels in the form of action potentials propagate throughout the output component of the presynaptic neuron. As the $\mathrm{Na}^{+}$depolarization front reaches the site of a chemical synapse, typically on a specialized neuronal process termed the axon, voltage-gated $\mathrm{Ca}^{2+}$ channels open, leading to the $\mathrm{Ca}^{2+}$-dependent release of compounds termed 'neurotransmitters' into the extracellular space between presynaptic and postsynaptic cells termed the 'synaptic cleft'. Thus in the first step of synaptic transmission, an electrical signal in the presynaptic cell is translated into a chemical signal extracellularly.

At the postsynaptic side, or the receiving end of a chemical synapse, receptor proteins sensitive to the transmitter released by the presynaptic side are activated, eliciting a postsynaptic response. In the case of fast synaptic transmission, ligandgated ion channels change the ion conductance of the postsynaptic membrane translating the chemical signal in the synaptic cleft back to an electric signal in the postsynaptic neuron. A crucial property that arises from this indirect transmission mechanism is that, while the presynaptic signal is digital in the form of stereotyped action potentials, the resulting postsynaptic signal is analogue, or graded in intensity. The strength of the postsynaptic signal will be determined by the specific properties of the transmitting synapse.

Importantly, even the qualitative property of the postsynaptic signal is determined by the synapse. Depending on the ion selectivity of the postsynaptic receptor channels, a postsynaptic response can be depolarizing or hyperpolarizing. Cation selective ligand-gated channels mediate depolarizing responses as they elicit in- 
flux of positive charge. As depolarization perturbs the neuronal membrane from its resting state, these synapses are termed 'excitatory' and in the mammalian brain are typically gated by the transmitter glutamate. Synapses which have $\mathrm{Cl}^{-}-$selective channels mediate the influx of negative charge in the mature CNS, causing hyperpolarization. As hyperpolarizing hinders excitation, these synapses are termed 'inhibitory' and are typically gated by the transmitters glycine and $\mathrm{Y}$ aminobutyric acid (GABA).

Each synapse thus contributes an electrical input of a certain sign and intensity to the postsynaptic neuron. The receiving component of the neuron, typically the somatodendritic compartment (i.e the neuronal membrane excluding the axon), integrates synaptic input by way of diffusing charges and $\mathrm{Ca2}+$ spikes of depolarization propagating throughout the compartment (reviewed by Waters et al., 2005). In this process, synaptic inputs are locally summated and temporo-spatially interact with each other via propagating $\mathrm{Ca} 2+$ spikes. Final integration will be determined at the interface between the input and output component of the neuron, typically the axon initial segment or axon 'hillock'. There, depolarization beyond a threshold set by the local concentration of voltage-gate channels will lead to the initiation of an action potential, an all-or-none $\mathrm{Na}^{+}$wave that will elicit a new round of synaptic transmission to neurons downstream in the network.

\subsubsection{The active zone of presynaptic release}

Axons are neuronal processes specialized in carrying action potentials to postsynaptic target neurons. Axon destinations may be meters away from the soma. Nonetheless, the propagation properties of action potentials ensure that transmitter release sites follow the activity pattern generated in the soma. These sites are found at axon terminals and at en route varicosities where presynaptic specializations assemble and release transmitter into the synaptic cleft in a regulated manner.

At the center of the presynaptic apparatus are the 'synaptic vesicles', specialized membrane-enclosed organelles which contain transporter proteins that actively load the vesicle lumen with transmitter molecules. The presynaptic machinery functions to fuse synaptic vesicles with the presynaptic membrane in a process of 
exceptionally rapid regulated secretion. Synaptic vesicles can be observed in electron micrographs to be 'docked' at specialized sites on the presynaptic membrane termed 'active zones', sites directly apposed to the postsynaptic membrane where fusion takes place.

At the active zone, proteins on the membrane of synaptic vesicles and on the presynaptic membrane form tight complexes which are primed to rapidly respond to the arrival of an action potential by eliciting membrane fusion. Intimately coupled to active zones are voltage-gated $\mathrm{Ca}^{2+}$ channels which are the first to respond to action potentials by increasing the $\mathrm{Ca}^{2+}$ conductance of the presynaptic membrane. Inflowing $\mathrm{Ca}^{2+}$ activates a component of the release-primed vesicles initiating a fusion event. Through this mechanism, action potentials trigger transmitter release.

A more refined description of the phenomenon of presynaptic release necessitates the consideration of many additional factors. Of the hundreds of synaptic vesicles typically observed at a release site, only a fraction is ready to undergo release at any given moment. From the population of such readily releasable transmitter vesicles, each one has a certain probability of undergoing fusion induced by a given action potential. These phenomena are governed by an array of molecules (reviewed by Wojcik \& Brose, 2007) which regulate the positioning of synaptic vesicles within a network of proteins collectively termed the 'cytomatrix and active zone' which characterizes release sites (reviewed by Rosenmund et al., 2003). The cytomatrix contains large proteins with multiple interaction modules that form a protein skeleton onto which synaptic vesicles are tethered. Vesicles are then selectively associated with active zones and are made fusion competent. The degree of association of these vesicles with $\mathrm{Ca}^{2+}$ channels and the state of the various fusion factors ultimately determines the probability of release and is a crucial node of regulation. Together with the transmitter content of the vesicle (referred to as 'quantal size'), regulation of release probability is a fundamental process that will determine the quantitative aspects of the signal transmitted to the postsynaptic cell. 


\subsubsection{The postsynaptic receptor apparatus}

The primary function of the machinery at the postsynaptic membrane of a fasttransmitting synapse is the translation of the chemical transmitter signal into a postsynaptic potential. The basis of this translation process lies in the molecular architecture of ligand-gated channels, or equivalently 'ionotropic receptors', the receptor components of electrical signaling at the postsynaptic membrane. Ionotropic receptors are membrane protein complexes composed of several constituent subunits. Each subunit spans the membrane several times, and multiple subunits conjoin to assemble a membrane complex with a gated hydrophilic pore. Following the general architecture of ion channels, pores are composed of bundles of membrane-spanning helices. The amphipathic nature of these helices allows the creation of a stable interface between the aliphatic environment of the lipid bilayer and the hydrophilic environment of the ion-conducting pore (reviewed by Zhorov \& Tikhonov, 2004).

As with other ion channels, the pore has open and closed states which are conformationally gated. This gating mechanism allows ionotropic receptors to respond to transmitter release from the presynaptic terminal by rapidly changing the conductive properties of the postsynaptic membrane. In the case of nicotinic acetycholine receptors, the gate consists of bulky side-chains on the pore-lining helices which sterically interfere with cation flux in the closed state (reviewed by Gay \& Yakel, 2007). The channel becomes conductive via a conformational shift of the helix bundles that unclog the pore. This conformational shift is induced via allosteric interactions caused by the binding of transmitter molecules to the extracellular surface of the channel complex.

In fast synaptic transmission, the time required from the arrival of an action potential in the presynaptic terminal to the eliciting of a postsynaptic potential is in the order of 1 millisecond. Such rapid processes require that all components involved in the mechanism are intimately linked. As $\mathrm{Ca}^{2+}$ channels at the active zone are coupled to docked vesicles, ionotropic receptors are positioned across from transmitter release sites, limiting the time necessary for transmitter molecules to diffuse from the release site to the receptors. Non-receptor components of the postsynaptic apparatus are specialized in ensuring just that; high receptor concen- 
trations in apposition to transmitter release sites. In electron micrographs a dense mesh of proteins has been observed lining the cytoplasmic side of the postsynaptic membrane. This structure is termed the 'postsynaptic density' (PSD) and is thought to correspond to a vast subsynaptic protein complex where receptor and signaling molecules are tethered on a protein scaffold (Palay et al., 1956; reviewed by Kennedy, 1993; Chen et al., 2008).

The proteins recruited to the PSD ultimately determine the characteristics of the postsynaptic response. While selectivity of receptor type is fundamental in determining postsynaptic potentials, synaptic responses are additionally tightly regulated by a convergence of cellular signals from the postsynaptic cell and signals from the extracellular space and other synapses. This makes synaptic transmission highly plastic, and the structural features of the PSD mediate the postsynaptic component of this plasticity. A variety of mechanisms of signaling and structural plasticity have been identified, including regulation of receptor integration, selection of subunit compositions of receptors (reviewed by Cull-Candy et al., 2001; Lüscher \& Keller, 2004; Greger et al., 2007), local post-translational modifications of receptors and PSD components, the recruitment of initiating molecules of biochemical signaling cascades (reviewed by Nguyen \& Woo, 2003; Colbran \& Brown, 2004) and through morphological determination of the postsynaptic membrane (reviewed by Alvares \& Sabatini, 2007).

\subsection{Synaptogenesis}

In cellular terms, synapses are specialized forms of adherence junctions as they are transcellular structures at which the plasma membranes of two cells are structurally linked. In this respect, the process of synapse formation, or 'synaptogenesis', implicates cell adhesion and intercellular signaling. However the unique functional properties of the chemical synapse make the formation of such specialized junctional complexes far more complicated than that of epithelial adherence junctions.

Selectivity and compatibility in pairing pre- to postsynaptic membranes are dominant features of synaptogenesis. Synaptic contacts are made between neurons 
whose somata can be a meter apart. For synaptogenesis to function, this entails mechanisms by which the projecting neuron crosses vast expanses of neural tissue without making synaptic contacts until it reaches its target neuron. Subsequent to target recognition, contact sites differentiate on both sides; the axon recruits a transmitter release apparatus and the receiving neuron is called to deploy postsynaptic components in precise apposition to the contact sites. As each neuron receives innervation of diverse transmitter systems, the postsynaptic cell must position a compatible postsynaptic apparatus at each site, i.e one with receptor types that are sensitive to the specific transmitter released. Once presynaptic and postsynaptic machineries are established, synapses are selected; many degenerate while others are selectively stabilized, going on to mature and evolve through processes of plasticity.

\subsubsection{The paradigm of neuromuscular junction formation}

Motor neuron axons exit the CNS and innervate muscle fibers making a unique form of synaptic contact termed the neuromuscular junction (NMJ). Compared to most central synapses, this cholinergic contact is giant and, being outside of the bustling mesh of the neuropile, it lends itself as an easily accessible model synapse. For these reasons the NMJ was the focus of the first studies of synaptogenesis. In the middle of the 1990s, seminal studies triumphantly provided a model which explained the synaptogenic processes at the NMJ. The 'Agrin hypothesis' quickly became textbook material, only to be revisited half a decade later as new evidence refuted some of its postulations, while critical elements in the model remained elusive. Nonetheless, the former studies and the Agrin hypothesis model have guided subsequent analyses of synapse formation and differentiation in the CNS.

The proposal of the Agrin hypothesis was based on a line of studies that began with experiments on an in vitro model of muscle innervation and were concluded with the study of the development of muscle innervation in mice with single gene deletions (reviewed by Daniels, 1997). Developing myotubes, the precursors of mature muscle fibers, express nicotinic acetylcholine receptors (AChRs) with a mostly diffuse distribution pattern on their surface. In mature muscle fibers, a sin- 
gle motor neuron terminal forms an extensive contact site on the fiber termed the 'motor endplate'. Upon postsynaptic differentiation, the endplate accumulates AChRs at a density of 10,000 receptor molecules per $\mu m^{2}$, in effect sequestering receptors that were distributed throughout the length of the sarcoplasmatic membrane. The Agrin hypothesis postulated that nerve-derived factors initiate signaling that leads to the spatial restriction and enrichment of AChR beneath contact sites in a manner independent from motor terminal transmission. The proteoglycan Agrin, secreted by incoming motor axon terminals and residing on the synaptic basal lamina between pre- and postsynaptic membranes, was shown to be critical for endplate formation (Gautam et al., 1996).

Agrin activates the muscle-specific receptor tyrosine kinase MuSK at contact sites on the sarcoplasmatic membrane (Glass et al., 1996), initiating signaling cascades with diverse effectors. These include cytoskeletal reorganization via the small Gprotein Cdc42, scaffold assembly with the PDZ-domain containing protein MAGI-1 and gene regulation via the MAP kinase pathway (reviewed by Strochlic et al., 2005). The crucial implication of MuSK in AChR clustering concerns the induction of AChR phosphorylation via Src family kinases through a pathway involving $\mathrm{Ra}$ psyn. Rapsyn binds to AChRs and is necessary for their phosphorylation and clustering (Guatam et al., 1995; reviewed by Sanes and Lichtman, 2001). Rapsyn is downstream of the Agrin-MuSK cascade and appears to serve as a scaffold, coupling AChRs with kinases to mediate their clustering.

The coherence of the schematic model of NMJ synaptogenesis is based on a chain of interactions in which Agrin activates MuSK through its associated receptor MASC, and the MuSK extracellular domain mediates cytosolic Rapsyn recruitment through membrane spanning RATL. However, both MASC and RATL are placeholder factors for unidentified cellular components, in effect they are conceptual figments necessary to reconcile the fact that Agrin has not been seen to associate with MuSK and that it is the extracellular domain of MuSK that is necessary to induce Rapsyn recruitment, Rapsyn being however a cytosolic protein. Additionally, an upset finding came when Agrin-deficient mice where interbred with mice lacking the synthetic enzyme for acetylcholine. In these mice, AChR clusters reappeared, indicating that Agrin may act to offset some dispersal effect of cholinergic trans- 
mission in the NMJ rather than functioning as the primary inducer of postsynaptic differentiation (Misgeld et al., 2005).

It becomes evident that in spite of intense focus on the study of NMJ synaptogenesis, many questions remain open. The paradigm of the Agrin hypothesis however, in spite of its serious shortcomings, set precedents that guided the study of synaptogenesis in the CNS. The key concepts in this model's legacy are the existence of synaptogenic factors derived from the presynaptic cell, the transsynaptic activation of signaling cascades and the resulting cytoskeletal rearrangements, the recruitment of scaffolds and the modification of receptors. These pathways lead to the molecular and morphological development of the postsynaptic apparatus, all carried out even in the absence of synaptic activity. Perhaps more importantly, the NMJ studies set a methodological precedent whereby single gene deletions resulted in the abolishment of postsynaptic differentiation; a phenomenon which has yet to be paralleled in the central synapse.

\subsubsection{Synaptogenesis in the central nervous system}

Although Agrin is expressed throughout the CNS, there is little evidence to suggest its implication in the formation of central synapses. Rather, it seems that a whole different set of protein systems is at work to elicit the guidance, formation and maturation of axodendritic synaptic contacts. In the CNS, synaptogenesis encompasses a sequence of discernible processes that appear to be mediated by distinct protein machineries.

Shortly after neuronal differentiation, neurites extending from the soma differentiate into axons and dendrites. Axons guided by their leading edge, the 'growth cone', extend, covering vast expanses of neuropile toward their target destinations. This first step, preceding synaptogenesis, is termed 'axon guidance' and entails the interaction of the growth cone with its surroundings through an assortment of repulsive and attractive signals. Key protein systems identified in mammals as being involved include Ephrins and Eph receptors, Netrins and Semaphorins (reviewed by Dickson, 2002). 
As these pathfinding cues guide the axonal growth cone in the vicinity of its destination, a set of extracellular signals prepare it for presynaptic differentiation. Molecules of the Wnt, fibroblast growth factor and Neurotrophin families derived from populations of target neurons (reviewed by Fox \& Umemori, 2006), and other molecules from glia cells in the target area (reviewed by Pfrieger \& Barres, 1997; Ullian et al., 2004) have been shown to promote aspects of contact formation and synaptic differentiation. Despite the potent effects of these molecules, it appears that they do not induce specific synapse formation. Rather, they act to promote the growth cone to a permissive state where synaptic differentiation is favorable, possibly spatially delineating a synaptogenic field.

As neuronal and dendritic processes prepare for synapse formation, filopodial extensions have been observed on both sides which make transient axodendritic contacts (Niell et al., 2004). Appearing as neurons sniffing each other out, these first manifestations of synaptogenesis are a process of target recognition. As with any cell-cell contact phenomenon, cell adhesion molecules play an important role, and prominent adhesion protein families such as Cadherins and Protocadherins have been identified at early axodendritic contacts (reviewed by Takeichi, 2007).

Often these initial contacts withdraw while others undergo synaptic differentiation, with the recruitment of protein components of the presynaptic and postsynaptic apparatus being recruited to contact sites. The process of synaptic differentiation is central to, indeed often synonymous with, synaptogenesis. During this process, crosstalk signaling between nascent presynaptic and postsynaptic membranes undoubtedly occur in order to coordinate the deployment of compatible release and receptor machineries in a spatially restricted manner.

As synaptic transmission itself inherently contains and relays the information of transmitter type, the most obvious mechanism to examine would be the involvement of synaptic release in synaptic differentiation. This hypothesis has, in broad terms, been concretely addressed. Synaptogenesis proceeded unhindered both in cultured neurons under chronic pharmacological activity blockade (Harms \& Craig, 2005) and in vivo in mice incapable of evoked or spontaneous synaptic release (Varoqueaux et al., 2002). It thus appears that synapse activation is dispensable for initial synaptic differentiation, and rather becomes critical in the later phases of 
synapse elimination and maturation. As in the NMJ, the focus has turned to adhesion and receptor mediated signaling as plausible mechanisms for synaptic differentiation.

Not surprisingly, the central synapse contains a wide array of adhesion molecules including members of typical cell adhesion protein families such as the Cadherin and immunoglobulin superfamilies. In a seminal study on the role of adhesion molecules in the process of synaptogenesis, Scheiffele et al. (2001) employed an in vitro system where neurons were co-cultured with non-neuronal cell-lines that heterologously expressed candidate adhesion proteins. In this co-culture assay, contact formation and synaptic differentiation were examined between axons and transfected cells. In this and subsequent studies employing similar approaches, several candidate adhesion proteins were screened (Table 1) leading to the identification of several adhesion systems that directly promote synaptic differentiation on both sides of the cleft.

Table 1: Adhesion molecules assayed for the induction of synaptic differentiation.

\begin{tabular}{|l|c|l|}
\hline N-Cadherin & - & Scheiffele et al. 2000 \\
L1 & - & Scheiffele et al. 2000 \\
TAG-1 & - & Scheiffele et al. 2000 \\
Agrin & - & Scheiffele et al. 2000 \\
EphrinB1 & - & Scheiffele et al. 2000 \\
Neuroligin 1 & + & Scheiffele et al. 2000 \\
Neuroligin 2 & + & Scheiffele et al. 2000 \\
SynCAM1 & + & Biederer et al. 2002 \\
NgCAM & - & Graf et al. 2004 \\
Neurexin 1 $\beta$ & + & Graf et al. 2004 \\
NCAM-140 & - & Sara et al. 20005 \\
SALM2 & $(+)$ & Ko et al. 2006 \\
Netrin-G ligand & + & Kim et al 2006 \\
Neurexin 1a & + & Kang et al. 2007 \\
\hline
\end{tabular}


The Neuroligin-Neurexin heterophilic adhesion system was the first and best characterized complex shown to be involved in the induction of synaptic differentiation. Postsynaptic Neuroligins are clustered with presynaptic Neurexins and each mediates the recruitment of the respective synaptic machineries. Studies of deletion mutant mice have shown that synapses do not function properly in the absence of Neuroligins (Varoqueaux et al., 2006) or a-Neurexins (Missler et al., 2003), possibly due to incomplete recruitment of components of the pre- and postsynaptic apparatus. In the case of a-Neurexin deletion, the number of inhibitory synapses formed in the brain is additionally reduced twofold.

Other adhesion proteins have been similarly reported to induce synaptic differentiation such as SynCAM1, a homophilic adhesion system (Biederer et al., 2002). A postsynaptic protein, SALM2, whose presynaptic ligand has not been identified, was shown to recruit excitatory postsynaptic scaffolds. Similarly, Netrin-G ligands (Ko et al., 2006), postsynaptic glycosyphosphatidylinositol anchored proteins binding to Netrin-G induce excitatory presynaptic differentiation (Kim et al., 2006). It thus appears that synaptic differentiation in the CNS can be mediated by several adhesion systems which recruit the components of the pre- and postsynaptic machineries at sites of contact.

While the different adhesion systems involved in synapse differentiation exhibit overlap and redundancy of functions, their potential for mediating synapse specificity is currently under scrutiny. In contrast to the NMJ, where synaptogenesis involves a single transmitter system, indeed a single synapse, at central synapses synaptogenesis is carried out in parallel at thousands of positions on a given neuron and involves an assortment of at least six ionotropic transmission types; glutamatergic, GABAergic, glycinergic, cholinergic serotonergic and purinergic. Specificities pertaining to transmitter systems have been observed with SynCAMs, SALMs and Netrin-G ligands functioning exclusively at glutamatergic synapses, while Neuroligins-Neurexins function potentially in all of the first four transmitter systems -with the last two yet to be examined- in an isoform specific manner (see $\S 1.4 .3)$.

As critical as synaptogenesis is in the development of neuronal networks, so are subsequent processes which prune many contacts and selectively stabilize others. 
These processes may, to some extent, involve molecules implicated in synaptogenesis like Neuroligin1 (Chubykin et al., 2007), but are fundamentally different in nature as synaptic activity appears to be a major criterion for network maturation.

\subsection{Synaptic inhibition}

Excitatory and inhibitory synapses function in concert to shape network activity patterns in neuronal ensembles. Excitatory inputs give rise to $\mathrm{Ca} 2+$ spikes which in turn are shunted when propagating through sites of inhibitory input. The importance of synaptic inhibition has been elegantly demonstrated in the hippocampus where field potential oscillations exhibit stereotyped wave patterns. The discrete elements of these patterns were shown to arise through the activity of distinct inhibitory interneuron populations contacting hippocampal pyramidal neurons, ultimately giving field potential oscillations their characteristic shapes (reviewed by Bartos et al., 2007).

Fast synaptic inhibition in the CNS is mediated through the transmitter systems GABA and glycine. The two types of inhibition predominate in different regions of the CNS with glycinergic transmission being most prominent in brainstem centers and the spinal cord, and GABAergic transmission carrying out the bulk of inhibition in the forebrain. Additionally, the two inhibitory transmitter systems have different kinetics in their inhibitory postsynaptic responses. In motor neurons glycine mediates fast decaying inhibitory postsynaptic potentials while GABA-mediated inhibition decays slower (Jonas et al., 1998). The opposite appears to be the case in the cerebellum (Dumoulin et al., 2001).

Despite such differences, both transmitter systems share many common components including the same vesicular transporter (Wojcik et al., 2006), the same postsynaptic scaffolding protein (see $\S 1.4 .2$ ) and receptor subunits of the same family (Xue, 1998). Evidence pointing to GABA and glycine co-releasing terminals opposed to mixed postsynaptic sites, containing both GABA and glycine receptors (GlyRs), has been accumulating (Jonas et al., 1998; Dumoulin et al., 2001). The variety that arises from the different combinations of transmitter and the variability 
in receptor subclasses gives synaptic inhibition the versatility to tailor neuron spiking patterns to the needs of each CNS circuit.

\subsubsection{Glycine receptors}

Transmission based on glycine is traditionally associated with circuits mediating reciprocal and recurrent inhibition in the spinal cord. Poisoning by strychnine, a specific blocker of GlyRs, results in muscle stiffness and convulsions due to motor neuron hyperexcitation and concurrent activation of antagonistic muscles. The specificity of strychnine for glycinergic transmission made it an invaluable tool for the analysis of the glycinergic synapse. Employing strychnine in affinity purification methods led to the isolation of the GlyR and to the cloning of its genes (Pfeiffer et al., 1982). Radiolabeled strychnine was instrumental in the first characterization of GlyR distribution patterns in the CNS. Though most prominent in spinal cord circuits, glycinergic transmission can be found in other regions of the CNS -the brainstem, retina and auditory circuits being the most noted instances. Low levels of GlyRs have additionally been observed in the cerebellum and at diffuse sites in the hippocampus (Fujita et al., 1991).

GlyRs are anion selective ionotropic receptors belonging to the pentameric ligandgated ion channel (LGIC) family which includes nicotinic acetylcholine, serotonin type 3, GABAA and GABAc receptors. Each GlyR molecule is most likely composed of a mix of five membrane spanning proteins of two classes; $a$, with four possible subunits, and $\beta$ with a single subunit. The probable stoichiometry of GlyRs is $3 a 2 \beta$, with Glycine binding to a pocket formed at the interface between a and $\beta$ subunits to induce the opening of the $\mathrm{Cl}^{-}$channel pore (reviewed by Lynch, 2004). On the cytoplasmic face of the postsynaptic membrane, the GlyR $\beta$ subunit extends a loop which binds to the scaffolding protein Gephyrin (Meyer et al., 1995) which co-purifies with strychnine-bound GlyR preparations (Langosch et al., 1992) and through which it remains clustered at postsynaptic sites (Kirsch et al., 1993; Feng et al., 1998). 


\subsubsection{GABA receptors}

GABA was the first compound to be identified as a neurotransmitter mediating synaptic inhibition (Krnjevic \& Schwartz, 1966); the devoted transmitter of around $17 \%$ of synapses in the mammalian brain. A striking feature of GABA-mediated inhibition is the heterogeniety of transmission arising from a panoply of receptor subtypes. Like GlyRs, GABA receptors are ligand-gated chloride channels of the LGIC family. They have been the focus of intense study due to the fact that they represent target sites for major thumotropic substances like barbiturates, benzodiazepines and alcohol (reviewed by DeFeudis, 1983).

The major group of ionotropic GABA receptors, $G A B A_{A}$ receptors $\left(G A B A_{A} R\right)$, are pentamers composed of varying combinations of different classes of subunits; the a class contains six possible subunits, the $\beta$ and $\gamma$ classes three, while subunit classes of $\delta, \varepsilon, \theta$ and $\pi$ are solitary. The contribution of each class of subunits to the pentamer is unknown, though studies point to a prevalent stoichiometry of $2 a$ : $2 \beta: 1 \gamma$ (reviewed by Sieghart et al., 1999). The precise compositions of $G A B A_{A} R$ molecules have been shown to be region-dependent and, more strikingly, dependent on the cell-type they are innervated by. A typical example of the latter can be observed in the interneuron innervation of the hippocampus. GABAergic contacts from CCK-positive basket cells onto the somata of pyramidal neurons lead to the postsynaptic clustering of a2-containing receptors (Nyiri et al., 2001), while GABAergic innervation onto the somata from parvalbumin-positive basket cells is received by a1-containing receptors (Fritschy \& Möhler, 1995). Examples of region-specific subunits include a6, exclusively expressed in the cerebellum, and a5, predominantly expressed in the hippocampus.

The complex distribution pattern of $\mathrm{GABA}_{A} \mathrm{R}$ subunits is thought to accommodate the characteristics of inhibition to the needs of the circuitry. Heterologous expression studies in Xenopus laevis oocytes have shown that the subunit composition of $\mathrm{GABA}_{A} \mathrm{Rs}$ principally determines channel gating, kinetics and pharmacological characteristics (reviewed by Lüddens \& Wisden, 1991). Mouse genetic studies involving the ablation of single $\mathrm{GABA}_{A} \mathrm{R}$ subunit genes have been confounded by complex compensatory phenomena, however, several striking functions of the different subunits in vivo were revealed (reviewed by Rudolph \& Möhler, 2004). Not 
surprisingly, the most severe phenotype from the $G A B A_{A} R$ subunit knockout mice came from the deletion of $\gamma 2$, the most abundant and ubiquitous synaptic $G A B A_{A} R$ subunit. Y2 deletion-mutant mice exhibited perinatal lethality while benzodiazepine binding sites where diminished. Knockout of the $\beta 3$ gene also resulted in perinatal lethality with a small minority of pups surviving to adulthood and exhibiting greatly attenuated phenotypic deficits. Perhaps more intriguing is the absence of gross deficits of mice lacking a1 or $\beta 2$, the most abundant subunits of their respective classes. Though a1-deficient mice lost more than half of total $\mathrm{GABA}_{A} \mathrm{Rs}$ in the brain, the only grossly observable phenotype these mice exhibited was tremor when handled (Sur et al., 2001).

\subsubsection{Tonic and phasic inhibition}

The first localization studies of $\mathrm{GABA}_{\mathrm{A}} \mathrm{Rs}$ by immunolabeling surprisingly showed them not to correlate with synaptic terminals releasing GABA, but to exhibit a more diffuse distribution pattern. The development of unconventional fixation techniques later permitted the observation of the punctate staining anticipated for neurotransmitter receptors (Fritschy et al., 1998). Since then, it has become clear that there are two major fractions of GABA receptors; synaptic receptors clustering opposite sites of transmitter release, and diffuse extrasynaptic receptors. Importantly, both fulfill distinct physiological roles.

Electrophysiologically, inhibition appears in two distinct forms termed 'tonic' and 'phasic'. Tonic inhibition sets a baseline state of inhibition, effectively regulating regional activation thresholds of neuronal populations. This steady-state inhibition can be seen as a depolarizing shift in the resting potential of neurons devoid of synaptic activity upon application of $\mathrm{GABA}_{A} \mathrm{R}$ blockers. This indicates that, independently from synaptic transmission, GABA-mediated inhibition is exerted on neurons at a steady state. The pool of diffuse extrasynaptic $\mathrm{GABA}_{A} \mathrm{Rs}$ has a postulated function in tonic inhibition as they can respond to low ambient concentrations of GABA. The importance of extrasynaptic inhibition is exemplified by the correlation of epileptogenic activity with the breakdown of tonic inhibition and by its increasingly recognized roles in modulating physiological network activity (reviewed by Semyanov et al., 2004). 
In contrast to tonic inhibition, phasic inhibition is observed to correlate with synaptic activity. This form of inhibition originates from the firing patterns of GABAergic interneurons and is mediated by synaptically clustered GABA $\mathrm{As}$ (Brickley at al. 1999). The difference between receptors involved in tonic and phasic inhibition again appears to lie in their subunit composition. Detailed morphological analysis of $\mathrm{GABA}_{A} \mathrm{R}$ subunit distribution patterns has shown that the $\delta$ and a 6 subunits are found in extrasynaptic receptor pools, while the $\mathrm{Y} 2$ subunit associates with all synaptically localized receptor clusters. With these subunits representing the polar ends of the spectrum of synaptic versus extrasynaptic localization, other subunits are varyingly distributed, with a1 and a2 tending toward the synaptic and $a 4$ and a5 toward the extrasynaptic receptor pools (reviewed by Lüscher \& Keller, 2004).

A mechanistic link between receptor localization and subunit composition became apparent when cultured neurons from $\mathrm{y}^{2}$-deficient mice were observed to be devoid of synaptic clusters of $\mathrm{GABA}_{\mathrm{A}} \mathrm{Rs}$ (Essrich et al., 1999). While it remains unclear how this subunit mediates clustering, one subsequent study has identified transmembrane domain 4 of the $\mathrm{Y} 2$ subunit as being necessary for this process (Alldred et al., 2005). Other $y$ subunits, which all have highly conserved intracellular loops, can rescue the clustering deficit phenotype of neurons lacking Y2 (Baer et al., 1999; Baer et al., 2000) suggesting a possible role of cytosolic sequences as well. Conversely, mice lacking $\mathrm{GABA}_{\mathrm{A}} \mathrm{R}$ subunit a5 show a selective decrease in extrasynaptic receptors (Fritschy et al., 1998), while a5-containing receptors

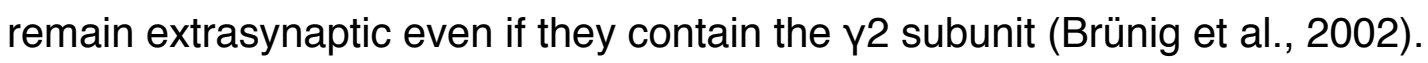

Other subunits with potential roles in the differential localization of GABAARs include the three $\beta$ subunits which appear to determine polar sorting. In polar epithelial cells exogenously expressed $\beta 1$-containing receptors show a non-polar distribution. $\beta 2$-containing receptors, however, traffic directly to the basolateral membrane, which is considered analogous to the somatodendritic compartment in neurons. In contrast, $\beta 3$-containing receptors are first targeted to the apical membrane -analogous to the axonal compartment in neurons- and subsequently to the basolateral membrane. It thus appears that in neurons, in addition to modulating $\mathrm{GABA}_{A} \mathrm{R}$ channel properties, the different subunits may also participate in cellular mechanisms that determine their participation in tonic versus phasic inhibition and in synapse type-specific targeting. 


\subsubsection{Inhibitory receptor clustering}

Despite the information accumulating on the cellular properties of different $\mathrm{GABA}_{A} \mathrm{R}$ subtypes, the key mechanisms of their clustering to postsynaptic sites remain elusive. In contrast, GlyR synaptic targeting appears to be mediated by a rather straightforward mechanism involving direct subsynaptic scaffolding of receptor molecules. The scaffolding protein Gephyrin is tightly associated with synaptic GlyR as exemplified by its identification as a co-purifying component of GlyR preparations (Langosch et al., 1992). Gephyrin is omnipresent at glycinergic postsynaptic sites and is required for the formation of GlyR postsynaptic clusters which do not form in its absence (Kirsch et al., 1993; Feng et al., 1998). As mentioned previously, GlyR pentamers are normally composed of $a$ and $\beta$ subunits. Given that there is only one form of GlyR $\beta$ subunit, this makes all pentamers have a constant component. The cytosolic loop of the omnipresent GlyR $\beta$ subunit has a motif that directly binds to Gephyrin (Meyer et al., 1996). Postsynaptically clustered Gephyrin appears to capture laterally diffusing GlyR at synaptic sites as seen in single-particle tracking studies (Meier et al., 2001), leading to receptor clustering. It thus appears that GlyR dynamics appear to take place largely at the plane of the plasma membrane, between synaptic and extrasynaptic pools.

The presence of Gephyrin at GABAergic synapses (Craig et al., 1996) brought up the appealing notion that $\mathrm{GABA}_{\mathrm{A}} \mathrm{Rs}$ may similarly be scaffolded to the postsynaptic membrane. This was reinforced by the observation that synaptic $G A B A_{A} R$ clusters were absent from mice lacking Gephyrin (Kneussel et al., 1999; Essrich et al., 1999). However, the parallels with GlyR clustering stopped there as a series of subsequent studies dampened the excitement about the possible universality of the model. Indeed, synaptic $\mathrm{GABA}_{A} \mathrm{R}$ persisted to some extent in Gephyrin knockout neurons (Lévi et al., 2004), while subsets of GABAergic synapses are physiologically devoid of Gephyrin in vivo (Sassoé-Pognetto et al., 2000). Additionally, $\mathrm{GABA}_{A} \mathrm{R}$ subunit knockout mice lacking synaptic receptors are also devoid of postsynaptic Gephyrin clusters (Essrich et al., 1999; Kralic et al., 2006) even when receptor deletion is induced after synapse maturation (Schweizer et al., 2003). This raises the bewildering possibility that receptors may recruit Gephyrin to the postsynaptic membrane rather than the other way around. Perhaps the most con- 
founding evidence against a scaffolding model of $\mathrm{GABA}_{A} \mathrm{R}$ clustering is the conspicuous lack of data pointing to an interaction between $\mathrm{GABA}_{\mathrm{A}} \mathrm{Rs}$ and the Gephyrin scaffold -although a weak association of the a2 subunit with Gephyrin was recently reported (Tretter et al., 2008).

Other approaches to address the question of $\mathrm{GABA}_{A} \mathrm{R}$ synaptic clustering similarly fall short of giving coherent answers. Presynaptic innervation is dispensable for receptor clustering (Brünig et al., 2002), while, yet again, receptor clustering seems to promote presynaptic innervation ( $\mathrm{Li}$ et al., 2005). Other components of the inhibitory postsynapse, like the Dystrophin glycocomplex (Kneussel et al., 1999; Lévi et al., 2002; Grady et al., 2006) and Neuroligin 2 (Schema 1, see $\S$ 1.3.4 and chapter 4), have been shown to effect the synaptic targeting $G A B A_{A} R s$, however their functional roles remain largely elusive. It is thus safe to say, that despite intense efforts to provide a coherent model for the clustering of $G A B A_{A} R s$

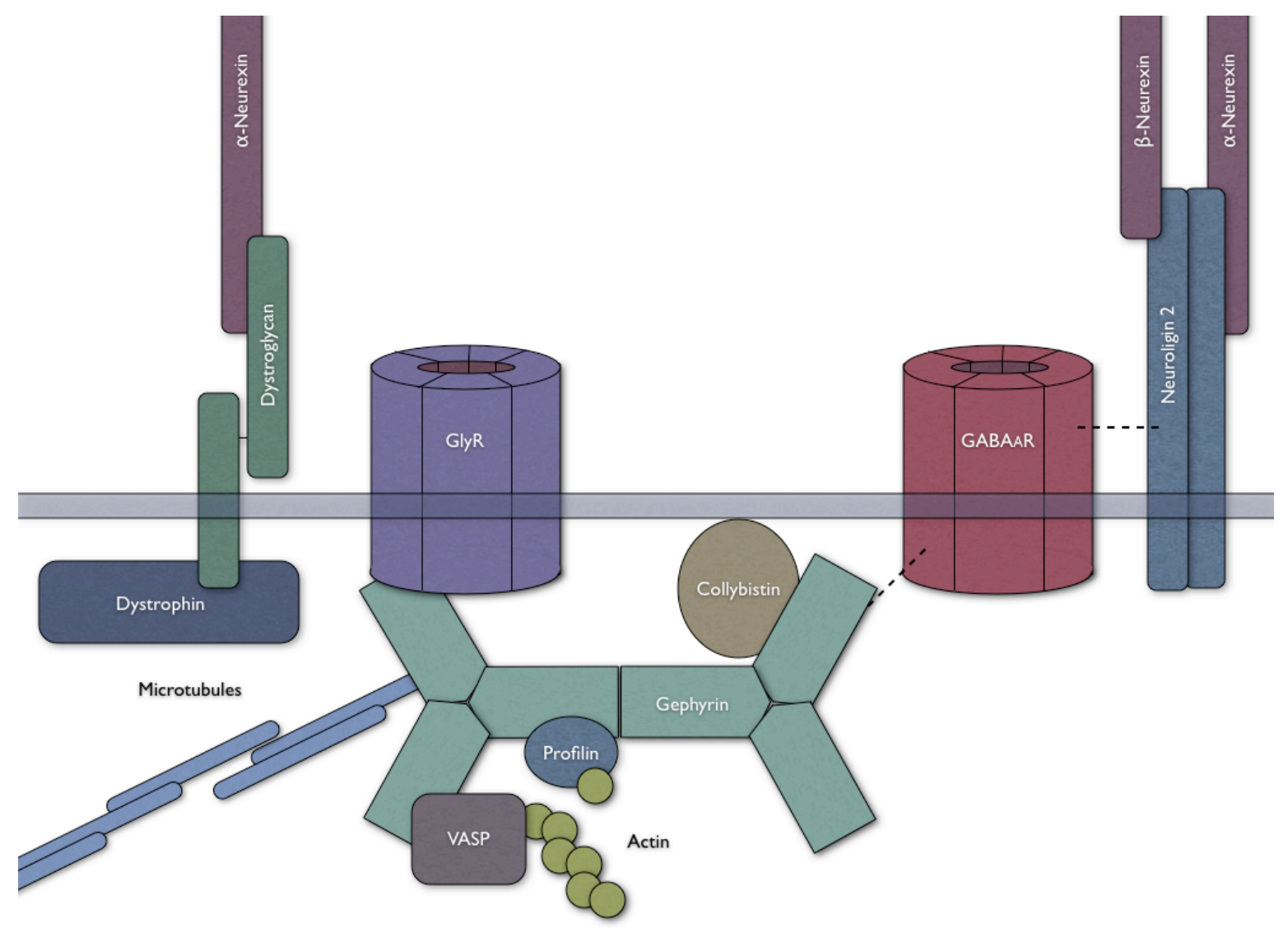

Schema 1: Representation of the inhibitory postsynapse. Glycine and/or GABAA receptors accumulate at the inhibitory postsynaptic membrane. Receptors are directly or indirectly linked to Gephyrin which lines the cytoplasmic side of the postsynaptic membrane in hexamer-based lattices. Collybistin binds to Gephyrin and promotes its association with the postsynaptic membrane, while Profilin, VASP and microtubule-associated proteins link to the cytoskeleton. The adhesion molecules Neuroligin 2 and Dystroglycan are also specifically clustered at inhibitory postsynaptic membranes, the latter being associated to cytoplasmic proteins of the Dystrophin family. 
mediating phasic inhibition at postsynaptic sites, current evidence has only uncovered bits and pieces of the physiological mechanisms at work.

\subsection{Assembly mechanisms of the postsynaptic apparatus}

The postsynaptic apparatus is an extensive and intricate complex of proteins formed through an elaborate network of interactions at the postsynaptic membrane and juxtamembranal space (see $\S 1.1 .3$ ). In electron micrographs this expansive complex can be seen as an electron-dense thickening of the plasma membrane termed the 'postsynaptic density' (PSD). The prototypical PSD is found at glutamatergic synapses, however, inhibitory synapses also show postsynaptic electrondense material, albeit less pronounced.

Extensive characterization of the protein components of the excitatory PSD has been facilitated by its biochemical features. Treatment of synaptic preparations with non-ionic detergent extracts non-synaptic membranes and the presynaptic component, leaving the PSD intact. On the basis of this property, biochemical purification methods were established (Cotman et al., 1974) that have allowed detailed qualitative, quantitative and structural characterization of the PSD employing proteomic and electron microscopy techniques (reviewed by Sheng et al., 2007). These analyses have implicated hundreds of different proteins with diverse functions, covering diverse receptor classes, adaptor proteins, cell adhesion proteins, cytoskeletal elements and regulators, signaling and trafficking proteins as well as components of the translational machinery and metabolic enzymes. The wealth and diversity of the PSD proteome demonstrates the complexity of the excitatory postsynaptic apparatus.

Despite the multiplicity and intertwined nature of interactions at the PSD, quantitative proteomic readouts along with domain structure information on PSD components have allowed the postulation of an organizational framework of PSD structure that becomes invaluable to studies of postsynaptic assembly mechanisms. A hefty number of proteins in the PSD are large cytosolic molecules that contain multiple protein interaction modules and have multimerization capacities. This do- 
main architecture makes them suited to serve as a structural core of multiprotein complexes. Connected to adhesion protein and lipid components of the postsynaptic membrane, they can serve as scaffolds onto which proteins with specific functional tasks of the postsynaptic apparatus attach.

\subsubsection{PDZ domain scaffolds}

Considering that the primary role of the postsynapse is to relay the chemical signal from the presynaptic cell and translate it into a membrane potential change, the primary question in the study of its assembly is how ionotropic receptors are integrated in the postsynaptic apparatus. In the excitatory PSD, one protein interaction module appears at the center of this process. This module, termed 'PDZ' domain after the prototypic proteins which harbor it (Postsynaptic density-95, Disks large and Zonula occludens-1), is present in multiple copies in numerous scaffolding proteins abundant at the PSD. Prominent synaptic proteins containing PDZ domains belong to the MAGuK protein family, with PSD-95 as its prototypic member, and the MAGI family with S-SCAM as its prototypic member.

Stoichiometric evaluation of the PSD has shown that PSD-95 is its most abundant scaffolding protein, contributing an average of 300 molecules to one postsynapse (Chen et al., 2005). The three tandem PDZ domains it contains recognize short, typically C-terminal, peptide sequences with affinity constants varying from hundreds of micromolar to the sub-micromolar range (Saro et al., 2007). Such 'PDZbinding motifs' are present in the cytosolic tails of many transmembrane proteins resident at the postsynapse, including subunits of acetylcholine and all classes of ionotropic glutamate receptors, adrenergic and glutamatergic metabotropic receptors, receptor tyrosine kinases, voltage-gated ion channels and adhesion proteins of the Neuroligin family (reviewed by Kim \& Sheng 2004).

Studies employing electron tomography localize PSD-95 to the most membraneproximal reaches of the PSD (Chen et al., 2008). It is additionally reported to associate with the lipid phase of the postsynaptic membrane itself via posttranslational acylation (Craven et al., 1999). Through its positioning, PSD-95 can interact with membrane bound PDZ ligands, as well as with deeper components of the postsynaptic apparatus. These notions provide a schematized view where 
PSD-95 and related scaffolding proteins create the interface between the integral membrane component of the PSD and its proteinacious subsynaptic component.

The direct interaction of PSD-95 and related proteins with the postsynaptic adhesion molecules of the Neuroligin family (Irie et al., 1997; Meyer et al., 2004) is considered especially important in bringing the PSD in register with the presynaptic release machinery. Neuroligins bind presynaptic Neurexins, thus forming a protein bridge that spans the synaptic cleft. On both ends of this junction are PDZ-binding motifs which attach the adhesion complex to pre- and postsynaptic scaffolds. This transsynaptic link can align PSD-95 accumulations with transmitter releasing terminals. As PSD-95 and related proteins feature at the core of PSD architecture, they appear capable of mediating the assembly of the postsynaptic apparatus (Elias et al., 2006) in apposition to presynaptic contacts. Equivalent PDZ-mediated assemblies have been described on the presynaptic side in the deployment of the cytomatrix and active zone.

In support of the above hypothesis is the capacity of Neuroligin and Neurexin clustering to induce postsynaptic and presynaptic differentiation in vitro. However, mouse genetic studies involving PSD-95 (Migaud et al., 1998), Neuroligins (Varoqueaux et al., 2006) and Neurexins (Missler et al., 2003) have demonstrated that such a mechanism is one in a series of parallel functioning mechanisms. The builtin redundancy of the PSD, in contrast to the motor end plate, make readouts of single component deletion difficult to interpret. They do however go a long way in addressing the functional subtleties that one would expect in a system of such complexity.

\subsubsection{The Gephyrin scaffold}

Inhibitory synapses not only contain distinct postsynaptic receptors to respond to inhibitory transmitters; the postsynaptic apparatus accompanying them is composed of an entirely different set of proteins. In stark contrast to the elaborate models describing the glutamatergic postsynapse, current models of the inhibitory postsynapse contain only a handful of molecules (Schema 1). This meager compilation of synaptic components is unlikely to reflect the simplicity of the inhibitory postsynapse. Rather it can be attributed to the biochemical properties of glyciner- 
gic and GABAergic postsynapses, which have thus far not led to a preparation of a purified fraction equivalent to the PSD of glutamatergic postsynapses.

Despite our limited knowledge on the constituents of inhibitory postsynapses, a few functionally critical components have been documented, most notable of which are its scaffolding components. There is a conspicuous lack of PDZ domain proteins at inhibitory postsynapses, with two recently identified exceptions possibly being isoforms of GRIP1 (Charych et al., 2004) and S-SCAM (Sumita et al., 2006). Instead, the inhibitory postsynaptic apparatus is dominated by a single scaffolding protein, Gephyrin. The evidence pointing to Gephyrin as having a scaffolding role at inhibitory synapses (see $\S 1.3 .3$ ) is indeed far more convincing than for any putative excitatory scaffold. The genetic evidence clearly points to the necessity of Gephyrin for synaptic clustering of the vast majority of inhibitory synapses, and in the case of glycinergic postsynapses, the proposed models are coherent with complementing functional and structural evidence. Though many more proteins will likely prove to be significant in the inhibitory postsynapse, Gephyrin is undoubtedly central to the mechanisms that dictate inhibitory postsynapse assembly.

Gephyrin is the product of a single gene that has no close paralogues. Orthologous comparison testifies that Gephyrin, in terms of synaptic proteins, is a "living fossil" of sorts, having homologues in bacteria and plants. A synaptic protein in prokaryotes is a curious notion, plausible only in light of the pleotropic function of Gephyrin both as a synaptic scaffold and as a metabolic enzyme (Feng et al., 1998). The ancestral function of Gephyrin is the synthesis of MoCo, a cofactor necessary for the function of molybdoenzymes. In mammals, Gephyrin is ubiquitously expressed serving both enzymatic and synaptic functions.

Gephyrin has no identified modular domains and its structure is broadly described in terms of two Escherichia coli genes, MoeA and MogA, which encode serial enzymes in the MoCo biosynthetic pathway. These two sequences appear to have fused into one gene, presumably enhancing turnover of the pathway, giving rise to eukaryotic Gephyrin harboring an N-terminal MogA sequence, termed G-domain, and a C-terminal MoeA sequence, termed E-domain (Schema 6). In between the Gephyrin $G$ and $E$ domains is a stretch of approximately 150 residues termed the 'linker region', despite lack of evidence of it being unstructured. Consistent with its 
role as a scaffolding protein, Gephyrin is assumed to form multimers through Gdomain-mediated trimerization and E-domain-mediated dimerization, which would allow it to be assembled into hexagonal lattices (Saiyed et al., 2006).

There have been several proteins shown to interact with Gephyrin (Schema 6). The most prominent and best documented interaction partner of Gephyrin is the $\beta$ subunit of the GlyR. An 18-residue sequence within the large cytoplasmic loop of the $\beta$ subunit binds to the Gephyrin E-domain comprising a Gephyrin-binding site sufficient to mediate recruitment to Gephyrin structures in cell-lines (Meyer et al., 1996). This interaction is essential for Gephyrin to function as a scaffold for GlyRs.

An equivalent interaction with $\mathrm{GABA} A \mathrm{R}$ was long sought after but remains largely elusive. A direct interaction of Gephyrin with $\mathrm{GABA}_{\mathrm{A}} \mathrm{Rs}$ has recently been suggested. Tretter et al. (2008) demonstrated a hydrophobic interaction of the $\mathrm{GABA}_{A} \mathrm{R}$ a2 subunit cytoplasmic loop with Gephyrin. However, the weak nature of the interaction and its specificity to the a2 subunit and to a subset of inhibitory synapses makes it an unlikely candidate to act as a central link mediating Gephyrin scaffolding at GABAergic synapses. A protein named GABARAP, interacting with both Gephyrin (Kneussel et al., 2000) and GABA ${ }_{A}$ s (Wang et al., 1999), was proposed to serve as an adaptor protein for the GABAergic postsynaptic scaffold, indirectly linking receptors to Gephyrin. GABARAP however is not present at synaptic sites and most likely is involved in processes of the secretory pathway (reviewed by Coyle et al., 2004).

While several aspects of Gephyrin function remain largely unelucidated, several studies clearly point to the association of Gephyrin with cytoskeletal elements. Very soon after Gephyrin was identified as a component of GlyR preparations, it was characterized as a microtubule-associated protein. It remains unclear how Gephyrin interacts with microtubules, whether it is directly or indirectly via the Dynein light chain, a microtubule motor protein and Gephyrin interactor. Upon overexpression in non-neuronal cells, Gephyrin forms large aggregate structures, termed 'blobs' (Kirsch et al., 1995), which appear to be nucleated around microtubule-organizing centers (Maas et al., 2006). A link of Gephyrin with the actin cytoskeleton has also been proposed as it has been seen to associate with actin structures and interacts with the actin-binding proteins Profilin and VASP 


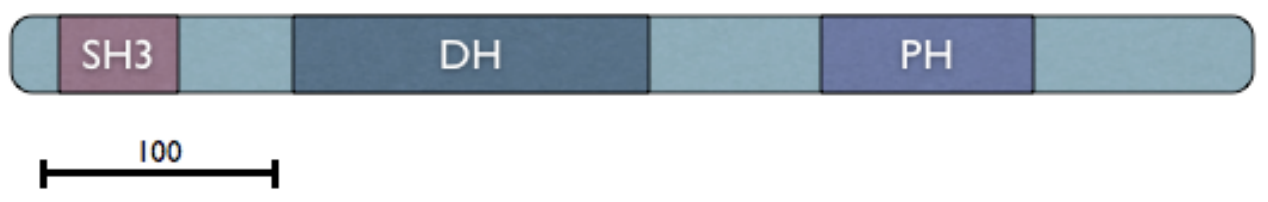

Schema 2: Collybistin domain structure. Collybistin (520 residues) contains three modular domains: SH3 (residues 20-71), DH (residues 118-267) and PH (residues 339-428). Residue numbering is given for rat Collybistin variant $\mathrm{CB} 1_{\mathrm{SH}_{3+}}$. Scale bar represents amino acid residues.

(Mammoto et al., 1998; Giesemann et al., 2003; Bausen et al., 2005). The synaptic relevance of the association of Gephyrin to the cytoskeleton is unclear, however, its interaction with Dynein light chain and association with the microtubule network is reportedly involved in its transport in complex with GlyRs to dendritic postsynaptic sites (Maas et al., 2006).

Gephyrin is a cytosolic protein that tends to form cytoplasmic aggregates in nonneuronal cells. Given its strict association with the postsynatic membrane in neurons, Gephyrin is thought to require a neuron-specific mechanism to be targeted to the plasma membrane. A neuronal protein, Collybistin, was identified as a component of this hypothetical mechanism through a yeast-two-hybrid screen for Gephyrin interacting proteins (Kins et al., 2000). A minor splice variant of Collybistin was shown to induce reorganization of the subcellular distribution of Gephyrin from cytoplasmic to plasma membrane-associated when the two proteins were coexpressed in non-neuronal cells. A Collybistin mutation found in a patient with hyperekplexia (Harvey et al., 2004) and Collybistin deletion in mice document the importance of Collybistin for the targeting of Gephyrin to the postsynaptic membrane in vivo, though region-specific compensatory mechanisms appear to function in parallel (Papadopoulos et al., 2007).

Collybistin, a homologue of the Dbl protein family of GDP/GTP exchange factors, has a domain organization that consists of a N-terminal SH3 domain, a central Dbl Homology $(\mathrm{DH})$ domain and a C-terminal Pleckstrin Homology $(\mathrm{PH})$ domain (Schema 2). The DH domain has been reported to interact with the Gephyrin $\mathrm{E}$ domain, leading to a stable complex of Gephyrin-Collybistin both at cytoplasmic and membrane-associated structures. The $\mathrm{PH}$ domain, a putative membrane phosphoinositide-binding module, was shown to be critical, along with the Gephyrin-binding domain, for the translocation of Gephyrin to the plasma mem- 
brane. Conversely, the SH3 domain acts as an inhibitor of the membrane-targeting function of Collybistin. Thus, active Collybistin contains the $\mathrm{DH}$ and $\mathrm{PH}$ domains but lack the SH3 domain (Harvey et al., 2004). Collybistin mRNA variants where the SH3 domain was spliced out represent less than $5 \%$ of Collybistin transcripts in vivo, while SH3-lacking Collybistin is not detectable at the protein level in the brain (Kins et al., 2000; Harvey et al., 2004; Papadopoulos et al., 2007). As active Collybistin lacking the inhibitory $\mathrm{SH} 3$ domain does not appear to have a major physiological role, the existence of Collybistin activity regulators that reverse the inhibitory action of the $\mathrm{SH} 3$ domain in neurons is implied.

Despite the strong evidence for Gephyrin functioning as a synaptic scaffolding molecule at glycinergic and GABAergic postsynapses, the mechanisms involved in the synaptic positioning of the Gephyrin scaffold have been addressed only to a limited extent. Pharmacological data implicated glycinergic transmission as being necessary for the postsynaptic clustering of receptors; treatment with strychnine and L-type voltage-gated $\mathrm{Ca}^{2+}$ channel blockers both disrupt postsynaptic receptor clustering. This indicates that at developing glycinergic synapses, where transmission is depolarizing due to high intracellular $\mathrm{Cl}^{-}$concentrations, an activitydependent mechanism may function to demarcate sites of postsynaptic differentiation (reviewed by Kneussel \& Betz, 2000). However, subsequent evidence confirming this hypothesis has not been forthcoming, while synaptic activation appears to be dispensable for early GABAergic postsynaptic differentiation (Varoqueaux et al., 2002; Wojcik et al., 2006). Though assembly of the inhibitory postsynaptic apparatus relies on the Gephyrin scaffold, what induces the deployment of the scaffold at nascent postsynaptic sites remains unknown.

\subsubsection{The Neuroligin family of adhesion proteins}

Adhesion molecules are crucial components in the assembly of junctional complexes. At the synaptic junction, the Neuroligin-Neurexin transsynaptic adhesion complex has a demonstrated role in the assembly of both pre- and postsynaptic specializations. Neurexins appear to function as receptors for Neuroligin induced presynaptic differentiation, while Neuroligins function as receptors for Neurexininduced postsynaptic differentiation. Additionally, this adhesion complex is cur- 
rently the only documented system to function in both excitatory and inhibitory synaptic differentiation (Graf et al., 2004).

The Neuroligin gene family is composed of four members in rodents encoding paralogous gene products of type-I transmembrane proteins NL1 (Ichtchenko et al., 1995), NL2, NL3 (Ichtchenko et al., 1996) and NL4 (Jamain et al., 2008). The paralogues share a conserved splice site-containing extracellular domain that mediates Neurexin binding and is homologous to acetycholinesterase (AChE) (Schema 4), making Neuroligins part of the $\alpha / \beta$-hydrolase fold superfamily (Hoffman et al., 2004). Neuroligin cytoplasmic domains are more divergent, though all share a C-terminal PDZ-binding motif with which they associate to MAGuKs, MAGIs and related proteins (Irie et al., 1997; Meyer et al., 2004). It is through their PDZ binding properties that Neuroligins are thought to mediate excitatory postsynaptic differentiation, with PDS-95 (Irie et al., 1997) and S-SCAM (lida et al., 2004) having both been proposed as immediate effectors.

Despite the extensive structural similarities shared by Neuroligin paralogues, their localization and function appear to be synaptically specialized. NL1 and NL2 segregate to excitatory and inhibitory synapses, respectively (Song et al., 1999; Varoqueaux et al., 2004), while NL3 appears to localize to subsets of both (Budreck et al., 2006). The synapse-specific localization of Neuroligins is mirrored by their functions in vivo since NL1 deletion results in aberrant glutamamtergic transmission (Chubykin et al., 2007) while NL2 deletion disrupts GABA- and glycinemediated transmission (Aramuni et al., submitted for publication). Complementing these data, in vitro experiments have indicated that Neuroligins along with excitatory and inhibitory scaffolding proteins can regulate the balance of excitation and inhibition (reviewed by Cline, 2005; Levinson \& El-Husseini, 2005).

The excitatory synaptic localization of NL1 does not appear to depend on its interaction with PDZ domain scaffolds (Dresbach et al., 2004), a notion reinforced by the fact that even inhibitory synapse-localized Neuroligins can associate with PDZ scaffolds (Meyer et al., 2004). Rather, the current evidence indicates that Neuroligin localization is determined by the presynaptic component. Splice site B in the NL1 extracellular domain contains a N-linked glycosylation site which determines specificity for presynaptic Neurexins. B site-containing NL1 interacts spe- 


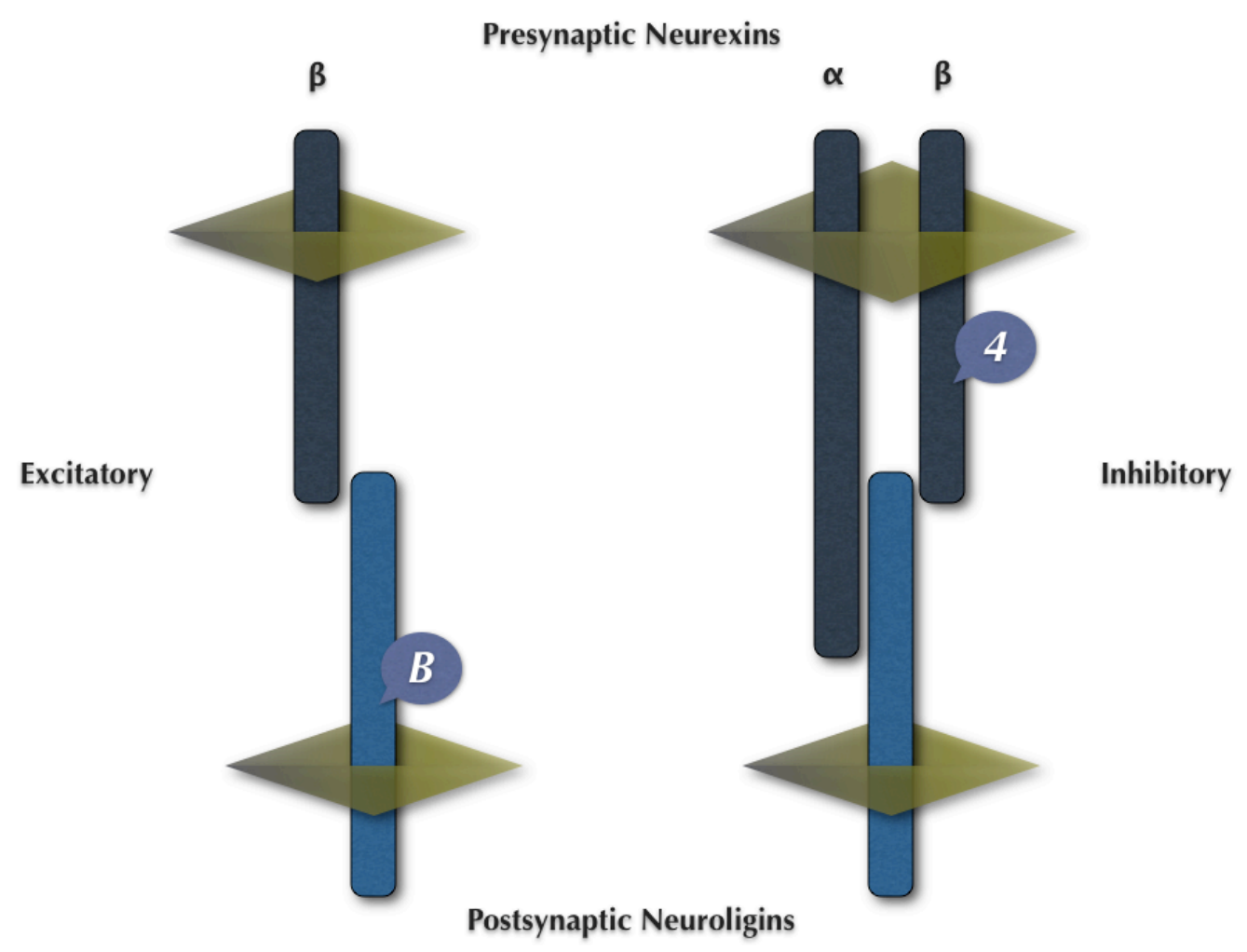

Schema 3: Splice-site determination of excitatory and inhibitory Neuroligin-Neurexin complexes. Neuroligins localized to excitatory synapses contain splice-site insert $B$ and bind to $\beta$ Neurexins lacking splice-site insert 4 . Neuroligins localized to inhibitory synapses lack splice-site $B$ and bind $\beta$-Neurexins containing splice-site insert 4 and $\alpha$-Neurexins. (Boucard et a. 2005; Graf et al. 2006; Chih et al. 2006).

cifically with $\beta$-Neurexins lacking splice site insert 4 . NL2, along with a rare variant of NL1 lacking splice insert B,preferentially associate with a-Neurexins and variants of $\beta$-Neurexins containing splice insert 4 (Schema 3; Boucard et al., 2005; Chih et al., 2006; Graf et al., 2006; Kang et al., 2007). However, this model has recently been contested (Chubykin et al., 2007).

Excitatory versus inhibitory selectivity being determined by transsynaptic interactions can be well-accommodated for by the notion of Neuroligins mediating specific synaptic differentiation, as transmitter compatibility is a major prerequisite for models of synaptic differentiation. However, the mechanisms involved in the transmission of specificity outside of the synaptic cleft are unclear. Association of Neuroligins with specific components of the inhibitory postsynapse has not been demonstrated, though NL2 has been shown to be able to recruit some of them (Graf et al., 2004). This, together with the promiscuous pairing of Neuroligins with excitatory scaffolds, confounds the proposal of a coherent model of synaptic dif- 
ferentiation that transcends the excitatory synapse and explains the function of Neuroligins in determining the balance of excitation versus inhibition.

\subsection{Aim of the study}

There is ample evidence that NL2 can induce inhibitory postsynaptic differentiation and is critical for inhibitory transmission in vivo. A minor role for other Neuroligins has also been documented. However, there is no evidence of a structural link of Neuroligins with components of the inhibitory postsynapse and, consequently, no mechanistic model of Neuroligin function thereat. The present study aims to identify the effectors of Neuroligin-mediated postsynaptic differentiation at inhibitory synapses. 


\section{Materials \& Methods}

\subsection{DNA constructs}

\subsubsection{Yeast-two-hybrid plasmids}

Yeast-two-hybrid bait constructs of rat full-length Gephyrin, NL1, NL2, NL3 and mouse NL4 cytoplasmic domains subcloned into the yeast expression vector pLexN were offered by Guido Meyer (Göttingen, Germany). The rat Dystroglycan cytosolic sequence was cloned from the commercial rat brain "QUICK-Clone" cDNA library (Clontech) from 8-12 week old prague-Dawley rats using flanking sequence primer sets in a polymerase chain reaction (PCR) with high fidelity pfu DNA polymerase (Stratagene) and a thermocycler protocol with the following key parameters:

\begin{tabular}{|l|l|}
\hline Annealing $\mathbf{T}\left({ }^{\circ} \mathrm{C}\right)$ & 66 \\
\hline Elongation $\mathbf{t}(\mathrm{sec})$ & 80 \\
\hline Iterations & 30 \\
\hline
\end{tabular}

Yeast-two-hybrid prey constructs (Table 2) were derived from screening an embryonic day 18 rat brain cDNA library in yeast expression vector pVP16-3 created by Masaya Okamoto (Dallas, Tx, USA) (Betz et al., 1997).

Mutant variants of the above constructs were produced employing full plasmid PCR amplification with 25-35 bp fully overlapping primers encompassing the site of mutagenesis and encoding the desired mutation(s). PCRs were performed with high fidelity pfu DNA polymerase (Stratagene) and thermocycler protocols with the following key parameters:

\begin{tabular}{|l|c|}
\hline Annealing $\mathrm{T}\left({ }^{\circ} \mathrm{C}\right)$ & 68 \\
\hline Elongation $\mathrm{t}(\mathrm{sec})$ & 120 per kb of plasmid \\
\hline Iterations & $12-15$ \\
\hline
\end{tabular}

Sequences of the complete open reading frames (ORFs) of the products were verified by DNA sequencing. 


\subsubsection{Expression plasmids}

Mammalian expression constructs of rat myc-NL1 and HA-NL2 in vector pcDNA3 (Invitrogen), and HA-NL3 in vector pCMV (Clontech Laboratories Inc.) were offered by Stéphane Jamain (Göttingen, Germany). Expression construct for human HA-CD8a in vector pNice was provided by Ann Marie Craig (Vancouver, BC, Canada). GFP-Gephyrin, CB2sH3-GFP, HA-CB2sH3- and HA-CB1 SH3+ $_{3}$ expression constructs were provided by Theofilos Papadopoulos and Heinrich Betz (Frankfurt, Germany). The Myc-CB2sH3+ expression construct was provided by Robert Harvey (London, UK). GABA $A$ receptor $\alpha 2, \beta 3$ and myc-tagged $\gamma 2$ subunit expression constructs were provided by Bernhard Lüscher (University Park, PA, USA). HACdc42/T17N and HA-Cdc42/Q61L mammalian expression constructs were provided by Heike Wegmeyer (Göttingen, Germany). Myc-S-SCAM expression construct was provided by Aleksandra Ivanovic (Göttingen, Germany). FLAG-NX1a expression construct was provided by Markus Missler (Göttingen, Germany).The membrane protein constructs above are tagged just downstream from the signal peptide sequence, yielding $\mathrm{N}$-terminal epitope tags that can be presented on the cell surface.

The CB2sH3+-GFP expression construct was engineered by restriction fragment swapping of a sequence in the Collybistin ORF encompassing the $\mathrm{SH} 3$ domain of $\mathrm{HA}-\mathrm{CB} 1_{\mathrm{SH}_{3}+}$ to the homologous Collybistin sequence flanking the splice site which omits the SH3 domain in the sequence of the $\mathrm{CB}_{\mathrm{SH} 3}-\mathrm{GFP}$ plasmid. The mycGephyrin expression construct was produced using the full-length prey clone of Gephyrin, corresponding to splice variant P1 (or Swiss-Prot isoform 5, containing splice cassettes $\mathrm{C} 2$ and $\mathrm{C} 6$ ), isolated from the yeast-two-hybrid screen by subcloning into pCMV-Myc vector. Fusion constructs of N-terminal enhanced Green Fluorescent Protein (GFP) and C-terminal yeast-two-hybrid prey fragments were engineered by standard subcloning of pVP16-3 inserts to the Sal I restriction site of the mammalian expression vector pEGFP-C1 (Clontech Laboratories Inc.).

$\mathrm{N}$-terminal Glutathione S-transferase (GST) fusion-protein expression constructs of the NL2 and Dystroglycan cytoplasmic domains were created by standard PCR sequence fragment amplification and subcloning into pGEX-4T1 bacterial expression vector giving rise to GST-NL2 $C D$ and GST-Dg $g_{C D}$ constructs. C-terminal human 
immunoglobulin1 heavy chain (Fc) fusion-protein constructs of the NL2 cytoplasmic domain were similarly created by subcloning into the mammalian expression vector pCMVIG9 (Ichtchenko et al., 1995). The product NL2cD-Fc expresses a cytoplasmic protein in mammalian cells.

Site-directed mutagenesis on several of the above constructs was performed as described in $\S 2.1 .1$.

\subsubsection{Cloning of full-length DRP2}

The full-length sequence of rat DRP2 was cloned in three sequence segments flanked by unique restriction sites. As DRP2 appears to have alternative start codons many of which appear to have potential Kozak consensus sequences, the 5' segment begins with AGAGACAGCC... 131 bp upstream of the first in-frame ATG initiating the largest ORF. That region is homologous to both DRP2 5' untranslated region (5'UTR) splice variants A and B (Roberts \& Sheng, 2000; GenBank Accession \#s AF195787 and AF195788), making it possible to prime either transcript. This segment is flanked by an engineered 5' Sal I site and a 3' natural codingsequence Cla I site. The central segment is flanked by natural coding-sequence Cla I and Xho I sites (positions 794 and 2353 of the largest ORF). The 3' segment begins at the Xho I site and and ends at an engineered Xba I site imediately downstream of the stop codon.

The 5' and the 3' segments were cloned using flanking sequence primer sets from the rat brain cDNA library (see $\S 2.1 .1$ ) using high fidelity pfu DNA polymerase (Stratagene) and thermocycler protocols with the following key parameters:

\begin{tabular}{|l|c|}
\hline Annealing $\mathbf{T}\left({ }^{\circ} \mathrm{C}\right)$ & 51 \\
\hline Elongation $\mathbf{t}(\mathrm{sec})$ & 135 \\
\hline Iterations & 30 \\
\hline
\end{tabular}

PCR products were subcloned into cloning vector pcR2.1-TOPO (Invitrogen) using the topoisomerase reaction after incubation with taq DNA polymerase (Sigma). The central segment was subcloned by restriction enzyme digest from the long prey fragment of DRP2 (89-828) in pEGFP-C1 and inserted into the cloning plasmid just downstream of the 5' segment. The 3' segment was subsequently sub- 
cloned downstream of the central segment in the cloning vector yielding a DRP2 sequence with a maximal ORF encoding a 957-residue polypeptide. Full-length DRP2 was subsequently subcloned into pcDNA3 (Invitrogen) to create a DRP2 mammalian expression construct.

\subsection{Antibodies \& labeling reagents}

\subsubsection{List of primary antibodies and working dilutions}

The following primary antibodies were employed throughout the study. In the following table Antigen refers to the protein or peptide epitope that was used to immunize hosts. Serum/clone refers to the serum designation number of polyclonal antibodies or clone designation number of monoclonal antibodies. Source refers to the commercial provider or creator of the antibody. Host refers to the species of animal an antiserum came from; monoclonal antibodies are designated as such and are derived from mouse. The last three columns of the table refer to the working dilutions employed in immunoblotting with enhanced chemiluminescence readout ( $E C L$ blot), in immunoblotting with infrared fluorescent readout ( $I R$ blot) or in immunocytochemistry labeling of cultured cells (ICC).

\begin{tabular}{|c|c|c|c|c|c|c|}
\hline Antigen & Serum / clone & Source & Host & ECL blot & IR blot & ICC \\
\hline$\beta$-Dystroglycan & 43DAG1/8D5 & Novocastra & monoclonal & $1: 100$ & & \\
\hline$\beta$-tubulin & & Sigma & monoclonal & & $1: 20000$ & \\
\hline c-myc & $9 \mathrm{E} 10$ & Sigma & monoclonal & $1: 500$ & & $1: 1000$ \\
\hline DRP2 & SA4625 & A. Poulopoulos & Rabbit & $1: 200$ & & \\
\hline DRP2 & SA4628 & A. Poulopoulos & Rabbit & $1: 200$ & & \\
\hline DRP2 & SA4629 & A. Poulopoulos & Rabbit & $1: 200$ & & \\
\hline FLAG & & Sigma & monoclonal & & & $1: 1000$ \\
\hline $\mathrm{GABA}_{\mathrm{A}} \mathrm{R}-\mathrm{a} 1$ & & J.M. Fritschy & Guinea Pig & $1: 10000$ & $1: 10000$ & \\
\hline $\mathrm{GABA}_{A} \mathrm{R}-\alpha 2$ & & J.M. Fritschy & Guinea Pig & $1: 1000-5000$ & & $1: 5000$ \\
\hline $\mathrm{GABA}_{A} \mathrm{R}-\beta 2 / 3$ & $\mathrm{BD} 17$ & Chemicon & monoclonal & $1: 100-250$ & & $1: 500$ \\
\hline $\mathrm{GABA}_{A} \mathrm{R}-\gamma 2$ & & Abcam & Rabbit & $1: 1000$ & & \\
\hline $\mathrm{GABA}_{A} \mathrm{R}-\gamma 2$ & & J.M. Fritschy & Guinea Pig & $1: 2000$ & $1: 500-1000$ & $1: 2500$ \\
\hline Gephyrin & 3B11 & Synaptic Systems & monoclonal & $1: 3500$ & $1: 2000$ & $1: 4000$ \\
\hline GFP & $7.1 \& 13.1$ & Roche & monoclonal & $1: 2000$ & & \\
\hline GFP & & Abcam & Rabbit & $1: 3500$ & & \\
\hline GFP & & Synaptic Systems & Rabbit & $1: 7000$ & & \\
\hline
\end{tabular}




\begin{tabular}{|c|c|c|c|c|c|c|}
\hline Antigen & Serum / clone & Source & Host & ECL blot & IR blot & ICC \\
\hline GlyR-alpha & $4 a$ & Synaptic Systems & monoclonal & $1: 1000$ & & \\
\hline $\mathrm{HA}$ & SG77 & Zymed & Rabbit & $1: 2000$ & & $1: 2000$ \\
\hline $\mathrm{HA}$ & 12CA5 & Roche & monoclonal & $1: 7000$ & & $1: 2000$ \\
\hline HA.11 & 16B12 & Covance & monoclonal & $1: 3500$ & & $1: 2000$ \\
\hline Neuroligin 1 & $4 \mathrm{C} 12$ & N. Brose & monoclonal & $1: 10000$ & $1: 5000$ & $1: 2000$ \\
\hline Neuroligin 2 & 799 & F. Varoqueaux & Rabbit & $1: 2000-7000$ & & $1: 2000$ \\
\hline Neuroligin 3 & 804 & F. Varoqueaux & Rabbit & $1: 1000-7000$ & $1: 500$ & $1: 500$ \\
\hline Neuroligin 4 & 75 & S. Jamain & Rabbit & $1: 1000$ & $1: 1000$ & \\
\hline NMDA R1 & M68 & Synaptic Systems & monoclonal & $1: 7000$ & & \\
\hline Synapsin $1 / 2$ & & Synaptic Systems & Rabbit & & & $1: 2000$ \\
\hline Synaptophysin & 7.2 & Synaptic Systems & monoclonal & & $1: 10000$ & \\
\hline vGlut1 & & Synaptic Systems & Rabbit & & $1: 2000$ & $1: 1000$ \\
\hline vGlut2 & & Chemicon & GuineaPig & & $1: 2000$ & $1: 2500$ \\
\hline vIAAT & & Chemicon & Rabbit & $1: 500$ & & $1: 2000$ \\
\hline
\end{tabular}

\subsubsection{Conjugated antibodies and reagents}

Goat-derived antibodies with species-specific affinities for immunoglobulins conjugated to horseradish peroxidase (Bio-Rad and Jackson Biochemicals) were used for ECL immunoblotting at a dilution of 1:10000. IRDye 700 and 800 fluorophores (Rockland Immunochemicals) were used for IR immunoblotting at a dilution of 1:5000 or Cy5, Alexa Fluor 488 and 555 fluorophores were used for immunocytochemistry at a dilution of 1:1000. Alexa Fluor 350- or Oregon Green-conjugated phalloidin was used to label Actin in fixed cells at a dilution of 1:50.

\subsubsection{Production of anti-DRP2 antisera}

Two peptide epitopes from the rat DRP2 sequence were selected based on their predicted antigenicities, surface exposure and lack of similar sequences in other proteins encoded in the rodent genomes. Animal care, peptide production, host immunization and bleeding were performed by Eurogentec. Epitope TPDTEVADDVGSKSQ (corresponding to DRP2 amino acid sequence 910-924) with an additional C-terminal cystein residue for conjugation gave rise to rabbit antisera SA4628 and SA4629. Epitope SAEATPDHRNE (corresponding to DRP2 amino acid sequence 823-833) with an additional C-terminal cystein residue for conjuga- 
tion gave rise to rabbit antiserum SA4624. The antisera employed herein were derived from the final bleeding of the animals.

\subsection{Yeast-two-hybrid assays}

The LexA yeast-two-hybrid system was used for small scale assays with a selection of bait and prey constructs. Bait constructs are in yeast expression vector pLexN, fusing the DNA-binding region of LexA and a SV40 large T-antigen nuclear localization signal to the inserted sequence. Prey constructs are in yeast expression vector pVP16-3, fusing the VP16 acidic activation domain to the inserted sequence. Interaction of prey and bait fusion constructs will lead to a functional transcription activation complex and to reporter gene expression under the control of the LexA promoter.

Assays were performed using small-scale co-transformation with the indicated bait and prey constructs along with Salmon sperm carrier DNA by a lithium acetate method, as described in Betz et al. 1997, into auxotrophic Saccharomyces cerevisiae strain L40, lacking metabolic genes for tryptophane, leucine and histidine production. Transformants were plated on media lacking tryptophane and leucine and incubated at $30^{\circ} \mathrm{C}$ for 3 days. Prototropic colonies that emerged represented clones transformed for both bait and prey constructs.

Prey-bait interaction leads to the expression of reporter genes under the control of LexA. Interaction was assessed in co-transfected clones examining for $\beta$ galactosidase activity of LacZ reporter gene expression (Vojtek et al., 1993). The readout is based on the catabolism of the chromogenic compound X-gal making $\beta$ galactosidase activity visible. 


\subsection{Cell culture and transfection}

\subsubsection{Cell-Line culture and transfection}

The COS7 cell-line, a fibroblast line derived from African green monkey kidney cells, and the HEK 293FT cell-line, an epithelial line derived from human embryonic kidney cells transformed with the large T-antigen, were used in this study. Both cell-lines were maintained plastic tissue culture dishes with high-glucose Dulbecco's Modified Eagle's Medium (DMEM) (Gibco) supplemented with 10\% fetal calf serum (Gibco) in $37^{\circ} \mathrm{C}$ humid incubator with $5 \%$ ambient $\mathrm{CO}_{2}$. Selection agent $\mathrm{G} 418$ at a concentration of $500 \mu \mathrm{g} / \mathrm{ml}$ was added to the culture medium of HEK 293FT cells. Passaging was performed using standard procedures of trypsinmediated dislodgment of confluent cultures. Transfections were performed using the FUGENE6 (Roche) reagent by standard lipofection procedures.

\subsubsection{Primary neuron culture}

Primary neuron cultures were prepared from hippocampi of embryonic day 18 rats or neonatal mice. Rat hippocampi were treated with trypsin (Gibco) in HBSS (Gibco) for $30 \mathrm{~min}$ and mechanically triturated. Cells were plated on plastic tissueculture dishes or poly-D-lysin-coated glass coverslips at a density of 31600 cells/ $\mathrm{cm}^{2}$. Mouse hippocampi were treated with papain (Worthington) in HBSS for 30 min and mechanically triturated. Cells were plated on a continental astrocyte layer on poly-D-lysin-coated glass coverslips at a density of $31600 \mathrm{cells} / \mathrm{cm}^{2}$ for transfection and at 15800 cells $/ \mathrm{cm}^{2}$ otherwise. Neurons were cultured in Neurobasal medium (Gibco) supplemented with B27 (Gibco), glutamax (Gibco), and Penicillin/ Streptomycin (Roche). 


\subsection{Recombinant protein binding assays}

\subsubsection{GST-fusion proteins}

To express NL2 and Dystroglycan cytoplasmic domain fusion proteins with Nterminal GST, BL21DE3 strain E. coli were subjected to electroporation-mediated plasmid transformation with the respective constructs in pGEX-4T1. Luria broth cultures of transformants in the logarithmic growth phase were induced for recombinant protein expression with $0,5 \mathrm{mM}$ IPTG for $4 \mathrm{~h}$ at room temperature. The culture was subsequently harvested and cells lysates were prepared by a combination of catabolic enzyme treatment with Lysozyme and DNase, and sonication with a Labsonic U Sonifier (Braun). A standard mix of Leupeptin $1 \mu \mathrm{M}$, Aprotinin $1 \mu \mathrm{g} /$ $\mathrm{ml}$ and PMSF $100 \mu \mathrm{M}$ protease inhibitors was omnipresent in these and all subsequent cell homogenization or lysis procedures.

Lysate supernatants were used as input for standard glutathione-affinity purification. Glutathione coupled to Sepharose beads (Amersham) was incubated with the input samples according to standard batch method purification protocols. To determine protein expression and purity, beads were eluted with glutathione and samples were subjected to standard SDS Polyacrylamide Gel Electrophoresis (SDS-PAGE) in tris-glycine gels and in-gel protein staining with Coomassie blue stain.

Beads loaded with purified protein were used directly for GST-fusion protein binding assays. As input for these assays, transfected HEK 293FT cells expressing cytoplasmic GFP-fusion proteins were homogenized using rotating teflon potters or a cell cracker (EMBL), with 5-10 $\mu \mathrm{M}$ clearance, in standard Tris/HCl-based buffers without the use of detergents. Beads were incubated at varying conditions with high-speed supernatants of the above homogenates and reclaimed using batch methods. Elution of bound proteins was performed directly in Laemmli sample buffer (LSB). Samples were prepared on blots by standard SDS-PAGE in trisglycine gels and nitrocellulose electroblot methodologies. Blots were examined for the presence of GFP-fusion proteins by anti-GFP immunoblotting. 


\subsubsection{Fc-fusion proteins}

Transfected HEK 293FT cells expressing NL2 cytoplasmic domain fused Cterminally to Fc were homogenized as described in $\S 2$ 2.5.1. FC-fusion proteins were purified using Protein-A coupled to Sepharose beads (Amersham) following standard batch method. Protein expression and purity were determined after elution of proteins bound to beads directly in LSB and subsequent standard SDSPAGE in tris-glycine gels and in-gel protein staining with Coomassie blue stain.

Fc-fusion protein binding assays were performed with GFP-fusion proteins equivalently to the procedures described in $\S 2.5 .1$ using purified Fc-fusion proteins directly on the Protein A coupled Sepharose beads. Alternatively, Fc- and GFPfusion proteins were co-expressed in the same culture. The purification procedure described above was used as a co-precipitation assay by examining the purified material for GFP-fusion proteins by immunoblotting. Detection of immunoreactive bands was performed by ECL.

\subsubsection{Co-immunoprecipitation in cell-lines}

HEK 293FT cells plated on $10 \mathrm{~cm}$ dishes and expressing HA-tagged constructs together with GFP-fusion proteins were lysed $24 \mathrm{~h}$ post transfection in TNE buffer with $1 \%$ non-denaturing detergent (TritonX-100, Nonidet P-40 n-Ocly- $\beta D$ glucoside or Cholate). Lysates were centrifuged to obtain clear extracts, which, in turn, were incubated with antibodies for 4 to $14 \mathrm{~h}$ at $4^{\circ} \mathrm{C}$. Immunoreactive complexes were retrieved using Protein A for polyclonal antibodies, or protein $G$ for monoclonal antibodies, coupled to Sepharose beads (Amersham). Beads were washed three times with buffer containing $0,5 \%$ to $1 \%$ detergent and once with buffer alone. Beads were eluted in LSB providing samples for SDS-PAGE in tris glycine gels and standard immunoblotting with rabbit-anti-GFP (Abcam) or monoclonal anti-myc 9E10 (Sigma) antibodies. Detection of immunoreactive bands was performed by ECL. 


\subsection{Crosslinking and identification of protein com- plexes}

\subsubsection{Preparation of crosslinked brain material}

Crosslinking was performed on post-nuclear homogenates from a single adult mouse brain in phosphate buffer saline (PBS). Homogenates were incubated on ice for 20 min with $200 \mu \mathrm{M}$ of the cleavable, homobifunctional, membrane permeable, $12 \AA$ crosslinker Dithiobis[succinimidylpropionate] (DSP) (Pierce). Crosslinker was quenched in Tris buffer before the material was centrifuged at $21000 \mathrm{~g}$ for $15 \mathrm{~min}$. Proteins from pelleted membranes were extracted from pellets with 1\% SDS in TNE buffer (Tris $50 \mathrm{mM}, \mathrm{NaCl} 150 \mathrm{mM}$, EDTA $5 \mathrm{mM}$, Leupeptin 1 $\mu \mathrm{M}$, Aprotinin $1 \mu \mathrm{g} / \mathrm{ml}$, and PMSF $100 \mu \mathrm{M}$ ).

\subsubsection{Preparations with in situ crosslinking of cell-line cultures}

COS7 cells, plated on 10 or $6 \mathrm{~cm}$ diameter tissue culture dishes were treated in situ with crosslinker $16 \mathrm{~h}$ post-transfection. For stoichiometric surface crosslinking, cells were treated with $100 \mu \mathrm{M}$ of the cleavable, homobifunctional, membrane impermeable, $12 \AA$ crosslinker 3,3' Dithiobis[sulfosuccinimidylpropionate] (DTSSP) (Pierce) for 10 min on ice in PBS supplemented with $5 \mathrm{mM}$ EDTA. For cytoplasmic crosslinking, cells were treated for 20 min on ice with $1 \mathrm{mM}$ DSP in PBS. The crosslinker was subsequently quenched in TNE and cells were directly lysed with TNE containing $1 \%$ SDS. Lysates were passaged four times through a needle and syringe to shear DNA and centrifuged to obtain SDS extracts.

\subsubsection{Preparations with in situ crosslinking of neuron cultures}

Day in vitro (DIV) 14 hippocampal rat neurons cultured on poly-D-lysine-coated plastic culture dishes at a density of 31600 cells per $\mathrm{cm}^{2}$ for a total 890000 cells per sample were treated in situ with $100 \mu \mathrm{M}$ of the non-cleavable, homobifunctional, membrane impermeable, 11,4 $\AA$ crosslinker Bis(sulfosuccinimidyl)suberate (BS) (Pierce) in PBS supplemented with $5 \mathrm{mM}$ EDTA for $20 \mathrm{~min}$ on ice. 
Crosslinker was quenched in TNE buffer and cells were lysed in TNE with 1\% SDS. Lysates were passaged four times through a needle and syringe to shear DNA and centrifuged to obtain SDS extracts.

\subsubsection{Immunoprecipitation of crosslinked complexes}

SDS extracts of crosslinked proteins were diluted with 7 volumes of TNE with $1 \%$ TritonX-100, producing a mixed micelle system of $0,875 \%$ TritonX-100 and $0,125 \%$ SDS that does not denature antibodies. Neuroligin-containing crosslinked adducts were immunoprecipitated from neuron or brain material with paraloguespecific antibodies listed in $\S$ 2.2.1. HA-tagged proteins were immunoprecipitated with the antibody $16 \mathrm{~B} 12$. After overnight incubations at $4^{\circ} \mathrm{C}$ of the extracts with antibodies, IgG containing complexes were recovered by a $5 \mathrm{~h}$ incubation at $4^{\circ} \mathrm{C}$ with Protein A for polyclonal antibodies, or Protein G for monoclonal antibodies, coupled to Sepharose beads. Beads were washed six times in 0,875\% TritonX-100 / 0,125\% SDS TNE buffer and proteins were eluted in LSB with 7,5\% $\beta$ mercaptoethanol to reduce protein disulfide bonds and cleave the crosslinker when DSP was used. Samples were subjected to standard SDS-PAGE on trisglycine gels and immunoblotted on nitrocellulose membranes using antibodies from $\S 2.2 .1$ to detect crosslinked complex components. For stoichiometric crosslinked adduct analysis, BS $^{3}$ non-cleavable linker samples were reduced with $7,5 \% \beta$-mercaptoethanol, while DTSSP cleavable linker samples were left unreduced. Samples were analysed by SDS-PAGE on Tris-Acetate precast $3 \%$ to $8 \%$ gradient gels (Invitrogen) along with high molecular weight protein standards (Invitrogen).

\subsection{Synaptic plasma membrane preparation}

Forebrain and cerebellum from adult NL2-deficient and wild-type mice were collected. Each sample represented pooled material from three mice, while three samples were prepared from wild-type mice and two from NL2-deficient mice. Genotyping of the mice was performed by PCR with wild-type and mutant specific primers for the Nign2 gene on DNA prepared from mouse tail samples using tail- 
prep DNA kit (Nexttec). Homogenates and synaptic plasma membranes (SPM) were prepared based on previously described protocols (Jones \& Matus, 1974). Material was homogenized in $320 \mathrm{mM}$ sucrose at $4^{\circ} \mathrm{C}$ with 13 strokes in a glass and teflon potter rotating at $900 \mathrm{rpm}$. Post-nuclear homogenates, corresponding to the supernatants of an $800 \mathrm{~g}$ centrifugation for $10 \mathrm{~min}$, were centrifuged at $9200 \mathrm{~g}$ for $15 \mathrm{~min}$. The resulting pellet, corresponding to the crude synaptosomal fraction ,was resuspended in water for osmotic lysis, washed, and centrifuged at $25000 \mathrm{~g}$ for $20 \mathrm{~min}$. Synaptic plasma membranes (SPMs) were enriched on a 0,32-1,2 M sucrose gradient by centrifugation at $19000 \mathrm{~g}$ for $180 \mathrm{~min}$. The SPM fraction, corresponding to the opaque material at the 0,8-1,2 $\mathrm{M}$ sucrose interphase, was collected as a pellet after washing with buffer and centrifugation at $37,000 \mathrm{~g}$ for 20 $\min$.

Protein levels were determined in homogenate and SPM samples using the BCA method (Pierce) and samples were adjusted to $2 \mathrm{mg} / \mathrm{ml}$ protein. Samples were analyzed by SDS-PAGE in tris-glycine gels and immunoblotted with antibodies diluted as noted in $\S 2.2 .1$. Protein levels in the samples were assessed by fluorophore-coupled secondary antibodies and infrared band intensity quantification on an Odyssey fluorescence reader (Li-Cor). Each lane was labeled in parallel for $\beta$-Tubulin as a reference protein, and homogenate levels were normalized to the average of the reference protein in all homogenate samples. Enrichment of proteins in the SPM fraction was expressed as a ratio of SPM to normalized homogenate levels.

\subsection{Immunolabeling and surface clustering assays}

\subsubsection{Immunolabeling of neurons and transfected cell-lines}

Transiently transfected COS7 cells were plated directly onto glass coverslips while HEK-296 FT cells were plated onto poly-D-lysine coated glass coverslips. Hippocampal neurons from mice genotyped for Nlgn2 as noted in $\S 2.7$ were plated on continental astrocyte layers on poly-D-lysin-coated glass coverslips at a density of 15.800 cells $/ \mathrm{cm}^{2}$. Cells were fixed 12 to 24 hours post transfection in 4\% PFA, 
5,5\% sucrose in $100 \mathrm{mM}$ phosphate buffer $\mathrm{pH} \mathrm{7,4}$ (PB) for 10 minutes at room temperature. Samples were permeabilized and blocked with 0,1\% TritonX-100, 5\% normal goat serum and $0,1 \%$ gelatin in PB. Cells were stained with primary antibodies in $5 \%$ normal goat serum, gelatin $0,1 \%$ in PB overnight at $4^{\circ} \mathrm{C}$. Secondary antibody staining was similarly performed for 1 hour at room temperature using anti-isotypic fluorophore-conjugated antibodies.

Cell-line samples were imaged using an inverse Leica DMIRE2 microscope equipped with an HCX PL APO 63x oil-immersion objective lens and connected to a Leica TCS SP2 AOBS confocal laser scanning setup. Intensity correlation analysis was performed on the images using the ImageJ software $(\mathrm{NIH})$. Briefly, a Gaussian blur was applied to the images using a kernel size of 1 pixel. The HA channel was thresholded to omit pixels coming from the extracellular field. Standard Pearson's correlation coefficient was evaluated in the thresholded fields using the Intensity Correlation Analysis plugin for ImageJ from Tony Collins (Wright Cell Imaging Facility, Toronto, Canada) and Elise Stanley (C\&MB, TWRI, Toronto, Canada).

Analysis of Gephyrin distribution in immunolabeled mouse hippocampal cultures from NL2-deficient and wild-type animals was carried out on an Olympus BX-61 upright epifluorescent microscope equipped with a $63 \mathrm{X}$ oil-immersion objective and an Esivision CCD camera coupled to the AnalySIS image acquisition software (Olympus). Synaptic Gephyrin clusters on the somatic plasma membrane were designated by thresholding of Gephyrin immunoreactivity and apposition to thresholded Synapsin immunoreactivity. Cytoplasmic Gephyrin aggregates in neurons were distinguished by their characteristic sphericity, large size, and saturation of fluorescence on acquired images. Quantification of somatic postsynaptic Gephyrin clusters and of the occurrence of cytoplasmic Gephyrin aggregates was performed manually on DIV16 hippocampal neuron cultures.

\subsubsection{Acute surface clustering of transfected neurons and cell-lines}

COS7 cells cultured on glass coverslips and transiently co-transfected with HAtagged membrane protein and GFP-fusion cytoplasmic constructs were subjected to HA surface clustering $24 \mathrm{~h}$ post transfection. Cells were treated at room tem- 
perature in PBS containing 10\% normal goat serum. Cells were first left for 30 min without additional treatment. Subsequently cells were treated with 1:100 diluted rabbit-anti-HA antibody (Zymed) for $1 \mathrm{~h}$, washed, treated with 1:266 diluted Alexa633 conjugated goat-anti-rabbit antibody, washed and fixed. HA clustered immunofluorescence and GFP fluorescence were imaged on an Olympus BX-61 upright epifluorescent microscope equipped with a $63 \mathrm{X}$ oil-immersion objective and an Esivision CCD camera coupled to the AnalySIS image acquisition software (Olympus).

Hippocampal neurons from NL2-deficient mice were transfected with HA-tagged membrane protein constructs at DIV7 using Lipofectamine2000 (Invitrogen) and $500 \mathrm{ng}$ plasmid DNA per $1,9 \mathrm{~cm}^{2}$ well for 4 hours before being transferred to their normal medium. Surface clustering of HA-tagged proteins was achieved after treatment of DIV8 transfected neurons with $5 \mathrm{ng} / \mu \mathrm{l}$ monoclonal $\lg _{2 b}$ anti-HA antibody $12 \mathrm{CA} 5$ for 25 minutes followed by treatment with $2,5 \mathrm{ng} / \mu$ l fluorophoreconjugated isotype-specific goat-anti-mouse $\lg _{2 b}$ antibody (Molecular Probes) in culture medium at room temperature prior to fixation. Samples were washed, fixed

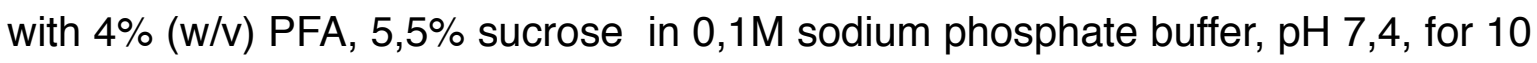
min, permeabilized for 5 min with $0,1 \%$ triton-X100 in PBS containing 5\% normal goat serum, and incubated 3 hours at room temperature with combinations of primary antibodies to monoclonal $\lg \mathrm{G}_{1}$ mouse-anti-gephyrin 3B11 and rabbit-antisynapsin 1/2. After further washes, the neurons were incubated with fluorophoreconjugated goat-anti-rabbit and isotype-specific goat-anti-mouse $\lg _{1}$ secondary antibodies (Molecular Probes).

Samples were imaged on an Olympus BX-61 upright epifluorescent microscope equipped with a $63 \mathrm{X}$ oil-immersion objective and an Esivision CCD camera coupled to the AnalySIS image acquisition software (Olympus). To quantify extrasynaptic HA-tagged protein - Gephyrin co-clustering, three-channel images were acquired using the same acquisition settings on all transfected neurons detected (for HA-NL2 $n=29$, HA-NL2. Y770A n=31, HA-CD8a $n=23$ and HA-CD8a $n=12$ ). Images were analyzed with the ImageJ software as follows: Synaptic particles were designated by application of a common intensity threshold on the Synapsin channel. Particles surpassing threshold were dilated to include the surrounding pixels. HA or Gephyrin immunoreactivity within these particles was excluded from 
further analysis as was considered to represent synapse-associated clusters. Acutely induced non-synaptic HA particles were designated by a similar thresholding process on the HA channel after subtraction of the synaptic regions and with additional criteria of size and circularity so as to exclude circumstantial large particles arising from out of focus HA immunoreactivity surpassing threshold. All HA particles detected (for HA-NL2 $n=4690$, HA-NL2.Y770A $n=5712$, HA-CD8a $n=4830$ and HA-CD8a $n=2224$ ) were considered in subsequent analysis to determine the intensity ratio of endogenous gephyrin versus HA cluster immunoreactivity.

\subsection{Statistical analysis}

Statistical analyses were performed using unpaired, two-tailed Student's T-test. Variance is expressed as standard error of the mean. 


\section{Results}

\subsection{Investigative analysis of novel cytosolic interaction partners of Neuroligins}

Neuroligins appear to have a role in the protein recruitment events during the formation of the postsynaptic specialization. In order to study how Neuroligins participate in these processes at inhibitory synapses, the Neuroligin 2 (NL2) protein was selected for study, as this Neuroligin family member has consistently and exclusively been observed at inhibitory postsynapses (Varoqueaux et al., 2004). The experimental goal of this study was to identify cytosolic proteins that are recruited to postsynaptic sites of inhibitory terminals by associating with NL2. A list of potential such proteins was compiled employing a yeast-two-hybrid screening methodology (Poulopoulos et al., submitted for publication).

Previous yeast-two-hybrid screens with the cytoplasmic domain (CD) if Neuroligins have been dominated by PDZ domain-mediated interactions (Neeb, 2003; Irie et al., 1997; Meyer et al., 2004), yet PDZ domain-containing proteins are not prominent components of inhibitory postsynapses. In order to enhance the probability of identifying proteins that are relevant to inhibitory synapses, the PDZ domain bias of the yeast-two-hybrid system was superseded. cDNA sequences of the NL2 CD were isolated from rat brain material and genetically engineered to lack the codons

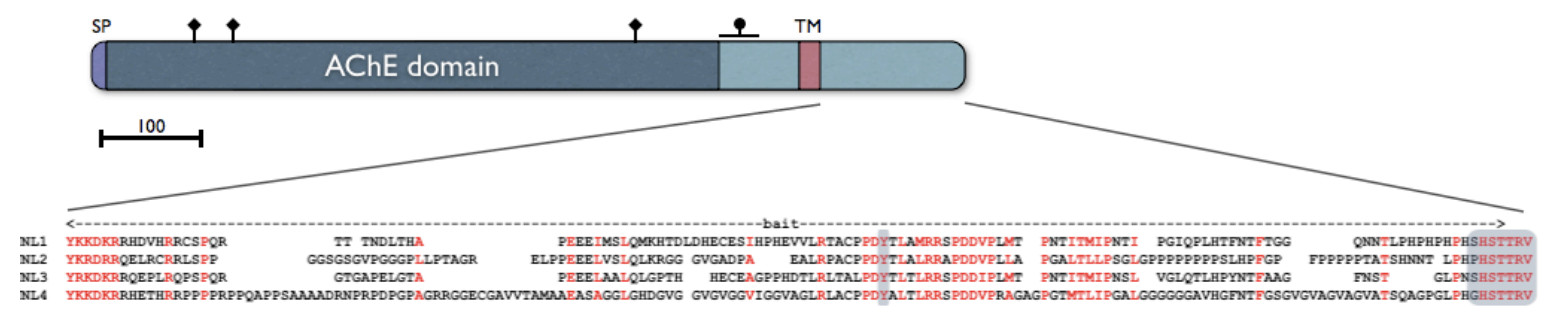

Schema 4: Neuroligin domain structure. Neuroligins are membrane proteins with a N-terminal signal peptide (SP) and one transmembrane domain (TM). Most of the extracellular domain is homologous to AChE. N-linked glycosylation sites are denoted with diamonds. The O-linked glycosylation region is denoted with a circle. The cytoplasmic domain is enlarged to show the primary sequences of the four rodent Neuroligin paralogues in alignment. The C-terminal PDZbinding motif and the tyrosine residue critical for binding Gephyrin are highlighted. The portion of the cytoplasmic domain of NL2 used for the yeast-two-hybrid screen is noted above. Scale bar represents amino acid residues. 
for the three extreme C-terminal amino acid residues (NL2 $C D-\triangle P D Z$, Schema 4). The resulting protein product is predicted to loose its affinity for binding PDZ domains (Meyer et al., 2004). DNA sequences were further engineered so that the resulting yeast protein would comprise a chimaera of the NL2CD- $\triangle \mathrm{PDZ}$ sequence and the DNA-binding domain of LexA including an additional element that conforms to the consensus sequence for nuclear localization signals.

This DNA construct was used to express a protein in yeast that would function as a yeast-two-hybrid bait when co-expressed with fusion proteins containing the transcription initiating activity of VP-16, functioning as the yeast-two-hybrid prey. For the purposes of the described screen, a library of neonatal rat brain cDNA fused to VP-16 activator (Betz et al., 1997) was screened in L40 strain reporter yeast containing the NL2CD- $\triangle \mathrm{PDZ}$ bait construct described.

The screen yielded a considerable number of yeast clones showing positive reporter gene activity. 505 of these were cloned and sequenced using TempliPhi sequencing (Reagin et al., 2003). From the prey clones identified, Table 2 presents a list of unique candidates that were considered plausible cytosolic interactors of NL2. Of these 30 proteins, 11 contain predicted proline-binding domains, namely WW and $\mathrm{SH} 3$ domains. This observation appears to reflect the proline-rich nature of the NL2 CD (Schema 4).

Table 2: List of unique non-PDZ NL2 cytoplasmic interaction partners from the yeast-two-hybrid screen.

\begin{tabular}{|ccc|}
\hline a-actinin & FBP11 & POSH \\
Abi-1 & Fe65 & POSH-like \\
Aida & Fe65-like & Profilin II \\
ArgBP2 & GAS7 & Rapostlin \\
B-IV Spectrin & Gephyrin & Sca I \\
B-Catenin & Groucho & Spinophilin \\
DAAM1 & Kalirin & S-SCAM \\
DRP2 & Magi-1 & SynGAP \\
EB1 & Magi-3 & TOCA-1 \\
Endophilin & Nedd4 & VASP \\
\hline
\end{tabular}


This list (Table 2) was the starting point of an analysis meant to identify those candidates which were most relevant in regard to the aims of the present study. Subsequent analysis was designed to select candidates that most likely represent proteins that fulfill the following criteria: a) proteins are actual binding partners of NL2 in vivo, b) the interaction takes place at the inhibitory postsynaptic membrane, and c) the interaction is involved in the deployment of the postsynaptic apparatus.

\subsubsection{Yeast-two-hybrid analysis of putative cytosolic interaction part- ners of Neuroligin 2}

To gain insight into the plausibility of any given putative interaction partner of NL2 to function at the inhibitory postsynapse, a series of cross-screens was designed using reporter gene activation as readout of the yeast-two-hybrid system. Collective information on possible additional interaction properties of the candidates was obtained by assaying the 30 prey constructs versus a selection of additional bait constructs.

\subsubsection{Cross-screen: components of the inhibitory postsynapse}

To examine potential association of the candidates with other inhibitory postsynaptic components, two proteins that are known to specifically associate with inhibitory postsynapses were selected to cross-screen. The CD sequence of Dystroglycan (DG), an adhesion protein that appears in a subset of mature inhibitory postsynapses (Lévi et al., 2004), and the full-length sequence of Gephyrin, the constitutive postsynaptic scaffolding molecule of inhibitory synapses, were used to design prey constructs following the general structure of the NL2 CD bait construct of the initial screen. Interaction of the prey fusion proteins with either of the bait fusion proteins was assayed for LacZ reporter gene activation (Table 2).

The DG CD interacted in yeast with a prey fusion protein of Dystrophin Related Protein 2 (DRP2), in accord with a previous yeast-two-hybrid report (Sherman et al., 2001). This interaction was subsequently confirmed using a GST pulldown ap- 


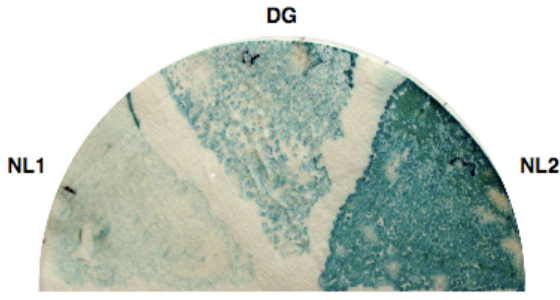

S-SCAM (WW)
Figure 1: Yeast-two-hybrid assay of S-SCAM (WW) against NL1, NL2 and DG. S-SCAM prey fragment encompassing the two WW domains interacts preferentially with $\mathrm{NL} 1<\mathrm{DG}<\mathrm{NL} 2$ cytoplasmic domains in yeast.

proach (data not shown). Apart from DRP2, a Synaptic Scaffolding Molecule (SSCAM) fragment encompassing the two WW domains was also observed to interact with the CD of DG. This came as a surprise since S-SCAM has been shown to interact with NL1 at excitatory synapses (Hirao et al., 1998; lida et al., 2004). The fact that S-SCAM is observed in yeast to interact with the inhibitory adhesion proteins NL2 and DG (Figure 1) in a PDZ domain-independent manner may imply a role for this scaffolding molecule at the inhibitory postsynapse. These initial observations have been subsequently confirmed with biochemical and cell biological data and it appears that S-SCAM does indeed localize to a subset of inhibitory synapses where it interacts with DG (Sumita et al., 2006).

The Gephyrin bait fusion protein interacted in yeast only with the prey fusion protein containing the Gephyrin sequence. This observation, consistent with previous reports of Gephyrin oligomerization (Sola et al., 2004), confirms that the Gephyrin bait clone expresses and is functional in yeast. Surprisingly, the other two NL2 interaction partner candidates that have previously been reported to interact with Gephyrin, namely Profilin II and VASP (Mammoto et al., 1998; Giesemann et al., 2003) did not exhibit LacZ activation when co-expressed with the Gephyrin bait fusion protein. As yeast-two-hybrid assays were not used in the previous studies, this discrepancy may arise from the difference in experimental systems employed.

\subsubsection{Cross-screen: Neuroligin paralogues}

Paralogous comparison of the four Neuroligin genes shows extensive homology with identity in the range of $50 \%$. Despite this homology, Neuroligin paralogues display distinct synapse specificity as they are observed to segregate between excitatory and inhibitory synapses (Song et al., 1999; Varoqueaux et al., 2004). With less than $30 \%$ identity, the CD of the proteins however is more divergent than the 


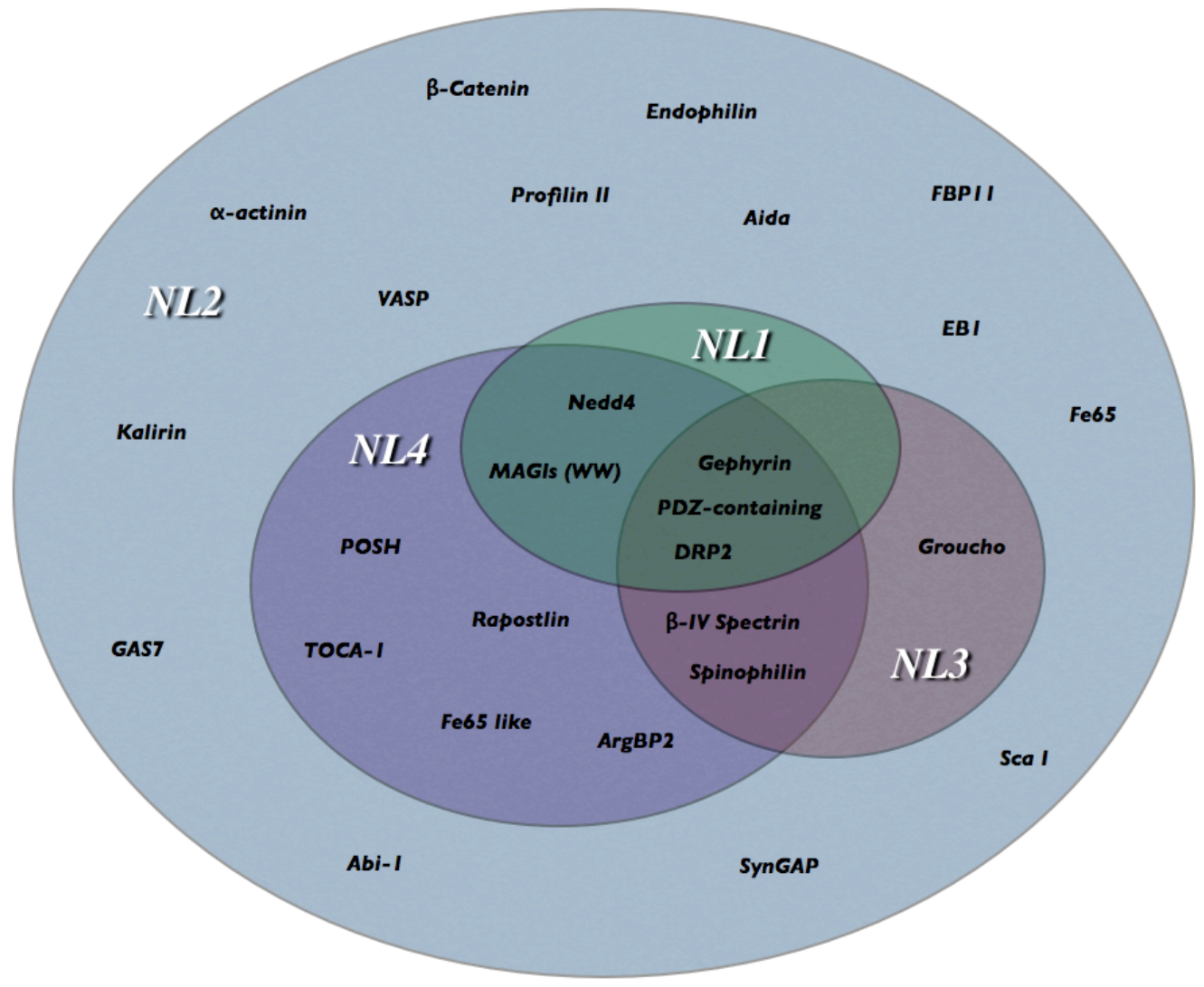

Schema 5: Putative cytoplasmic interaction partners of Neuroligins. Circle diagram showing the overlapping Neuroligin paralogue specificities of putative cytoplasmic interaction partners of NL2 as seen by LacZ reporter gene activation readouts in yeast-two-hybrid assays.

other regions. In the context of the divergent $C D$ sequences and distinct synaptic targeting, the question of how NL2 putative interaction partners distribute in regard to their ability to bind other Neuroligin paralogues was addressed.

The full CDs of rat NL1, 2 and 3 and mouse NL4 were cloned into bait constructs and coexpressed with the candidates from the screen. Schema 5 summarizes the results of LacZ reporter gene activation assays, and displays that putative NL2 cytosolic interaction partners can be divided into categories depending on their NLbinding properties. 14 of the candidate interactors showed exclusivity to NL2, while PDZ domain-containing proteins (Meyer et al., 2004), DRP2 and Gephyrin exhibited promiscuous binding to all Neuroligin paralogues. Interestingly, the remaining candidates show eclectic binding to specific Neuroligin paralogue combinations.

\subsubsection{Cross-screen: Neuroligin mutants}


For subsequent analyses of the putative interactions of the yeast-two-hybrid screen candidates with NL2, binding-deficient mutants would provide a valuable tool. Ensuring that such mutants behave as negative controls in the different assay systems would indicate that the readouts of these different assays can be considered as examining phenomenons that are mechanistically equivalent. Such mutants would also serve as loss-of-function variants of NL2. Finally, such mutations would point to critical residues potentially demarcating the interacting epitope and facilitating binding-site mapping of selected candidates.

To avoid independent binding-site mapping for each of the 30 candidates prior to evaluation of which ones present interest for the formation and function of the inhibitory postsynapse, residues or regions of the NL2 CD that appeared to potentially be part of stereotypized binding motifs were selectively targeted. The NL2 CD contains one PPxY and two PxxP motifs (Schema 4) that may bind type I WW and SH3 domains, respectively (reviewed in Kay et al., 2000). An additional Prolinerich stretch beginning 39 residues from the $\mathrm{C}$-terminus is predicted to bind a wide variety of proteins and binding modules including SH3, WW, EVH1 and Profilin (reviewed in Kay et al., 2000; Kato et al., 2004).

These consensus sequences were targeted by site-directed mutagenesis in the NL2 ${ }_{C D}$ bait constructs and examined in the yeast-two-hybrid system. After selective mutagenesis of the NL2 CD bait construct, two mutants had combinatorial effects on all putative interaction partners in yeast (Table 3). Mutant NL2/P768A/ Y770A, targeting the WW domain-binding motif, abolished the interaction of at least 5 candidates, while a P798stop mutation truncating the NL2 CD just upstream of the proline-rich stretch abolished the interaction with more than 10 candidates. The remaining candidates that were to able to interact with both the aforementioned mutants in yeast were not able interact with a combined NL2/ P768A/Y770A/P798stop mutant. This result is indicative of the importance of the proline motifs in the NL2 CT and provides us with NL2 mutants that lose their ability to interact with any of the given putative interaction partners from the yeast-twohybrid screen. 


\begin{tabular}{|c|c|c|c|c|c|c|}
\hline & $\begin{array}{c}\mathrm{NL} 2 / \\
\triangle \mathrm{PDZ}\end{array}$ & $\begin{array}{c}\text { NL2/ } \\
\text { P768A/ } \\
\text { Y770A }\end{array}$ & $\begin{array}{c}\text { NL2/ } \\
\text { P798stop }\end{array}$ & $\begin{array}{c}\text { NL2/ } \\
\text { P768A/ } \\
\text { Y770A/ } \\
\text { P798stop }\end{array}$ & DG & Gephyrin \\
\hline Aida & ++ & ++ & + & - & - & - \\
\hline EB1 & + & + & + & - & - & - \\
\hline DRP2 (long) & ++ & ++ & ++ & - & ++ & - \\
\hline$\beta$-Catenin & ++ & + & + & - & - & - \\
\hline Spinophilin & ++ & + & + & - & - & - \\
\hline S-SCAM (WW) & ++ & $(+)$ & + & - & ++ & - \\
\hline Nedd4-WW2,3 & ++ & + & + & - & + & - \\
\hline Magi-3 & ++ & ++ & $(+)$ & & + & - \\
\hline Endophilin & ++ & ++ & + & & - & - \\
\hline Groucho & ++ & + & - & & - & - \\
\hline Profilin II & ++ & + & - & & - & - \\
\hline ArgBP2 & ++ & ++ & $(+)$ & & - & - \\
\hline POSH & + & + & - & & - & - \\
\hline TOCA-1 & ++ & ++ & - & & - & - \\
\hline Rapostlin & + & + & - & & - & - \\
\hline VASP & ++ & ++ & - & & - & - \\
\hline DAAM1 & ++ & ++ & - & & - & - \\
\hline Fe65 & ++ & ++ & - & & - & - \\
\hline Fe65 like & ++ & ++ & $(+)$ & & - & - \\
\hline GAS7 & ++ & ++ & - & & - & - \\
\hline MAGI-1 & ++ & ++ & - & & - & - \\
\hline S-SCAM (PDZs) & ++ & ++ & - & & . & - \\
\hline$\beta$ IV Spectrin & ++ & - & $(+)$ & & - & - \\
\hline Sca I & + & - & - & & - & - \\
\hline Kalirin & + & - & $(+)$ & & - & - \\
\hline SynGAP & ++ & - & + & & - & - \\
\hline F gephyrin & ++ & - & + & & - & + \\
\hline E gephyrin & ++ & - & + & & - & \\
\hline a-actinin & $(+)$ & - & - & & - & - \\
\hline Abi-1 & $(+)$ & $(+)$ & $(+)$ & & - & - \\
\hline FBP II & $(+)$ & $(+)$ & - & & - & - \\
\hline DRP2 (short) & $(+)$ & - & $(+)$ & & & - \\
\hline \multicolumn{7}{|c|}{ Interaction with both PPxY and poly-Proline } \\
\hline \multicolumn{7}{|c|}{ Interaction dependant on poly-Proline } \\
\hline \multicolumn{7}{|c|}{ Interaction dependant on PPxY } \\
\hline
\end{tabular}

Table 3: Summary of yeast-two-hybrid readouts of candidate NL2 interaction partners against bait constructs of NL2 mutants, Dystroglycan and Gephyrin.

\subsubsection{Investigative Neuroligin 2 protein interaction assays with puta- tive interaction partners}

To examine the validity of putative interactions with NL2, prey fragments from the yeast-two-hybrid screen were subcloned into mammalian expression vectors, in- 
serting an N-terminal enhanced Green Fluorescent Protein (GFP) tag. This allowed the refinement of the screen using cell biological and biochemical methodologies.

\subsubsection{Biochemical detection of Neuroligin 2-prey fragment complexes}

GFP-tagged constructs of selected prey fragments (see Table 4) were transiently transfected into cultured HEK 293FT cells, chosen for their high yields of recombinant protein expression. Protein products were visualized after SDS lysis of the cells, SDS-PAGE and immunoblotting using a monoclonal mouse-anti-GFP antibody. Having determined that the GFP-prey fragment fusion proteins are properly expressed in this preparation, interaction with the NL2 CD could potentially be assessed by standard co-immunoprecipitation and binding assay methodologies in heterologous expression systems.

Table 4: Flowchart of assays performed (+) on NL2 candidate interaction partners.

\begin{tabular}{|c|c|c|c|c|c|}
\hline & $\begin{array}{l}\text { co-immuno- } \\
\text { precipitation }\end{array}$ & $\begin{array}{l}\text { GST-fusion } \\
\text { binding }\end{array}$ & $\begin{array}{c}\text { Fc-fusion } \\
\text { binding }\end{array}$ & co-localization & co-clustering \\
\hline a-actinin & & & & & + \\
\hline Abi-1 & & & & & + \\
\hline Aida & & & & & + \\
\hline \multicolumn{6}{|l|}{ ArgBP2 } \\
\hline$\beta$-IV Spectrin & & & & & + \\
\hline$\beta$-Catenin & & & & + & + \\
\hline \multicolumn{6}{|l|}{ DAAM1 } \\
\hline DRP2 & + & + & + & + & + \\
\hline EB1 & & & & & + \\
\hline \multicolumn{6}{|l|}{ Endophilin } \\
\hline \multicolumn{6}{|l|}{ FBP11 } \\
\hline Fe65 & & & & + & + \\
\hline Fe65-like & & & & & + \\
\hline GAS7 & & & & & + \\
\hline Gephyrin & + & + & + & + & + \\
\hline Groucho & & & & + & + \\
\hline Kalirin & & & & + & + \\
\hline \multicolumn{6}{|l|}{ MAGI-1 } \\
\hline \multicolumn{6}{|l|}{ MAGI-3 } \\
\hline Nedd4 & & & & & + \\
\hline
\end{tabular}




\begin{tabular}{|c|c|c|c|c|c|}
\hline & $\begin{array}{l}\text { co-immuno- } \\
\text { precipitation }\end{array}$ & $\begin{array}{c}\text { GST-fusion } \\
\text { binding }\end{array}$ & $\begin{array}{c}\text { Fc-fusion } \\
\text { binding }\end{array}$ & co-localization & co-clustering \\
\hline $\mathrm{POSH}$ & & & & & + \\
\hline POSH-like & & & & & + \\
\hline Profilin II & & & & + & + \\
\hline \multicolumn{6}{|l|}{ Rapostlin } \\
\hline \multicolumn{6}{|l|}{ Sca I } \\
\hline Spinophilin & + & & & + & + \\
\hline S-SCAM & & & & + & + \\
\hline SynGAP & & & & + & + \\
\hline TOCA-1 & & & & + & + \\
\hline VASP & & & + & + & + \\
\hline S-SCAM (WW) & & & & + & + \\
\hline E-Gephyrin & + & & & + & + \\
\hline DRP2-short & + & & + & + & + \\
\hline
\end{tabular}

For co-immunoprecipitation assays, cultured HEK 293FT cells were transiently cotransfected with selected GFP-tagged prey fragment constructs (Table 4) along with an N-terminally-tagged full-length NL2 expression construct (HA-NL2) or one of the respective constructs which correspond to the mutation which in yeast abolished interaction with a given prey (Table 2). TritonX-100 extracts were examined for co-expression of GFP and HA tagged proteins using SDS-PAGE and immunoblotting with monoclonal mouse-anti-GFP and monoclonal mouse-anti-HA antibodies. After co-expression was determined, extracts were incubated with monoclonal mouse-anti-HA antibody. Antibody-bound proteins and protein complexes were immunopurified using Protein G-conjugated Sepharose beads which bind immunoglobulins, and analyzed by SDS-PAGE and immunoblotting. HAtagged proteins were enriched in the immunopurification eluate, though none of the GFP fusion proteins were specifically enriched in the HA-NL2 unmutated samples. myc-S-SCAM, a tagged version of a known PDZ interactor of Neuroligins (Hirao et al., 1998), was found to co-precipitate (Figure 2), exemplifying the capacity of this assay to detect HA-NL2-containing complexes. Lack of specific coimmunoprecipitation of GFP-prey fusion proteins persisted after examining a range of extraction conditions in terms of ionic strength (from 50 to $500 \mathrm{mM} \mathrm{NaCl}$ ), the presence of $\mathrm{Ca}^{2+}$ and detergent (Nonidet P-40, Cholate, and n-Octyl- $\beta$-Dglucoside) used for membrane protein extraction. This indicates either that the se- 


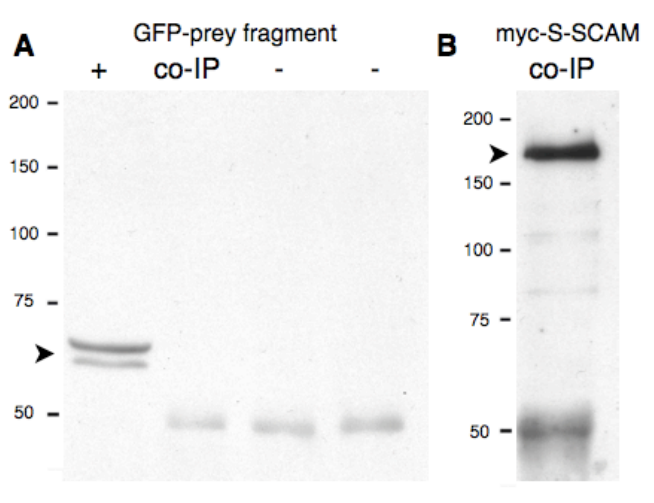

Figure 2: Co-immunoprecipitation of NL2 with candidate interaction partners. (A) Example immunoprecipitation of HA-NL2 with interaction candidate DRP2 (short) from the yeast-two-hybrid screen. Extracts from HEK 293FT cells co-transfected with GFP-DRP2s HA-NL2 (lanes 1-2), HA-NL2 binding deficient mutant (lane 3 ) or empty vector (lane 4) were co-immunoprecipitated with anti-HA antibodies. Extract (lane 1) and precipitate (lane 2-4) samples were immunoblotted for GFP. (B) Myc immunoblot of HA immunoprecipitate in extracts of HEK 293FT cells co-expressing myc-S-SCAM and HA-NL2. Molecular weight markers are indicated in $\mathrm{KDa}$. Arrowheads indicate the anticipated GFP- or myc-tagged protein bands. The immunoglobulin heavy chain band is visible in the precipitate lanes migrating at $50 \mathrm{KDa}$.

lected candidates do not form complexes with NL2 in the heterologous system, or that the extraction conditions disrupt complexes present in cells.

To avoid the use of detergent extraction for the isolation of protein complexes, interaction assays using soluble proteins can be employed in binding assays in solution. To perform these experiments, the CD of NL2 wild-type, NL2/P768A/ Y770A, NL2/P798stop and NL2/P768A/Y770A/P798stop sequences were subcloned into bacterial expression vectors which add an N-terminal Glutathione Stransferase (GST) tag. GST fusion constructs were expressed in E. coli from which protein extracts were subjected to affinity purification using glutathione-conjugated Sepharose beads. Purified proteins were eluted from the beads and visualized by Coomassie blue staining after SDS-PAGE. All fusion constructs appeared to be expressed, however their electrophoretic mobility indicated that the vast majority of purified protein consisted of truncated fusion protein variants, most of which corresponded to GST alone. Only a small fraction of the purified material represented fusion proteins of the expected electrophoretic mobility (Figure $3 \mathrm{~A}$ ), which may be due to the potentially natively unfolded nature of the Neuroligin CD, making it susceptible to degradation in bacteria. This expression problem was not overcome by standard methods of inhibiting proteolysis and ultimately confounded GST-fusion binding assays due to very low signal-to-noise ratios of the readout.

To sidestep the problem of bacterial expression of NL2 CD constructs in E. coli, their respective sequences were subcloned into mammalian expression constructs which generate cytosolic fusion proteins where the C-terminus contains the human immunoglobulin heavy chain (Fc). HEK 293FT cells were transiently transfected 


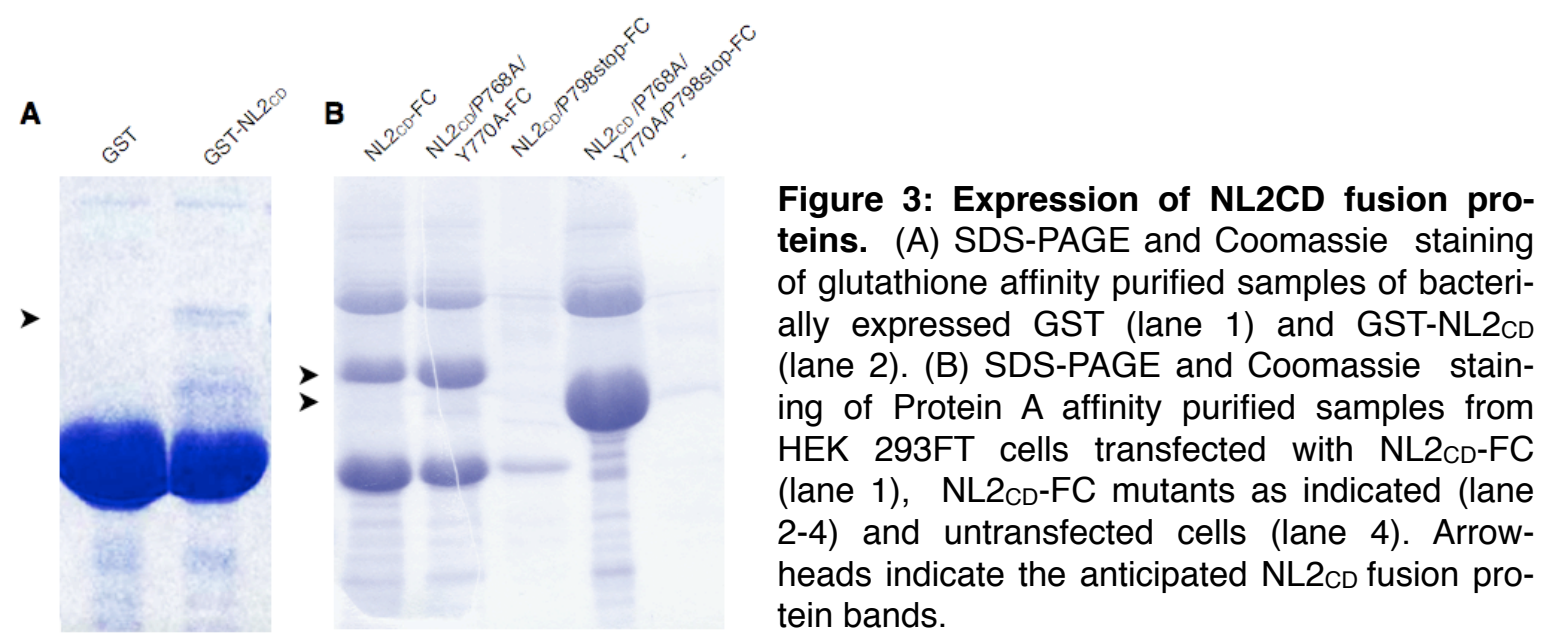

with NL2CD-Fc constructs and fusion proteins were purified from homogenate supernatants of the cells using protein A-conjugated Sepharose beads. Expression and purity of the proteins was examined by SDS-PAGE and Coomassie staining of total proteins (Figure $3 \mathrm{~B}$ ) or immunoblotting with a goat-anti-human IgG1. Soluble $\mathrm{NL2}$ CD-Fc fusion proteins were expressed and purified without the problems encountered with the bacterially expressed GST fusion proteins.

NL2 $\mathrm{CD}-\mathrm{Fc}$ fusion proteins immobilized on protein A-conjugated Sepharose beads were used to perform pulldown experiments on selected prey fragment fusion proteins (Table 3). GFP-prey fragment constructs were used to transfect HEK 293FT cells and homogenate supernatants of the cells were used as input for Fc fusion binding assays. Homogenates were incubated with NL2 $\mathrm{CD}-\mathrm{Fc}$ coated beads or the respective mutants shown to abolish interaction in yeast as negative controls. Several ionic strength conditions (ranging from 50 to $500 \mathrm{mM} \mathrm{NaCl}$ ) were examined, however readouts for all candidates and all conditions examined showed no specific pulldown of GFP-fusion proteins by NL2CD-Fc.

\subsubsection{Co-expression of Neuroligin 2 vs. prey fragments in heterologous cells}

Complementary approaches to biochemical isolation of protein complexes in studying protein interactions can be sought in cell biological methodologies. For proteins to be able to interact in a cellular context, they must localize to a common subcellular compartment. Fluorescent immunodetection of two proteins in cellular preparations can be examined for colocalizing signals indicating that the two proteins encounter each other in the cell. Using the inherent fluorescent properties of 
the GFP-tagged prey fragments and immunodetecting the HA tag of HA-NL2 constructs, colocalization may be assayed in a co-expressing heterologous cell system. The GFP fusion proteins are expected to be soluble and thus localize to cytoplasmic compartments, while HA-NL2 is a transmembrane protein and thus must localize to plasma membrane and/or intracellular membranous structures. For HANL2 to interact with one of the GFP-fused candidates, the latter must be present at juxtamembrane positions.

COS7 cells were selected due to their morphological characteristics of having extended regions where the plasma membrane is flat and easy to image. GFPtagged constructs of selected prey fragments (Table 3) along with HA-NL2 or its respective mutant constructs were transiently co-transfected into cultured COS7 cells. Inherent GFP fluorescence and fluorescence immunodetection of the HA tag after fixation and permeabilization were used to simultaneously visualize exogenous protein distribution in individual co-transfected cells 12 to 24 hours posttransfection.

In virtually all cases, HA-NL2 immunoreactivity was prominently detected diffusely on the plasma membrane and in perinuclear regions - presumably corresponding to compartments of the secretory pathway. GFP fluorescence adopted varying distribution paterns dependent on the prey fragment component of the fusion protein examined. Notwithstanding the anticipated heterogeneity, several general features of GFP-fusion protein distribution could be observed throughout the selection of candidates. Two patterns of GFP fluorescence were most prominent; diffuse distribution throughout the cytosol and in aggregate form at cytoplasmic accumulations of varying morphology. Nuclear fluorescence was also prominent in many samples - a potential artifact known to arise from the GFP tag. Each fusion protein distributed between these patterns differently, covering a wide spectrum between candidates that had virtually undetectable diffuse distribution and abundant cytosolic aggregates, like DRP2 and Gephyrin, and candidates which exhibited aggregated GFP fluorescence only sporadically, like Profilin II.

In order to closely compare the subcellular distributions of GFP fusion proteins and HA-NL2, the respective fluorescent images were overlaid and colocalization manifesting in overlapping fluorescent signals was assessed. In all samples HA immu- 

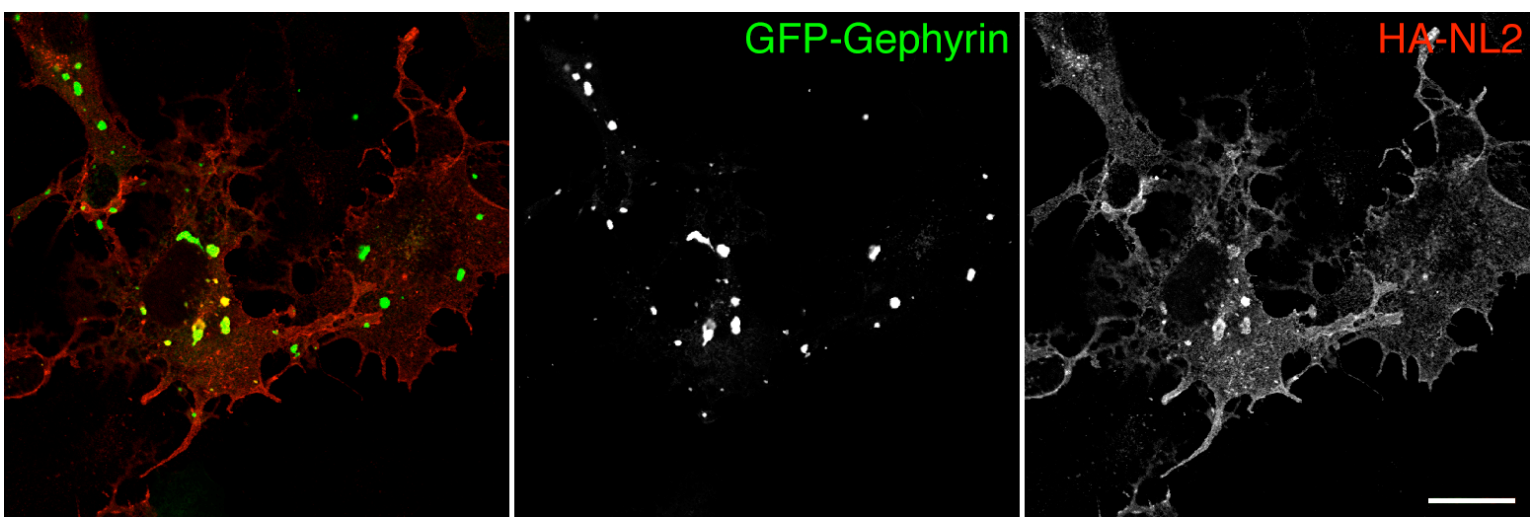

Figure 4: NL2 sporadically colocalizes with Gephyrin aggregates in heterologous cells. HEK 293FT cells were cotransfected with GFP-Gephyrin and HA-NL2. HA-NL2 (red) immunoreactivity showed mainly perinuclear and plasma membrane distribution patterns, while GFPGephyrin (green) was overwhelmingly sequestered in distinct large cytoplasmic aggregates. In less than $20 \%$ of the cells, HA-NL2 immunoreactivity was localized along with GFP-Gephyrin in aggregates. Scale bar: $20 \mu \mathrm{m}$.

noreactivity was absent from aggregated GFP structures with the possible exception of GFP-gephyrin where HA immunoreactivity appeared sequestered in GFPGephyrin cytoplasmic aggregates sporadically in less than $20 \%$ of co-transfected cells examined (Figure 4; see § 3.2.4). Perinuclear HA immunoreactivity was also uncorrelated with GFP fluorescence in all samples examined, indicating that NL2 does not associate with candidate proteins during intracellular transport. At the plasma membrane however, GFP fluorescence often appeared enriched to varying extents depending on the GFP-fusion protein examined. The most extensive colocalization at the plasma membrane was observed on occasion with the short fragment fusion protein of DRP2 (Figure 5). Together these observations indicate that HA-NL2 and many of the fusion proteins can meet at the same cellular com-
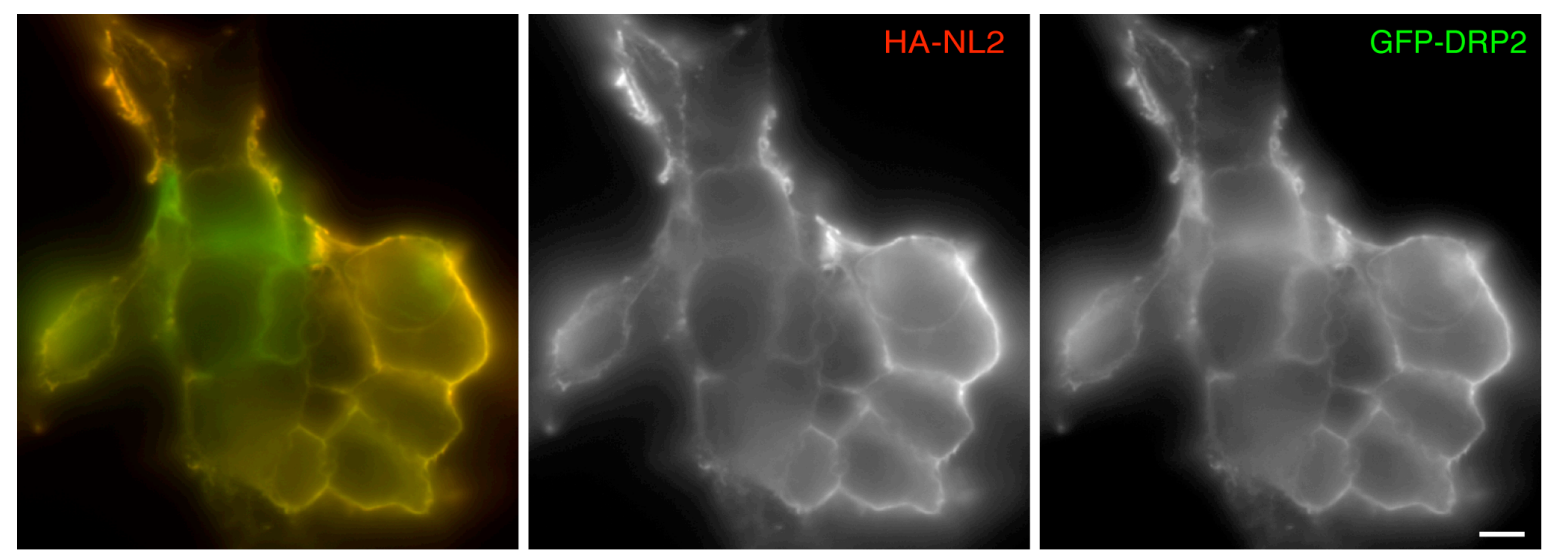

Figure 5: DRP2 colocalizes with NL2 in heterologous cells. HEK 293FT cells were cotransfected with GFP-fusion prey fragment DRP2s (residues 289-659; green) and HA-NL2 (red). Both proteins exhibited extensively overlapping distribution patterns at the plasma membrane. Scale bar: $10 \mu \mathrm{m}$. 
partment, namely the plasma membrane, thus allowing for interaction in the context of the cell.

\subsubsection{Cell biological evidence of Neuroligin 2-prey fragment complexes}

Potential interaction of HA-NL2 and a given fusion protein at the level of the plasma membrane could lead to translocation of a GFP-fusion protein to the plasma membrane in the presence of HA-NL2. An observable shift in GFP distribution to the juxtamembrane compartment in cells co-expressing HA-NL2 could signify such an interaction. This recruitment event would also be indicative of a mechanism that may be relevant in synaptic recruitment events during synaptogenesis in vivo, the identification of which was the ultimate aim of the screening process.

GFP fluorescence distribution was studied in sets of co-transfected COS7 cells where each GFP-fusion protein was co-expressed with HA-NL2 or with the respective mutant which abolished interaction for that specific prey fragment in yeast and served as a negative control. GFP distribution was compared in the two preparations and specific cortical enrichment of GFP fluorescence was examined versus the negative control. In all cases where GFP cortical enrichment was observed, it appeared in both preparations, indicating that an endogenous cellular component was recruiting GFP-fusion proteins to juxtamembrane sites.

Many of the interaction candidates are known regulators of the actin cytoskeleton (see Table 1), and bind either directly or indirectly to actin. Mammalian cells are known to have a rich plexus of F-actin at the level of the plasma membrane termed cortical actin, which may provide the substrate for the juxtamembrane recruitment of many of the GFP-fusion proteins. Cells co-transfected as previously described were additionally stained with fluorophore-conjugated phalloidin, a compound which binds F-actin thereby labeling the actin cytoskeleton. Triple labeling revealed that GFP cortical enrichment, along with local enrichments in the plasma membrane distribution of HA-NL2, followed the intensity pattern of cortical actin (data not shown). This indicates that cortical actin organizes the juxtamembrane distribution of several GFP fusion proteins. Interestingly it also raises the possibility that HA-NL2 itself is associated with the actin cytoskeleton. Additional support 

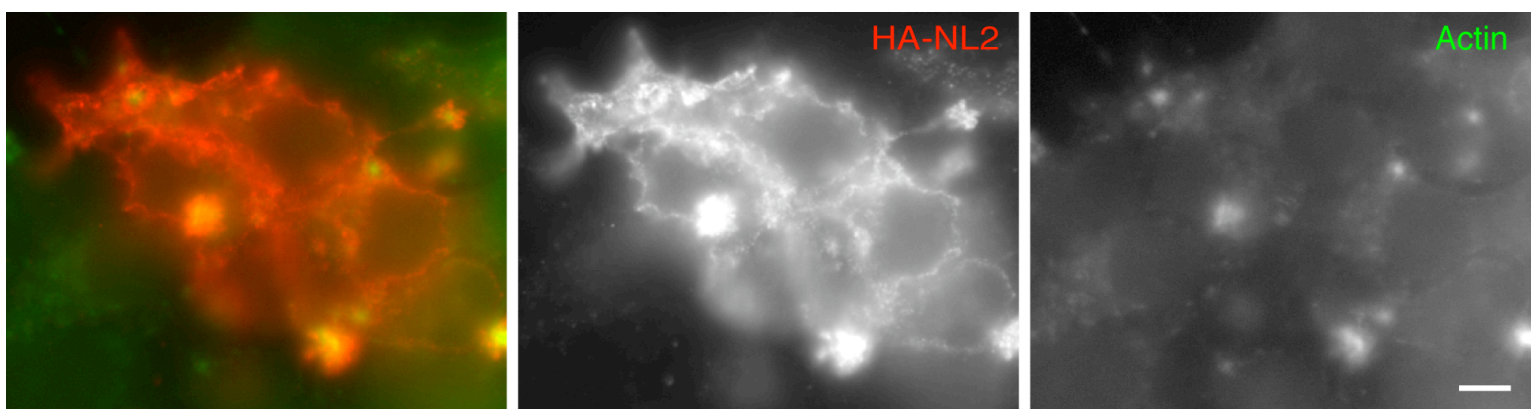

Figure 6: NL2 associates with actin structures in heterologous cells. HEK 293FT cells were transfected with HA-NL2, treated with Latrunculine A and labeled for HA (red) and actin (green). Treatment caused a pronounced redistribution of actin to cytoplasmic globular structures where HA-NL2 was consistently enriched.

for this notion comes from observations of HA-NL2 sequestration in G-actin paracrystals induced by treatment with the toxin Latrunculine A (Figure 6), and the induction of filopodia-like protrusions upon overexpression of HA-NL2 in hippocampal neurons in culture (data not shown).

To overcome potential masking of specific HA-NL2-mediated membrane recruitment by cortical actin association of GFP fusion proteins, a recruitment assay was devised which could discriminate between the two. The basis of this cell biological assay is the manipulation of HA-NL2 on the plasma membrane to induce a patterned distribution which can be distinguished from that of cortical actin or diffuse juxtamembrane enrichment. This assay was performed as a screening procedure on GFP-fusion constructs corresponding to 22 of the 30 yeast-two-hybrid candidate interaction partners of NL2 (see Table 3).

COS7 cells co-transfected with a given GFP-fusion protein and HA-NL2 or its respective mutants as negative controls were treated with monoclonal mouse-antiHA antibody and subsequently fluorescently-conjugated goat-anti-mouse antibodies in situ, just prior to fixation. This treatment induced surface clustering of HAtagged membrane proteins, sequestering them into discrete plasma membrane

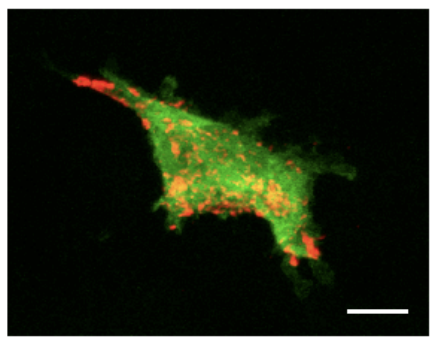

Figure 7: NL2 clustering assay. Projection of 3D reconstitution from serial optic slices showing an example of a COS7 cell co-transfected with HA-NL2 and a GFP-fusion prey construct after two-step antibody clustering of HA. Scale bar represents $10 \mu \mathrm{m}$. 

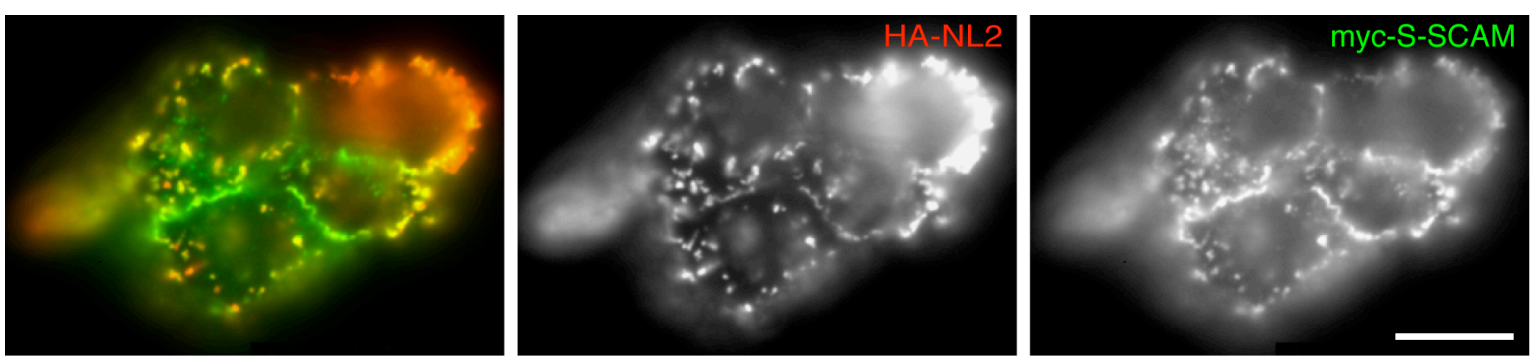

Figure 8: NL2 co-clusters with S-SCAM in heterologous cells. HEK $293 \mathrm{FT}$ cells cotransfected with HA-NL2 (red) and myc-S-SCAM (green). The two proteins showed extensive coclustering after two-step antibody clustering of HA. Scale bar represents $10 \mu \mathrm{m}$.

domains (Figure 7). HA-NL2 surface clusters were able to recruit myc-S-SCAM (Figure 8), providing a positive control for the ability of these clusters to recruit interacting cytosolic proteins.

GFP fluorescence was examined in these preparations for enrichment under the HA surface clusters. From the candidates examined (Table 3), GFP-DRP2(short) exhibited clear recruitment to HA-NL2 surface clusters (Figure 9), but not to clusters of binding-deficient HA-NL2/P768A/Y770A/P798stop mutant, indicating that DRP2 can interact with NL2 and be recruited to the plasma membrane in heterologous cells. While the recruitment was striking when observed, it occurred only in a minority of co-transfected cells and did not appear to correlate with expression levels, potentially indicating that the interaction may be inducible or dependent on a transient state of the cell. In the same assay, massive recruitment to HA-NL2 surface clusters was observed with GFP-Gephyrin, however this occurred only upon co-expression of a co-factor (see Chapter 3.2.4).

Taken together, cell biological and biochemical assays on fusion proteins corresponding to the yeast-two-hybrid candidate interaction partners of NL2 did not yield conclusive evidence confirming interaction for any one candidate. In the cell biological assays DRP2 appeared as the most promising candidate showing positive, albeit circumstantial, results. In an attempt at obtaining more concrete evidence from experiments involving the endogenous protein, polyclonal antibodies were designed against specific epitopes of rodent DRP2. The obtained antisera exhibited specific affinity for recombinant DRP2 as expressed in heterologous cell versus non-expressing cells. However, an immunoreactive band with the anticipated electrophoretic mobility was not observed in brain homogenates from adult 

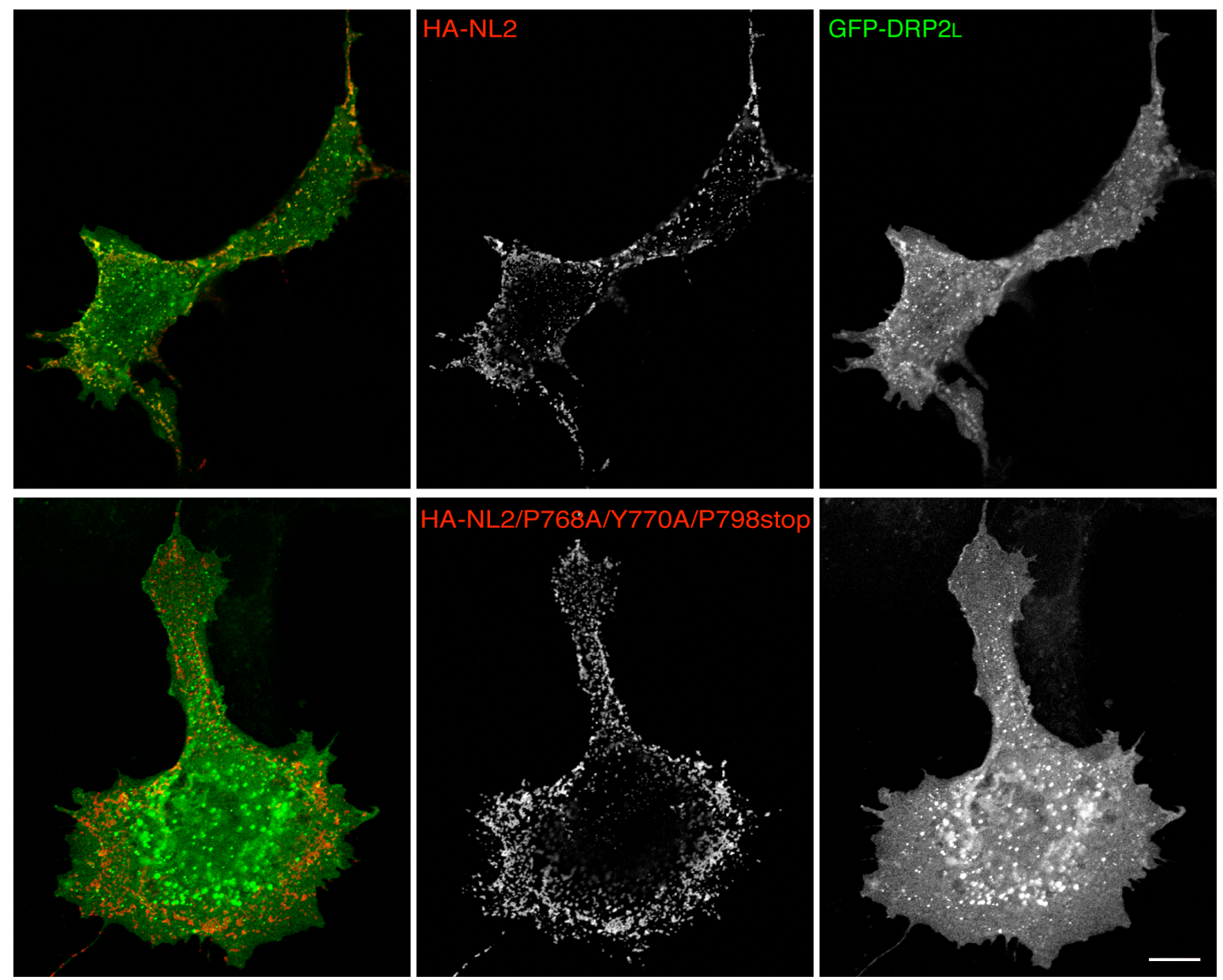

Figure 9: NL2 sporadically co-clusters DRP2 in heterologous cells. COS7 cells were cotransfected with HA-NL2 (red) and GFP-fusion prey fragment DRP2L (residues 89-828; green). Some cells exhibited significant co-clustering of the two proteins after two-step antibody clustering of HA, while others showed no recruitment of GFP fluorescence to plasma membrane sites (top row of panels). Co-clustering was never observed when mutant HA-NL2/P768A/Y770A/ P798stop was clustered (bottom row of panels). Scale bar represents $10 \mu \mathrm{m}$.

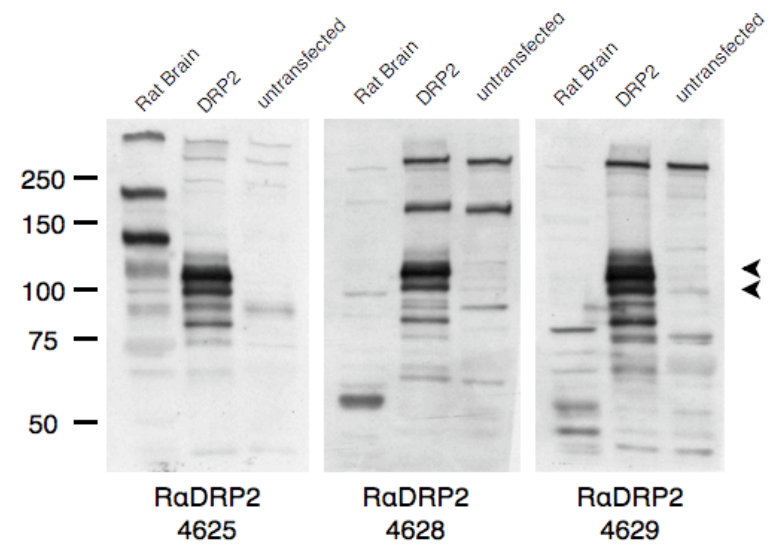

Figure 10: Specificity of DRP2 antibodies. Immunoblots using three rabbit-antiDRP2 antisera.. immunoblotting was performed on rat brain homogenate (left lane $\checkmark$ in each panel), DRP2-transfected HEK 293FT cells (middle lanes in each panel) and untransfected HEK 293FT cells (right lanes in each panel). Arrowheads indicate the anticipated range of migration of DRP2 bands.

rat (Figure 10). It thus remains to be seen whether DRP2 is expressed in the CNS and whether it may be associated with NL2 or have a functional role at inhibitory synapses. 


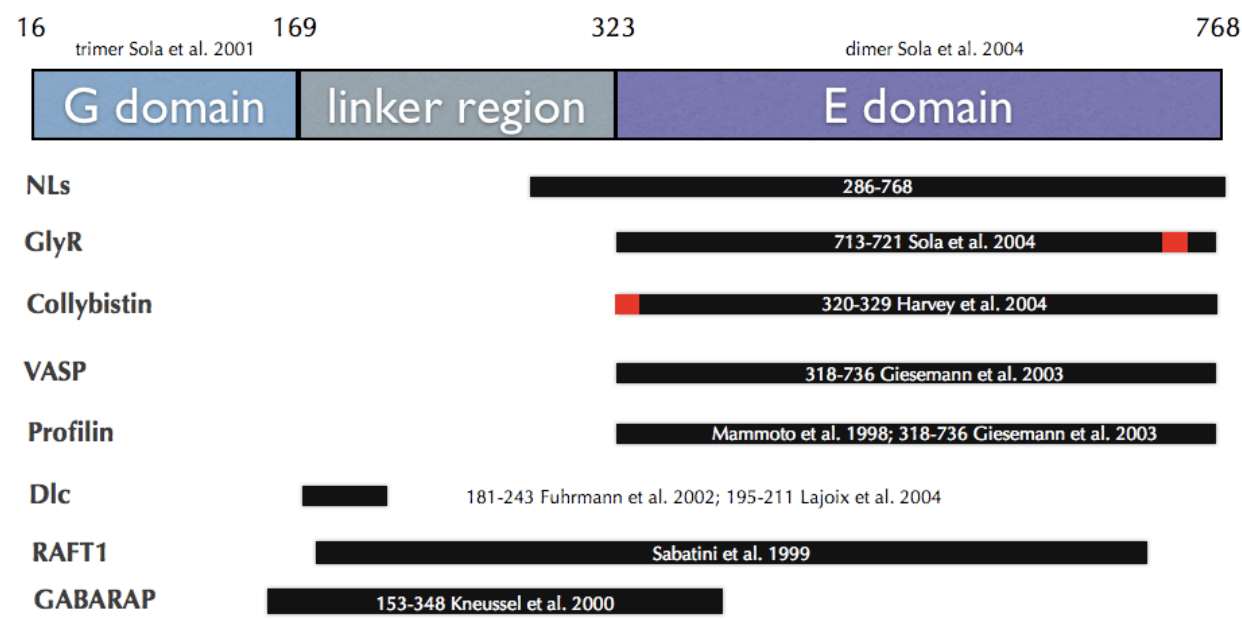

Schema 6: Gephyrin domain structure and interaction partners. Gephyrin is divided into three regions: the G-domain, linker region and E-domain. The residue numbers shown correspond to the P1 variant of Gephyrin. Previously identified Gephyrin interaction partners are listed and the Gephyrin regions sufficient to bind them are displayed in reference to the gephyrin domain schematic with black bars enclosing residue numbers. Regions in red represent sequence stretches necessary for binding with the respective interaction partner. The region sufficient for Neuroligin binding is also shown.

\subsection{Study on the role of Neuroligin 2 in the assembly of the inhibitory postsynaptic scaffold}

Investigative biochemical and cell biological analyses of potential cytosolic interaction partners of NL2 did not produce substantial evidence to conclusively identify interaction partners. They were however encouraging observations for two of the candidates, namely DRP2 and Gephyrin. As Gephyrin is the core scaffolding protein of inhibitory synapses, data from these pilot experiments prompted closer examination of its potential interaction with NL2.

\subsubsection{Gephyrin is a putative cytosolic binding partner of Neuroligins}

Two independent clones for Gephyrin were identified in the yeast-two-hybrid screen, corresponding to the full-length protein and a C-terminal fragment (residues 286-768) starting just upstream of the Gephyrin E-domain (Schema 6). Surprisingly, Gephyrin prey constructs yielded equally strong signals with NL1, NL3 and NL4 full-length cytosolic bait constructs (Figure $11 \mathrm{~A}$ ), potentially signifying that Gephyrin, like PDZ domain proteins of excitatory postsynapses, is a core Neuroligin interaction partner. 

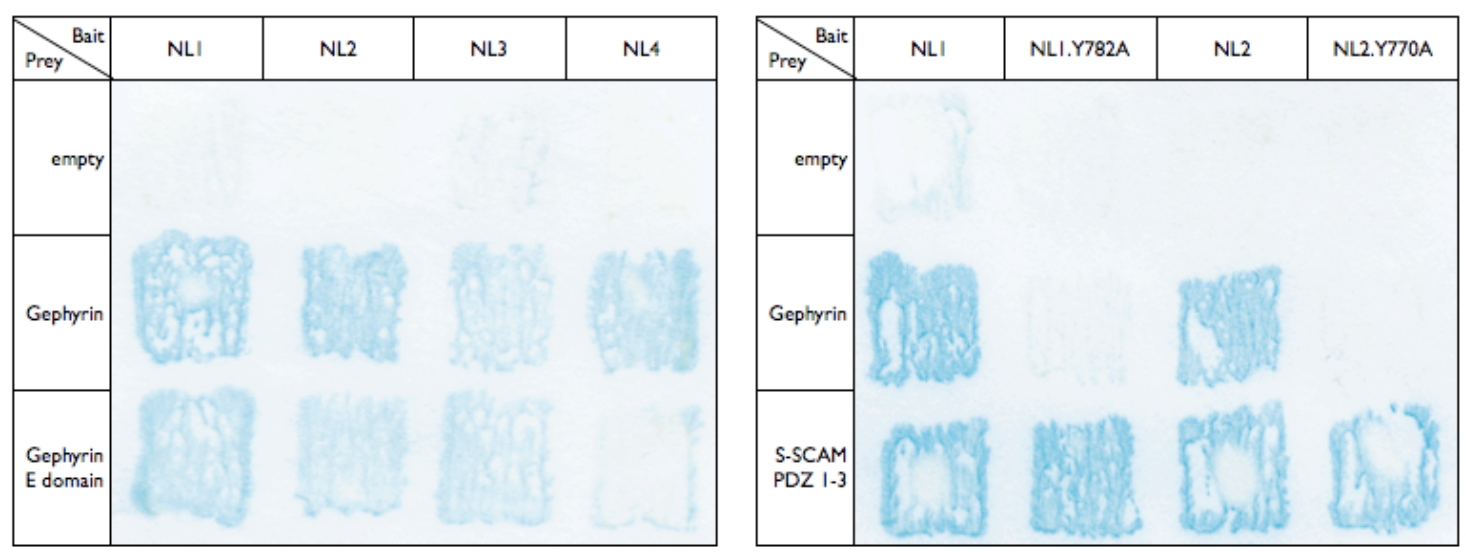

Figure 11: Neuroligins interact with Gephyrin in yeast. (A) LacZ reporter-gene readouts of yeast-two-hybrid assays with Neuroligin full cytoplasmic domain bait against Gephyrin full-length (middle row) and E-domain (bottom row) prey clones that were isolated as candidate cytoplasmic interaction partners of NL2 in the yeast-two-hybrid screen. (B) LacZ reporter-gene readouts of yeast-two-hybrid assays with NL1 and NL2 full cytoplasmic domain bait constructs and their respective NL1/Y782A and NL2/Y770A mutants against prey constructs of full-length Gephyrin and an S-SCAM fragment encompassing its three PDZ domains (residues 422-976). Readouts of empty prey vector against the Neuroligin bait constructs is shown in the first rows as an indicator of LacZ background levels due to autoactivity.

In the analysis of binding-deficient NL2 mutants in yeast, the putative interaction with Gephyrin was abolished by the NL2/P768A/Y770A mutation targeting putative WW domain-mediated interactions. As Gephyrin does not exhibit sequences homologous to WW domains, the significance of the NL2 mutation was further examined. Mutation of the full-length CD of NL2 residue 770 from tyrosine to alanine alone abolished interaction with Gephyrin as did the double point mutant P768A/ Y770A. However, mutation of proline residue 768 to alanine alone did not affect the interaction with Gephyrin in yeast (Figure 12). This indicates that the putative interaction of NL2 with Gephyrin is dependent on NL2 tyrosine 770 but not on the integrity of the NL2 PPXY WW-binding consensus motif.

The critical NL2 residue for interaction with Gephyrin is conserved in the other three Neuroligin paralogues (highlighted in Schema 4). Mutation of the homolo-

bait:

NL2/Y770A

NL2

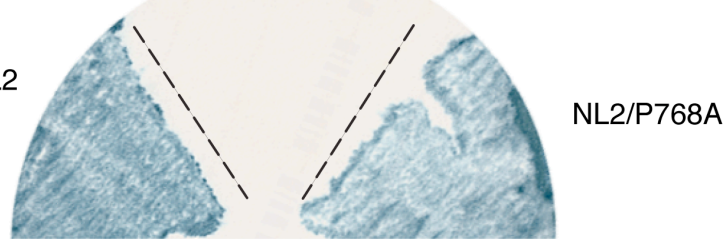

prey: Gephyrin
Figure 12: Selected mutations abolish NL2 binding to Gephyrin in yeast. LacZ reporter-gene readouts of yeasttwo-hybrid assays with the cytoplasmic domain of wild-type NL2, NL2/Y770A and NL2/P768A bait constructs against a full-length Gephyrin prey construct. The Y770A mutation abolishes the NL2Gephyrin interaction while the P768A mutation has no effect. 
gous residue Y782 in NL1 to alanine resulted in loss of Gephyrin interaction (Figure $11 \mathrm{~B})$. Both NL1/Y782A and NL2/Y770A constructs gave positive readouts with a PDZ domain-containing S-SCAM prey fragment (Figure $11 \mathrm{~B}$ ) and with prey fragments of other putative interactors (data not shown), confirming that the constructs are expressed and functional in yeast. This indicates that NL1 and 2 interact with Gephyrin via the same mechanism. Altogether, these experiments show that a Y770-containing epitope and the C-terminal PDZ-binding motif (highlighted in Schema 4) independently mediate binding of NL2 to Gephyrin and PDZ-domain proteins, respectively.

\subsubsection{Neuroligin 2 and Gephyrin form a complex in brain and heter- ologous cells}

In brain sections and neurons in culture endogenous NL2 is immunodetected in clusters along with Gephyrin at GABAergic and glycinergic postsynaptic membranes. To examine whether NL2 can be in physical complex with Gephyrin, biochemical isolation of a protein complex containing both NL2 and Gephyrin-derived proteins was attempted.

Heterologous co-expression of HA-NL2 with GFP-Gephyrin or GFP-E-Gephyrin in COS7 cells was employed to examine whether the two proteins coimmunoprecipitate from non-ionic detergent extracts. While various detergent and buffer conditions were assayed, none led to co-precipitation of GFP constructs in immunoprecipitates of anti-HA antibodies.

As detergents can interfere with protein complex integrity, the necessity of using detergents to extract transmembrane proteins was bypassed by replacing full length HA-NL2 with tagged soluble fragments of the NL2 CD in co-precipitation experiments. GFP-NL2CD was co-expressed with myc-Gephyrin and cell homogenates devoid of detergent were subjected to immunoprecipitation with antiGFP antibodies. Equivalently, an alternative construct was used where the human immunoglobulin heavy chain was fused C-terminally to the NL2 CD sequence (NL2 $\mathrm{CD}-\mathrm{FC})$. This construct was directly precipitated from cell homogenates coexpressing GFP-Gephyrin or GFP-E-Gephyrin using Protein A conjugated Sepharose beads. In all the above cases, no co-precipitation was observed. 
Though the above classical protein complex detection assays yielded negative results, it is noteworthy to state that soluble NL2 CD constructs lose their ability to associate with heterologous Gephyrin membrane aggregates, a property of fulllength HA-NL2 (see § 3.2.4). As both, the former detergent-extracted preparations and the latter soluble protein homogenate preparations take the potential NL2Gephyrin complex out of its native context of the plasma membrane, it remains a possibility that this complex may robustly manifest itself only in the juxtamembrane milieu. Indeed, though GST-fusion binding assays with GST-NL2CD were hampered by poor full-length fusion protein expression (see $\S 3.1 .2 .1$ ), the use of recombinant purified Gephyrin allowed the detection of Gephyrin in complex with GST-NL2 $2 \mathrm{CD}$ and not with the predicted binding-deficient mutant GST-NL22D/Y770A (Ingo Paarman, Frankfurt, personal communication). Gephyrin present in the in vitro protein complex was observed only slightly above the detection limit of the methodology, indicating that the interaction in solution may have low affinity or transient properties.

In order to obtain biochemical evidence for a NL2-Gephyrin complex, a preparation was selected where complexes are stabilized in their native environment prior to extraction based on an approach previously reported to detect postsynaptc membrane protein complexes (Leonard et al., 1998). Crude synaptosomal preparations from mouse brains were chemically crosslinked by acute treatment with the membrane permeable, homobifunctional crosslinker DSP. After crosslinking, total proteins were extracted with SDS. This treatment is known to extract membrane proteins and disrupt protein interactions in the PSD, and is thus expected to release endogenous NL2 in solution coupled only with proteins covalently crosslinked during treatment of synaptosomes. Extracts of crosslinked synaptosomes, containing $1 \%$ SDS, were diluted with 7 volumes of $1 \%$ TritonX-100 solution. A 1:7 detergent ratio of SDS to TritonX-100 produces a mixed micelle lysate which does not denature antibodies or interfere with antigen recognition thus allowing further analysis with immunological techniques.

Immunoprecipitation using a polyclonal antibody against endogenous NL2 (Varoqueaux et al., 2004) was carried out on the crosslinked preparations and immunoprecipitates were subjected to crosslinker cleavage and subsequent analysis by SDS-PAGE. Endogenous Gephyrin was detected in the NL2-containing 
A
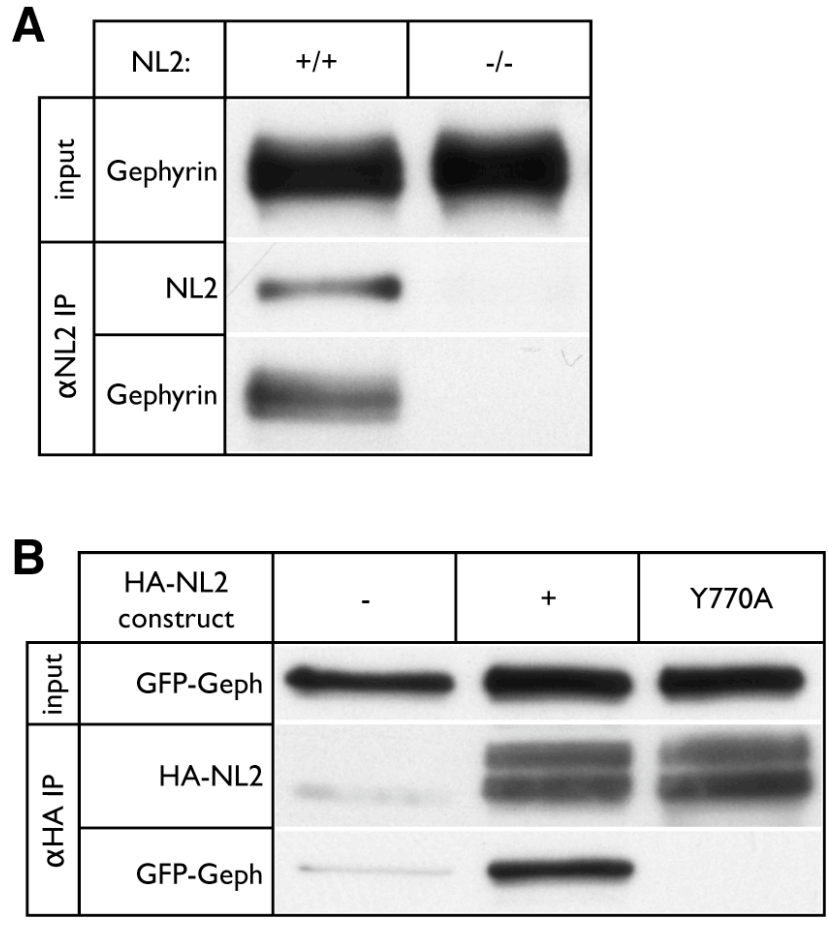

C
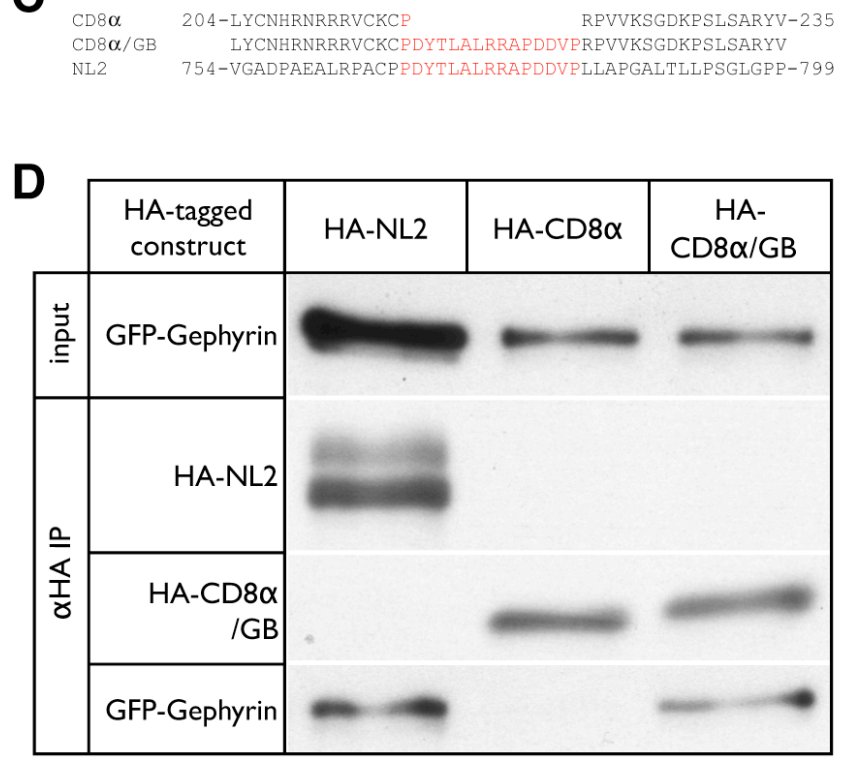

Figure 13: NL2-Gephyrin complexes in brain and heterologous cells. Brain homogenates $(A)$ or live transfected COS7 cells ( $B$ and $D$ ) were chemically crosslinked with DSP and proteins were extracted with SDS. Crosslinked adducts were immunoprecipitated for NL2 and immunoblotted for Gephyrin. (A) Brain homogenate preparations from NL2 wild-type $(+/+)$ or NL2 deletion-mutant mice (-l-) were used. Immunoprecipitation was performed with an NL2-specific antibody. After linker cleavage, Western blot analysis for NL2 (center row) and Gephyrin (top and bottom rows) was performed on input extract (top row, $0.37 \%$ of total loaded) and immunoprecipitated material (center and bottom rows, $30 \%$ of total loaded). (B) COS7 cells were transfected with GFP-Gephyrin along with either empty vector (left lane), HA-NL2 (center lane) or HA-NL2/Y770A (right lane). After treatment of the cells, immunoprecipitation with anti-HA antibody, and linker cleavage, Western blot analysis for HA and GFP tags was performed on samples from input extract (top row, $0.15 \%$ of total loaded) and immunoprecipitated material (center and bottom rows, $30 \%$ of total loaded). (C) Alignment of the cytoplasmic domain sequences of the chimeric HA-CD8a/GB, the HA-CD8a and the HA-NL2 constructs. The GB motif sequence is in red. (D) COS7 cells were transfected with GFP-Gephyrin along with either HA-NL2 (left lane), HA-CD8a (center lane) or HA-CD8a/GB (right lane). After treatment of the cells, immunoprecipitation with anti-HA antibody, and linker cleavage, Western blot analysis for HA and GFP tags was performed on samples from input extract (top row, $0.37 \%$ of total loaded) and immunoprecipitated material (center and bottom rows, $30 \%$ of total loaded).

crosslinked adducts in preparations from wild-type mice but not from equivalent preparations from NL2 deletion mutant mice (NL2-/-) (Figure $13 \mathrm{~A}$ ). This indicates that non-specific precipitation of Gephyrin does not reach the detection threshold of the methodology, and that co-precipitated Gephyrin is part of a crosslinked protein complex containing NL2.

To examine whether NL2 and Gephyrin form a complex without other synaptic proteins mediating an indirect link as protein liaisons, COS7 cells were transfected with GFP-Gephyrin alone or with GFP-Gephyrin and HA-NL2 or HA-NL2/Y770A. 
Live double transfected cells were subjected to in situ chemical crosslinking, and proteins were extracted with SDS and immunoprecipitated for HA. GFP-Gephyrin was identified as a specific component of crosslinked adducts containing HA-NL2 but not the Gephyrin-binding-deficient mutant HA-NL2/Y770A (Figure 13 B). The fact that NL2-Gephyrin complexes were isolated from non-neuronal cells speaks against the possibility of synaptic proteins indirectly connecting the two proteins; however the possibility of ubiquitous protein components acting as adaptors cannot be excluded. However, the fact that the complex is abolished by the Y770A mutation that in yeast abolished NL2 $\mathrm{CD}$ interaction with Gephyrin shows that the NL2-Gephyrin complexes we detected are a product of an interaction structurally equivalent to the direct interaction observed in yeast.

\subsubsection{Identification of a novel Gephyrin-binding motif conserved in Neuroligins}

In view of the yeast-two-hybrid and biochemical data showing that all Neuroligin paralogues can interact with Gephyrin and that this interaction depends on a conserved tyrosine residue, it is likely that a putative Gephyrin-binding motif on the Neuroligin CD would encompass the tyrosine crucial for binding and would be conserved in all four Neuroligins. A 15-residue stretch in the Neuroligin CD, where Y770 is at position 3, fits these criteria (Schema 4 and Figure $13 \mathrm{C}$ ). To examine whether these 15 residues can incur Gephyrin-binding properties on a transmembrane protein, this sequence was inserted into the CD of a HA-tagged expression construct of an unrelated transmembrane protein, namely human CD8 $\alpha$ (HACD8 $\alpha / G B$; Figure $13 \mathrm{C}$ ). The ability of this chimera to bind Gephyrin was examined using the same crosslinking approach in COS7 cells. While GFP-Gephyrin was not detected in HA-CD8 $\alpha$ purified crosslinked adducts, it was detected crosslinked with $\mathrm{HA}-\mathrm{CD} 8 \alpha / \mathrm{GB}$, as it was with HA-NL2 (Figure $13 \mathrm{D}$ ). This indicates that the conserved 15-residue stretch within the Neuroligin CD is sufficient to convey Gephyrin-binding properties to transmembrane proteins. It is therefore proposed that this peptide sequence of the Neuroligin protein family represents a novel Gephyrin-binding motif. 
A NL1 DYTLAMRRSPDDVP NL2 DYTLALRRAPDDVP NL3 DYTLTLRRSPDDIP NL4 DYALTLRRSPDDVP

B

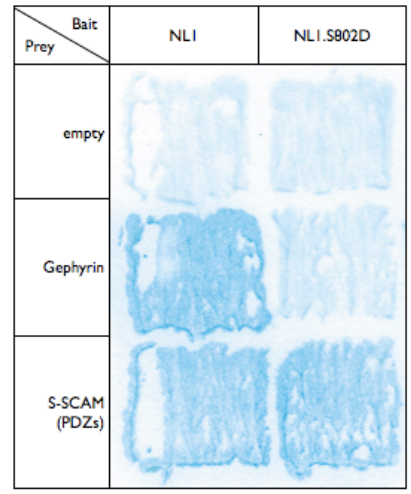

Figure 14: The Neuroligin Gephyrin-binding motif. (A) Alignment of the Gephyrin-binding motifs of rodent Neuroligins 1 through 4 . Serine and threonine residues are in red. (B) LacZ reportergene readouts of yeast-two-hybrid assays of bait $\mathrm{NL} 1 \mathrm{CD}$ and phosphomimetic mutant S802D against empty prey vector, full-length Gephyrin and S-SCAM fragment (422-976, encompassing PDZ domains 1-3) prey.

The Neuroligin Gephyrin-binding motif shares $73 \%$ identity and $87 \%$ homology between the four rodent paralogues. The conserved nature of this motif is disrupted only in three positions, namely 4, 6 and 10, in which serine or threonine residues are substituted with alanines in different paralogues (Figure $14 \mathrm{~A}$ ). As these residues are classical targets of kinases, it is conceivable that phosphorylation may differentially regulate Gephyrin binding to the Neuroligin paralogues. The serine at position 10 presents special interest as it is predicted to be phosphorylated by PKA or other serine/threonine kinases primarily due to the two upstream arginine ne (Songyang et al., 1994). Importantly, this site has an alanine substitution only in $\mathrm{NL2}$, the paralogue that has consistently been observed associated with Gephyrin. Phosphomimetic substitution S802D of NL1 at this serine position led to the abolishment of the interaction with Gephyrin in yeast-two-hybrid assays (Figure $14 \mathrm{~B}$ ). This indicates that potential phosphoserine at position 10 of the Neuroligin Gephyrin-binding motif may negatively regulate interaction with Gephyrin.

\subsubsection{Neuroligins are recruited to Collybistin-induced Gephyrin mem- brane aggregates in heterologous cells}

To study the cellular correlate of the NL-Gephyrin interaction in mammalian cells, tagged Neuroligin constructs were expressed in COS7 cells. As previously described, Neuroligins were visible diffusely across the plasma membrane and in the perinuclear region. When cotransfected with GFP-Gephyrin, NL2 distribution remained essentially unchanged, with the exception of sporadic (in less than $20 \%$ of cotransfected cells) recruitment to large cytoplasmic aggregates (Figure 4), which 
are typically observed upon Gephyrin expression (Kirsch et al., 1995). It seemed plausible that Gephyrin within these aggregates might not be accessible to Neuroligins on the plasma membrane. The Gephyrin-binding protein Collybistin is known to be involved it the translocation of cytosolic Gephyrin to plasma membrane sites (Kins et al., 1999; Harvey et al., 2004; Papadopoulos et al., 2007). A splice variant
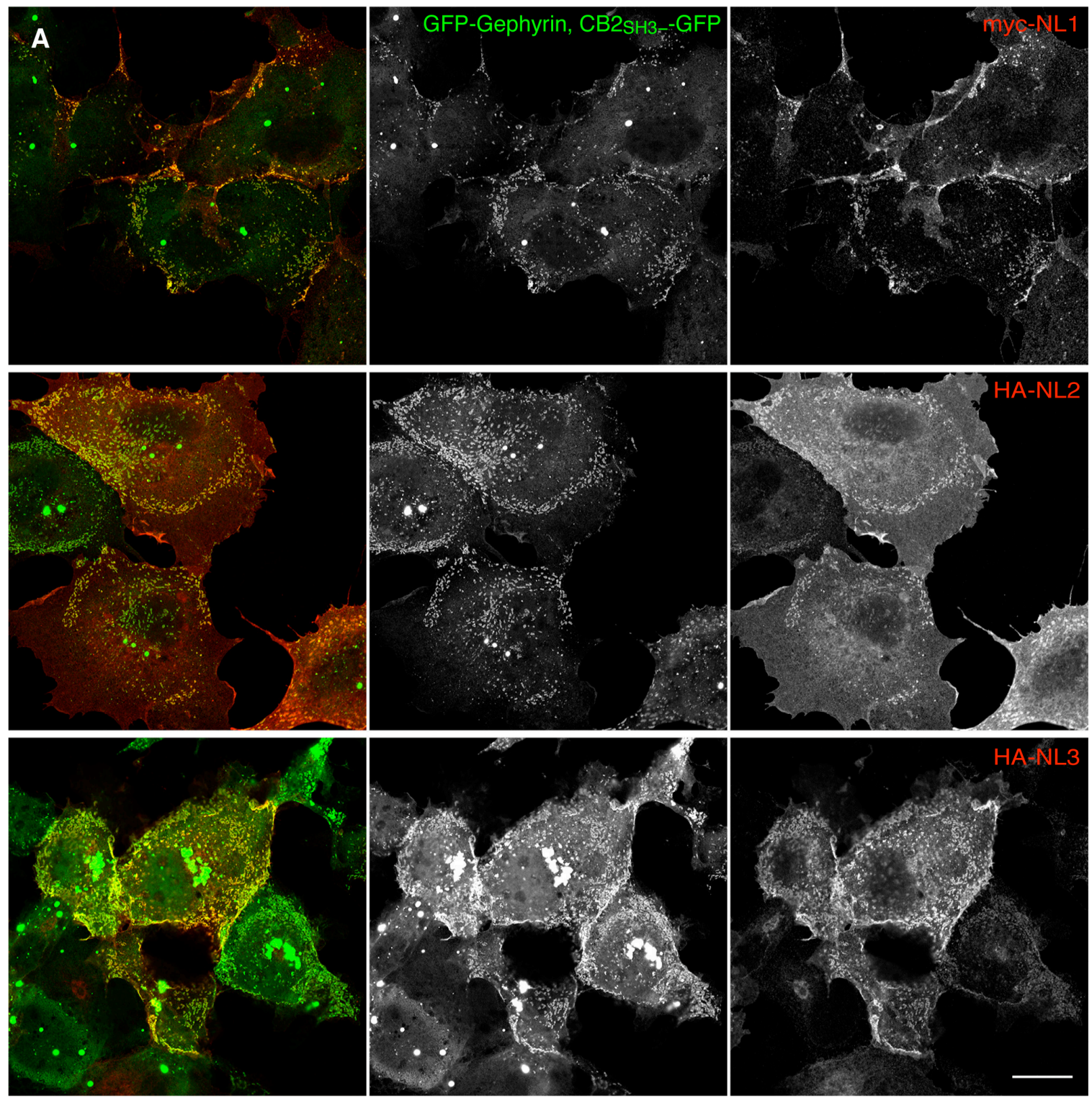

B

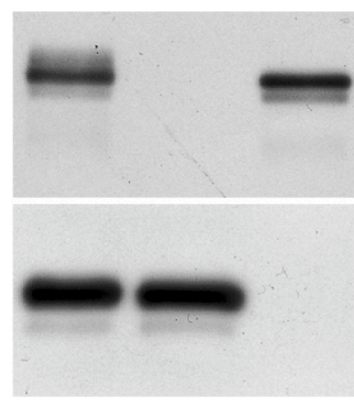

Figure 15: Neuroligins are enriched at plasma membrane sites of Gephyrin-Collybistin microaggregates in heterologous cells. (A) COS7 cells were co-transfected with GFP-Gephyrin (green), CB2sH3-GFP (green) and tagged NL constructs (red). Tagged NL1, 2 and 3 spontaneously cocluster at Gephyrin-Collybistin membrane microaggregates. Scale bar: $20 \mu \mathrm{m}$. (B) Immunoblots of COS7 cell lysates expressing GFP-Gephyrin alone (right lane), CB2sH3--GFP alone (center lane) and the two together (left lane). Note that the presence of $\mathrm{CB}_{2 \mathrm{SH}} \mathrm{S}_{-}$ GFP influences the electrophoretic mobility pattern of GFP-Gephyrin. 
lacking the Collybistin $\mathrm{SH} 3$ domain (CB2 $\left.\mathrm{SH}_{-}\right)$induces the association of Gephyrin with the plasma membrane in microaggregate form when co-transfected into mammalian cells (Kins et al., 1999). Thus, introduction of CB2sH3- into cells coexpressing NL2 and Gephyrin would bring these proteins in the same cellular compartment, namely the plasma membrane.

COS7 cells were co-transfected with expression constructs of either myc-NL1, HANL2 or HA-NL3, and GFP-Gephyrin along with CB2sH3-GFP. Strikingly, upon triple transfection Neuroligins massively redistributed to GFP-positive membrane microaggregates (Figure $15 \mathrm{~A}$ ). The same was observed using an $\mathrm{N}$-terminally myctagged Gephyrin construct derived from the full-length yeast prey clone and Cterminally GFP-tagged E-Gephyrin, corresponding to the short yeast prey fragment (data not shown). These data indicate that Collybistin has a role in promoting the association of Gephyrin and Neuroligins.

To examine whether the direct Neuroligin-Gephyrin interaction identified is involved in their coclustering at the plasma membrane, the overlapping pattern of GFP clusters, corresponding to both Gephyrin and Collybistin, with full-length HANL2, HA-NL2/Y770A or FLAG-NX1a as an unrelated transmembrane protein control was compared in COS7 cells (Figure $16 \mathrm{~A}$ ). Correlation analysis of the respective fluorescence intensities showed that HA-NL2 correlated significantly more with GFP-containing structures than its Gephyrin-binding mutant $(p<0.001)$, which did not significantly differ from background correlation determined by FLAG-NX1a with GFP (Figure 16 B). A residual enrichment of HA-NL2/Y770A with GFP could occasionally be observed (Figure $16 \mathrm{~A}$ ), indicating that this mutant may retain some affinity for Gephyrin or that secondary cellular mechanisms may also be involved. It is noteworthy that cotransfection of HA-NL2 and CB2sH3--GFP alone, as such or followed by surface clustering of HA, did not result in enrichment of GFP fluorescence at sites of plasma membrane HA clusters (data not shown). This finding is not compatible with a direct binding of $\mathrm{CB}_{2 \mathrm{SH} 3}$ - to NL2, and an adaptor role of $\mathrm{CB}_{2 \mathrm{SH}}$ - in the NL2-Gephyrin interaction. Taken together, these data indicate that Neuroligins are specifically recruited to Gephyrin clusters formed upon Collybistin expression in heterologous cells, via direct interaction with Gephyrin. 


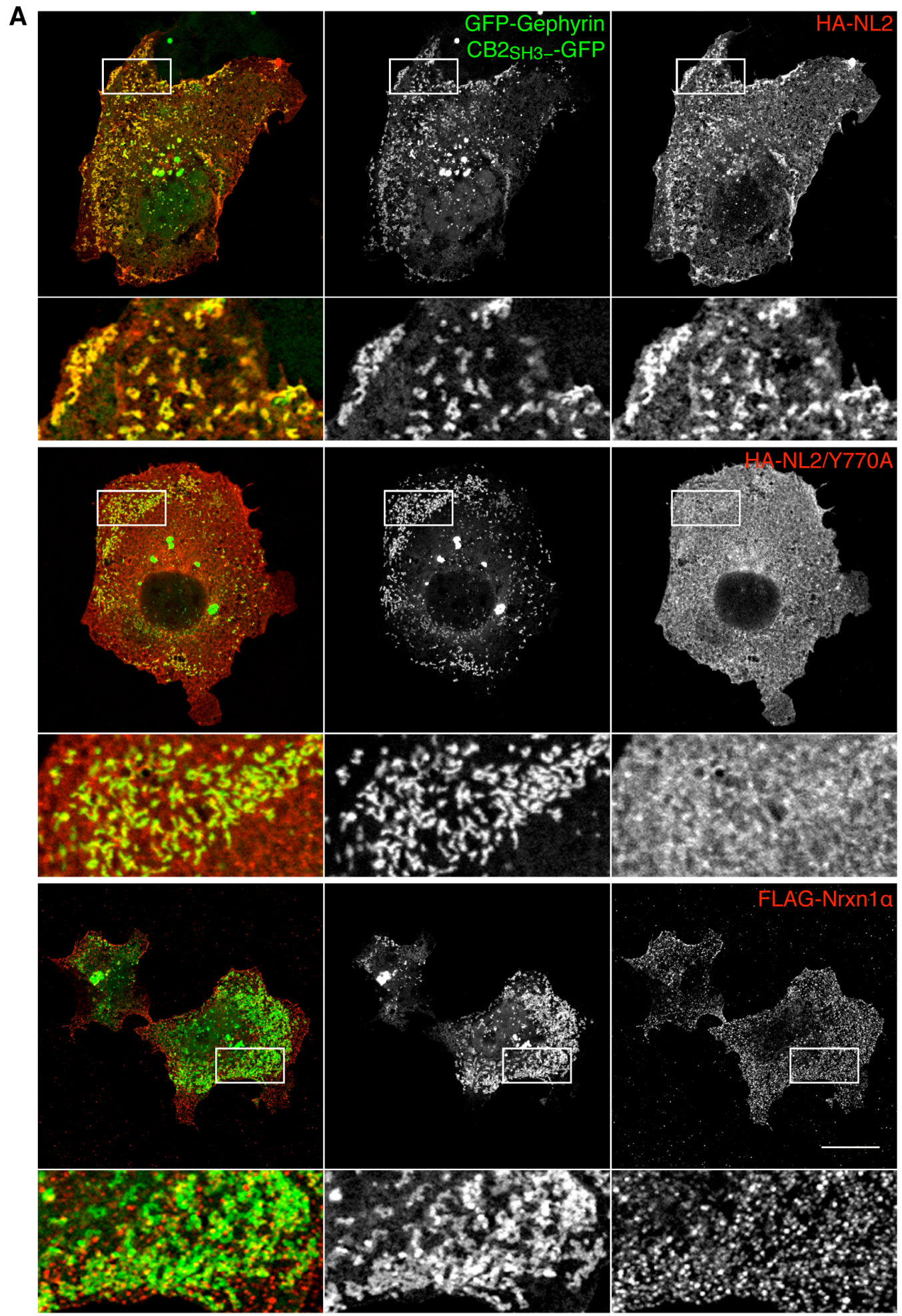

B

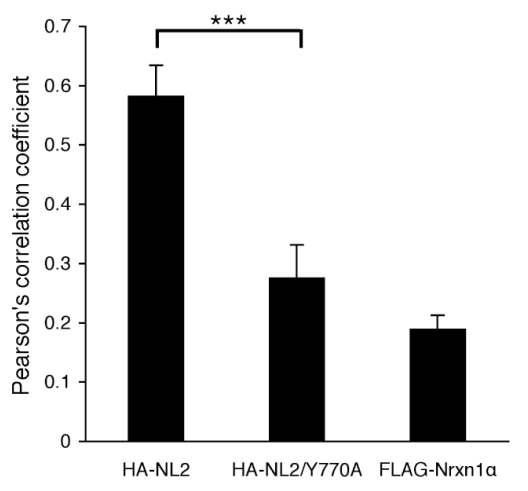

Figure 16: NL2 enrichment at Gephyrin-Collybistin membrane microaggregates via NL2-Gephyrin Interaction. (A) COS7 cells were cotransfected with GFPGephyrin (green), CB2sH3-GFP (green) and either HA-NL2, HA-NL2/Y770A or control FLAG-NX1a constructs (red). Scale bar: $20 \mu \mathrm{m}$, main panels; $4.6 \mu \mathrm{m}$, insets. (B) Intensity correlation analysis of green vs. red immunoreactivity. GFP fluorescence correlates significantly more with HA-NL2 $(0.584 \pm 0.051, \mathrm{n}=10 ; * * *, \mathrm{p}<0.001)$ than with HA-NL2/ Y770A $(0.277 \pm 0.055, n=11)$. The latter does not significantly correlate with GFP fluorescences more than the negative control FLAG-NX1a does $(0.19 \pm 0.023, n=11)$. 
Collybistin appears to act as a permissive factor for the interaction of Neuroligins with Gephyrin. Its role may be through altering the compartmentalization of gephyrin or via its proposed properties as a signaling molecule. Collybistin has been proposed to function as a specific Cdc42 GDP/GTP exchange factor (GEF), promoting the activation of signaling cascades downstream of Cdc42. To examine whether such signaling cascades may be involved in promoting NeuroliginGephyrin association, pilot experiments were performed using Cdc42 mutants which manipulate its activation status in transfected cells (reviewed by Bollag \& McCormick, 1991). A constitutively active mutant of Cdc42 (HA-Cdc42/Q61L) was co-transfected along with GFP-Gephyrin and HA-NL2, replacing Collybistin used in the previous experiments. In these samples, the distributions of Gephyrin, mostly in cytoplasmic aggregates, and NL2, mostly at the plasma membrane, appeared unaffected, indicating that Cdc42 activation alone was not sufficient to induce NL2Gephyrin co-clustering.

To examine whether Cdc42 signaling is a component part of the Collybistin mechanism, a dominant negative mutant of Cdc42 (HA-Cdc42/T17N) was cotransfected along with CB2sH3-GFP, HA-NL2 and GFP-Gephyrin. Again, the presence of the Cdc42 mutant had no effect on the anticipated distribution of the other proteins, which persisted to co-cluster at the plasma membrane (data not shown), indicating that Cdc42 activation is not necessary for co-clustering to take place. These data speak against a role for Cdc42 signaling in the function of Collybistin to promote Neuroligin-Gephyrin association. However, an unidentified signaling role of Collybistin may still be implicated. A hint for such a possibility came from

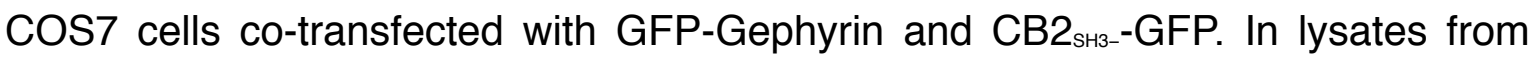
co-expressing cells, the elecrophoretic mobility of Gephyrin was altered showing an additional slower-migrating diffuse minor band. This band was absent from cells expressing Gephyrin alone, indicating that post-translational modification of Gephyrin may be induced in the presence of $\mathrm{CB}_{\text {sнз- }}$ (Figure $15 \mathrm{~B}$ ). Taken together, these observations indicate that $\mathrm{CB}_{\text {знз- }}$ promotes Neuroligin-Gephyrin coclustering in a manner that is independent of its involvement in Cdc42 signaling. Its effect in translocating Gephyrin to the plasma membrane is a plausible mechanism for promoting association by bringing the proteins to the same subcellular 


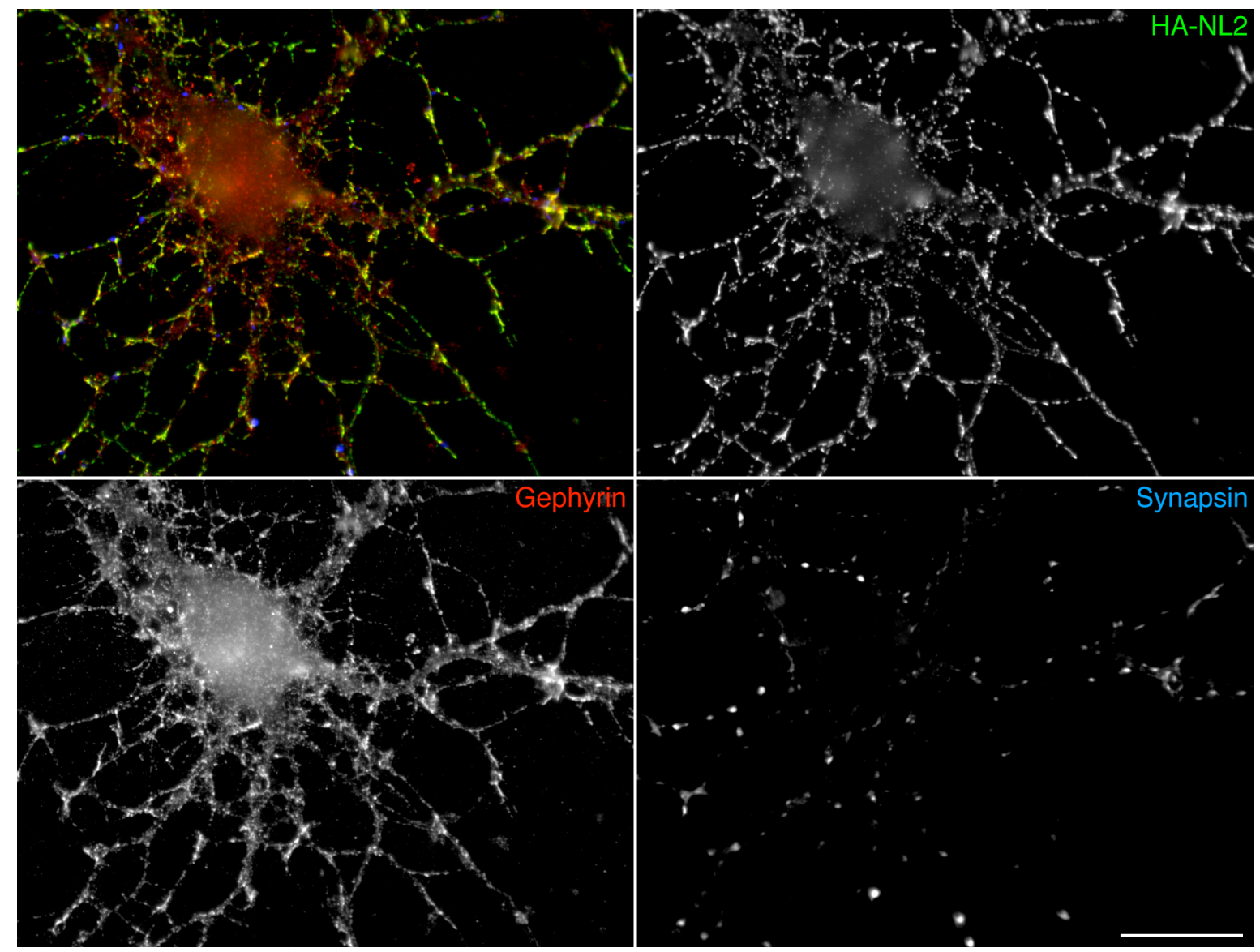

Figure 17: HA surface clustering on rat hippocampal neurons. Hippocampal rat neurons were transfected with HA-NL2 at DIV7. Cells were acutely treated with two-step HA clustering (green), and subsequently labeled for endogenous Gephyrin (red) and Synapsin 1/2 (blue). Acute anti-HA antibody treatment induced the recruitment of endogenous Gephyrin at sites of acutely induced HA-NL2 extrasynaptic clusters. Scale bar: $20 \mu \mathrm{m}$.

compartment, though the involvement of yet unidentified signaling roles of Collybistin cannot be excluded.

\subsubsection{The Neuroligin 2-Gephyrin interaction contributes to their co- clustering in neurons}

In neurons, surface clustering of exogenous NL2 by antibody-coated beads induces Gephyrin recruitment (Graf et al., 2004). To examine whether the direct interaction of NL2 with Gephyrin contributes to this coclustering in neurons, an equivalent approach was employed using an assay which, omitting beads, led to a more consistent readout and allowed for greater sample sizes. Rat neurons overexpressing extracellularly HA-tagged NL2 or CD8a constructs were acutely treated with anti-HA and secondary antibody prior to fixation. Overexpressed HA-NL2 was 
abundantly distributed throughout the plasma membrane of untreated neurons (data not shown). After antibody treatment, HA-NL2 immunoreactivity was redistributed into discrete synaptic and extrasynaptic surface clusters where endogenous Gephyrin was consistently coclustered (Figure 17).

Unexpectedly, all HA-NL2 constructs used in this assay, including the NL2/P768A/ Y770A/P798stop mutant that failed to interact with any of the binding candidates isolated in yeast-two-hybrid screens, showed robust Gephyrin co-clustering (data not shown). This observation was reminiscent of previous studies where Neuroligins carrying loss-of-function mutations in their $\mathrm{C}$-terminus retained much of their normal attributes when introduced into wild-type neurons (Conroy et al., 2007; Dresbach et al., 2004). It thus seemed plausible that extracellular domainmediated oligomerization, which occurs in vitro (Dean et al., 2003; Comoletti et al., 2006; Comoletti et al., 2007) and in neurons (see § 3.3.1 and § 3.3.2), of tagged mutant Neuroligins with endogenous wild-type Neuroligins might have masked loss-of-function effects in these assays.

To circumvent this caveat, constructs were overexpressed in cultured hippocampal neurons from NL2 deletion mutant mice (Figure $18 \mathrm{~A}$ ). In this system, Gephyrin coclustering was generally less robust, potentially as a direct result of the genotype (see $\S$ 3.2.7). However, significant differences between the constructs assayed were now apparent (Figure $18 \mathrm{~A}$ and $\mathrm{B}$ ). In order to quantify the ability of the HAtagged constructs to recruit endogenous Gephyrin, synaptic HA clusters were excluded from the analysis, as endogenous synaptic components could independently recruit and cluster both HA-NL2 and Gephyrin.

Extrasynaptic HA clusters were individually designated using a standardized algorithm based on intensity thresholding and criteria of size and circularity from all transfected neurons identified. As readout for Gephyrin recruitment capacity, the fluorescence intensity ratio of Gephyrin to HA immunoreactivity was measured for each cluster. Quantification demonstrated that HA-NL2 was able to significantly cluster more Gephyrin than HA-NL2/Y770A. Additionally, the HA-CD8 $\alpha / G B$ construct containing the Gephyrin-binding motif of Neuroligins acquired the ability to co-cluster Gephyrin significantly more than HA-CD8 $\alpha$ (Figure $18 \mathrm{~B}$ ). These two observations are in line with the notion of Gephyrin recruitment via the Gephyrin- 
A
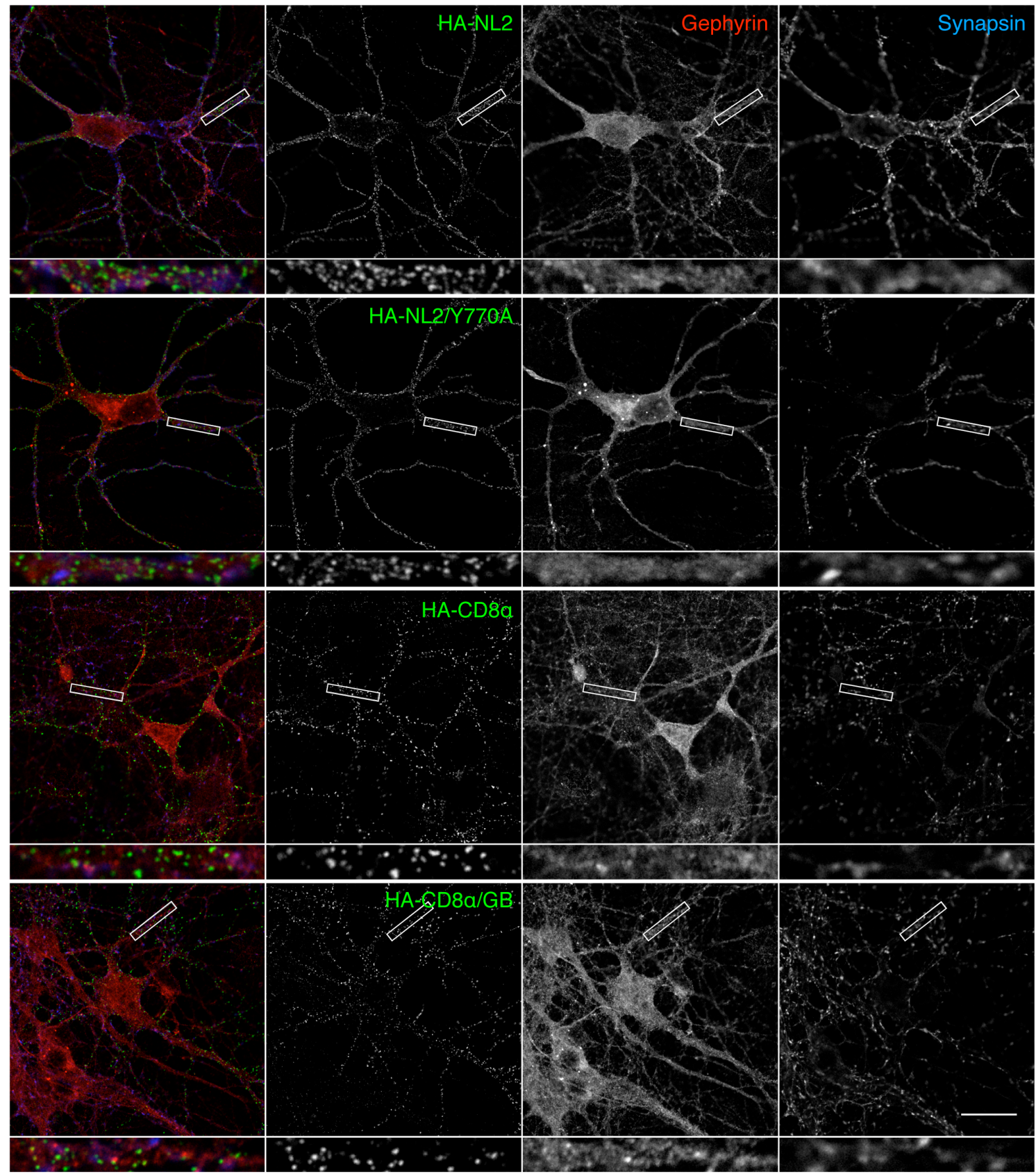

B

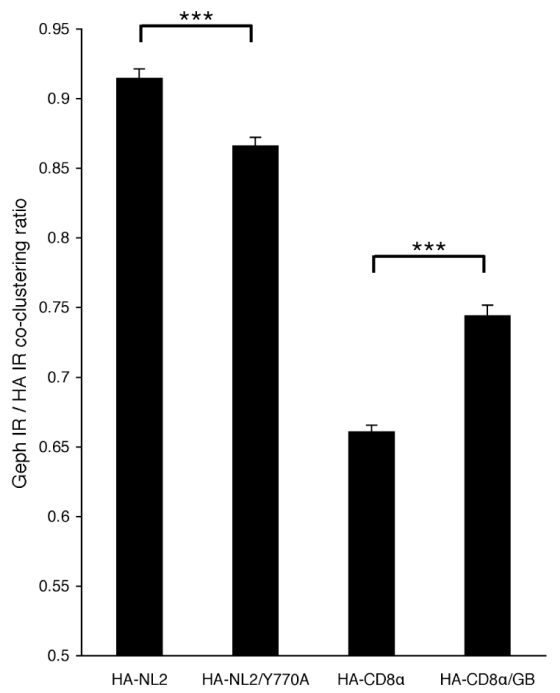

Figure 18: Gephyrin is recruited to sites of NL2 clustering upon direct interaction in cultured neurons. (A) NL2deficient primary hippocampal neurons were transfected at DIV7 with extracellularly HA-tagged membrane protein constructs. These proteins were acutely clustered at the surface upon treatment of the culture with fluorescence-conjugated antibodies (green) at DIV8. Neurons were fixed and stained for endogenous Gephyrin (red) and Synapsin 1/2 (blue). Scale bar: $20 \mu \mathrm{m}$, main panel; 1,64 $\mu \mathrm{m}$, insets. (B) Extrasynaptic HA surface clusters were sorted and quantified for the ratio of overlapping endogenous Gephyrin immunoreactivity vs. HA immunoreactivity. HA-NL2: 0,9152 \pm 0,0064, 29 neurons, $n=4690$ clusters; HA-NL2/Y770A: 0,8664 \pm 0,006, 31 neurons, $n=5712$ clusters; HA-CD8a: 0,6612 $\pm 0,0045,23$ neurons, $n=4830$ clusters; HA-CD8a/GB: 0,7446 $\pm 0,0071$, 12 neurons, $n=2224$ clusters. HA-NL2/Y770A recruits Gephyrin significantly less efficiently than HA-NL2 ( $* * *$, $\mathrm{p}<0,0001$ ) while HA-CD8a/GB recruits Gephyrin significantly more efficiently than HA-CD8a $(p<0,0001)$ in neurons. 
binding domain. A direct comparison between NL2 and CD8 $\alpha$ constructs is not possible due to differences in the expression levels of the constructs and in the number of synaptic contacts they induce. However, if one were to make the comparison, overlooking these liabilities, the data speak of a partial effect indicating the involvement of additional recruitment mechanisms. Nonetheless, together these findings demonstrate that the direct interaction of Gephyrin with NL2 is involved in its recruitment to NL2 clusters in neurons.

\section{A}
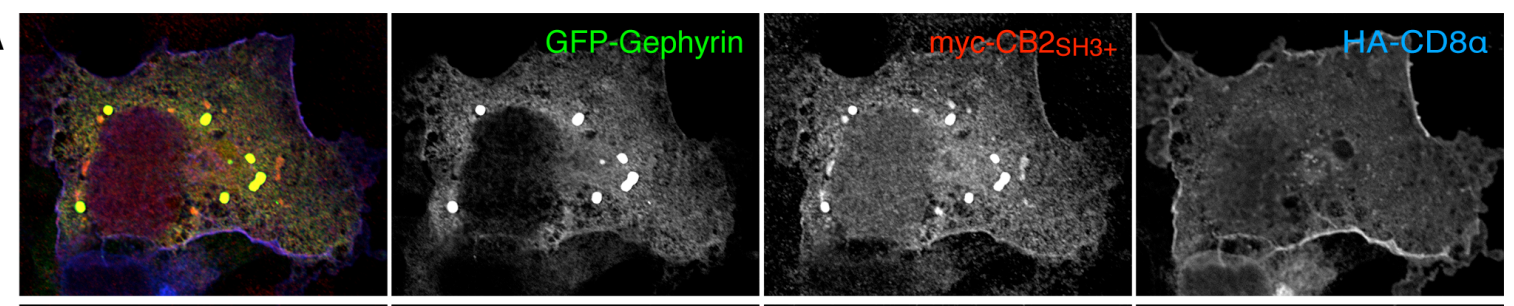

B
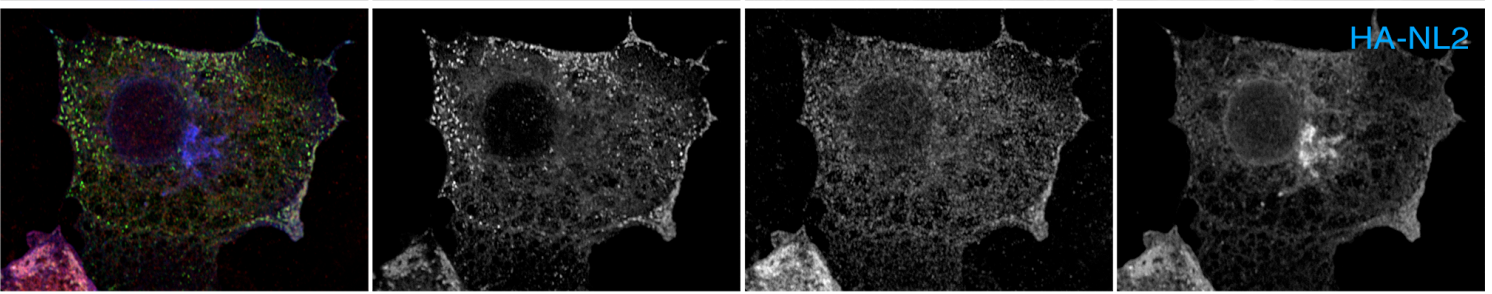

C
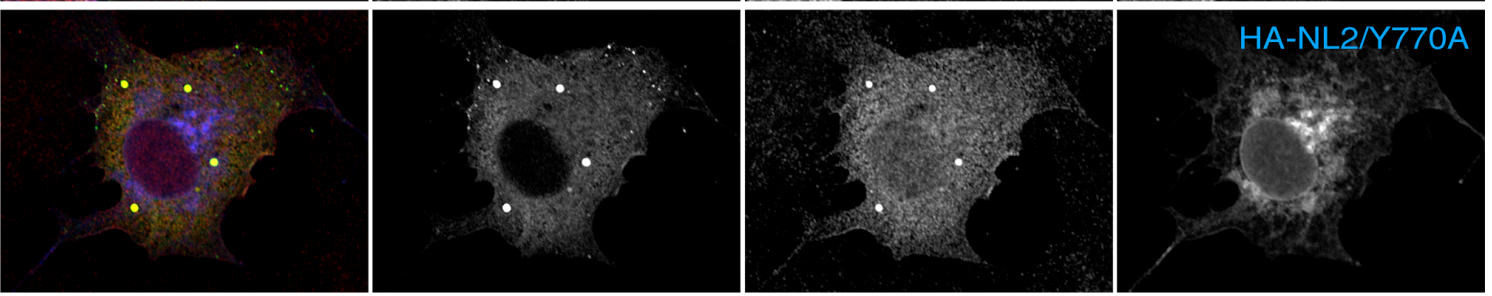

D
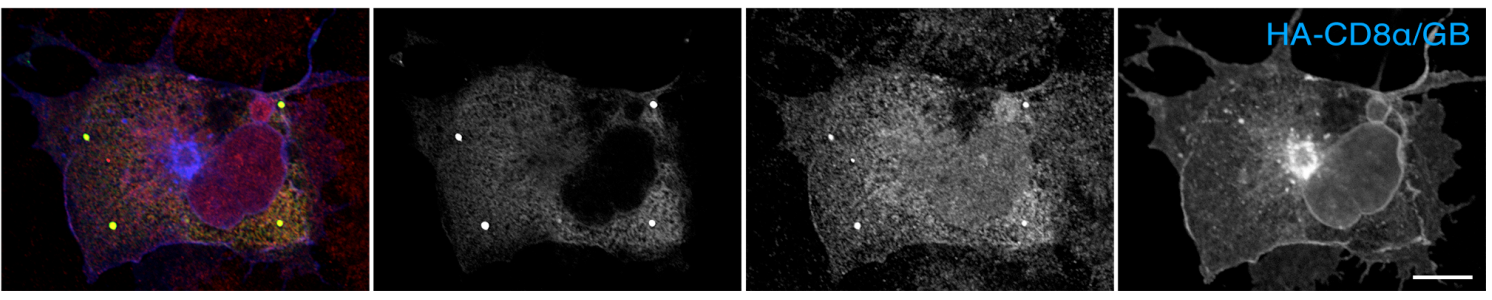

Figure 19: NL2 activates the Collybistin-mediated targeting of Gephyrin to the plasma membrane in heterologous cells. COS7 cells were cotransfected with GFP-Gephyrin (green), myc-CB2SH3+ (red) and either HA-CD8a (A), HA-NL2 (B), HA-NL2/Y770A (C), or HA-CD8a/GB (D) constructs (blue). In the absence of functional NL2, GFP-Gephyrin remains sequestered with myc-CB2SH3+ in cytoplasmic aggregates. In the presence of NL2, GFP-Gephyrin adopts membrane microaggregate distribution and all three proteins cocluster at plasma membrane sites. For this effect, Gephyrin-binding is necessary (C), but not sufficient (D). Scale bar: $20 \mu \mathrm{m}$. 


\subsubsection{Neuroligin 2 activates membrane association of native Collybistin}

In vivo, all Collybistin variants detectable at the protein level (CB1, $\mathrm{CB}_{2 \mathrm{SH}}+$ or $\mathrm{CB}_{\mathrm{SH}_{3+}}$ ) bear a $\mathrm{SH} 3$ domain which inhibits their ability to translocate Gephyrin to submembrane microaggregates (Harvey et al., 2004; Kins et al., 1999; Papadopoulos et al., 2007). Instead, upon cotransfection with Gephyrin in heterologous cells, SH3-containing variants redistribute within large Gephyrin cytoplasmic aggregates (Figure 19 A). This may indicate that a neuronal component could act as an activating cofactor in Collybistin mediated Gephyrin membrane targeting.

As NL2 appears to associate with the Gephyrin-Collybistin membrane complex, the influence of NL2 was examined in respect to the native, SH3-containing splice variant of Collybistin. Strikingly, introduction of HA-NL2 reorganized GFP-Gephyrin and myc-CB2sH3+ into membrane microaggregates (Figure $19 \mathrm{~B}$ ). This indicates that NL2 influences the SH3 domain of Collybistin, disinhibiting its activity towards Gephyrin. In contrast, the Gephyrin-binding-deficient HA-NL2/Y770A was not able to trigger the induction of Gephyrin-CB2 ${ }_{\mathrm{SH} 3+}$ membrane microaggregates (Figure $19 \mathrm{C}$ and $\mathrm{D}$ ). It thus appears that the NL2-Gephyrin interaction is implicated in the activation of Collybistin. As the chimeric CD8a construct bearing the Gephyrinbinding motif $\mathrm{HA}-\mathrm{CD} 8 \alpha / \mathrm{GB}$ was similarly unable to activate Collybistin, NL2specific sequences distinct from the Gephyrin-binding motif seem to be involved in the activation mechanism. This is further corroborated by the finding that myc-NL1 and HA-NL3, despite having the capacity to associate with Gephyrin (Figures 11 and $15 \mathrm{~A}$ ) did not have an activating effect on Gephyrin-Collybistin membrane targeting (Figure 20). Taken together, these data show that NL2 specifically activates Collybistin-mediated Gephyrin membrane targeting, having potentially important implications for NL2 specificity at inhibitory synapses in vivo. The activation mechanism involves, but is not limited to the interaction of NL2 with Gephyrin. 


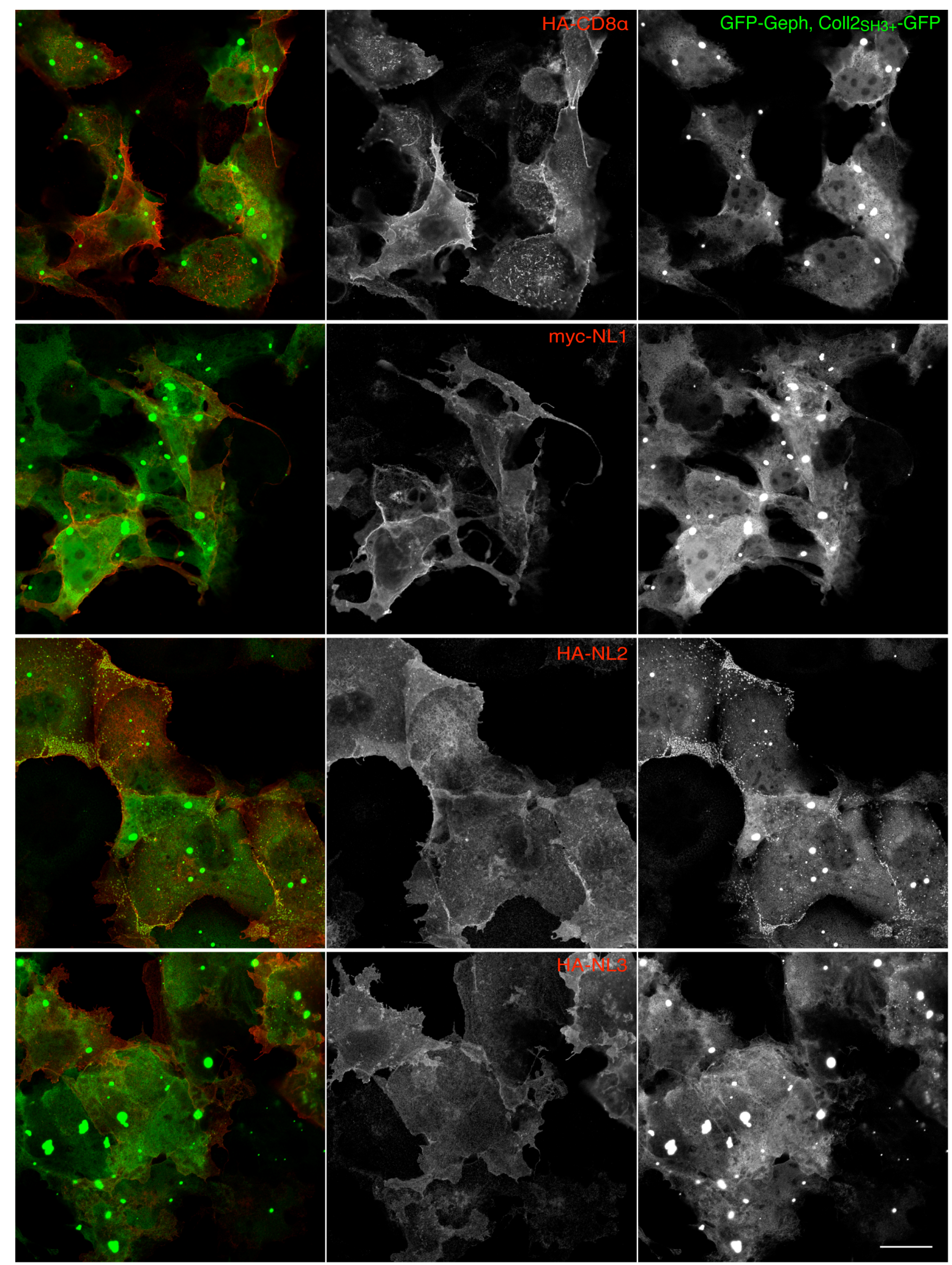

Figure 20: NL1 and NL3 do not activate the Collybistin-mediated targeting of Gephyrin to the plasma membrane in heterologous cells. COS7 cells were cotransfected with GFP-Gephyrin (green), CB2sH3-GFP (green) and either HA-CD8a (top panels), myc-NL1 (middle upper panels), HA-NL2 (middle lower panels) or HA-NL3 (bottom panels) (red). While HA-NL2 induces GFP membrane microaggregates, HA-CD8a, mycNL1 and HA-NL3 do not. Scale bar: $20 \mu \mathrm{m}$. 


\subsubsection{Gephyrin recruitment to somatic postsynaptic sites is disrupted}

\section{in Neuroligin 2-deficient neurons}

The in vitro data presented above point to a mechanistic involvement of NL2 in the recruitment of the Gephyrin-Collybistin complex to plasma membrane sites. To as-
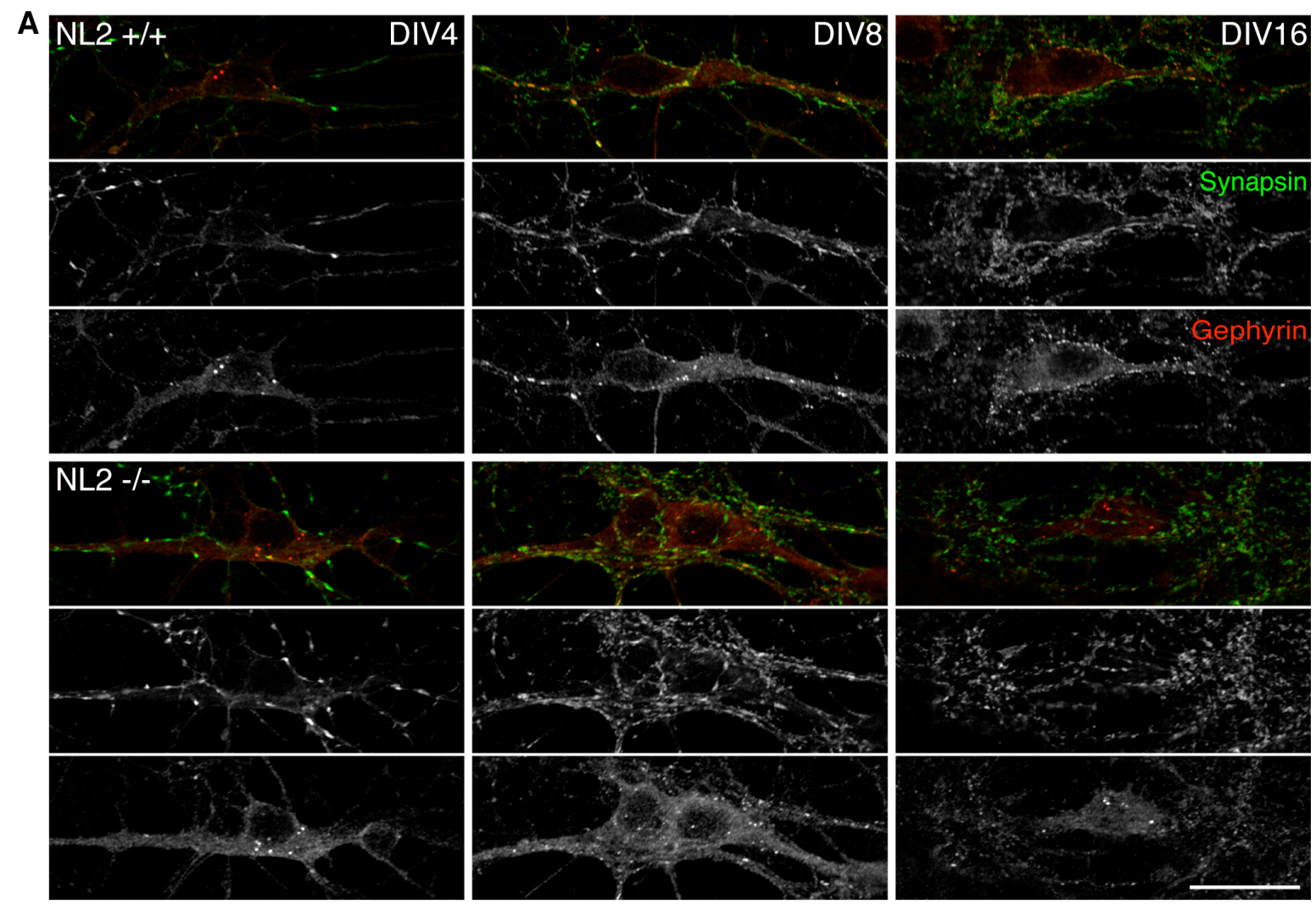

B

C

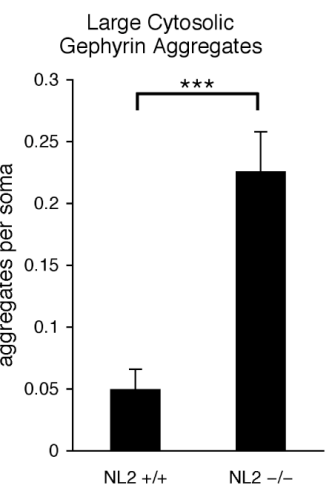

Figure 21: NL2 deficient neurons in culture display aberrant synaptic targeting of Gephyrin. (A) Hippocampal neurons from NL2 +/+ (upper half panels) or NL2 -/- (lower half panels) mice at DIV 4 (left column), DIV 8 (center column) and DIV 16 (right column), stained for endogenous Synapsin 1/2 (green) and Gephyrin (red). Scale bar: $20 \mu \mathrm{m}$. (B) Quantification of the number of postsynaptic Gephyrin clusters on the somatic region per neuron at DIV16. $\mathrm{NL} 2+/+: 8,98 \pm 0,95, \mathrm{~N}=9$ pups, $\mathrm{n}=370$ neurons; $\mathrm{NL2}-/-:$ 4,75 \pm 0,54, $\mathrm{N}=7$ pups, $\mathrm{n}=267$ neurons. Neurons lacking NL2 had significantly less clusters of postsynaptic Gephyrin in the somatic region $(* *, p<0,005$ ). (C) Quantification of the number of cytoplasmic aggregates of Gephyrin per neuron at DIV 16: NL2 +/+: 0,05 $\pm 0,016, \mathrm{~N}=9$ pups, $\mathrm{n}=412$ neurons; NL2 -/-: $0,226 \pm 0,032, N=7, n=307$. Neurons lacking $N L 2$ had significantly more frequent occurrence of cytoplasmic Gephyrin aggregates $(* * *, \mathrm{p}<0,001)$. 
sess the possible involvement of NL2 in the recruitment of the Gephyrin scaffold to postsynaptic sites, hippocampal neurons from NL2 deletion-mutant mice (NL2 -/-) or wild-type (NL2 +/+) littermates were cultured in vitro and fixed at 3 developmental time points (DIV4, 8 and 16). Double staining for endogenous Gephyrin and Synapsin $1 / 2$ as a marker of presynaptic innervation was performed to examine the distribution of Gephyrin in respect to synaptic sites (Figure $21 \mathrm{~A}$ ). In the very early phase of synaptogenesis in wild-type cultured neurons (DIV4), Gephyrin accumulated at postsynaptic sites infrequently, while it was most prominently detected in cytoplasmic aggregates in the soma. Upon development of the culture, synaptic Gephyrin clustering increased while cytoplasmic aggregates were less frequent at DIV8 and DIV16.

Strikingly, in neurons lacking NL2, Gephyrin aggregates persisted until the late stages of the culture, concomitant to a loss of postsynaptic Gephyrin accumulation in the somatic region, despite somata receiving presynaptic innervation (Figure 21 $A$ and B). Although synaptic clustering was severely affected by the loss of NL2 in the somatic compartment, dendritic Gephyrin clusters appeared largely intact. This may imply a difference in the recruitment mechanisms of the somatic versus the dendritic compartment. Overall, this phenotype of NL2-deficient neurons in culture indicates a role for NL2 in the process of Gephyrin recruitment from cytoplasmic deposits to the postsynaptic membrane, at least in the somatic compartment.

\subsubsection{Involvement of Neuroligin 2 in the recruitment of GABAA recep- tors to postsynaptic sites}

Exogenous NL2 clustering by antibody treatment has been shown to co-cluster $\mathrm{GABA}_{A}$ receptors in heterologous cells (Dong et al., 2007). As NL2 is spontaneously clustered on the plasma membrane of cells co-expressing Gephyrin and Collybistin, it seemed plausible that in this cell system $\mathrm{GABA}_{\mathrm{A}}$ receptors may spontaneously co-cluster without the need for exogenous clustering agents.

COS7 cells were co-transfected with six expression plasmids containing HA-NL2,

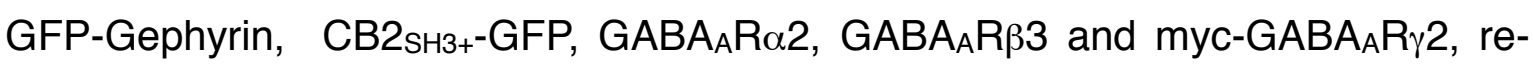
ceptor subunits shown previously to assemble into functional $G A B A_{A} R$ s in heterologous systems (Dong et al., 2007). Transfected Gephyrin and CB2sH3+ were 

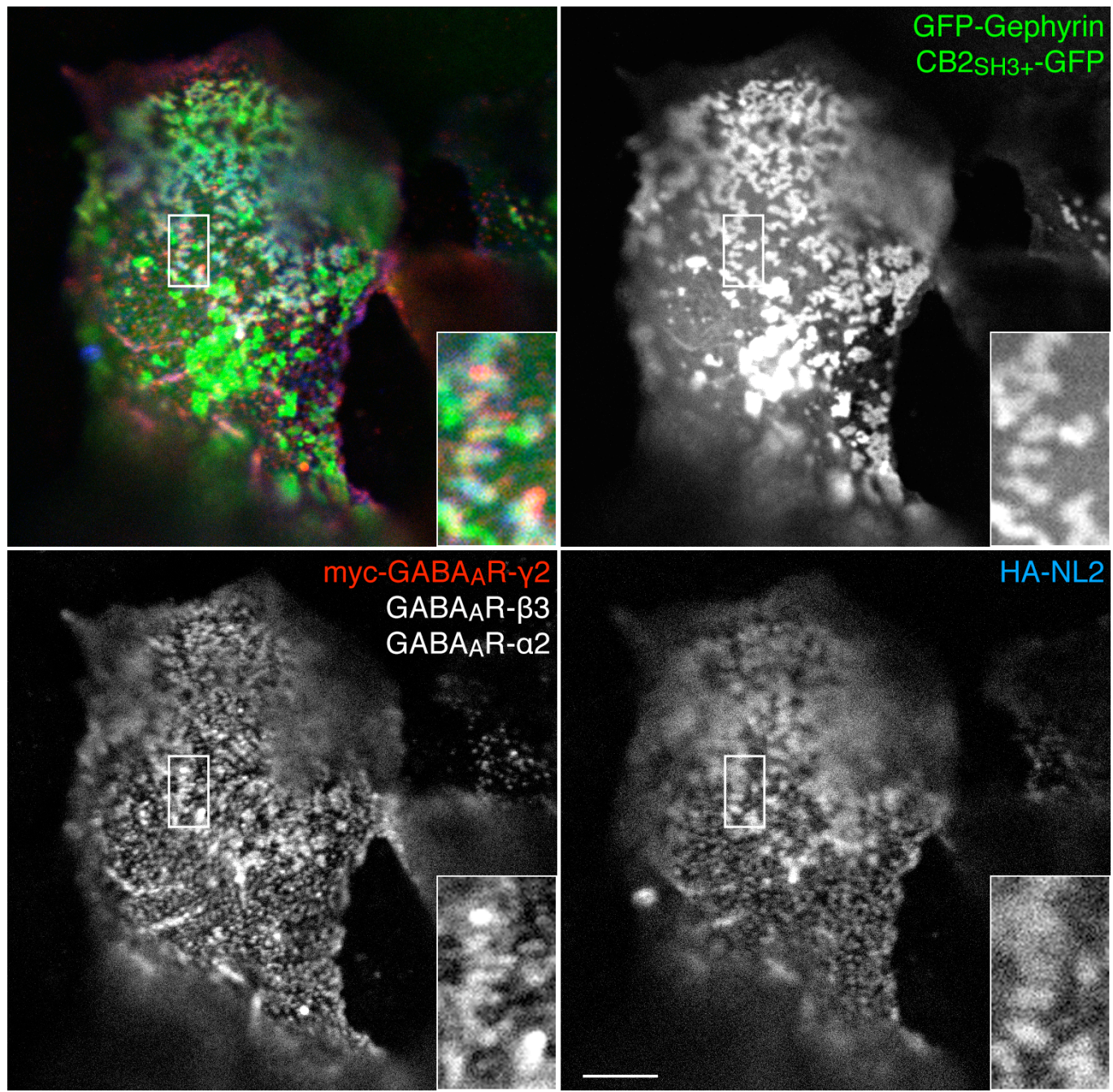

Figure 22: NL2, Gephyrin, Collybistin and GABAA receptors self-organize in heterologous cells. HEK 293FT cells were cotransfected with HA-NL2, GFP-Gephyrin, CB2 ${ }_{\mathrm{SH} 3}-\mathrm{GFP}, \mathrm{GABA}_{\mathrm{A}} \mathrm{R}$ a2 subunit, $G A B A_{A} R B 3$ subunit and myc-GABA $A$ Y2 subunit. Cells were fixed and surface stained for HA (blue) and myc (red). Cytoplasmic GFP fluorescence was also detected (green). Clusters in which all components are enriched are observed at the plasma membrane. Scale bar: $5 \mu \mathrm{m}$, main panel; 1,66 $\mu \mathrm{m}$, inset.

visualized through GFP fluorescence, together with post-fixation surface immunolabeling of $\mathrm{HA}-\mathrm{NL} 2$ and myc-GABA $\mathrm{R} \gamma 2$. Myc surface staining is expected to label complete $\mathrm{GABA}_{\mathrm{A}}$ receptor pentamers as the $\gamma 2$ subunit alone is not expected to traffic to the plasma membrane (reviewed in Barnes, 2000). In cotransfected cells these proteins were observed to spontaneously cocluster and form plasma membrane-associated patches where all three fluorescent signals were coenriched (Figure 22), indicating that NL2-Gephyrin-Collybistin membrane complexes can recruit $\mathrm{GABA}_{\mathrm{A}}$ receptors.

In this heterologous system, partially cotransfected cells cannot be distinguished from six-way transfected cells, rendering a negative readout non-informative. Thus 
A

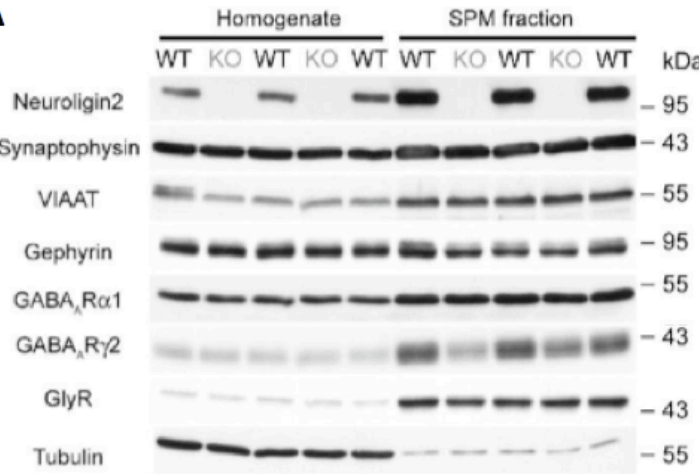

B

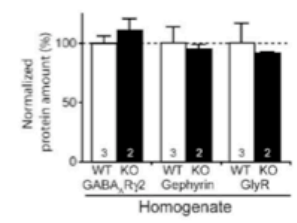

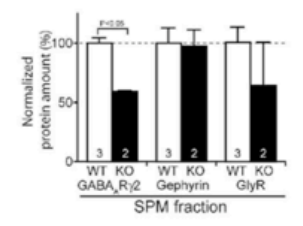

Figure 23: Synaptic protein enrichment in NL2-deficient mice. (A) Brain homogenate (left five lanes) and synaptic plasma membrane (SPM fraction, right five lanes) preparations were immunoblotted for the indicated proteins. Each lane represents pooled sample from three mice whose genotypes are indicated (wild-type=WT, NL2-deletion mutant=KO). Molecular weight markers are indicated in KDa on the left. (B) Infrared band intensity quantification normalized to wildtype levels. Significant differences between wild-type and NL2-deletion mutant samples are indicated above the bar chart.

this system was not suitable for use as an assay to examine which of the exogenous proteins are crucial for receptor clustering. This observation does however serve as a proof of principle that this set of adhesion, scaffolding and receptor proteins is sufficient to cell-autonomously organize into plasma membrane specializations, much like in their native organization at the postsynaptic membrane.

The above in vitro data point to a role of NL2 in the synaptic recruitment of GABA receptors. To study a potential in vivo manifestation of this putative role of NL2, the synaptic content of $\mathrm{GABA}_{\mathrm{A}}$ receptors was examined in NL2 -/- mice. Synaptosomal and synaptic plasma membrane (SPM) samples were prepared from forebrain material of wild-type and NL2 -/- animals. The enrichment of synaptic proteins was examined by SDS-PAGE and immunoblot analysis in the synaptic fractions versus total brain homogenates. Infrared fluorescent quantification of band intensity with the expected electrophoretic mobility showed a significant decrease in the synaptic enrichment of $\mathrm{GABA}_{A}$ receptor subunit $\mathrm{Y} 2$ in the NL2-/- samples versus the wildtype controls (Figure 23). Similar quantification for $\mathrm{GABA}_{\mathrm{A}}$ receptor subunits a1 and Gephyrin only showed a non-significant tendency of being de-enriched in synaptic preparations of NL2-/- animals. However these proteins were not highly enriched in the presented synaptic preparations, making readouts difficult to relate to their synaptic recruitment. Glycine receptor (GlyR) enrichment showed no alterations between the two genotypes, an observation that is difficult to reconcile with perturbations in Gephyrin function as GlyRs are directly influenced by Gephyrin (Meyer et al., 1996; Kneussel et al., 1999). This discrepancy with previous studies 
may be due to the fact that the data set presented concerns preparations of forebrain, a region where glycinergic transmission is not prominent or canonical (reviewed by Lynch, 2004). As $\mathrm{GABA}_{\mathrm{A}}$ receptor subunit $\mathrm{\gamma} 2$ is the subunit most specifically associated with synaptic receptors mediating phasic inhibition versus nonsynaptic receptors mediating tonic inhibition (reviewed by Lüscher and Keller, 2004), this observation can be taken to signify a general reduction of synaptic $\mathrm{GABA}_{\mathrm{A}}$ receptors rather than a specific switch in subunit composition. Taken together with the data from heterologous cells, these observations indicate that NL2 functions to recruit $G A B A_{A}$ receptors to postsynaptic sites.

\subsection{Study on the nature and assembly of Neuroligin oligomers}

At inhibitory synapses, NL2 is ubiquitously present (Varoqueaux et al., 2004). Recent evidence additionally indicates that a minor subset of GABAergic synapses may also contain NL1 and NL3 (Levinson et al., 2005; Budreck et al., 2007). Thus Neuroligin paralogues other than NL2 may additionally function at some inhibitory synapses. The sequence homology of the Neuroligin extracellular domain to Acetylcholinesterase indicates that Neuroligins may form oligomers via in cis interactions at the plane of the membrane. Indeed, in vitro studies with recombinantly expressed soluble Neuroligin extracellular domains indicate that these molecules oligomerize in solution forming homotetramers (Dean et al., 2003) or homodimers (Comoletti et al., 2003; Comoletti et al., 2006; Comoletti et al., 2007 Araç et al., 2007; Chen et al., 2007; Fabrichni et al., 2007; Koehnke et al., 2008). One question that remains to be addressed is the possibility of heterooligomer formation, a mechanism that may implicate various Neuroligin paralogues at inhibitory synapses.

To obtain insight into the physiological relevance of Neuroligin oligomerization observed in vitro, the oligomerization state of native Neuroligins was examined on the surface of neurons in culture using chemical crosslinking. The isolation of Neuroligin oligomers allowed for the characterization of their stoichiometry and composition. Additionally, the implications of oligomerization were examined in terms of 
protein trafficking to determine whether the postsynaptic cell pre-assembles Neuroligin oligomers, or whether oligomers form at the synapse with the participation of the presynaptic component.

\subsubsection{Neuroligins appear as dimers on the neuronal surface}

The isolation and detection of full-length Neuroligin oligomers from cellular preparations is hampered by the need to extract membrane proteins without disturbing putative in cis complexes. At the same time, it is necessary to preserve complex stoichiometry in order to discriminate tightly linked oligomers from multiple Neuroligin molecules within the same detergent micelle. To examine potential Neuroligin oligomers in the context of the neuron, there are additional obstacles pertaining to the fact that Neuroligins at the synapse are very well connected to other synaptic protein complexes. Isolation of Neuroligin oligomers would entail the selective preservation of the in cis homotypic interactions while disrupting the interaction of the Neuroligin CDs with the PSD and of the Neuroligin extracellular domains with presynaptic Neurexins.

To address the above points, a biochemical methodology based on covalent crosslinking was applied on cultured rat hippocampal neurons. Neurons were treated with a solution containing EDTA, in order to selectively interfere with the $\mathrm{Ca}^{2+}$-dependent interactions of Neuroligins with Neurexins, and the homobifunctional crosslinker $\mathrm{BS}^{3}$. $\mathrm{BS}^{3}$ is polar and does not cross the plasma membrane of intact cells. It would thus be expected to covalently link proteins that interact on the neuronal surface or, in the context of the synapse, the synaptic cleft. Subsequently, neurons were lysed with SDS to extract the PSD. As intracellular protein complexes are not expected to be crosslinked, SDS would release Neuroligins from the postsynaptic scaffold. Lysates were analyzed by SDS-PAGE on a 3-8\% gradient tris-acetate gel and blotted for endogenous NL1 (Figure 24 A). NL1 immunoreactivity after crosslinking shifts from the NL1 monomer band, as it appears in lysates of untreated neurons, to a crosslinked adduct with an electrophoretic mobility corresponding to approximately $230 \mathrm{kDa}$. As no other bands were prominent, this indicates that NL1 is extensively complexed extracellularly with a protein of approximately $120 \mathrm{kDa}$, consistent with the notion of NL1 in cis dimerization. 


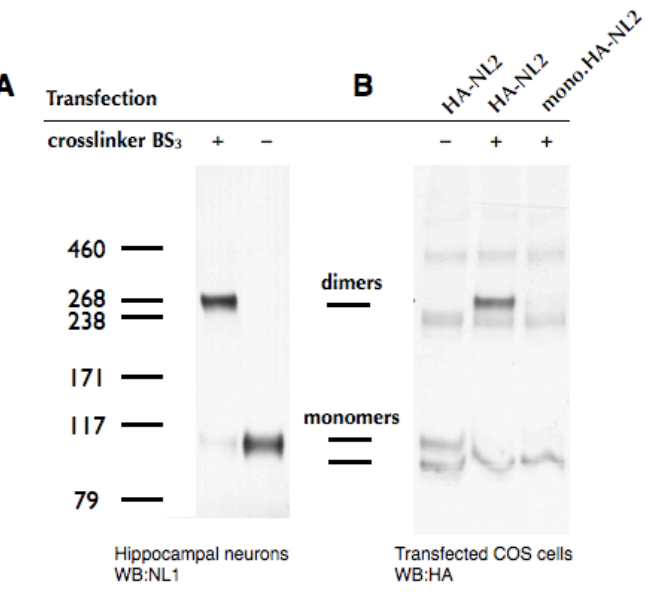

Figure 24: Neuroligin oligomer stoichiometry. (A) Immunoblot detection of endogenous $\mathrm{NL} 1$ in hippocampal neurons treated in situ with crosslinker (lanes +) or left untreated (lanes -). (B) Detection of HA-tagged proteins of COS7 transfected with HA-NL2 (lanes 1-2) or its monomeric mutant E603A/L604A (mono.HA-NL2) and treated in situ with crosslinker (lanes 2-3) or left untreated (lane 1). Molecular weight markers are indicated on the left in KDa. Anticipated Neuroligin monomer and dimer bands are indicated.

To examine whether the crosslinking protocol employed detects Neuroligin oligomers with the properties that have previously been attributed to Neuroligin oligomers in solution, a double point mutation in the extracellular AChE domain (E603A/L604A), homologous to one reported to interfere with NL1 oligomerization in solution (Dean et al., 2003), was introduced into HA-NL2 (HA-monoNL2). COS7 cells were transfected with HA-NL2 or HA-monoNL2 and similarly treated with BS 3 . Crosslinked HA-NL2 formed adducts migrating at approximately $240 \mathrm{kDa}$, while HA-monoNL2 was not detected in higher order adducts (Figure $24 \mathrm{~B}$ ). As this mutation, predicted to interfere with oligomerization, abolishes the major Neuroligin adduct, it indicates that in this heterologous system, NL2 homodimerizes and the homodimers are detected as a crosslinked adduct of approximately $240 \mathrm{kDa}$. This further supports the notion that the NL1-containing adduct of approximately $230 \mathrm{kDa}$ detected from crosslinked neuron preparations represents Neuroligin dimers from the neuronal surface.

\subsubsection{Neuroligin dimers can be heteromeric and have specific para- logue combinations}

Neuroligin dimers observed in solution have been homomeric as in each of these in vitro studies a single recombinant Neuroligin paralogue was included. However, due to the extensive homology between Neuroligin paralogues within the extracellular AChE domain reported to mediate oligomerization, the possibility remains that Neuroligins form heterooligomers in neurons where different paralogues are coexpressed. 


\section{A}

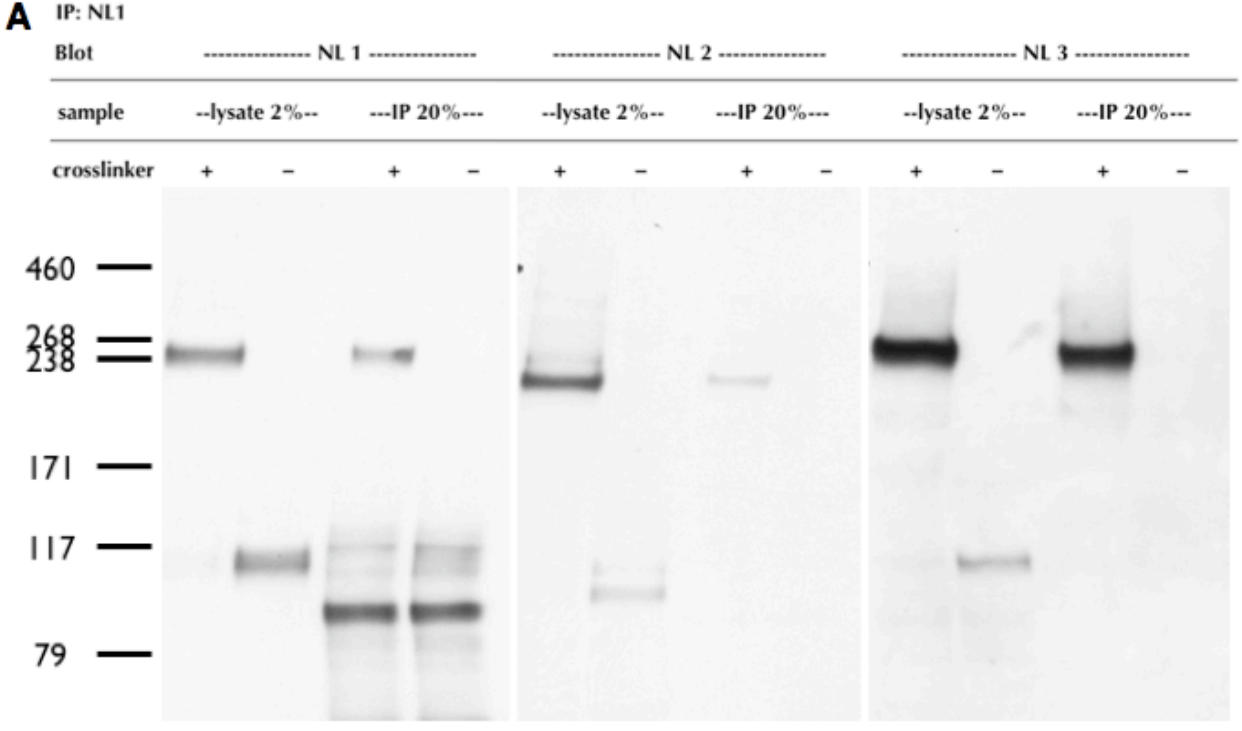

B IP: NL2

\begin{tabular}{|c|c|c|c|c|c|c|}
\hline Blot & N & ......... & 1 & 2 ……… & 1 & - ..-- \\
\hline sample & -lysate $2 \%--$ & -.-IP 20\% & -lysate $2 \%$ & -.-IP $20 \%$ & -lysate $2 \%$ - & $\ldots$ IP $20 \% \ldots$ \\
\hline crosslinker & + & + & - & + & - & + \\
\hline
\end{tabular}

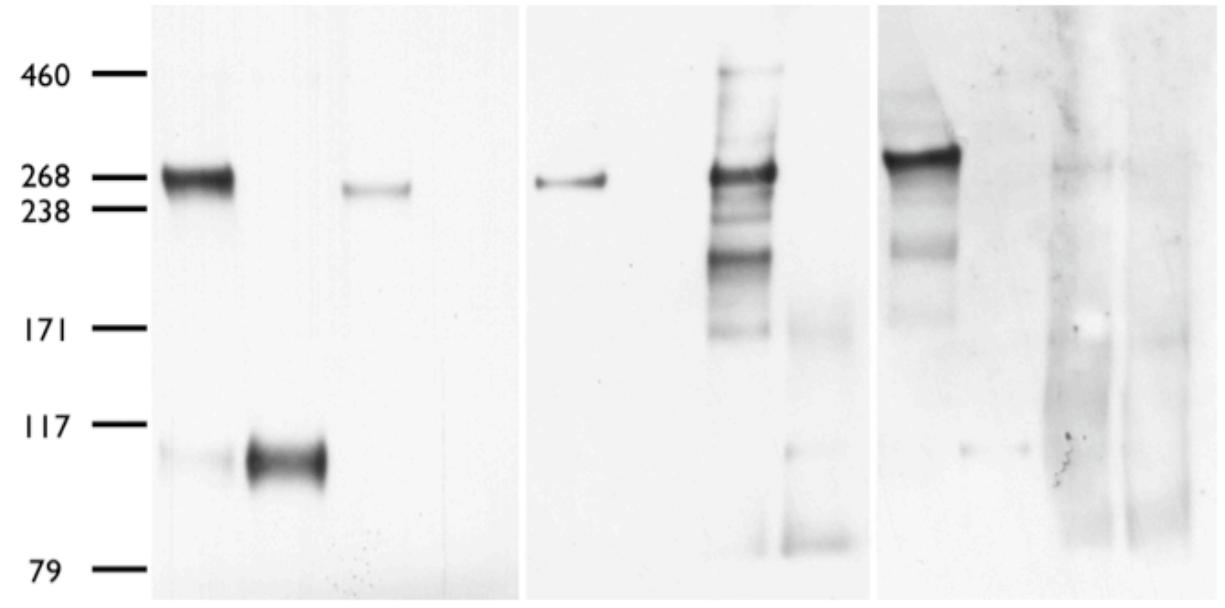

C IP: NL3

\begin{tabular}{llllllllll} 
Blot & & & & & & & & & \\
\hline
\end{tabular}

${ }^{400}-$

${ }_{238}^{268}=$

1171-

$112-$

79
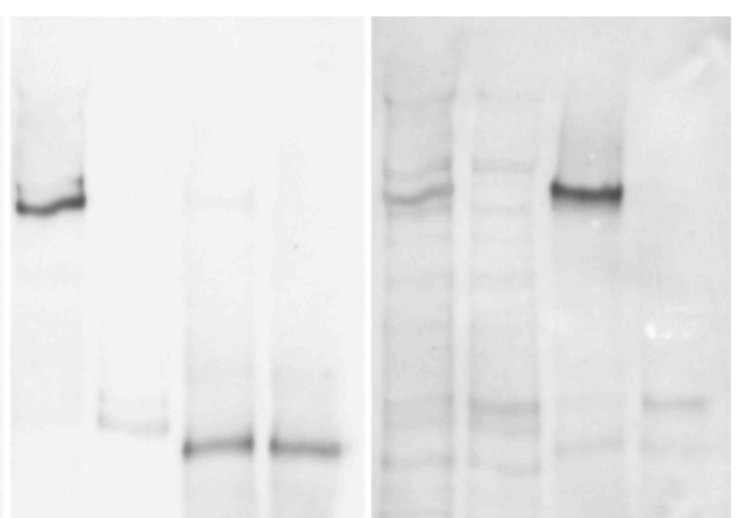

Figure 25: Neuroligin dimer composition: (A) Immunoblots for endogenous NL1 (left panel), NL2 (center panel) and NL3 (right panel) of lysates (left two lanes of each panel) or immunoprecipitates (right two lanes of each panel) of endogenous NL1 (A), NL2 (B) or NL 3 (C) from hippocampal neurons in culture treated with crosslinker $(+)$ or left untreated (-). Molecular weight markers are indicated in $\mathrm{kDa}$. 
To determine the paralogue composition of Neuroligin dimers in neurons, Neuroligin crosslinked adducts of a specific paralogue were purified and analyzed for the presence of the other Neuroligin paralogues. Lysate, containing 1\% SDS, from hippocampal neurons treated with $\mathrm{BS}^{3}$, as described above, was diluted with 7 volumes of $1 \%$ TritonX-100 solution. A 1:7 detergent ratio of SDS to TritonX-100 produces a mixed micelle lysate which does not denature antibodies or interfere with immunoprecipitation procedures (Leonard et al., 1998). Immunoprecipitation procedures were carried out on these preparations individually with paraloguespecific antibodies for NL1, 2 and 3. NL4 was not detected in the lysates.

Immunopurified NL3-containing crosslinked adducts were analyzed by gradient SDS-PAGE and immunoblotted for the 3 aforementioned endogenous Neuroligin paralogues (Figure $25 \mathrm{C}$ ). Apart from the anticipated NL3 immunoreactivity in the bands putatively corresponding to Neuroligin dimers, robust NL1 immunoreactivity was also observed in the putative dimer band in these samples. In equivalent NL3immunoprecipitates that were not crosslinked, NL1 was not detected, demonstrating that potential cross-reactivity of Neuroligin paralogue-specific antibodies does not lead to detectible immunoreactivity and could not account for the double immunoreactivity of the dimer band. The converse procedure yielded compatible results as immunoprecipitation of NL1-containing adducts exhibited robust NL3 immunoreactivity in immunoblots (Figure $25 \mathrm{~A}$ ). As this surface crosslinked adduct of approximately $230 \mathrm{kDa}$ is immunoreactive for both NL3 and NL1, it may be concluded that this band represents a discrete NL1/3 crosslinked heterodimer.

In the NL3 immunopurified crosslinked adducts, NL2 immunoreactivity was not observed (Figure $25 \mathrm{C}$ ) and, equivalently, NL3 was not detected in NL2immunopurified adducts (Figure $25 \mathrm{~B}$ ). This indicates that in hippocampal neurons in culture, NL2/3 heterodimers are not prominent. NL1 immunoreactivity was faintly detected in NL2-containing dimers, as was NL2 in NL1-containing dimers (Figures $25 \mathrm{~B}$ and A). This observation speaks of potential NL1/2 heterodimers which are far less abundant than other species. As NL2-containing adducts do not show prominent immunoreactivity for any of the other Neuroligin paralogues, it may be concluded that in hippocampal neurons in culture, surface NL2 predominantly forms homodimers. 


\subsubsection{Neuroligin monomers are retained in the early secretory path- way via a transmembrane retention signal}

To study the physiological significance of Neuroligin dimerization in neurons, the dimerization-deficient mutant construct HA-monoNL2 was used to transfect hippocampal neurons in culture. $24 \mathrm{~h}$ after transfection, HA immunoreactivity was only detected in the somatic remnants of dead neurons, while non-expressing neurons in the rest of the culture appeared healthy. This was in striking contrast to the effect of transfection of HA-NL2 where neurons robustly expressed the protein on the plasma membrane, indicating that the E603A/L604A double point mutation was enough to hinder proper expression and/or confer toxicity to the protein product.

A heterologous expression system was used instead of neurons to examine the expression properties of monomeric Neuroligin constructs. HA-monoNL2 was used to transfect COS7 cells. After $12 \mathrm{~h}$ of expression, transfected cell lysates were analyzed by SDS-PAGE and HA immunoblotting. The band pattern observed for the monomeric mutant differed from that of unmutated HA-NL2; the characteristic band pattern of tagged Neuroligins in cell-linescell-lines exhibited an altered intensity ratio where HA-monoNL2 showed decreased levels of the slower migrat-

A

\begin{tabular}{|c|c|c|c|c|c|c|c|c|c|}
\hline Transfection & \multicolumn{3}{|c|}{ HA-NL2 } & \multicolumn{3}{|c|}{ mono. $H A-N L 2$} & \multicolumn{3}{|c|}{ mono.HA-NL2-N701L } \\
\hline Endo $\mathrm{H}$ & - & + & - & - & + & - & - & + & - \\
\hline PNGase $F$ & - & - & + & - & - & + & - & - & + \\
\hline & $=$ & $=$ & & son & $x=$ & & & & \\
\hline
\end{tabular}

B

NL2 LSVTVAVGASLLFLNILAFAALY

Pen2 YYLGGFAFLPFLWLVNIFWFF

C

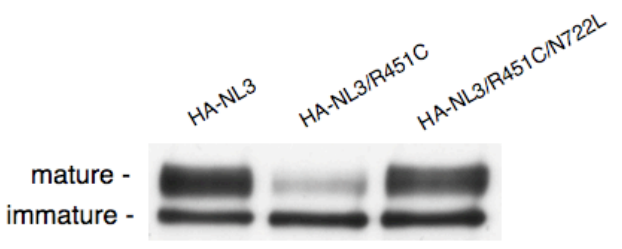

Figure 26: Neuroligin monomer retention. (A) Immunoblot detection ofr HA from lysates of COS7 cells transfected with either HA-NL2 (left), its monomeric mutant E603A/L604A (mono.HA-NL2; center) or a combined monomeric/transmembrane mutant E603A/ L604A/N701L (mono.HA-NL2-N701L; right). Endoglycosidase $\mathrm{H}$ (Endo $\mathrm{H}$ ) and PNGase $\mathrm{F}$ treatments are indicated $(+)$. (B) Comparison of the transmembrane domain of NL2 with the first transmembrane domain of Pen2. Asparagine residues that affect trafficking are in red. (C) Immunoblot detection for HA from lysates of COS7 cells transfected with either HA-NL3 (left), its autism-related mutant R451C (center) and a combined autismrelated and transmembrane mutant of R451C/ N722L (right). 
ing diffuse HA-immunoreactive band compared to the faster migrating and more compact immunoreactive band duplet (Figure $24 \mathrm{~B}$, lanes 1 and 4).

As is often the case with membrane glycoproteins, Neuroligins exhibit electrophoretic segregation of their different glycosylation species (Comoletti et al., 2003). To determine whether the band pattern may represent the state of glycosylation, transfected cell lysates were treated with endoglycosidases. Treatment of lysates with Endoglycosidase $\mathrm{H}$ caused the shift of the fast-migrating compact band duplet to a single compact band of higher electrophoretic mobility, leaving the heavier diffuse band unchanged (Figure $26 \mathrm{~A}$, lanes 2 and 5). As Endoglycosidase $\mathrm{H}$ cleaves off immature $\mathrm{N}$-linked glycans added in the endoplasmatic reticulum (ER) early in the secretory pathway, this indicated that the lower band duplet in the lysate represents ER resident species of the tagged proteins.

Treatment of the lysate with PNGase F resulted in an equivalent collapse of the duplet band as with Endoglycosidase $\mathrm{H}$, while it additionally increased the mobility of the heavier diffuse band converting it to a new, more compact band with a mobility between the fastest and slowest migrating bands observed in this experiment (Figure 26 A, lanes 3 and 6). PNGase F cleaves both immature and mature Nlinked glycans alike, stripping glycans originating from both ER and trans-Golgi processing. The heavier diffuse band, being resistant to Endoglycosidase $\mathrm{H}$ treatment but susceptible to PNGase F treatment represents maturely glycosylated protein that has reached the late compartments of the secretory pathway. The fact that the heavier diffuse band did not collapse onto the fastest migrating band, presumably representing the unglycosylated species, is in line with the observation that Neuroligins are also modified with O-linked glycans (Ichtchenko et al., 1995) in the later secretory compartments which are resistant to cleavage by either of the endoglycosidases employed.

Experiments with endoglycosidase treatment indicate that the band profile of HANL2 expressed in COS7 cells is indicative of their glycosylation state which correlates with the progression of trafficking through the secretory pathway. That HAmonoNL2 exhibits decreased amounts of maturely glycosylated protein versus the amount of ER-resident species can be taken as indication of a trafficking deficit of the monomeric form of NL2 through the secretory pathway. This deficit could arise 
from protein misfolding of the mutant Neuroligin variant that would lead to faulty post-translational processing and degradation. Alternatively, a receptor for Neuroligin monomers could be involved in identifying and retaining monomers in the early compartments of the secretory pathway.

The notion of assembly control of in cis membrane protein complexes is not novel and is prominent in synaptic proteins such as ionotropic receptors and voltagegated ion channels (reviewed by Ma \& Jan, 2002). In these cases, consensus motifs in the primary protein sequence serve as ER-retention signals that actively limit the trafficking of subunit monomers through the secretory pathway. Increasing expression of HA-monoNL2 by allowing for longer expression times resulted in the enrichment of the maturely glycosylated species of monomer over the immature species (data not shown). This observation is indicative of the involvement of a saturatable process in the trafficking deficiency of monomers, supporting the notion of the existence of an active retention mechanism that would have a capacity limit for monomer retention rather than a passive process where the monomeric mutations lead to protein misfolding and subsequent deficient secretory processing.

Following the idea of an active retention mechanism for Neuroligin monomers, the primary sequence of NL2 was examined for regions of consensus with identified retention motifs. While classical such consensus sequences were not identified, NL2 does posses a conserved asparagine residue in the midst of its hydrophobic transmembrane stretch. This feature of the transmembrane domain, common to the other Neuroligin paralogues, is reminiscent of the structure of the first transmembrane domain of Pen2 (Figure $26 \mathrm{~B}$ ), a subunit of the $\mathrm{Y}$-secretase membrane complex. Pen2 contains an asparagine residue within the first transmembrane domain which is involved in its retention in early secretory pathway compartments as a monomer. In cis interactions of Pen2 leading to membrane complex assembly results in the release of Pen2 to traffic through the secretory pathway towards the plasma membrane (Kaether et al., 2007).

To examine the possibility that the conserved transmembrane asparagine is involved in the ER retention of monomeric NLs, this residue was mutated to a hydrophobic residue and the effects on trafficking were examined. Mutation of N701 
to leucine was introduced to the monomeric NL2 variant resulting in the HAmonoNL2/N701L mutant. The glycosylation profile of this variant was examined in parallel with the wild-type and monomeric variants of HA-NL2 in COS7 cells (Figure $26 \mathrm{~A}$, lanes 7 to 9). While HA-monoNL2/N701L carries the monomeric mutations, its glycosylation profile was indistinguishable from that of the unmutated variant, indicating that mutation of N701 to leucine effectively reverses the ERretention effect of the monomeric mutations. Similar observations were made with the homologous mutants of myc-NL1 (data not shown). Take together, these data implicate the transmembrane asparagine residue in a mechanism of retention in the early secretory pathway of Neuroligin monomers, potentially equivalent to the mechanism described by Kaether et al. (2007) for the ER-retention of Pen2 prior to membrane complex assembly.

A NL3 mutation spontaneously occurring in humans associated with autism spectrum conditions (Jamain et al., 2003) and causing autism-related behavior in genetically modified mice (Tabuchi et al., 2007) has been predicted to interfere with Neuroligin oligomerization (De Jaco et al., 2006) and shown to induce ER retention (Chih et al., 2004; Comoletti et al., 2004; Chubykin et al., 2005; De Jaco et al., 2006). As the above observations imply a link between the inability to dimerize and ER-retention, autism-related NL3 mutant R451C was examined for the involvement of the transmembrane asparagine-dependent retention mechanism. Expressed in COS7 cells, HA-NL3/R451C exhibited a reduced ratio of mature versus immature protein compared to HA-NL3, in line with previous observations of ERretention of this mutant. Mutation of the transmembrane asparagine of the autismrelated mutant reversed the effects on trafficking as HA-NL3/R451C/N722L exhibited a ratio of mature versus immature protein similar to that of the unmutated variant (Figure $26 \mathrm{C}$ ). This indicates that the transmembrane asparaginedependent mechanism of monomer retention in the ER identified in this study is involved in the faulty protein processing reported for the autism-associated mutation of NL3. 


\section{Discussion}

The questions that provoked the study presented herein revolve around the phenomena implicated in the deployment of the postsynaptic machinery during the formation of inhibitory synapses. There has recently been an accumulation of biochemical, cell biological and genetic evidence that, despite some inconsistencies in the interpretations, strongly converges to indicate a central role for the Neuroligin family of postsynaptic adhesion molecules in the development of the postsynaptic apparatus (Ichtchenko et al., 1995; Irie et al., 1997; Dean et al., 2003; Chih et al., 2005; Varoqueaux et al., 2006; Chubykin et al., 2007; Aramuni et al., submitted for publication; reviewed in Craig \& Kang, 2007). The identification of Neuroligin 2 (NL2) as a paralogue specifically located at, and involved in the development of the inhibitory synapse (Varoqueaux et al., 2004; Graf et al., 2004) served as a starting point for the investigation into the mechanisms of inhibitory postsynapse assembly.

The experimental evidence presented herein points to several mechanistic properties of NL2 that allow it to act as a central organizer of the inhibitory postsynapse. During the course of the study, however, it became apparent that NL2 may not have exclusivity among Neuroligins in inhibitory synapse development. The need thus arose to understand the organization of the synaptic armament of Neuroligins in terms of the trafficking and presentation on the postsynaptic membrane. The examination of different Neuroligin paralogues is of additional interest due to the involvement of NL3 and NL4 in monogenic heritable forms of Autism (Jamain et al., 2003; Zoghbi, 2003; Laumonnier et al., 2004; Yan et al., 2004; Tabuchi et al., 2007; Jamain et al., 2008). The experimental evidence provided herein necessitated the expansion of this study to include general properties of Neuroligin function and their potential in the development of the postsynapse.

\subsection{Functional unit of Neuroligins: the dimer}

The development of antibodies that are specific to a particular Neuroligin paralogue has allowed the differential analysis of Neuroligins in terms of the synaptic types with which they associate. Two landmark studies showing that NL1 associ- 
ates with postsynaptic glutamatergic, but not GABAergic synaptic markers (Song et al., 1999), and that NL2 does not associate with glutamatergic markers, but rather consistently localizes to symmetric synapses and colocalizes with inhibitory markers (Varoqueaux et al., 2004), have dictated how Neuroligins are integrated in currently prevailing models of the synapse (reviewed by Dean \& Dresbach, 2005).

The segregation of the paralogues to distinct synaptic types presumes that they are discrete units that traffic to different synapses independently. However, the homology of the Neuroligin extracellular domain with Acetylcholinesterase (AChE) (Dean et al., 2003) indicates that Neuroligins may have the capacity to oligomerize in cis via their AChE domain. Indeed, in vitro experiments with soluble forms of recombinant Neuroligin extracellular domains support this notion, showing that Neuroligin extracellular domains form dimers or tetramers after crosslinking (Dean et al., 2003), spontaneous dimers in solution (Comoletti et al., 2003; Comoletti et al., 2006; Comoletti et al., 2007) and dimeric units in crystals (Araç et al., 2007; Fabrichny et al., 2007; Koehnke et al., 2008).

Data substantiating the notion of Neuroligin oligomerization raise the question of whether Neuroligin paralogues only form homomers, as shown in the above studies, or whether they may appear as heteromers on the postsynaptic membrane. This outstanding question is critical to understand the organization of Neuroligins at the synapse, especially in light of the different synaptic specificities of Neuroligin paralogues. However, studies with recombinant proteins do not have the capacity to address this question, as the composition of endogenous oligomers is not examined.

In the present study, data are presented on the oligomerization state of endogenous Neuroligins in hippocampal neurons in culture. Neurons were treated with calcium chelator to disengage Neuroligins from their presynaptic binding partners, Neurexins, and with a membrane impermeable homobifunctional crosslinker to covalently link surface proteins in situ. Treatment caused a drastic shift in the electrophoretic mobility of Neuroligins 1, 2 and 3 (Figures 24 and 25) consistent with efficient covalent linkage into dimers. Along with the previously mentioned literature on in vitro dimerization of recombinant Neuroligins, this study substantiates the notion that Neuroligins indeed form dimers on the neuronal surface. As the 
crosslinker employed selectively links via lysine residues, the possibility of a structural bias for dimers versus higher order oligomers that may be present on the neuronal surface cannot be excluded by the present dataset. However, the remarkable efficiency of crosslinking, manifested by the diminishing of the monomeric band in treated samples, and the lack of significant bands representing higher order crosslinked adducts indicate that the vast majority of Neuroligins are in a dimeric state and not present as monomers or higher order oligomers.

The detection of Neuroligin-containing complexes with the size of an anticipated dimer does not ascertain that these complexes are indeed composed of two Neuroligin molecules. It is possible that a single Neuroligin molecule was in complex with another synaptic protein of similar size. To disambiguate the interpretation of the above results, the same in situ crosslinking approach was employed in heterologously expressed Neuroligins in non-neuronal cell-lines. A double point mutation close to the predicted dimerization interface (referred to herein with the designation "mono") that has been shown to disturb in vitro oligomerization of Neuroligins (Dean et al., 2003) was included in the study. Crosslinking of heterologously expressed Neuroligins consistently exhibited the same strict pattern of migration corresponding to a dimeric adduct as was observed in neurons, an observation that argues against the participation of an unidentified synaptic protein component in the adducts. Additionally the monoNL2 mutant when similarly treated did not exhibit dimeric or any other crosslinked adduct formation (Figure $24 \mathrm{~B}$ ). As this mutation was shown to interfere with oligomer formation in a purified system, it supports the notion that the appearence of crosslinked adducts stems from the properties of Neuroligins to oligomerize in cis.

The observation that monoNL2 does not exhibit crosslinked adduct formation can indicate either that monoNL2 remains in a monomeric state or was not exposed to crosslinker on the cell surface. The latter possibility was not directly addressed and it is shown herein that monoNL2 does exhibit trafficking deficiencies that lead to lower surface expression. However, monomeric mutants were not observed to be completely absent from the plasma membrane in this heterologous system. Additionally, Dean et al. (2003) report surface expression of a monoNL1 mutant in a similar cell-line system showing it to be biotinylated, thus exemplifying that these mutants are exposed to membrane-impermeable reagents. Consequently, the lack 
of crosslinked adduct formation by monoNL2 is taken as evidence that this mutant remains in a monomeric state. Additionally, this is an indication that the oligomers observed in this study arise from the same phenomena as previously studied in vitro, showing that the results may be directly correlated. The findings herein thus converge with and expand on previous observations with data coming from endogenous full length proteins in neurons. These observations directly address the question of stoichiometry of Neuroligin oligomers on the neuronal surface pointing to a strict in cis dimeric membrane complex.

As in situ crosslinking led to the formation of stable covalently linked dimeric adducts, this approach appeared suitable to address the question of whether Neuroligin dimers can be heteromeric. The composition of endogenous Neuroligin dimers was examined in the neuronal preparation in a combined methodology of immunopurification and immunoblotting with distinct paralogue-specific antibodies. This approach allowed for the examination of the same dimer population for two distinct Neuroligin immunoreactivites. Dimeric adducts immunoprecipitated using NL1-specific antibodies appear to additionally exhibit robust immunoreactivity for NL3 in immunoblot analysis (Figure 25A). Conversely, immunoprecipitates with NL3-specific antibody exhibit NL1-specific immunoreactivity at the band representing dimeric adducts (Figure $25 \mathrm{C}$ ). The electrophoretic mobility of these adducts shows that they represent a maximum of two Neuroligin molecules. Thus, these adducts can be regarded as being characterized in their entirety as NL1/3 heterodimers, excluding the possibility that they are complexes of Neuroligins with other proteins like presynaptic Neurexins or postsynaptic receptors. The detection of NL1/3 heterodimers exemplifies that Neuroligin dimers in neurons can indeed be heteromeric.

Reaffirming the above results, monomeric bands in uncrosslinked samples did not show crossreactivity between different Neuroligin paralogues. Specifically, material immunoprecipitated using NL3-specific antibody is immunoreactive for NL3 at the monomeric band in immunoblots, but not for NL1 (Figure $25 \mathrm{C}$ ). Equivalent observations were made for the other paralogue-specific preparations, testifying to the paralogue-specificity of the antibodies in both immunoprecipitation and immunoblot, thus excluding the possibility that adducts immunoreactive to two paralogue-specific antibodies appear so due to crossreactivity of the antibodies. 
Analysis of the crosslinked neuronal material for NL2 revealed that NL2-containing crosslinked dimers show no detectable immunoreactivity for NL3 and, conversely, NL3-containing dimers show no detectable immunoreactivity for NL2. This indicates that cultured hippocampal neurons do not form NL2/3 heterodimers. NL2 and NL3 have been shown to be expressed in the same set of neurons in hippocampal cultures, and indeed to colocalize in a subset of inhibitory synapses and co-immunoprecipitate from non-ionic detergent extracts of brain (Budreck \& Scheiffele, 2007). Taken together, these data show that while Neuroligins can form heterodimers, the pairing of paralogues is not promiscuous nor is it solely dictated by which Neuroligins co-express in a given neuron, or even by which Neuroligins colocalize to a given postsynaptic complex.

For the particular case of NL2 and NL3, Budreck and Scheiffele (2007) suggest that co-immunoprecipitation of these two paralogues may indicate that they form NL2/3 oligomers. In light of the data presented herein and keeping in mind that non-ionic detergent extraction is known to leave the postsynaptic density largely intact, including that of inhibitory synapses ( $\mathrm{Li}$ et al., 2007), it appears that NL2 and NL3 may, on occasion, reside on the same postsynapse, but in hippocampal cultures do not form heterodimers. As monomeric Neuroligins were not prominently observed on the surface of these neurons (Figure $24 \mathrm{~A}$ and 25), it thus seems that a given synapse is capable of containing more than one species of Neuroligin dimer.

Examination for NL1/2 heterodimers revealed that NL2-containing dimers show weak but detectable immunoreactivity for NL1 and vice-versa (Figure $25 \mathrm{~A}$ and B). This indicates that hippocampal neurons do form NL1/2 heterodimers. The methodology employed cannot provide quantitative information on the prevalence of a certain dimeric species, as the efficiencies of immunoprecipitation of each of the paralogues was not taken into account. Additionally, immunoprecipitation of dimers was more efficient compared to monomers (Figure 25), presumably due to increased avidity coming from double the epitopes in dimers. However, the contrast in readouts of robust detection of NL1/3 dimers versus the lack of detectable NL2/ 3 dimers shows that this methodology has a wide spectrum in terms of signal intensity readout. The readouts are thus interpretable in a semi-quantitative manner. In this respect, it appears that NL1/2 dimeric species are a rarity in hippocampal 
neurons compared to the much more prevalent NL1/3 species. Indeed, NL1 and NL2 largely segregate from each other into excitatory and inhibitory sites in hippocampal cultures, though, on exception, NL1 does appear to colocalize with inhibitory markers (Levinson et al., 2005). This small portion of NL1 at inhibitory synapses, where NL2 is considered omnipresent is consistent with and may correspond to the minor dimeric species of NL1/2. Despite the apparent scarcity of NL1/ 2 dimers, the fact that they were detected nonetheless indicates that there is no inherent incompatibility for this Neuroligin dimer combination.

In hippocampal neurons in culture NL2 does not seem to be frequently paired with other paralogues. As NL4 was not detectable in these preparations, it remains to be seen whether NL2/4 dimers may represent a considerable fraction of NL2containing dimers. Nonetheless, the most straightforward interpretation of these data is that the vast majority of NL2 is in the homodimeric state.

Neuroligin dimers thus occur with diverse -yet specific- compositions. For hippocampal neurons in culture, it seems safe to say that NL1/2, NL1/3 and NL2/2 species form, while it is likely -though not directly observed- that NL3/3 species exist as NL3 is the most abundant of the paralogues in brain (Varoqueaux et al., 2006). Additionally, some form of NL4-containing dimers are also anticipated to exist in vivo. This exemplifies that Neuroligin complexity, in terms of the different properties of the four paralogues and the corresponding attributes that these properties instill a given synapse with, is far greater than envisaged in the current models that deal with Neuroligins as monomers or, equivalently, homodimers. Instead, it appears that the Neuroligin unit is the dimer which can exist in a variety of distinct species -in mice maximally ten.

As seen in hippocampal neurons in culture, the ensemble of dimer species that a given set of neurons is equipped with is defined, and does not encompass every possible dimer combination. As noted previously, this selectivity for specific dimer species does not arise from the selective expression of Neuroligin paralogues as exemplified by the lack of NL2/3 dimers though both are expressed in the same cells. It can thus be argued that there are certain criteria that dictate which dimer combinations may form. These criteria can be either fixed, inherently predetermined by the primary properties of the paralogues, or regulated. In the case of 
NL1/2 dimers, the latter case appears more plausible. The fact that NL1/2 dimers were detected but in proportionately low levels implies that, while the primary properties of the two paralogues do not prohibit their pairing, unidentified regulatory mechanisms favor the formation of other combinations, such as NL1/3. Thus the process of dimer formation may be regulated by the cell, favoring specific Neuroligin combinations over others.

It is critical to understand the rules that regulate Neuroligin dimer composition. Plausible mechanisms that have been documented for the Neuroligin extracellular domain could include splicing events (Ichtchenko et al., 1995, Ichtchenko et al., 1996) or post-translational modifications such as glycosylation (Ichtchenko et al., 1995). Neuroligins 1, 2 and 3 all contain a splice cassette designated "A" which has distinct sequences for NL1 and NL2, while NL3 may contain sequences homologous to either (Ichtchenko et al., 1996). A combination of splicing and posttranslational modification is an especially intriguing possibility since splice cassette "B" in the NL1 extracellular domain contains an asparagine residue that is a target site for glycosylation (Hoffman et al., 2004). This N-linked glycan determines binding to Neurexins and correlates with excitatory versus inhibitory synapse association (Boucard et al., 2005; Chih et al., 2006). Future studies in heterologous systems may be suited to examine which mechanisms determine which Neuroligin pairs are permitted to form, while studies in neurons can further examine how these mechanisms are regulated and differ between neuron types.

It is conceivable that different sets of neurons posses distinct ensembles of Neuroligin dimers. The possibility of neuron- or region-specific differences demands the expansion of the study of dimer composition by systematically examining several neuron types. Distinct dimer ensembles may additionally arise from quantitative differences in the prevalence of specific dimer species. To examine this possibility, future studies would have to provide quantitative readouts in terms of the prevalence of dimer combinations for each preparation. The information from such analyses might prove invaluable to relate specific Neuroligin dimers with specific synapses by making correlations between the prevalence of dimer species and synapse type in each preparation. In conjunction with immunohistochemical analyses of the colocalization of Neuroligin paralogues, these studies may allow us to 
revisit the models of Neuroligins at the synapse, ultimately, by assigning dimeric species to corresponding synapse types.

\subsection{Neuroligin dimers: assembly and signaling}

The significance of having diverse populations of Neuroligin dimers stems from the surprising finding that, while the members of the Neuroligin family are well conserved (Ichtchenko et al., 1996), they have strikingly different functional properties (Graf et al., 2004; Chih et al., 2005; Levinson et al., 2005; Chubykin et al., 2007; Aramuni et al., submitted for publication). As the dimer appears to be the functional Neuroligin unit, the critical question is how heterodimerization blends the properties of the paralogues into the coherent properties of the dimer.

Implicated in transsynaptic adhesion, the properties of Neuroligin dimers will affect both presynaptic and postsynaptic machineries. Neurexins, the presynaptic binding partners of Neuroligins, are highly diverse. The complex gene organization of the Neurexin family, with three paralogous genes containing two alternaive promoter sites and up o five canonical alternative splice sites, can potentially result in over one-thousand unique gene products (Ullrich et al., 1995; reviewed by Missler et al., 1998). Though far less is known about the functional consequences of Neurexin diversity, it is well documented that different Neuroligins have different preferences for Neurexin isoforms (Ichtchenko et al., 1996; Boucard et al., 2005; Graf et al., 2006; Chih et al., 2006; Comoletti et al., 2006; Kang et al., 2007). Thus Neuroligin dimer composition would influence the selectivity for Neurexin binding.

Structural studies have indicated that each Neuroligin molecule in a dimer binds to a Neurexin molecule independently (Comoletti et al., 2007 Araç et al., 2007; Chen et al., 2007; Fabrichni et al., 2007; Koehnke et al., 2008). This implies that a given postsynaptic Neuroligin dimer would select the Neurexin isoforms clustered on the presynaptic terminal. As Neurexins bind to active zone proteins (Hata et al., 1993; Hata et al., 1996) and have been shown to recruit components of the presynaptic apparatus (Scheiffele et al., 2000; Dean et al., 2003), the postsynaptic Neuroligin dimer species could effectively determine properties of the active zone of trans- 
mitter release. This would constitute retrograde synaptic signaling from the postsynaptic to the presynaptic component.

Alternatively, the presynaptic Neurexin isoforms arranged on the protein scaffold of the presynaptic terminal could dictate which Neuroligin paralogues cluster at sites of contact with the postsynaptic cell. Conceivably, if Neuroligin dimer assembly were to take place at the neuronal surface at sites of presynaptic terminal contact, a mechanism involving selective Neurexin recruitment of Neuroligins could dictate dimer combinations by way of a herding mechanism; specific Neuroligin paralogues would be selectively recruited by presynaptic Neurexins. Brought into close proximity in the vicinity of a nascent postsynapse, selected Neuroligin paralogues would subsequently dimerize. The result would be specific Neuroligin dimer pairs mirroring the respective Neurexin isoforms that recruited them. This would effectively constitute anterograde synaptic signaling where the presynapse would dictate properties of the postsynapse.

A critical point in the question of directionality in the transsynaptic signaling of the Neuroligin-Neurexin adhesion complex is the site of Neuroligin dimer assembly. Do dimers form at the synapse or do they arrive at the synapse preformed? Equivalently, does the postsynaptic cell alone determine the dimer composition or is the presynaptic cell also involved? Using Neuroligin mutants that are deficient in their capacity to dimerize, insight was provided into the cellular compartment that accommodates dimer formation. Introduction of the monomeric mutation into Neuroligins expressed in cell-lines caused a change in their electrophoretic band pattern. Unmutated Neuroligins in heterologous systems typically have a band pattern consisting of a fast-migrating band doublet and a slower migrating diffuse band. Monomeric Neuroligin mutants exhibited an elecrophoretic band pattern where the low-mobility, diffuse band is diminished (Figure $24 \mathrm{~B}$ ). This band was found to represent a glycosylated species that were susceptible to PNGase F but resistant to Endoglycosidase $\mathrm{H}$ treatments (Figure 26). This observation is indicative of the presence of mature $\mathrm{N}$-glycans whose formation is catalized by enzymes residing in the trans-Golgi network of the secretory pathway. The presence of mature $\mathrm{N}$ glycans on a protein can be seen as a molecular signature of the trans-Golgi network that marks proteins that have traversed this compartment on their way through the secretory pathway towards the cell surface. It discriminates them from 
proteins that reside in the early secretory compartments of the endoplasmatic reticulum (ER) and the cis-cisternae of the Golgi apparatus which are not maturely glycosylated. It thus appears that Neuroligin monomers are prevented from reaching the trans-Golgi network and, consequently, the cell surface.

Neuroligin monomers show deficient trafficking to the plasma membrane. However, increasing the expression level of the mutants led to an increase in the ratio of mature to immature protein detected (data not shown). This is an indication that Neuroligin monomers are not inherently incapable of trafficking to the plasma membrane, rather they are actively retained by an in trans saturatable factor. In neurons, expression of monomeric Neuroligin mutants was toxic and led to the accumulation of the protein in somata of cellular remains of transfected neurons (data not shown). As monomers appear to be retained, it seems likely that toxicity was mediated by ER stress. Healthy neurons expressing the mutants on their surface were not observed. It thus appears that a very effective retention mechanism exists in neurons that allows Neuroligins to reach the postsynaptic membrane only after they have formed dimers.

Precedents of quality control mechanisms in the assembly of membrane protein complexes, most notably of ionotropic receptors and ion channels, are abundant in the literature. GABA receptors traffic to the plasma membrane only after proper assembly into pentamers in the ER (reviewed by Kittler et al., 2002). Mechanisms have been identified for glutamate receptors (reviewed by Fleck, 2006; Greger et al., 2007) and potassium channels (reviewed by Ma \& Jan, 2002) whereby ER retention signals mediate the association of single subunits with resident proteins of the secretory pathway.

Following the notion of a possible membrane complex quality control mechanism acting on Neuroligin dimer assembly, the primary sequence of Neuroligins was examined to identify conserved putative retention signals. The transmembrane domain, highly conserved in all four Neuroligins, exhibits primary sequence properties that hint at its involvement in putative quality control mechanisms. A conserved asparagine residue appears in the middle of the transmembrane sequence (Figure $26 \mathrm{~B})$, placing a residue with a polar side-chain within the aliphatic phase of the lipid bilayer. Exposure of the asparagine dipole to the hydrophobic environment of 
the membrane would be thermodynamically unfavorable. The charge would thus be expected to be neutralized through interaction with other charges in the aliphatic phase.

Examples of charged groups of transmembrane domains being involved in secretory pathway retention or retrieval mechanisms can be found in yeast. Sec12p, Sec63p and Sec71p, components of secretory pathway sorting in Saccharomyces cerevise, are actively retained in the early secretory pathway. The mechanisms by which this is achieved involve Rer1p, a tetra-spanning membrane protein that cycles between the cis-Golgi compartment and the ER (Sato et al., 1996, Sato et al., 1997). Retrieval is shown to be mediated by an interaction of Rer1p with polar residues of the transmembrane domains of these Sec proteins leading to their retrieval from cis cisternae to the ER (Sato et al., 2001; Sato et al., 2003).

An indication that a similar mechanism may be involved in the retention of Neuroligin monomers in mammalian cells comes from the observation that substitution of the transmembrane domain asparagine residue by an aliphatic residue in monomeric Neuroligin mutants resulted in the reversal of the trafficking deficiency phenotype. The ratio of maturely to immaturely glycosylated protein was similar in unmutated HA-NL2 and HA-monoNL2/N701L, while it was greatly reduced in HAmonoNL2 (Figure 26). This observation substantiates the notion that Neuroligin monomers are actively retained intracellularly and provides mechanistic insight implicating a conserved asparagine residue in the transmembrane domain of Neuroligins.

A mammalian orthologue of yeast Rer1p (Füllekrug et al., 1997) was recently shown to be implicated in a membrane protein complex quality control mechanism. Mammalian Rer1 was identified as an in cis interaction partner of Pen2, a dispanning transmembrane protein. Pen2 is one of four distinct transmembrane protein constituents of the $\gamma$-secretase protease complex which traffics to the surface only upon full assembly (reviewed by Kaether et al., 2006). Rer1 was shown to retain uncomplexed Pen2 in the early secretory pathway via an asparagine residue in its first transmembrane domain.

The obvious functional and structural analogies of Neuroligins with Pen2 in terms of monomer retention raise the possibility that Neuroligins are retained via a simi- 
lar mechanism, being potential substrates of a resident component of the early secretory pathway with properties similar to Rer1. These analogies could provide the basis of a hypothetical mechanistic model for Neuroligin dimerization (Schema 7). Synthesis and integration of Neuroligin monomers into the membranes of the ER would bring the transmembrane asparagine within the hydrophobic environment of the lipid bilayer. A protein resident to the early secretory pathway, capable of interacting with polar transmembrane residues, could stabilize the charge of the asparagine side-chain and lead to complex formation. Neuroligin monomers in complex with the resident protein would thus be retained in the early secretory pathway. However, asparagine-containing helices within a lipid environment have been shown to spontaneously dimerize in order to neutralize their side-chain dipoles (Choma et al., 2000; Zhou et al., 2000). Neuroligin transmembrane domains would thus be expected to tend to form dimers. As the extracellular domain of Neuroligins

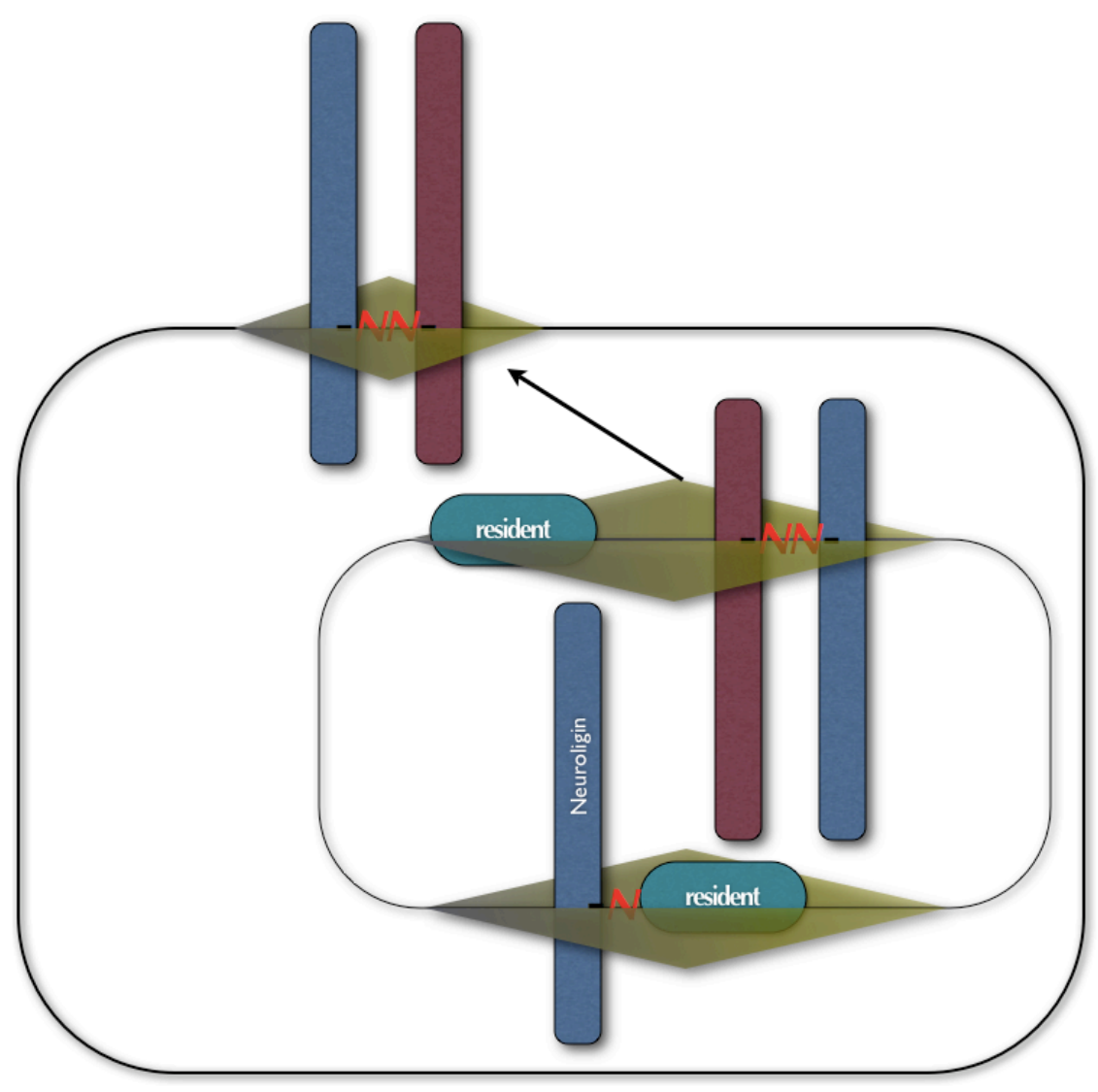

Schema 7: Model of Neuroligin dimerization and quality control. Neuroligins synthesized in the ER bind via their transmembrane asparagine to a protein resident in the early secretory pathway, leading to Neuroligin monomer retention. Dimerization of Neuroligins by their extracellular domains leads their mutual transmembrane asparagine side chains to couple, thus being released from the resident protein. Dimers are then "free" to traffic towards the cell surface. 
alone additionally mediates dimerization, Neuroligin pairing appears highly favorable. The formation of Neuroligin dimers in the early secretory pathway would mask their transmembrane asparagines and effectively compete with the resident binding partner. According to the proposed model, dimer formation would lead to the release of Neuroligins from the resident protein, freeing them for further transport to the later compartments of the secretory pathway to undergo mature glycosylation and, eventually be presented on the cell surface.

There are several implications of the proposed model on our current understanding of Neuroligin function. Firstly, it regards the Neuroligin dimer as a conical membrane protein complex together with others like $\mathrm{Y}$-secretase and ion channels. The model incorporates this classification by distinguishing Neuroligin dimerization from that of receptor dimerization, regarding it as a structural feature rather than a functional mode of activation. It further implies that dimerization may not solely be mediated by the Neuroligin extracellular domains, but additionally by the polar epitopes of the transmembrane domains. Most importantly, it proposes that dimer formation takes place in the early secretory pathway and that the postsynaptic cell actively prevents Neuroligin monomers from reaching the surface, excluding the presynaptic cell from determining the composition of dimers. The model thus proposes that adhesion signaling through the transsynaptic Neuroligin-Neurexin complex is retrograde in nature with the postsynaptic cell determining dimer species that the presynaptic cell responds to.

\subsection{Postsynaptic functions of the Neuroligin family: re- ceptors, scaffolds and regulators}

Despite strong evidence of an integral role of Neuroligins, in conjunction with Neurexins, in determining the presynaptic component of the synapse, mouse genetics evidence clearly indicates that the most prominent of Neuroligin functions involves the postsynaptic apparatus (Varoqueaux et al., 2006). After their initial identification as Neurexin binding partners (Ichtchenko et al., 1995), Neuroligins were soon found to interact with the abundant postsynaptic scaffolding protein PSD-95 (Irie et al., 1997; Bolliger et al., 2001). This interaction was found to be 
mediated by a conserved five-residue C-terminal consensus motif which binds PDZ domains on PSD-95. Soon after, other PDZ domain-containing proteins, such as MAGIs, SAP102 and SHANK, were added to the list of Neuroligin interaction partners (Hirao et al., 1998; Kurschner et al., 1998; Meyer et al., 2004). As PDZ domains are typical modules of proteins involved in junctional scaffolds, and a prominent feature of the Neuroligin cytoplasmic domain is to interact with them, there appears to be a tight association between Neuroligin function and scaffolding proteins.

PDZ domain-containing proteins like PSD-95 are components of every glutamatergic postsynapse. However, these proteins are not prominent components of inhibitory postsynapses. In addition to the PDZ-binding motif of the Neuroligin cytoplasmic domain, evidence is presented herein of another conserved motif which allows Neuroligins to interact with Gephyrin, the major scaffolding protein of GABAergic and glycinergic postsynapses (Figures 11 and 14; see below). In fact, the PDZ-binding motif together with the Gephyrin-binding motif comprise virtually all of the conserved sequence clusters of the cytoplasmic domains of the Neuroligin paralogues (Schema 4). This indicates that, aside from paralogue specific functions, scaffolding protein binding is a core feature of Neuroligins.

In addition to PDZ domain-containing proteins and Gephyrin, yeast-two-hybrid data presented herein imply that Dystrophin-Related Protein 2 (DRP2) may also have the capacity to interact with the cytoplasmic domains of all four paralogues. Though the binding site was not determined, combined mutations in the vicinity of the Gephyrin-binding motif (P768A/Y770A) and a C-terminal truncation (P798stop) encompassing the proline-rich stretch and the PDZ-binding motif of NL2 were necessary to abolish binding (Table 3). DRP2 has been shown to exhibit a punctate distribution in the brain corresponding to a subset of synaptic contacts (Roberts and Sheng, 2000). However in the present study, DRP2-specific antibodies raised against two distinct epitopes failed to detect DRP2 in adult rat brain homogenates, though they did detect DRP2 in lysates from transfected fibroblasts. The source of the discrepancy regarding expression of DRP2 in the central nervous system between the report of Roberts and Sheng (2000) and the present study is unclear. However, as DRP2 was identified herein as a yeast-two-hybrid 
prey clone from a neonatal rat cDNA library, it is suggested that gene products of DRP2 are indeed present in the brain.

In the case that DRP2 is confirmed to be a common interaction partner of Neuroligins in the brain, this would be consistent with the notion of Neuroligins possessing core binding properties for postsynaptic scaffolding proteins. DRP2 has an identified scaffolding role in Schwan cells of the peripheral nervous system (Sherman et al., 2001). Additionally, as a protein homologous to Dystrophin (Roberts et al., 1996), DRP2 is expected to function as a scaffolding molecule. Dystrophin itself is a major scaffold of junctional complexes in epithelial, muscle and nervous tissue (reviewed by Sgambato \& Brancaccio, 2005). In fact, in light of the postsynaptic localization of Dystrophin, a potential interaction between Dystrophin and the Neuroligins would warrant future study.

Together, confirmed and putative cytosolic interaction partners that are common to the four Neuroligins all have roles as scaffolding proteins. As Neuroligins are functionally regarded as adhesion molecules, a broad model of junctional complexes where adhesion and scaffolding molecules in succession structurally link extracellular elements to cytosolic components of the cellular machinery can be applied. Projecting this notion to the synapse, all Neuroligins have the protein-binding properties to bridge the contacting presynaptic terminal and the postsynaptic scaffold of both excitatory and inhibitory synapses.

Postsynaptic scaffolds form the matrix onto which neurotransmitter receptors reside. PSD-95 was shown to interact with NMDA-type glutamate receptors via its first and second PDZ domains, while being still able to bind Neuroligins via its third PDZ domain (Irie et al., 1997). Synaptic Scaffolding molecule (S-SCAM or MAGI2) can similarly bind both Neuroligins and glutamate receptors, NMDA-types directly (Hirao 1998; lida et al., 2004) and AMPA-types via Stargazin (Deng et al., 2006). At inhibitory synapses, the scaffolding protein Gephyrin can directly bind to (Kirsch et al., 1991; Meyer et al., 1995) and cluster glycine receptors at postsynaptic sites (Kirsch et al., 1993; Feng et al., 1998). It can additionally associate with some types of $\mathrm{GABA}_{A}$ receptor subunits (Tretter et al., 2008) and is required for the synaptic clustering of the majority of $\mathrm{GABA}_{\mathrm{A}}$ receptors (Essrich et al., 1999, Kneussel et al., 1999). There may also be a more direct link between Neuroligins 
and $\mathrm{GABA}_{A}$ receptors (Dong et al., 2007). Equivalent phenomena involving Neuroligins and PDZ-containing proteins may additionally be involved in cholinergic synapse formation (Temburni et al., 2004; Conroy et al., 2007; Ross et al., 2007; see below). Thus the link that Neuroligins mediate on the postsynaptic side can cover the functional circuit of transmitter release to transmitter receptors.

The functional properties of Neuroligins at the postsynapse however appear more complex than those of a structural component of the synaptic junction. The first studies into the function of Neuroligins indicated that they are involved in synaptogenesis by way of recruiting presynaptic terminals to sites of Neuroligin clusters (Scheiffele et al., 2000) and inducing presynaptic differentiation (Dean et al., 2003). Consequently, subsequent research into Neuroligin function concentrated on the mechanism by which Neuroligins are themselves targeted to sites of synaptic contacts.

lida et al. (2004) propose a mechanism by which S-SCAM is first recruited to postsynaptic sites by $\beta$-Catenin to subsequently recruit NL1 and PSD-95. However, interpretation of experiments therein, where PDZ domain deletion mutants are introduced into neurons, are made liable by the promiscuity of PDZ domains (Meyer et al., 2004). Additionally, Dresbach et al. (2004) showed that NL1 targeting to synapses is independent of its PDZ-binding motif and of any sequences downstream of the membrane-proximal region of the Neuroligin cytoplasmic domain. $A$ recent study similarly found the NL1 PDZ-binding motif to be redundant for NL1 function at cholinergic synapses (Conroy et al., 2007). Both these studies, however, face the caveat of introducing cytosolic mutations in neurons of a wild-type background. In light of the data herein on Neuroligin dimerization in neurons, it becomes plausible that exogenous Neuroligin mutants introduced into wild-type neurons can dimerize with endogenous unmutated Neuroligins. This would produce a dimer with a tag from exogenous Neuroligin mutants and a functional cytoplasmic domain from the endogenous molecule. In this configuration, the effects of a lossof-function mutation, such as deletion of the PDZ-binding domain, would be masked as tagged complexes would contain unmutated motifs. This interpretation is supported by observations in the present study, indicating that all HA-NL2 cytosolic mutations examined in wild-type neurons showed no effect in their ability to cluster Gephyrin (data not shown), while loss-of-function effects were observed in 
neurons from a NL2-deletion background (Figure 18). Interestingly, the observation that deletion of the membrane-proximal region of the NL1 cytoplasmic domain did interfere with synaptic targeting (Dresbach et al., 2004) may be interpreted in view of the importance of the transmembrane domain in Neuroligin trafficking presented herein and possible disruption of this mechanism by membrane proximal deletions.

The question of synaptic targeting and recruitment as functions of Neuroligins remains open, though new evidence points to the existence of preformed clusters of Neuroligins with PSD-95 which are mobile and unassociated with a presynaptic terminal (Gerrow et al., 2006). The role of the presynaptic terminal thus becomes more prominent as the question of synaptic targeting translates into the joining of preformed postsynaptic clusters with a contacting axon terminal. This notion is especially appealing in view of evidence suggesting that Neuroligin synapse specificity between excitatory and inhibitory synapses can be determined by splicedependent interactions with presynaptic Neurexins (Boucard et al., 2005; Chih et al., 2006; Graf et al., 2006; Kang et al., 2007). In fact, one of the most prominent functions of Neuroligins in vivo and in vitro appears to be their selective effects on excitatory or inhibitory neurotransmission.

Mice lacking NL1 exhibit a reduction in NMDA transmission by approximately $50 \%$ in the hippocampus, while AMPA and inhibitory transmission remain unchanged (Chubykin et al., 2007). Conversely, mice lacking NL2 show deficits in inhibitory transmission in the somatosensory cortex (Chubykin et al., 2007) and a drastic reduction of both GABAergic and glycinergic transmission in the brain stem without effects in excitatory transmission (Aramuni et al., submitted for publication). These in vivo data complement studies where the synaptogenic effects of Neuroligins observed in culture also distribute accordingly; NL1 favors excitatory synapse formation, while NL2 favors inhibitory synapse formation (Graf et al., 2004; Chih et al., 2005; Levinson et al., 2005; Chubykin et al., 2007). These observations have led to the hypothesis that the two paralogues are involved in the establishment of balance between excitation and inhibition in the central nervous system (Prange et al., 2004; Cline, 2005; Levinson \& El-Husseini, 2005). 
Despite the convergence of data on the specificity of NL1 for excitatory synapses and NL2 for inhibitory synapses, a mechanistic explanation is lacking. The specificity suggested to arise from extracellular interactions with Neurexins dictated by splice site B unique to NL1 has been contested (Chubykin et al., 2007). On the cytosolic side, promiscuous interactions of all Neuroligin paralogues with both excitatory and inhibitory scaffolds is even more confounding. As noted previously, all Neuroligins contain a PDZ-binding motif and are expected to interact with excitatory scaffolding proteins like PSD-95. Moreover, all Neuroligins contain a Gephyrin-binding motif (Figure 14 and Schema 4) and can associate with Gephyrin membrane microaggregates (Figure 15). It thus appears that all Neuroligins have the potential to associate with either scaffold, although in vivo they colocalize preferentially with one of the two (Song et al., 1999; Varoqueaux et al., 2004).

Looking closely at studies where the normal balance of Neuroligin paralogues or of scaffolding proteins is perturbed, one can observe a manifestation of the Neuroligin structural promiscuity in neurons. Reduction of expression levels of single Neuroligin paralogues in cultured neurons by RNA interference showed that knock-down of any single Neuroligin paralogue resulted in the reduction of both glutamatergic and GABAergic innervation (Chih et al., 2005). Equivalently, overexpression of any single Neuroligin paralogue resulted in the increase of both excitatory and inhibitory innervation (Levinson et al., 2005); albeit overexpressed NL2 was more effective at recruiting inhibitory contacts (Chih et al., 2005). Additionally, though NL2 is not seen to colocalize with PSD-95 physiologically, overexpression of PSD-95 leads to its co-clustering with NL2 (Graf et al., 2004, Levinson et al., 2005). These data clearly show that, while all Neuroligin paralogues have properties that allow them to associate with either excitatory or inhibitory synapses, Neuroligins exhibit specificity regarding synapse type when physiological levels of expression are unperturbed.

The dependence of the in vivo specificity on expression levels along with the promiscuous structural features of Neuroligins indicate that under physiological circumstances, regulatory mechanisms function to ensure specificity. As it has been suggested that excitatory and inhibitory scaffolds compete for Neuroligin binding (Levinson \& El-Husseini, 2005), differential regulation of the interaction of Neuroligin paralogues with one of the scaffolding proteins would be in line with this hy- 
pothesis. Interestingly, an exception to the otherwise conserved nature of the Gephyrin-binding motif pertains to a substitution of the serine residue at position 10 of the consensus sequence by alanine only in NL2 (Figure $14 \mathrm{~A}$ ). This serine residue is predicted to be the target of phosphorylation due to the arginine residues found immediately N-terminal (Songyang et al., 1994). Phosphomemetic mutation of serine 802 in NL1 to glutamic acid disrupted binding to Gephyrin in yeast (Figure $14 \mathrm{~B}$ ). Though phosphorylation of Neuroligins remains to be verified in vivo, the experimental evidence indicates that phosphorylation of the Gephyrinbinding motif serine in NL1, NL3 and NL4 may result in the loss of their capacity to interact with Gephyrin. As NL2 has an alanine residue on the equivalent position, it would be exempt from this regulatory mechanism and be able to bind Gephyrin constitutively.

Potential regulation of Gephyrin-binding in Neuroligins 1, 3 and 4 is in good agreement with observations that these paralogues are predominantly associated with excitatory synapses (Graf et al., 2004). In conjunction with data showing that NMDA receptor-mediated activation of $\mathrm{Ca}^{2+} /$ calmodulin-dependent protein Kinase II (CaMKII) is required for NL1 to exert its effect on excitatory synapses in neuron culture assays (Chubykin et al., 2007), a model of activity-dependent scaffold selectivity for Neuroligins can be proposed. At the initial stages of synapse formation, Neuroligins associate promiscuously with either excitatory or inhibitory scaffolds. This is in accordance with observations of mismatched or mixed scaffolds in nascent synaptic contacts in hippocampal (Rao et al., 2000; Anderson et al., 2004) and cerebellar cultures (Studler et al., 2002). After the presynaptic terminal becomes release-competent and the synapse is activated, glutamate release would trigger the activity-dependent activation of a postsynaptic protein kinase, as in the case of the NMDA-mediated cascade leading to CaMKII activation. Neuroligins 1, 3 or 4 at the site of active glutamatergic terminal contact would be targets for Gephyrin-binding domain phosphorylation. These excitatory Neuroligins would loose their affinity for Gephyrin which would no longer remain associated. In that way, activity-dependent phosphorylation could favor the association of excitatory Neuroligins with PDZ domain-containing scaffolds.

The putative mechanism described above cannot be the sole determinant of proper transmitter to receptor apposition, as chronic activity blockade of transmis- 
sion does not ultimately lead to the prevalence of mismatched synapses (Harms et al., 2005), nor are mismatched synapses more prevalent in mice incapable of fast synaptic transmission (Varoqueaux et al., 2002). However, it may be one of the mechanisms that regulate the specificity exhibited by Neuroligin paralogues for excitatory versus inhibitory postsynapses.

The fact that Neuroligins all potentially bind both inhibitory and excitatory scaffolds in a regulated manner increases the versatility of Neuroligins to participate in more refined synapse-specific processes. In light of the fact that Neuroligins are shown to form heterodimers, this becomes a crucial point in order for paralogue incompatibilities to be avoided. An exemplary case is the detection of a minor fraction of $\mathrm{NL} 1 / 2$ heterodimers in hippocampal neurons in culture (Figure $25 \mathrm{~A}$ and $\mathrm{B}$ ). If synapse specificity in NL1 and NL2 was rigid, then this heterodimer would be incompatible for both synapse types. Alternatively, if specificity is regulated on a caseby-case basis at the synapse, it is conceivable that this heterodimeric species could be accommodated for at certain synapse types. In agreement with this notion is the observation by Levinson et al. (2005) who report that a small fraction of endogenous NL1 clusters associate with inhibitory synapse markers in hippocampal neurons in culture. It is plausible that this fraction of NL1 represents the fraction biochemically identified to heterodimerize with NL2. It thus appears that despite the extensive synapse specificity observed for Neuroligin paralogues, there is room for their association at diverse synapse types.

The allowance for diversity of paralogue synapse specificity becomes especially interesting when one contemplates on the possible paralogue-specific functions that Neuroligins may posses. Apart from the core cytosolic interaction partners of PDZ-containing proteins, Gephyrin and potentially DRP2, there are other putative binding partners that do exhibit paralogue specificity. The list of candidate cytosolic interaction partners of NL2 from the yeast-two-hybrid screen was examined for potential paralogue selectivity by cross-screening using Neuroligin 1, 3 and 4 cytosolic bait constructs. The results from this study were surprising in the extent to which candidate interactors distributed into overlapping combinations of paralogue binding (Schema 5). 
Aside from the group of core interaction partners, NL2 putative cytosolic interactors can further be grouped by their paralogue specificity into NL2/3-, NL2/4-, NL1/ 2/4-, NL2/3/4-specific interactors and interactors specific for NL2 alone. As the approach taken was inherently biased for NL2, since all candidates were preselected for NL2 binding, it is expected that other groups of Neuroligin cytosolic interactors with paralogue-specific preferences excluding NL2 may exist. These observations, in conjunction with the detection of Neuroligin dimers with diverse paralogue compositions, imply the existence of cytosolic interaction partners that show preference to specific dimer species. A protein that can interact with both cytosolic tails of a Neuroligin dimer would have increased avidity for interacting with that particular dimer over a protein that can bind only one of the two cytosolic tails. A model taking into account such an avidity-based selectivity mechanism would allow Neuroligin dimer species to recruit specific auxiliary interaction partners in conjunction to being tethered with their core binding partners of the synaptic scaffolds (Schema 8). Such an arrangement would serve to confer dimer-specific properties to the synapse type that a given dimer associates with.

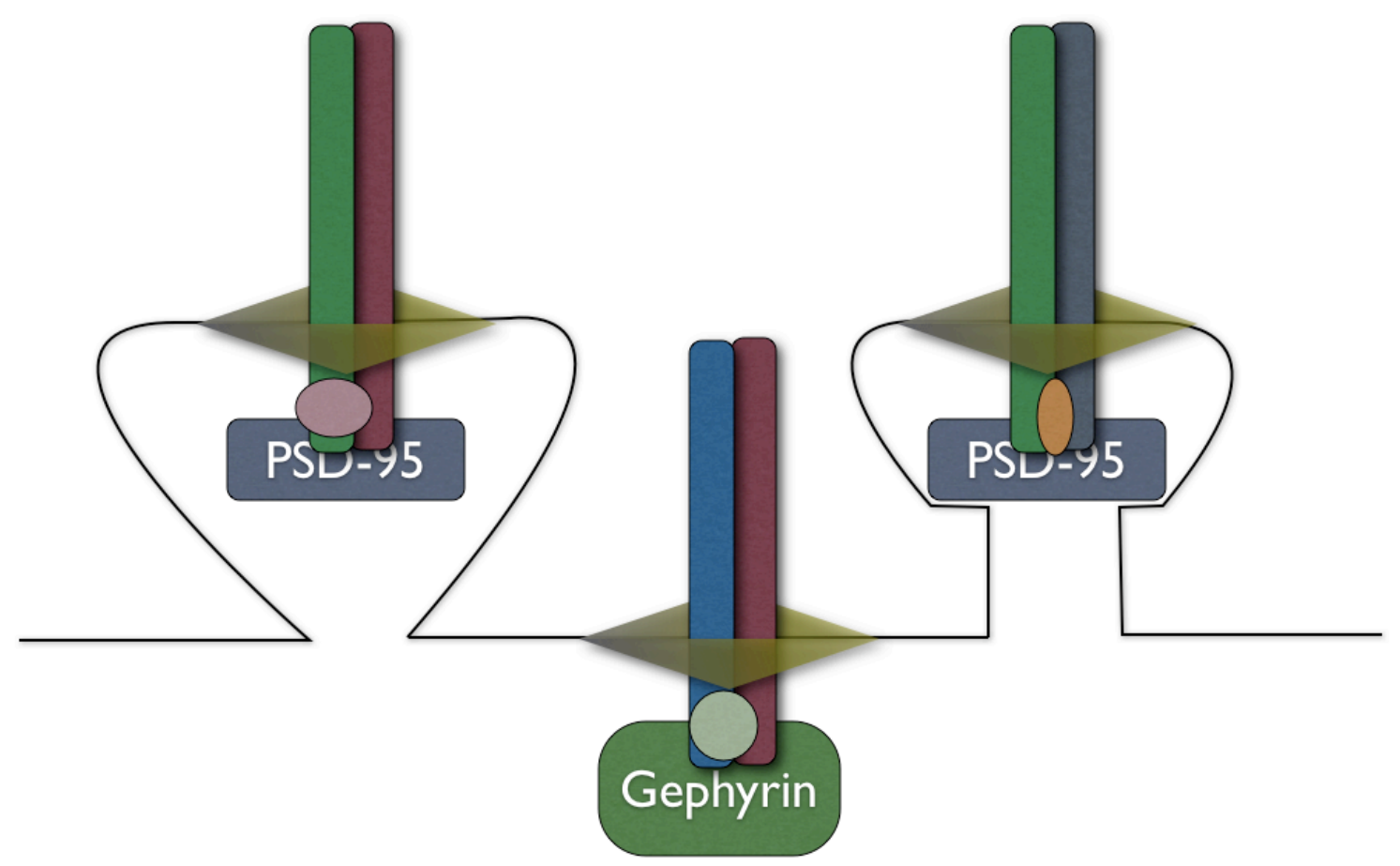

Schema 8: Model of dimer-specific auxiliary Neuroligin interactors. Neuroligin dimers invariably bind to scaffolding proteins such as PSD-95 and Gephyrin as core cytoplasmic interactors. Interaction partners specific to a dimer species are recruited as auxiliary components that may regulate properties of the postsynapse differentially. 
Drawing the parallel between the paralogue specificity exhibited by putative Neuroligin cytosolic interactors in yeast and Neuroligin dimer species detected in hippocampal neurons, several correlations may be pointed out. The majority of putative interaction partners identified showed exclusivity to the NL2 cytoplasmic domain over the other paralogues. This may imply that there are protein interactors that are selectively accommodated by putative NL2 homodimers. This correlates with the biochemical evidence showing that heteromeric NL2-containing dimer species are rarely detected.

This group is dominated by proteins known to be associated with the actin cytoskeleton or involved in regulating its dynamics, such as a-Actinin (reviewed by Jockusch et al., 2004), Abi-I (Proepper et al., 2007), $\beta$-Catenin (reviewed by Salinas \& Price, 2005), FBP11 (reviewed by Wallar \& Alberts, 2003), Kalirin (Ma et al., 2003), Profillin II and VASP (reviewed in Krause et al., 2003). Interestingly, the latter two have been shown to interact with Gephyrin and localize to inhibitory synapses (Mammoto et al., 1998; Giesemann et al., 2003; Bausen et al., 2005) as is the case for NL2, though this finding was not reproduced in yeast-two-hybrid screens presented herein (Table 2). The association of NL2 with the actin cytoskeleton is further substantiated by the reported functional features relating to actin dynamics of the proline-rich stretch (reviewed by Holt \& Koffer, 2001) that dominates the NL2 cytoplasmic domain, and by the effect of NL2 overexpression in inducing filopodial protrusions in fibroblasts and neurons reported herein and previously (Chubykin et al., 2007). These properties of NL2 have obvious potential implications for the morphological characteristics of the postsynaptic compartment.

Another interesting NL2-specific putative interaction partner is EB1, a protein associated with microtubule dynamics (reviewed by Vaughan, 2005). The putative interaction with NL2 becomes especially interesting in light of the potential role of EB1 and its binding partner APC in nicotinic receptor clustering at cholinergic synapses (Temburni et al., 2004), where Neuroligins have been reported to participate in postsynaptic assembly (Conroy et al., 2007; Ross et al., 2007). Though these studies implicated NL1, the paralogue specificity of the antibody employed was not directly examined, though it appears that a NL2 orthologue is absent from the chick, on which the studies were performed. An additional conceptual link to NL2 in nicotinic synapses is that APC, a critical clustering component of nicotinic recep- 
tors binds Asef, the closest homologue of Collybistin. These two proteins share properties of SH3-mediated inhibition of $\mathrm{PH}$ domain-mediated membrane association, processes in which NL2 is suggested to be involved (see § 4.4).

Among the group of proteins with mixed paralogue specificities as detected in the yeast-two-hybrid assays, NL2/4 specific interactors were most abundant. This may reflect a common feature in the two cytoplasmic domain sequences which exhibit non-homologous proline-rich regions. As detection of NL4 was not achieved in hippocampal cultures, there is no information regarding possible NL2/4 heterodimers. The yeast-two-hybrid data, though, imply that this could be a prevalent species. There were no candidates identified with selectivity for $\mathrm{NL} 1 / 2$, while the ubiquitin ligase NEDD4 was the only candidate selective for NL1/2/4. The lack of a specific overlap of NL1/2 binding partners possibly reflects the prevailing segregation of these paralogues into different synapse types. In the case of NL2/3, there is a limited overlap of interactors, however NL2/3 dimeric species were not detected in hippocampal cultures. It will be interesting to test for their existence in neurons from other brain regions to see if there may be region-specific dimerization which may give rise to region-specific recruitment of NL2/3 interactors.

Taken together, there is ample evidence that Neuroligins, through a combination of versatility in scaffold association and specificity towards signaling and regulatory proteins, have the capacity to endow postsynapses with diverse properties. Through possible activity-dependent regulation of scaffold association, Neuroligins may attain postsynaptic specificity on a synapse-by-synapse basis. Dimer selectivity of synapse type may combine with dimer species-specific interactors to regulate postsynapse morphology and signaling characteristics. This wide range of capabilities greatly increases the spectrum of Neuroligin function at the postsynapse.

\subsection{Mechanistic role of Neuroligin 2 at the inhibitory postsynapse}

The first studies identifying synaptic adhesion molecules directly involved in synapse formation concerned only excitatory synapses. Both NL1 and NL2 were first reported to be involved in excitatory synapse formation (Scheiffele et al., 2000). 
Subsequently, SynCAMs (Sara et al., 2005), Netrin G Ligands (Kim et al., 2006) and SALMs (Ko et al., 2006) were all shown to have similar in vitro properties in inducing synapse formation, reportedly exclusively of glutamatergic synapses. The first indications that Neuroligins may also have a function at inhibitory synapses came from the strictly inhibitory synapse localization of endogenous NL2 observed by Varoqueaux et al. (2004). Graf et al. (2004) further provided evidence of a functional role for NL2 at inhibitory synapses by showing that Neurexins could induce GABAergic postsynaptic differentiation via NL2. Deletion of multiple Neuroligins and specific deletion of NL2 confirmed its selective function at inhibitory synapses (Varoqueaux et al., 2006; Chubykin et al., 2007; Aramuni et al., submitted for publication).

In retrospect, based on our current understanding that Neuroligins can also be aNeurexin ligands (Boucard et al., 2005), the first experimental data revealing a function for the Neuroligin-Neurexin complex at inhibitory synapses came from an a-Neurexin triple deletion mutant mouse which form only half the number of symmetric synapses compared to wild-type animals (Missler et al., 2003). Subsequently, the role of a-Neurexins preferentially at inhibitory versus excitatory synapses was further substantiated by in vitro co-culture of neurons together with aNeurexin transfected fibroblast (Kang et al., 2007).

Currently, the NL2-a-Neurexin system is the only documented adhesion system which appears to be functional preferentially at inhibitory synapses. Despite the wealth of data supporting this notion, there is no direct structural or mechanistic evidence which can interpret -or even accommodate for- the functionality at inhibitory synapses. In the work presented herein, a novel interaction of the Neuroligin cytoplasmic domain with the protein Gephyrin is documented. As Gephyrin is the central scaffolding protein of inhibitory synapses, this interaction provides a critical structural framework for Neuroligins at inhibitory synapses.

Gephyrin was identified as a putative cytosolic NL2 interaction partner through yeast-two-hybrid screening of rat brain cDNA. Biochemical evidence that NL2 and Gephyrin are found in complex in vivo comes from the detection of adducts containing both NL2 and Gephyrin in crosslinked brain preparations (Figure 13 A). An indication that NL2 and Gephyrin are not indirectly linked in a complex by unidenti- 
fied synaptic proteins comes from data showing that NL2-Gephyrin complexes can be similarly detected in fibroblasts exogenously expressing the two proteins (Figure $13 \mathrm{~B}$ ). The fact that this interaction was observed in yeast in the absence of other mammalian proteins further substantiates the notion that the NL2-Gephyrin interaction is direct.

To directly address the question of direct versus indirect interaction of NL2 with Gephyrin, attempts were made to observe complex formation in binding assays using an assortment of recombinant soluble forms of the NL2 cytoplasmic domain. Most of these approaches yielded inconclusive results due to a combination of very inefficient complex formation and high background levels. Nonetheless, marginally detectable specific complex formation was observed using purified Gephyrin preparations. Though GST-NL2CD formed a non-stoichiometric complex with purified Gephyrin as observed using a pulldown approach, the NL2CD/P768A/ Y770A mutation, shown to abolish the interaction in yeast, did not (Ingo Paarman, Frankfurt; personal communication). This, together with the sum of indirect evidence, strongly argues in favor of direct NL2-Gephyrin complex formation.

Two independent clones of Gephyrin were identified as putative Neuroligin binding partners in yeast; one corresponded to the full-length protein and one to a 286-768 Gephyrin fragment corresponding roughly to the Gephyrin E-domain. A fusion construct of GFP with this Gephyrin fragment confirmed that it is sufficient to mediate NL2 complex formation in cell biological assays (data not shown). The Gephyrin Edomain contains a substantial amount of additional binding sites as it has previously been shown to mediate Gephyrin homodimerization (Sola et al., 2004) and binding to the glycine receptor $\beta$ subunit (Meyer et al., 1995), and other proteins (Schema 6).

On the NL2 side, mapping of the Gephyrin binding site led to the identification of a 15-residue stretch within the NL2 cytoplasmic domain which is sufficient to confer Gephyrin-binding properties onto unrelated transmembrane proteins. This was exemplified by the fact that recombinant HA-tagged human CD8a containing this motif in its cytoplasmic domain acquired the capacity to form a complex with Gephyrin in heterologous cells (Figure $13 \mathrm{D}$ ). This motif is highly conserved in all Neuroligins, except for a putative phosphorylation site appearing at position 10 in Neuro- 
ligins 1,3 and 4 as noted above ( $\S 3.1 .5$ ), providing a structural basis to explain the experimental evidence showing that all Neuroligins can interact with Gephyrin.

In addition to identifying the sequence stretch sufficient for Gephyrin binding, a tyrosine residue within this stretch was shown to be necessary for Gephyrin binding in yeast. Mutation of Y770 in NL2 and Y782 in NL1 to alanine abolished Gephyrin interaction in yeast (Figure $11 \mathrm{~B}$ ). Crosslinking assays biochemically confirmed the importance of this residue for NL2-Gephyrin complex formation (Figure $13 \mathrm{~B}$ ). This tyrosine residue is part of a putative PPxY consensus sequence for WW domain binding, though the integrity of the PPxY motif was shown to be dispensable for Gephyrin binding as it remained unaffected by a P768A mutation in NL2 (Figure 12). The single point mutation $Y 770 A$ abolishing Gephyrin binding proved to be a useful tool throughout this study as examination of the differential effects of NL2 compared to NL2/Y770A in cell biological assays can be taken as indication that these effects are mechanistically mediated by the interaction of NL2 with Gephyrin. As this tyrosine residue is part of the 15-residue stretch shown to confer Gephyrin binding properties, this sequence can be considered as being necessary as well as sufficient for Gephyrin binding. Together with the fact that it is conserved in all Neuroligin homologues, this 15-residue sequence has the necessary properties to be considered a Gephyrin-binding motif characteristic to the Neuroligin family of proteins.

In the context of the neuron, NL2 and Gephyrin consistently co-localize at inhibitory postsynaptic sites (Varoqueaux et al., 2004). Additionally, exogenous surface clustering of neurons transfected with tagged NL2 was shown to co-cluster Gephyrin to the same sites (Graf et al., 2004). The data in these previous studies may represent the in vivo manifestation of the NL2-Gephyrin interaction. To address this possibility directly, the Y770A mutation abolishing Gephyrin-binding was examined as to its effects on the ability of NL2 to recruit Gephyrin to surface clusters in neurons. The assay described by Graf et al. (2004), where antibody-coated beads were used as a surface clustering agent, proved inappropriate to quantitatively compare the Gephyrin-recruitment capacity of different NL2 constructs. Beads induced tagged NL2 surface clusters only sporadically as many -indeed most- beads contacting transfected neurons did not appear to affect the distribution of exogenous NL2, making negative readouts uninformative and quantifica- 
tions liable to misinterpretation. This may be due to varying degrees of mechanical association between bead and plasma membrane, considering that the glycocalyx of the cell is expected to present a physical barrier that must be surmounted for the bead to have physical access to proteins on the plasma membrane.

A variation of this approach was developed that omitted the use of a bulky substrate for antibodies. Rather, neuronal cultures were treated with antibody in solution, washed and subsequently treated with anti-isotypic IgG polyclonal antibody. The second antibody, recognizing multiple epitopes of the first, is expected to create a molecular plexus of immunoglobulin molecules that will lead to formation of clusters of the first antibody and consequently of tagged NL2. This two-step antibody clustering approach has one principle difference from the methodology employing beads; namely that clusters are induced acutely and at room temperature to avoid their internalization. In contrast, experiments employing beads feature antibodies supported by a bulky substrate, allowing the beads to be applied to the culture at any stage and for however long. Nonetheless, in transfected rat hippocampal neuron cultures, two-step antibody clustering sequestered all of HA-NL2 surface immunoreactivity into clusters where endogenous Gephyrin would consistently co-cluster (Figure 17). The consistency of the readout and the large number of discrete clusters arising from this methodology made it suitable for quantifying the Gephyrin-recruitment capacity of different HA-tagged constructs.

Surprisingly, no mutation of the NL2 cytoplasmic domain appeared to affect its Gephyrin recruitment potential. In wild-type cells it thus appeared that recruitment of Gephyrin occurred independent of cytoplasmic domain sequences; in other words that clustering of the extracellular and transmembrane domains alone were sufficient to induce co-clustering. As Gephyrin is a cytosolic protein, this would imply that an endogenous neuronal component acts as a bridging molecule between the HA-tagged NL2 sequences outside the cytoplasmic domain to Gephyrin inside the cell. In view of the evidence herein that Neuroligin extracellular and transmembrane sequences mediate oligomerization of Neuroligins and that in hippocampal cultures NL2 mostly forms homodimers (Figure 25), a good candidate protein fitting the role of a bridging molecule as described above would be endogenous NL2. HA-tagged NL2 mutants would be expected to dimerize with endogenous NL2 lacking mutations. Such mixed dimers would present substrates for clustering 
due to the HA-tagged exogenous molecule. At the same time they would retain wild-type properties of the cytoplasmic domain from the endogenous molecule. As noted previously, this phenomenon would be expected to mask the phenotype of any loss-of-function mutations introduced and can potentially account for the lack of observable effects of Neuroligin cytoplasmic domain deletions reported in previous studies studies performed on wild-type backgrounds (Dresbach et al., 2004; Conroy et al., 2007).

To overcome the caveat of mutants pairing with endogenous NL2, the assay was repeated in a NL2-null background using neurons from NL2-deletion mutant mice. In this system, co-clustering of HA-NL2 with endogenous Gephyrin was much less robust. This is potentially due to the fact that neurons express NL2 for the first time only 24 hours prior to treatment. Before this, Gephyrin is expected to have a deficit in associating with the plasma membrane in the absence of NL2 (see below). Nonetheless, despite the reduced robustness of the readout and consequent low signal-to-noise ratio, significant and quantifiable differences were now apparent between different HA-tagged constructs.

Interference of the NL2-Gephyrin interaction via the Y770A mutation led to a reduction in the relative amount of co-clustered Gephyrin (Figure 18). This indicates that direct binding to Gephyrin contributes to the ability of NL2 surface clusters to recruit Gephyrin. Consistently, the CD8a chimaera containing the Gephyrinbinding motif (HA-CD8a/GB) acquired the ability to recruit Gephyrin above background levels as represented by the level of Gephyrin co-clustering with HA-CD8a. This is evidence that interaction with Gephyrin via the Gephyrin-binding motif clustered on the plasma membrane is sufficient to induce Gephyrin recruitment in neurons.

If one compares the level of Gephyrin recruitment between HA-NL2/Y770A and HA-CD8a, it becomes apparent that the mutated form of NL2 appears to partially retain its capacity to recruit Gephyrin even though it does not directly bind to it. Equivalently, comparison of the Gephyrin recruitment capacity of HA-NL2 and HACD8a/GB shows that in spite of being able to bind Gephyrin, the chimeric protein does not attain full capacity to recruit Gephyrin. Interpreted as such, it appears that 
direct Gephyrin binding is only one of the mechanisms in neurons that are involved in recruiting Gephyrin to surface clusters.

Following this rational, it becomes plausible to assume that NL2 and Gephyrin may additionally be linked via other components in the postsynaptic apparatus. One such possible candidate may be the Gephyrin binding protein Collybistin. Though there are no direct indications that it binds to NL2, there is functional evidence to suggest it (see below). Another potential candidate may be the AMPAtype glutamate receptor binding protein GRIP1. GRIP1 is one of the few PDZcontaining proteins to be present at both excitatory and inhibitory synapses (Charych et al., 2004). It was recently shown that GRIP1 isoforms can interact with Gephyrin (Yu et al., 2008). As the NL2 Y770A mutant was shown to retain a functional PDZ-binding domain (Figure $11 \mathrm{~B}$ ), it is conceivable that GRIP1 could mediate an additional indirect link between NL2 and Gephyrin. So far, though, there is no evidence for an NL2-GRIP1 interaction and GRIP1 PDZ domains 4 through 7 have been shown to selectively not interact with NL2 in yeast (Meyer et al., 2004).

Direct comparison of HA-tagged NL2 with CD8a constructs in the above assay is, however, liable to misinterpretation because of three bias factors. The expression levels of HA-tagged CD8a constructs were higher compared to the HA-tagged NL2 constructs. As Gephyrin recruitment capacity was expressed as the Gephyrin immunoreactivity versus the HA immunoreactivity per cluster, higher HA-tagged construct expression levels would be expected to under-estimate Gephyrin clustering ability. Following the same trend, the above analysis would tend to over-score an HA-tagged construct containing a Neuroligin extracellular domain over one with a CD8a extracellular domain. This bias stems from the fact that the analysis of extrasynaptic HA clusters may be contaminated with a small portion of synaptic clusters due to the fact that there is a minority of inhibitory contacts that lack Synapsin immunoreactivity (Bragina et al., 2007), which was the criterion used to distinguish synaptic from extrasynaptic clusters. An HA-tagged Neuroligin extracellular domain would be expected to be associated with synapses via presynaptic Neurexin binding while the CD8a sequence is not. Additionally, NL2 construct overexpression in neurons caused transfected neurons to receive higher innervation, consistent with previous observations (Levinson et al., 2005). This phenomenon would be expected to increase the proportion of synaptic clusters contaminating the 
analysis selectively for NL2 constructs. All the above possible errors tend in the direction of over-scoring NL2 constructs compared to CD8a constructs. It is thus possible that correction for these errors would show that HA-CD8a/GB has the same recruitment capacity as the positive control HA-NL2, and that HA-NL2/ Y770A has no significant recruitment capacity over the negative control HA-CD8a. As the extent to which these errors influence the readout was not determined, quantitative comparison of NL2 with CD8a constructs should be made with caution. However, the direct comparisons between mutant and wild-type versions of each protein is not affected by the above bias. Thus, these results clearly indicate that direct binding to Gephyrin is, at least in part, responsible for the ability of NL2 to recruit Gephyrin to plasma membrane clusters.

In neurons, the cellular manifestation of the NL2-Gephyrin interaction consists of the targeting of Gephyrin to plasma membrane sites of NL2 clusters. In heterologous expression systems like non-neuronal mammalian cell-lines, however, this does not appear to be the case. Gephyrin overexpressed in cell-lines forms characteristic cytoplasmic aggregates referred to as "blobs" (Kirsch et al., 1995) and does not associate with plasma membrane structures. Proteins known to interact with Gephyrin are often recruited to these aggregate structures (Meyer et al., 1995; Kins et al., 2000; Giesemann et al., 2003). NL2 however is only sporadically observed to associate with blobs (Figure 4). This was an indication that the interaction manifests itself only in a certain cellular environment; that it does not only require co-expression of the proteins, but is rather an inducible interaction that appears to be induced in neurons.

Complementing the NL2-Gephyrin mammalian cell-line expression system with a third co-expressing protein, namely Collybistin, drastically changed the distribution of both NL2 and Gephyrin and led to extenive co-clustering of all three coexpressed proteins at plasma membrane sites. Similar observations were made for NL1 and NL3 (Figure 15). Collybistin is a neuron-specific Gephyrin-binding protein homologous to GDP-GTP exchange factors (GEF) of the Dbl family which has been shown to be involved in Gephyrin targeting to the plasma membrane (Kins et al., 2000; Papadopoulos et al., 2007). Active forms of Collybistin, consisting of splice variant lacking a N-terminal SH3 domain (Harvey et al., 2004), readily redistribute Gephyrin, shifting its subcellular localization pattern from one characterized 
by cytoplasmic aggregates to one characterized by plasma membrane-associated microaggregates. Neuroligins, normally distributed diffusely throughout the plasma membrane, are enriched and often sequestered at the membrane sites of Gephyrin-Collybistin accumulation. Collybistin, thus appears to be a likely candidate for being the hypothetical factor that allows robust Neuroligin-Gephyrin coclustering in neurons and not in fibroblast cell-lines where Collybistin is normally not expressed.

At the plane of the membrane, Gephyrin and Neuroligins readily co-cluster, something not observed in cytoplasmic compartments. It is conceivable that Neuroligins, as transmembrane proteins, do not have access to cytoplasmic Gephyrin deposits because they are confined to compartments of the secretory or endocytotic pathways. Using soluble fusion proteins containing the NL2 cytoplasmic domain, however, did not lead to co-clustering with Gephyrin cytoplasmic aggregates. Additionally, Gephyrin and Neuroligins, regardless of the presence of Collybistin, do not prominently associate in intracellular membranous compartments. These observations indicate that permissiveness does not only involve overcoming segregation of the two proteins by membranous versus cytoplasmic compartmentalizations. Rather, the specific properties of the plasma membrane provide the proper environment for prominent co-clustering.

Collybistin has been shown to selectively activate the small G-protein Cdc42 (Reid et al., 1999; Xiang et al., 2006). Due to this potential signaling role of Collybistin, it is possible that the activated signaling cascade may be involved in inducing NL2Gephyrin co-clustering. To examine this, dominant negative mutants of Cdc42 were included in cell-lines co-expressing NL2, Gephyrin and Collybistin. Despite the expression of the dominant negative Cdc42 mutation, NL2-Gephyrin association proceeded unhindered. Equivalently, a constitutively active mutant of Cdc42 was unable to replace Collybistin in order to obtain NL2-Gephyrin co-clusters (data not shown). These preliminary observations indicate that the putative GEF activity of Collybistin towards Cdc42 is not necessary for Collybistin-mediated induction of Neuroligin-Gephyrin co-clusters. The notion that the putative GEF activity is an independent function of Collybistin is also supported by the observation that Gephyrin binding and GEF activity are either-or functions of Collybistin, as interaction with Gephyrin inhibits GEF activity in vitro (Xiang et al., 2006). A signaling role, 
however is not ruled out as the electrophoretic profile of Gephyrin is slightly altered in the presence of Collybistin. A minor diffuse band of slightly higher molecular weight can be observed upon co-transfection with Collybistin (Figure $15 \mathrm{~B}$ ). This may be suggestive of the occurrence of post-translationally modified Gephyrin, such as phopho-Gephyrin (Langosch et al., 1992), potentially induced by Collybistin signaling.

Another possible mode of induction of Neuroligin-Gephyrin co-clustering by Collybistin may be through an adaptor function of the latter. Collybistin was observed to co-cluster with Neuroligin-Gephyrin membrane clusters and is a known binding partner of Gephyrin (Kins et al., 2000; Grosskreutz et al., 2001). If Collybistin were to additionally interact with Neuroligins, it could serve as a liaison molecule to stabilize a NL2-Gephyrin complex, resulting in the observed robust coclustering. However, evidence of an interaction between Neuroligins and $\mathrm{SH} 3$ domain-deficient Collybistin is lacking. Co expression of HA-NL2 with GFPCB2sн3- did not lead to co-clustering of the two proteins even when surface HANL2 was clustered using a two-step antibody clustering protocol, arguing against an adaptor role for Collybistin in the complex. While it is plausible that NL2 may interact with SH3-containing forms of Collybistin, as suggested by compatibility in their primary structures and functional evidence (see below), there is no evidence to support such an interaction in the isoform lacking the $\mathrm{SH} 3$ domain discussed here. As the Y770A mutation on NL2 diminishes co-clustering (Figure 16), it appears that the NL2-Gephyrin interaction has a central role in forming these clusters at the plane of the plasma membrane when Collybistin is also present.

A surprising observation was made when Collybistin isoforms that retained the $\mathrm{N}$ terminal SH3 domain were examined. These isoforms have been shown to be inactive in terms of their capacity to target Gephyrin to plasma membrane sites. When co-expressed with Gephyrin in cell-lines, both are sequestered into cytoplasmic aggregates (Kins et al., 2000; Harvey et al., 2004; Figure 19). Upon cotransfection of NL2 together with Gephyrin and SH3 domain-containing Collybistin, the three proteins were again observed to redistribute into plasma membrane microaggregates (Figure 19 B). As expression of HA-CD8a, myc-NL1 or HA-NL3 instead of HA-NL2 did not lead to membrane clusters in this assay (Figure 20), it appears that NL2 specifically activates the membrane targeting capacity of SH3 
domain-containing Collybistin to be targeted along with Gephyrin to the plasma membrane.

Collybistin variants containing $\mathrm{SH} 3$ domains represent the vast majority of Collybistin transcripts and the only detectable isoforms at the protein level in vivo (Kins et al., 2000; Harvey et al., 2004; Papadopoulos et al., 2007). The fact that the physiologically relevant isoforms were shown to be inactive, while the active isoforms were absent in vivo appeared paradoxical in the Collybistin literature. To reconcile these datasets, it was hypothesized that a neuron-specific factor must activate native SH3-containing Collybistins in vivo (Harvey et al., 2004). The evidence presented herein indicates that NL2 can fulfill the criteria of this hypothetical activating factor.

Native Collybistin, in the presence of NL2, functions to recruit Gephyrin to the membrane similarly to Collybistin variants lacking the $\mathrm{SH} 3$ domain in the absence of NL2. Based on this observation, it may be speculated that NL2 functions to alleviate inhibition by the $\mathrm{SH} 3$ domain on Collybistin. As SH3 domains are typical protein interaction modules, it is reasonable to assume that such interactions participate in the mechanisms of inhibition. It remains unknown whether SH3-mediated inhibition occurs via an unidentified binding partner to the $\mathrm{SH} 3$ domain or whether it is of an intramolecular nature. Studies on the closest homologue of Collybistin, Asef, indicate that the $\mathrm{SH} 3$ domain of Asef acts as an intramolecular inhibitory domain, folding onto the catalytic GEF domain of Asef (Murayama et al., 2007). Given the high primary and tertiary structural similarities between Asef and Collybistin (Harvey et al., 2004; Gotthardt et al., 2007), it is likely that the latter, too, is regulated via $\mathrm{SH} 3$ domain-mediated intramolecular inhibition.

Following classical models of activation, which also apply in the case of Asef (Mitin et al., 2007), interaction of a protein with a putative intramolecular inhibitory subunit of Collybistin could lead to the disengagement of the intramolecular interaction. A putative lipid-binding Pleckstrin Homology $(\mathrm{PH})$ domain on the $\mathrm{C}$ terminus of Collybistin has been shown to be critical for its ability to target Gephyrin to the membrane (Harvey et al., 2004). Potential folding of the SH3 domain onto the PH domain, as is the case for Asef (Mitin et al., 2007), may mediate inhibition through a closed-state conformation that hinders lipid-binding. As the NL2 
cytoplasmic domain contains two putative PxxP consensus sequences and one poly-proline motif -both considered typical SH3-binding motifs (reviewed by Macias et al., 2002)- it will be interesting to examine whether NL2 can interact with the Collybistin SH3 domain and induce Collybistin to an open conformational state where membrane targeting through lipid binding is disinhibited.

Activation of Collybistin by NL2 was shown to depend on residue Y770, as mutation to alanine caused NL2 to lose its activating properties on Collybistin. As this residue has been shown to be critical for Gephyrin binding, it may be proposed that part of the activation mechanism of Collybistin involves interaction of NL2 with Gephyrin. However, inclusion of the Gephyrin-binding motif described herein in an unrelated membrane protein was not sufficient to activate Gephyrin recruitment to the membrane. This suggests, that while Gephyrin interaction with NL2 is necessary to activate Collybistin, the mechanistic role of NL2 does not only involve tethering of the Gephyrin-Collybistin complex to the membrane. This observation additionally implies that other sequences in the NL2 cytoplasmic domain have specific roles in the activation of Collybistin, consistent with the hypothesis of NL2mediated Collybistin disinhibition. It will be critical to examine whether the other Neuroligin paralogues share this property of NL2.

The in vitro data suggesting a role for NL2 in the disinhibition of Collybistin is supported by phenotypic similarities in cultured neurons from NL2- and Collybistindeletion mutant mice. Cultured hippocampal neurons lacking NL2 exhibit alterations in Gephyrin distribution. The number of Gephyrin puncta, as defined by intensity thresholding, across from presynaptic terminals was drastically reduced in the somatic compartment while the occurrence of cytosoplasmic Gephyrin aggregates in the somata of neurons lacking NL2 increased (Figure 21). Similar alterations in Gephyrin distribution were observed in neurons lacking Collybistin (Papadopouos et al., 2007). These phenotypic similarities may be taken as genetic evidence for a functional link between NL2 and Collybistin.

The change in the number of synaptic puncta in the somatic region may reflect a general depression of punctum fluorescence intensity, causing some puncta to diminish to subthreshols levels. The overall distribution pattern of Gephyrin appears to be characterized by a shift from postsynaptic to cytoplasmic, localization indicat- 
ing a deficit in the mechanisms of postsynaptic membrane targeting of Gephyrin. The fact that postsynaptic Gephyrin in the dendritic compartment persisted even in the absence of NL2 may imply distinct targeting mechanisms at work for Gephyrin between these neuronal compartments. Indeed the involvement of a microtubule motor-based mechanism shown to target Gephyrin to dendritic postsynaptic sites (Maas et al., 2006) could represent a NL2-independent mechanism for postsynaptic Gephyrin accumulation.

NL2 function has additionally been implicated in $\mathrm{GABA}_{A}$ receptor clustering as shown by the ability of Neurexin to cluster $\mathrm{GABA}_{\mathrm{A}}$ receptor subunits (Graf et al., 2004; Kang et al., 2007). Additional evidence comes from heterologous coexpression of NL2 with $\mathrm{GABA}_{\mathrm{A}}$ receptor subunits where exogenous surface clus-

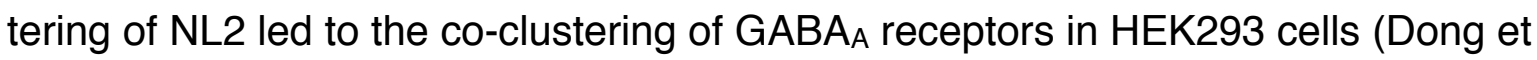
al, 2007). Evidence is presented herein showing that fibroblast cell-lines transfected with NL2, Gephyrin, Collybistin and $\alpha, \beta$ and $\gamma$ subunits of $\mathrm{GABA}_{A}$ receptors spontaneously form plasma membrane clusters where all these proteins cocluster, much like in their native organization at the postsynaptic membrane. This observation exemplifies that these protein constituents of inhibitory postsynapses are sufficient for self-organization, containing receptor, scaffold, adhesion and signaling components of the postsynaptic apparatus.

The interactions that lead to receptor clustering in the heterologous system where not dissected, and at least two modes of association can be inferred from the literature. As exogenous NL2 clustering leads to $\mathrm{GABA}_{\mathrm{A}}$ receptor co-clustering in HEK cells (Dong et al., 2007), it was suggested that a NL2-mediated link to receptor pentamers exists; either directly via in cis interactions at the level of the membrane, or indirectly through an endogenous component of HEK cells. A second possibility is a link of receptors via Gephyrin. Such a link has remained elusive for more than a decade since Gephyrin was shown to be present at GABAergic postsynaptic membranes (Craig et al., 1996). Recently a potential direct interaction between Gephyrin and the $\mathrm{GABA}_{\mathrm{A}}$ receptor a2 subunit was proposed (Tretter et al., 2008). Such a link may participate in the co-clustering observed herein as the a2 subunit was amongst the co-transfected constructs. In fact, this link can potentially account for the observations of Dong an colleagues (2007) who simillarly employed the $\mathrm{GABA}_{\mathrm{A}}$ receptor a2 subunit, keeping in mind that HEK cells are known 
to endogenously express Gephyrin (Fuhrmann et al., 2002), which is shown herein to bind Neuroligins.

Support of an in vivo role for NL2 in equipping the postsynaptic apparatus with $\mathrm{GABA}_{\mathrm{A}}$ receptors comes from observations on NL2 deletion-mutant mice. The overall levels of $\mathrm{GABA}_{A}$ receptor subunits examined in the brain of these mice remains unperturbed. However, synaptic preparations from brain homogenates revealed that the level of synaptic enrichment of the $\mathrm{GABA}_{A}$ receptor $\gamma 2$ subunit is $1 /$ 3 less than the wild-type levels (Figure 23). Other GABA $_{A}$ receptor subunits examined showed non-significant trends of reduction. However, the enrichment of these subunits in synaptic preparations was lower compared to that of the $\mathrm{Y} 2$. As $\mathrm{Y} 2$ is the $\mathrm{GABA}_{\mathrm{A}}$ receptor subunit with the most significant participation in phasic inhibition at postsynaptic sites (reviewed by Mody \& Pearce, 2004), reduction in this subunit in synaptic preparations may signify a general reduction in the synaptic pool of $\mathrm{GABA}_{\mathrm{A}}$ receptors. Gephyrin was also not observed to be decreased in synaptic preparations from NL2 deletion-mutant mice. However, in these preparations Gephyrin was not enriched (Figure 23). Evidence for the perturbation of Gephyrin synaptic targeting in NL2-deficient brains should be sought with a preparation where synaptic Gephyrin can be enriched over homogenate levels (Li et al., 2007). Whether the observed reduction in synaptic $\mathrm{GABA}_{\mathrm{A}}$ receptors is mediated directly by the loss of NL2 or indirectly via Gephyrin thus remains an open question.

Regardless of the specific mode of interaction of $\mathrm{GABA}_{\mathrm{A}}$ receptors with the NL2Gephyrin-Collybistin structures, the observation that receptors spontaneously accumulate at these structures serves as a proof-of-principle to indicate that NL2, Gephyrin and Collybistin are core components of the inhibitory postsynaptic scaffold, sufficient to define microdomains where receptors are to accumulate. Combining the new evidence as to the structural and functional relationships between the components of the inhibitory postsynapse presented herein along with previously documented data, a model of inhibitory postsynaptic assembly may be proposed (Schema 9). NL2 clustered at the surface of the plasma membrane can interact with cytoplasmic Gephyrin-Collybistin complexes via the Gephyrin-binding motif. This interaction is weak and serves to transiently recruit Gephyrin-Collybistin complexes to sites of NL2 clusters on the neuronal membrane. At these sites, 
A

Nucleation of the Gephyrin scaffold on the membrane by Neuroligin 2

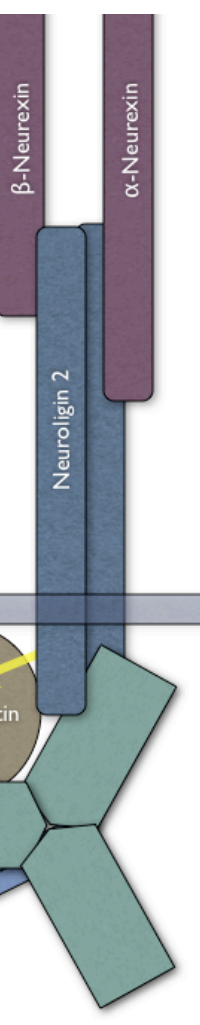

Microtubules

B

Expansion and membrane tethering of the Gephyrin scaffold

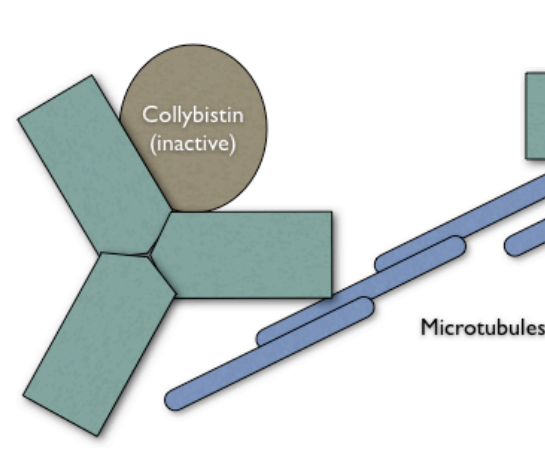

ephyrin

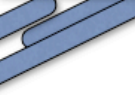

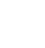

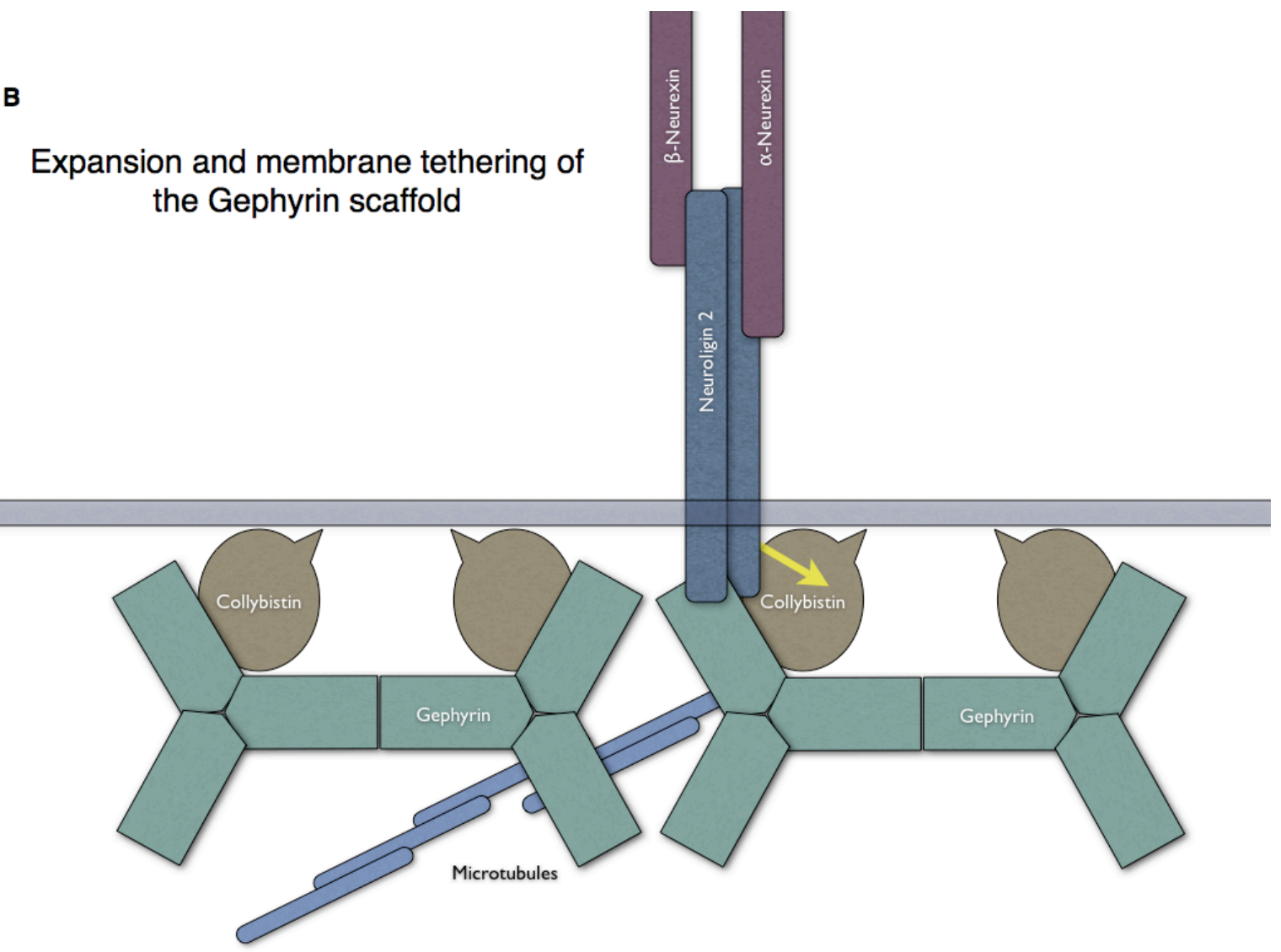




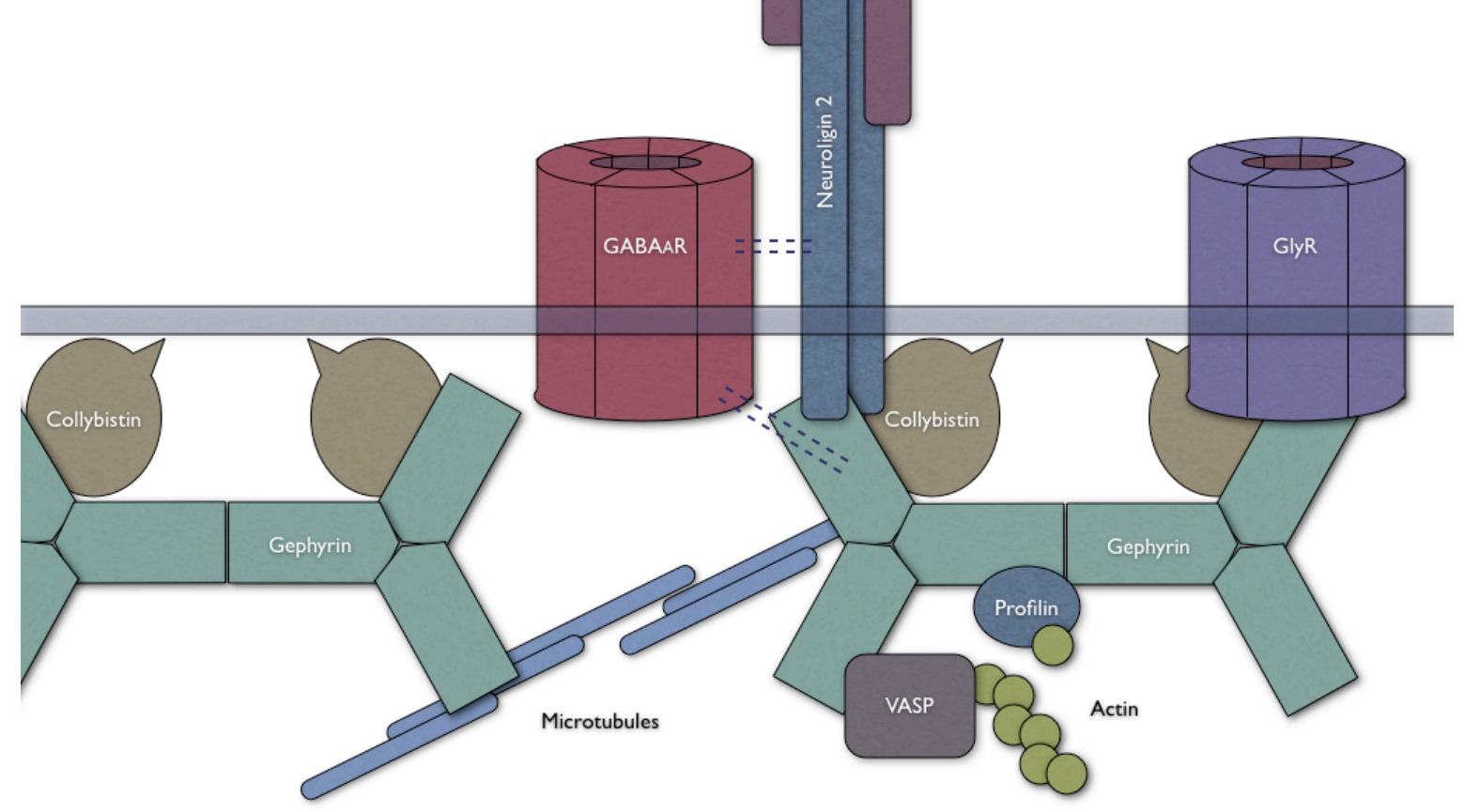

Schema 9. Assembly Model of the Inhibitory Postsynapse. (A) Neuroligin 2 dimers are clustered on the surface of the postsynaptic neuron by in trans interaction with a-Neurexins and $\beta$ Neurexins on contacting GABAergic or glycinergic axon terminals. Cytoplasmic GephyrinCollybistin complexes are transiently recruited to plasma membrane sites of Neuroligin 2 clusters via an interaction of the Neuroligin 2 cytoplasmic domain and the Gephyrin E-domain. Collybistin in complex with Gephyrin is activated at these nucleation sites by Neuroligin 2 and the Collybistin-Gephyrin complex is tethered to the plasma membrane via Collybistin. (B) Neuroligin 2 clusters further recruit and activate Gephyrin-Collybistin complexes, leading to the establishment of a membrane tethered postsynaptic Gephyrin scaffold. (C) Plasma membrane GlyRs and other Gephyrin-binding proteins are directly recruited to the scaffold. $\mathrm{GABA}_{A} R$ s are also recruited either directly or via an unidentified link to NL2-Gephyrin-Collybistin complexes. As a result, inhibitory receptors are clustered in precise apposition to presynaptic terminals releasing inhibitory transmitters.

Collybistin is regionally activated by NL2 to tether to the plasma membrane via $\mathrm{PH}$ domain-mediated binding to plasma membrane phosphoinositides. In such a model, the lipid tethering of the Gephyrin-Collybistin complex is the main structural link to the postsynaptic membrane as activated Collybistin is sufficient to link Gephyrin to the plasma membrane (Kins et al., 2000). The interaction of NL2 with Gephyrin, according to the model, would serve as a nucleation site for GephyrinCollybistin membrane microaggregate formation. Considering the NL2 link with presynaptic a-Neurexins, this nucleation site would serve to demarcate sites of in- 
hibitory terminal contact, signaling to the cytoplasmic side of the postsynaptic membrane the deployment site of the Gephyrin scaffold. Through direct and, potentially, indirect interactions, the scaffold serves to subsequently accumulate $\mathrm{GABA}_{\mathrm{A}}$ and glycine receptors in precise apposition to presynaptic terminals of GABA and/or glycine release.

\subsection{Principles of Neuroligin function and dysfunction}

A lot of attention has been focused on the potential role of Neuroligins during the early events of synaptogenesis. Overexpression and knockdown experiments have been interpreted to show that the principle function of Neuroligins lies within synapse formation, as they can quantitatively determine the innervation of transfected neurons in culture (Chih et al., 2005; Levinson et al., 2005;). Mouse genetic studies though have demonstrated that Neuroligins are dispensable for synapse formation and indeed have little effect on synapse numbers in vivo (Varoqueaux et al., 2006; Chubykin et al., 2007; Aramuni et al., submitted for publication). Though these two lines of evidence seem divergent, the in vitro data can be reinterpreted to avoid discrepancies.

Considering that synaptic contacts are initiated by transient adhesive interactions between two neurons, it is not surprising that the up- or down-regulation of a synaptic adhesion system in a neuron will, respectively, increase or decrease the stability of contacts resulting in greater numbers at a given time-point compared to un-manipulated neurons. It has been argued that generic strengthening of synaptic adhesion does not mediate the apparent changes in synapse numbers as the effect is specific to a set of adhesion proteins, including Neuroligins, and not to other synaptic adhesion molecules like Cadherins and N-CAMs (Table 1). This rational, however, does not take into account that bona fide synapses are designated by the accumulation of synaptic markers, or proteins indicative of synaptic differentiation. As Neuroligins and other proteins on Table 1 have been demonstrated to specifically induce synaptic differentiation at sites where they accumulate in vitro, it would be expected that a generic up-regulation of neuronal contacts upon overexpression of these proteins would additionally be accompanied by the recruitment 
of synaptic marker proteins, making these contacts register as synapses. Potential increases in neuronal contacts on cells transfected with adhesion proteins that do not induce synaptic differentiation would appear in the readouts of these studies as having no effect.

It thus appears that the most direct interpretation of the in vitro data identifies synaptic differentiation as being the specific effect mediated by Neuroligins over other synaptic adhesion molecules. This is in good accordance with in vivo data where adhesive competition is not manifested, probably due to the absence of mosaic neuronal populations, and faulty synaptic differentiation appears as the most prominent phenotype. It is additionally substantiated by the previously mentioned molecular mechanisms of association and/or recruitment identified herein for inhibitory synapses and previously for excitatory synapses. Together, these observations all point to a function of Neuroligins in initiating synaptic differentiation by recruiting postsynaptic components.

Having established a framework for Neuroligin function, evidence must be taken into account from studies conclusively revealing that that different Neuroligin paralogues have markedly different characteristics in postsynaptic differentiation. NL1 is mostly referred to in the context of glutamatergic synapses, while NL2 in the context of inhibitory synapses. So far, NL3 and NL4 are mostly associated with the study of Autism as their physiological roles are unclear. Yet, multiple Neuroligin deletion in mice and a series of in vitro studies indicate that the functions of Neuroligins are -or can be- partially overlapping.

The participation of NL1 in the proper assembly of the postsynaptic apparatus of glutamatergic terminals is exemplified by the study of NL1 deletion-mutant mice which exhibit diminished NMDA receptor-mediated synaptic responses (Chubykin et al., 2007). The most prominent structural determinants of NL1 function at the synapse are its extracellular interaction with presynaptic $\beta$-Neurexins (Ichtchenko et al., 1995) and its intracellular interaction with PDZ domain-containing proteins of the excitatory postsynaptic scaffold such as PSD-95 and S-SCAM (Irie et al., 1997; Hirao et al., 1998). NL2, equivalently, is necessary in vivo for proper postsynaptic responses to GABAergic and Glycinergic transmission (Aramuni et al., submitted for publication). It preferentially associates with splice site 4-containing 
$\beta$-Neurexins and a-Neurexins extracellularly (Boucard et al., 2005; Kang et al., 2007), while intracellularly it orchestrates the assembly of the postsynaptic scaffold through interaction with Gephyrin and activation of Collybistin.

It thus appears that Neuroligins, in spite of segregating to different synapse types, have a common regimen in their synaptic function; the deployment of postsynaptic scaffold in apposition to presynaptic release terminals. The parallels that can be drawn in the case of excitatory and inhibitory postsynapse assembly, however, have limits. PDZ domain-mediated interactions are thought to be involved in the multiple tethering of Neuroligins and other PSD components. In the case of NL2, the link to the scaffold seems to be structurally more complex. The postsynaptic cluster appears to be kept together by a combination of direct interaction with Gephyrin-Collybistin complexes and local activation of the association of the scaffolding complex with lipids on the postsynaptic membrane. Additionally, live imaging has identified pre-assembled particles of NL1 together with PSD-95 that move along the dendrite before associating with a presynaptic terminal (Gerrow et al., 2006). In the case of NL2, similar data-sets are not available and the order of events of postsynaptic assembly remains to be addressed. It does appear, however, that NL2 is one of the first postsynaptic proteins to form clusters even in the absence of Gephyrin (Varoqueaux et al., 2004).

Looking onto the other two members of the Neuroligin family, it appears that they too share the same basic properties as NL1 and NL2. Though much less is known about the functions of NL3 and NL4, it has been suggested that they associate primarily with excitatory synapses (Graf et al., 2006), while NL3 can associate with a subset of GABAergic synapses in culture. Nonetheless, NL3 and NL4 have the same basic properties as NL1 and NL2 in terms of binding to Neurexins, PDZcontaining proteins and Gephyrin. A crucial difference, however, appears to be the specificity of NL2 in the induction of Collybistin-mediated membrane tethering. It remains to be examined, whether NL4 may also have the capacity to activate Collybistin. As other Neuroligins can -on occasion- be found at inhibitory synapses, it may be that this special feature of NL2 has a critical function in the nucleation of the Gephyrin recruitment to plasma membrane sites contacted by presynaptic terminals. After the establishment of the inhibitory postsynaptic scaffold, the capacity of all Neuroligins to associate with Gephyrin means that other paralogues 
can associate with and may have auxiliary functions at certain inhibitory postsynapses. Other differences between Neuroligin paralogues have been suggested with regard to their binding affinities for Neurexins (Graf et al., 2006) and MAGuKs like PSD-95 (Irie et al., 1997), while Gephyrin binding may be differentially regulated by phosphorylation. It thus appears that all Neuroligins can associate with synaptic scaffolds, though different paralogues have distinct auxiliary features that may be significant in vivo where intricate balances exist between multiple interactions and functionalities.

Considering that Neuroligin paralogues can heterodimerize, an ensemble of Neuroligin dimer species with distinct properties is expected in vivo. Regardless of the paralogue combinations though, the core properties common to all Neuroligins ensure that any dimer can associate with any given synapse. At the same time, the diversity that arises from potential specific interaction partners to dimer combinations (Schema 5) may serve synapse-specific functions of different Neuroligin dimer species. The observation that the ratio of NL1 versus NL2 may be involved in determining the balance between excitation and inhibition (reviewed by Levinson \& El-Husseini, 2005) indicates the existence of an intricate balance between Neuroligin paralogues and the properties they confer upon a synapse to determine network function.

An extension of the notion that the balance of Neuroligin paralogues determines properties of neuron networks in the brain may be relevant in Neuroligin pathology. Mutations in NL3 and NL4 genes have been documented to co-segregate with pathological manifestations in cases of monogenic heritable autism (Jamain et al., 2003; Yan et al., 2004; Laumonnier et al., 2004). In the case of NL4, non sense mutations led to a truncated protein product of the extracellular domain. Recent evidence confirms that loss of NL4 is an aetiopathogenic factor as NL4 deletionmutant mice exhibit an autism-like behavioral phenotype (Jamain et al., 2008).

In the case of NL3, a single point mutation was implicated. This mutation has been suggested to cause faulty trafficking, leading to ER retention (Comoletti et al., 2005), while it was suggested to additionally interfere with oligomerization (De Jaco et al., 2006). It is shown herein that NL3 containing the autism-related mutation $\mathrm{R} 451 \mathrm{C}$ is retained in the ER by the same transmembrane mechanism that 
functions to prohibit Neuroligin monomers from being presented on the cell surface (Figure $26 \mathrm{C}$ ). A mutant mouse carrying the R451C mutation in NL3 exhibited an autism-like phenotype while the protein levels were reduced by $90 \%$ of physiological NL3 levels (Tabuchi et al., 2007). Despite the drastic reduction in protein levels, a distinct synaptic phenotype of the NL3/R451C mutant mouse was identified that was absent from the NL3 deletion-mutant mouse. This gain-of-function effect of the R451C mutation was observed as an increase in spontaneous inhibitory transmission in the somatosensory cortex.

At first glance, it seems puzzling that this point mutant which is rapidly degraded and does not properly traffic to reach the synapse can effect synaptic transmission. In light of the model of Neuroligin dimerization presented herein, NL3/R451C would be retained in the early secretory pathway via transmembrane-mediated binding to a resident protein. As this mechanism appears common to all Neuroligins, expression of mutant NL3 -which is the most abundantly expressed Neuroligin paralogue (Varoqueaux et al., 2006)- may overload the quality control mechanism, potentially affecting dimerization and trafficking of other Neuroligin paralogues. Surface expression of monomers or expression of aberrant dimers of NL1, NL2 or NL4 could potentially account for the alterations in synaptic transmission observed in the NL3/R451C mouse. Taken together these data show that perturbation in the levels of Neuroligin paralogues affects the properties of synaptic transmission.

Evidence is accumulating to suggest that multiple Neuroligins are not a product of system redundancy. Rather, they act in concert to determine network properties via their critical role in postsynapse differentiation. It appears that when paralogues are removed from the synaptic armament of Neuroligins, or their balances of expression are perturbed, synaptic transmission, and ultimately behavior, is altered. 


\section{Summary}

The establishment of accurate synaptic transmission underlies neural network processing and nervous system function. The organized deployment of specialized synaptic machinery is a key cellular process that shapes the synapse and its transmission properties, yet the molecular mechanisms involved in assembling the synaptic apparatus are largely unknown. This work has focused on the role of Neuroligins, a family of postsynaptic adhesion molecules, in mediating differentiation of inhibitory, GABAergic and glycinergic, postsynapses.

Evidence is provided indicating that the central role of Neuroligin 2 in the assembly of the inhibitory postsynapse is mediated through a molecular interaction with the inhibitory scaffolding protein Gephyrin, and a specific activation of the signaling protein Collybistin. Neuroligin 2 is shown to be critical for proper inhibitory postsynaptic scaffold recruitment in neurons and, together with Gephyrin and Collybistin, sufficient to mediate the recruitment of $\mathrm{GABA}_{\mathrm{A}}$ receptors.

A novel Gephyrin-binding motif characteristic of the Neuroligin protein family was identified. As all Neuroligins were shown to have the capacity to bind Gephyrin, it is possible that several Neuroligin paralogues have auxiliary functions at the inhibitory postsynapse. In accordance with this notion, the stoichiometry and composition of Neuroligin oligomers was determined in neurons to be dimeric and both homo- and heteromeric. Additionally, cellular mechanisms were identified which regulate the assembly and trafficking of Neuroligin oligomers, and evidence was provided indicating their potential involvement in Autism pathology. 


\section{References}

Alldred, M. J., Mulder-Rosi, J., Lingenfelter, S. E., Chen, G., and Lüscher, B. (2005). Distinct gamma2 subunit domains mediate clustering and synaptic function of postsynaptic GABAA receptors and gephyrin. J Neurosci 25, 594-603.

Alvarez, V. A., and Sabatini, B. L. (2007). Anatomical and physiological plasticity of dendritic spines. Annu Rev Neurosci 30, 79-97.

Anderson, T. R., Shah, P. A., and Benson, D. L. (2004). Maturation of glutamatergic and GABAergic synapse composition in hippocampal neurons. Neuropharmacology $47,694-705$.

Araç, D., Boucard, A. A., Ozkan, E., Strop, P., Newell, E., Südhof, T. C., and Brunger, A. T. (2007). Structures of Neuroligin-1 and the Neuroligin-1/Neurexin-1beta Complex Reveal Specific Protein-Protein and Protein-Ca(2+) Interactions. Neuron 56, 992-1003.

Aramuni G, Varoqueaux F, Poulopoulos A, Brose N, Zhang W. (2008). Neuroligin 2 Regulates the Maturation and Function of GABAergic and Glycinergic Synapses. Submitted for publication.

Bai, F., and Witzmann, F. A. (2007). Synaptosome proteomics. Subcell Biochem 43, 77-98.

Barnes, E. M. (2000). Intracellular trafficking of GABA(A) receptors. Life Sci 66, 1063-1070.

Bartos, M., Vida, I., and Jonas, P. (2007). Synaptic mechanisms of synchronized gamma oscillations in inhibitory interneuron networks. Nat Rev Neurosci 8, 45-56.

Bausen, M., Fuhrmann, J. C., Betz, H., and O'sullivan, G. A. (2005). The state of the actin cytoskeleton determines its association with gephyrin: role of ena/VASP family members. Mol Cell Neurosci 31, 376-386.

Betz, A., Okamoto, M., Benseler, F., and Brose, N. (1997). Direct interaction of the rat unc-13 homologue Munc13-1 with the $\mathrm{N}$ terminus of syntaxin. J Biol Chem 272, 2520-2526.

Biederer, T., Sara, Y., Mozhayeva, M., Atasoy, D., Liu, X., Kavalali, E. T., and Sudhof, T. C. (2002). SynCAM, a synaptic adhesion molecule that drives synapse assembly. Science 297, 1525-1531.

Bollag, G., and McCormick, F. (1991). Regulators and effectors of ras proteins. Annu Rev Cell Biol 7, 601-632.

Bolliger, M. F., Frei, K., Winterhalter, K. H., and Gloor, S. M. (2001). Identification of a novel neuroligin in humans which binds to PSD-95 and has a widespread expression. Biochem J 356, 581-588. 
Boucard, A. A., Chubykin, A. A., Comoletti, D., Taylor, P., and Südhof, T. C. (2005). A splice code for trans-synaptic cell adhesion mediated by binding of neuroligin 1 to alpha- and beta-neurexins. Neuron 48, 229-236.

Bragina, L., Candiracci, C., Barbaresi, P., Giovedì, S., Benfenati, F., and Conti, F. (2007). Heterogeneity of glutamatergic and GABAergic release machinery in cerebral cortex. Neuroscience 146, 1829-1840.

Brickley, S. G., Cull-Candy, S. G., and Farrant, M. (1999). Single-channel properties of synaptic and extrasynaptic GABAA receptors suggest differential targeting of receptor subtypes. J Neurosci 19, 2960-2973.

Brünig, I., Scotti, E., Sidler, C., and Fritschy, J. M. (2002a). Intact sorting, targeting, and clustering of gamma-aminobutyric acid $A$ receptor subtypes in hippocampal neurons in vitro. J Comp Neurol 443, 43-55.

Brünig, I., Suter, A., Knuesel, I., Lüscher, B., and Fritschy, J. M. (2002b). GABAergic terminals are required for postsynaptic clustering of dystrophin but not of $\mathrm{GABA}(\mathrm{A})$ receptors and gephyrin. J Neurosci 22, 4805-4813.

Budreck, E. C., and Scheiffele, P. (2007). Neuroligin-3 is a neuronal adhesion protein at GABAergic and glutamatergic synapses. Eur J Neurosci 26, 1738-1748.

Charych, E. I., Yu, W., Li, R., Serwanski, D. R., Miralles, C. P., Li, X., Yang, B. Y., Pinal, N., Walikonis, R., and De Blas, A. L. (2004). A four PDZ domain-containing splice variant form of GRIP1 is localized in GABAergic and glutamatergic synapses in the brain. J Biol Chem 279, 38978-38990.

Chen, X., Liu, H., Shim, A. H., Focia, P. J., and He, X. (2007). Structural basis for synaptic adhesion mediated by neuroligin-neurexin interactions. Nat Struct Mol Biol.

Chen, X., Vinade, L., Leapman, R. D., Petersen, J. D., Nakagawa, T., Phillips, T. M., Sheng, M., and Reese, T. S. (2005). Mass of the postsynaptic density and enumeration of three key molecules. Proc Natl Acad Sci USA 102, 11551-11556.

Chen, X., Winters, C., Azzam, R., Li, X., Galbraith, J. A., Leapman, R. D., and Reese, T. S. (2008). Organization of the core structure of the postsynaptic density. Proc Natl Acad Sci USA 105, 4453-4458.

Chih, B., Afridi, S. K., Clark, L., and Scheiffele, P. (2004). Disorder-associated mutations lead to functional inactivation of neuroligins. Hum Mol Genet 13, 14711477.

Chih, B., Engelman, H., and Scheiffele, P. (2005). Control of excitatory and inhibitory synapse formation by neuroligins. Science 307, 1324-1328.

Chih, B., Gollan, L., and Scheiffele, P. (2006). Alternative splicing controls selective trans-synaptic interactions of the neuroligin-neurexin complex. Neuron 51, 171-178. 
Choma, C., Gratkowski, H., Lear, J. D., and DeGrado, W. F. (2000). Asparaginemediated self-association of a model transmembrane helix. Nat Struct Biol 7, 161166.

Chubykin, A. A., Atasoy, D., Etherton, M. R., Brose, N., Kavalali, E. T., Gibson, J. R., and Südhof, T. C. (2007). Activity-Dependent Validation of Excitatory versus Inhibitory Synapses by Neuroligin-1 versus Neuroligin-2. Neuron 54, 919-931.

Chubykin, A. A., Liu, X., Comoletti, D., Tsigelny, I., Taylor, P., and Südhof, T. C. (2005). Dissection of synapse induction by neuroligins: effect of a neuroligin mutation associated with autism. J Biol Chem 280, 22365-22374.

Cline, H. (2005). Synaptogenesis: a balancing act between excitation and inhibition. Curr Biol 15, R203-205.

Colbran, R. J., and Brown, A. M. (2004). Calcium/calmodulin-dependent protein kinase II and synaptic plasticity. Curr Opin Neurobiol 14, 318-327.

Comoletti, D., De Jaco, A., Jennings, L. L., Flynn, R. E., Gaietta, G., Tsigelny, I., Ellisman, M. H., and Taylor, P. (2004). The Arg451Cys-neuroligin-3 mutation associated with autism reveals a defect in protein processing. J Neurosci 24,4889 4893.

Comoletti, D., Flynn, R., Jennings, L. L., Chubykin, A., Matsumura, T., Hasegawa, H., Südhof, T. C., and Taylor, P. (2003). Characterization of the interaction of a recombinant soluble neuroligin-1 with neurexin-1beta. J Biol Chem 278, 5049750505.

Comoletti, D., Flynn, R. E., Boucard, A. A., Demeler, B., Schirf, V., Shi, J., Jennings, L. L., Newlin, H. R., Südhof, T. C., and Taylor, P. (2006). Gene selection, alternative splicing, and post-translational processing regulate neuroligin selectivity for beta-neurexins. Biochemistry 45, 12816-12827.

Comoletti, D., Grishaev, A., Whitten, A. E., Tsigelny, I., Taylor, P., and Trewhella, J. (2007). Synaptic Arrangement of the Neuroligin/beta-Neurexin Complex Revealed by X-Ray and Neutron Scattering. Structure 15, 693-705.

Connors, B. W., and Long, M. A. (2004). Electrical synapses in the mammalian brain. Annu Rev Neurosci 27, 393-418.

Conroy, W. G., Nai, Q., Ross, B., Naughton, G., and Berg, D. K. (2007). Postsynaptic neuroligin enhances presynaptic inputs at neuronal nicotinic synapses. Dev Biol 307, 79-91.

Coyle, J. E., and Nikolov, D. B. (2004). GABARAP: lessons for synaptogenesis. The Neuroscientist: a review journal bringing neurobiology, neurology and psychiatry 9, 205-216.

Craig, A. M., Banker, G., Chang, W., McGrath, M. E., and Serpinskaya, A. S. (1996). Clustering of gephyrin at GABAergic but not glutamatergic synapses in cultured rat hippocampal neurons. J Neurosci 16, 3166-3177. 
Craig, A. M., and Kang, Y. (2007). Neurexin-neuroligin signaling in synapse development. Proc Natl Acad Sci U S A.

Craven, S. E., El-Husseini, A. E., and Bredt, D. S. (1999). Synaptic targeting of the postsynaptic density protein PSD-95 mediated by lipid and protein motifs. Neuron 22, 497-509.

Crivellato, E., and Ribatti, D. (2007). Soul, mind, brain: Greek philosophy and the birth of neuroscience. Brain Res Bull 71, 327-336.

Cull-Candy, S., Brickley, S., and Farrant, M. (2001). NMDA receptor subunits: diversity, development and disease. Curr Opin Neurobiol 11, 327-335.

Daniels, M. P. (1997). Intercellular communication that mediates formation of the neuromuscular junction. Mol Neurobiol 14, 143-170.

De Jaco, A., Comoletti, D., Kovarik, Z., Gaietta, G., Radic, Z., Lockridge, O., Ellisman, M. H., and Taylor, P. (2006). A mutation linked with autism reveals a common mechanism of endoplasmic reticulum retention for the alpha,beta-hydrolase fold protein family. J Biol Chem 281, 9667-9676.

Dean, C., and Dresbach, T. (2005). Neuroligins and neurexins: linking cell adhesion, synapse formation and cognitive function. Trends Neurosci 29, 21-29.

Dean, C., Scholl, F. G., Choih, J., DeMaria, S., Berger, J., Isacoff, E., and Scheiffele, P. (2003). Neurexin mediates the assembly of presynaptic terminals. Nat Neurosci 6, 708-716.

DeFeudis, F. V. (1983). Psychoactive agents and GABA-receptors. Pharmacological research communications 15, 29-39.

Deng, F., Price, M. G., Davis, C. F., Mori, M., and Burgess, D. L. (2006). Stargazin and other transmembrane AMPA receptor regulating proteins interact with synaptic scaffolding protein MAGI-2 in brain. J Neurosci 26, 7875-7884.

Dickson, B. J. (2002). Molecular mechanisms of axon guidance. Science 298, 1959-1964.

Dong, N., Qi, J., and Chen, G. (2007). Molecular reconstitution of functional GABAergic synapses with expression of neuroligin-2 and $\mathrm{GABA}(\mathrm{A})$ receptors. Mol Cell Neurosci.

Dresbach, T., Neeb, A., Meyer, G., Gundelfinger, E. D., and Brose, N. (2004). Synaptic targeting of neuroligin is independent of neurexin and SAP90/PSD95 binding. Mol Cell Neurosci 27, 227-235.

Dumoulin, A., Triller, A., and Dieudonné, S. (2001). IPSC kinetics at identified GABAergic and mixed GABAergic and glycinergic synapses onto cerebellar Golgi cells. J Neurosci 21, 6045-6057. 
Elias, G. M., Funke, L., Stein, V., Grant, S. G., Bredt, D. S., and Nicoll, R. A. (2006). Synapse-specific and developmentally regulated targeting of AMPA receptors by a family of MAGUK scaffolding proteins. Neuron 52, 307-320.

Essrich, C., Lorez, M., Benson, J. A., Fritschy, J. M., and Lüscher, B. (1999). Postsynaptic clustering of major GABAA receptor subtypes requires the gamma 2 subunit and gephyrin. Nat Neurosci 1, 563-571.

Fabrichny, I. P., Leone, P., Sulzenbacher, G., Comoletti, D., Miller, M. T., Taylor, P., Bourne, Y., and Marchot, P. (2007). Structural Analysis of the Synaptic Protein Neuroligin and Its beta-Neurexin Complex: Determinants for Folding and Cell Adhesion. Neuron 56, 979-991.

Feng, G., Tintrup, H., Kirsch, J., Nichol, M. C., Kuhse, J., Betz, H., and Sanes, J. R. (1998). Dual requirement for gephyrin in glycine receptor clustering and molybdoenzyme activity. Science 282, 1321-1324.

Fleck, M. W. (2006). Glutamate receptors and endoplasmic reticulum quality control: looking beneath the surface. The Neuroscientist: a review journal bringing neurobiology, neurology and psychiatry 12, 232-244.

Fox, M. A., and Umemori, H. (2006). Seeking long-term relationship: axon and target communicate to organize synaptic differentiation. J Neurochem 97, 1215-1231.

Fritschy, J. M., Johnson, D. K., Möhler, H., and Rudolph, U. (1998a). Independent assembly and subcellular targeting of GABA(A)-receptor subtypes demonstrated in mouse hippocampal and olfactory neurons in vivo. Neurosci Lett 249, 99-102.

Fritschy, J. M., and Möhler, H. (1995). GABAA-receptor heterogeneity in the adult rat brain: differential regional and cellular distribution of seven major subunits. $J$ Comp Neurol 359, 154-194.

Fritschy, J. M., Weinmann, O., Wenzel, A., and Benke, D. (1998b). Synapsespecific localization of NMDA and GABA(A) receptor subunits revealed by antigenretrieval immunohistochemistry. J Comp Neurol 390, 194-210.

Fuhrmann, J. C., Kins, S., Rostaing, P., El Far, O., Kirsch, J., Sheng, M., Triller, A., Betz, H., and Kneussel, M. (2002). Gephyrin interacts with Dynein light chains 1 and 2, components of motor protein complexes. J Neurosci 22, 5393-5402.

Fujita, M., Sato, K., Sato, M., Inoue, T., Kozuka, T., and Tohyama, M. (1991). Regional distribution of the cells expressing glycine receptor beta subunit mRNA in the rat brain. Brain Res 560, 23-37.

Füllekrug, J., Boehm, J., Röttger, S., Nilsson, T., Mieskes, G., and Schmitt, H. D. (1997). Human Rer1 is localized to the Golgi apparatus and complements the deletion of the homologous Rer1 protein of Saccharomyces cerevisiae. Eur J Cell Biol 74, 31-40.

Gautam, M., Noakes, P. G., Moscoso, L., Rupp, F., Scheller, R. H., Merlie, J. P., and Sanes, J. R. (1996). Defective neuromuscular synaptogenesis in agrindeficient mutant mice. Cell $85,525-535$. 
Gautam, M., Noakes, P. G., Mudd, J., Nichol, M., Chu, G. C., Sanes, J. R., and Merlie, J. P. (1995). Failure of postsynaptic specialization to develop at neuromuscular junctions of rapsyn-deficient mice. Nature 377, 232-236.

Gay, E. A., and Yakel, J. L. (2007). Gating of nicotinic ACh receptors; new insights into structural transitions triggered by agonist binding that induce channel opening. J Physiol (Lond) 584, 727-733.

Gerrow, K., Romorini, S., Nabi, S. M., Colicos, M. A., Sala, C., and El-Husseini, A. (2006). A preformed complex of postsynaptic proteins is involved in excitatory synapse development. Neuron 49, 547-562.

Giesemann, T., Schwarz, G., Nawrotzki, R., Berhörster, K., Rothkegel, M., Schlüter, K., Schrader, N., Schindelin, H., Mendel, R. R., Kirsch, J., and Jockusch, B. M. (2003). Complex formation between the postsynaptic scaffolding protein gephyrin, profilin, and Mena: a possible link to the microfilament system. J Neurosci $23,8330-8339$.

Glass, D. J., Bowen, D. C., Stitt, T. N., Radziejewski, C., Bruno, J., Ryan, T. E., Gies, D. R., Shah, S., Mattsson, K., Burden, S. J., et al. (1996). Agrin acts via a MuSK receptor complex. Cell 85, 513-523.

Gotthardt, K., and Ahmadian, M. R. (2007). Asef is a Cdc42-specific guanine nucleotide exchange factor. Biol Chem 388, 67-71.

Grady, R. M., Wozniak, D. F., Ohlemiller, K. K., and Sanes, J. R. (2006). Cerebellar synaptic defects and abnormal motor behavior in mice lacking alpha- and betadystrobrevin. J Neurosci 26, 2841-2851.

Graf, E. R., Kang, Y., Hauner, A. M., and Craig, A. M. (2006). Structure function and splice site analysis of the synaptogenic activity of the neurexin-1 beta LNS domain. J Neurosci 26, 4256-4265.

Graf, E. R., Zhang, X., Jin, S. X., Linhoff, M. W., and Craig, A. M. (2004). Neurexins induce differentiation of GABA and glutamate postsynaptic specializations via neuroligins. Cell 119, 1013-1026.

Greger, I. H., Ziff, E. B., and Penn, A. C. (2007). Molecular determinants of AMPA receptor subunit assembly. Trends Neurosci 30, 407-416.

Grosskreutz, Y., Hermann, A., Kins, S., Fuhrmann, J. C., Betz, H., and Kneussel, M. (2001). Identification of a gephyrin-binding motif in the GDP/GTP exchange factor collybistin. Biol Chem 382, 1455-1462.

Harms, K. J., and Craig, A. M. (2005). Synapse composition and organization following chronic activity blockade in cultured hippocampal neurons. J Comp Neurol 490, $72-84$.

Harvey, K., Duguid, I. C., Alldred, M. J., Beatty, S. E., Ward, H., Keep, N. H., Lingenfelter, S. E., Pearce, B. R., Lundgren, J., Owen, M. J., et al. (2004). The GDPGTP exchange factor collybistin: an essential determinant of neuronal gephyrin clustering. J Neurosci 24, 5816-5826. 
Hata, Y., Butz, S., and Südhof, T. C. (1996). CASK: a novel dlg/PSD95 homolog with an N-terminal calmodulin-dependent protein kinase domain identified by interaction with neurexins. J Neurosci 16, 2488-2494.

Hata, Y., Davletov, B., Petrenko, A. G., Jahn, R., and Südhof, T. C. (1993). Interaction of synaptotagmin with the cytoplasmic domains of neurexins. Neuron 10, 307-315.

Hirao, K., Hata, Y., Ide, N., Takeuchi, M., Irie, M., Yao, I., Deguchi, M., Toyoda, A., Sudhof, T. C., and Takai, Y. (1998). A novel multiple PDZ domain-containing molecule interacting with $\mathrm{N}$-methyl-D-aspartate receptors and neuronal cell adhesion proteins. J Biol Chem 273, 21105-21110.

Hoffman, R. C., Jennings, L. L., Tsigelny, I., Comoletti, D., Flynn, R. E., Sudhof, T. C., and Taylor, P. (2004). Structural characterization of recombinant soluble rat neuroligin 1: mapping of secondary structure and glycosylation by mass spectrometry. Biochemistry 43, 1496-1506.

Holt, M. R., and Koffer, A. (2001). Cell motility: proline-rich proteins promote protrusions. Trends Cell Biol 11, 38-46.

Husi, H., Ward, M. A., Choudhary, J. S., Blackstock, W. P., and Grant, S. G. (2000). Proteomic analysis of NMDA receptor-adhesion protein signaling complexes. Nat Neurosci 3, 661-669.

Ichtchenko, K., Hata, Y., Nguyen, T., Ullrich, B., Missler, M., Moomaw, C., and Südhof, T. C. (1995). Neuroligin 1: a splice site-specific ligand for beta-neurexins. Cell 81, 435-443.

Ichtchenko, K., Nguyen, T., and Südhof, T. C. (1996). Structures, alternative splicing, and neurexin binding of multiple neuroligins. J Biol Chem 271, 2676-2682.

lida, J., Hirabayashi, S., Sato, Y., and Hata, Y. (2004). Synaptic scaffolding molecule is involved in the synaptic clustering of neuroligin. Mol Cell Neurosci 27, 497508.

Irie, M., Hata, Y., Takeuchi, M., Ichtchenko, K., Toyoda, A., Hirao, K., Takai, Y., Rosahl, T. W., and Südhof, T. C. (1997). Binding of neuroligins to PSD-95. Science 277, 1511-1515.

Jamain, S., Quach, H., Betancur, C., Råstam, M., Colineaux, C., Gillberg, I. C., Soderstrom, H., Giros, B., Leboyer, M., Gillberg, C., et al. (2003). Mutations of the X-linked genes encoding neuroligins NLGN3 and NLGN4 are associated with autism. Nat Genet 34, 27-29.

Jamain, S., Radyushkin, K., Hammerschmidt, K., Granon, S., Boretius, S., Varoqueaux, F., Ramanantsoa, N., Gallego, J., Ronnenberg, A., Winter, D., et al. (2008). Reduced social interaction and ultrasonic communication in a mouse model of monogenic heritable autism. Proc Natl Acad Sci USA 105, 1710-1715.

Jonas, P., Bischofberger, J., and Sandkühler, J. (1998). Corelease of two fast neurotransmitters at a central synapse. Science 281, 419-424. 
Jones, D. H., and Matus, A. I. (1974). Isolation of synaptic plasma membrane from brain by combined flotation-sedimentation density gradient centrifugation. Biochim Biophys Acta 356, 276-287.

Kaether, C., Haass, C., and Steiner, H. (2006). Assembly, trafficking and function of gamma-secretase. Neuro-degenerative diseases 3, 275-283.

Kaether, C., Scheuermann, J., Fassler, M., Zilow, S., Shirotani, K., Valkova, C., Novak, B., Kacmar, S., Steiner, H., and Haass, C. (2007). Endoplasmic reticulum retention of the gamma-secretase complex component Pen2 by Rer1. EMBO Rep 8, 743-748.

Kang, Y., Zhang, X., Dobie, F., Wu, H., and Craig, A. M. (2007). Induction of gabaergic postsynaptic differentiation by alpha -neurexins. J Biol Chem.

Kato, Y., Nagata, K., Takahashi, M., Lian, L., Herrero, J. J., Sudol, M., and Tanokura, M. (2004). Common mechanism of ligand recognition by group II/III WW domains: redefining their functional classification. J Biol Chem 279, 31833-31841.

Kay, B. K., Williamson, M. P., and Sudol, M. (2000). The importance of being proline: the interaction of proline-rich motifs in signaling proteins with their cognate domains. FASEB J 14, 231-241.

Kennedy, M. B. (1993). The postsynaptic density. Curr Opin Neurobiol 3, 732-737.

Kim, S., Burette, A., Chung, H. S., Kwon, S. K., Woo, J., Lee, H. W., Kim, K., Kim, H., Weinberg, R. J., and Kim, E. (2006). NGL family PSD-95-interacting adhesion molecules regulate excitatory synapse formation. Nat Neurosci 9, 1294-1301.

Kins, S., Betz, H., and Kirsch, J. (2000). Collybistin, a newly identified brainspecific GEF, induces submembrane clustering of gephyrin. Nat Neurosci 3, 22-29.

Kirsch, J., Kuhse, J., and Betz, H. (1995). Targeting of glycine receptor subunits to gephyrin-rich domains in transfected human embryonic kidney cells. Mol Cell Neurosci $6,450-461$.

Kirsch, J., Langosch, D., Prior, P., Littauer, U. Z., Schmitt, B., and Betz, H. (1991). The 93-kDa glycine receptor-associated protein binds to tubulin. J Biol Chem 266, 22242-22245.

Kirsch, J., Wolters, I., Triller, A., and Betz, H. (1993). Gephyrin antisense oligonucleotides prevent glycine receptor clustering in spinal neurons. Nature 366, 745748.

Kittler, J. T., McAinsh, K., and Moss, S. J. (2002). Mechanisms of GABAA receptor assembly and trafficking: implications for the modulation of inhibitory neurotransmission. Mol Neurobiol 26, 251-268.

Kneussel, M., and Betz, H. (2000). Clustering of inhibitory neurotransmitter receptors at developing postsynaptic sites: the membrane activation model. Trends Neurosci 23, 429-435. 
Kneussel, M., Haverkamp, S., Fuhrmann, J. C., Wang, H., Wässle, H., Olsen, R. W., and Betz, H. (2000). The gamma-aminobutyric acid type A receptor (GABAAR)-associated protein GABARAP interacts with gephyrin but is not involved in receptor anchoring at the synapse. Proc Natl Acad Sci USA 97, 85948599.

Knuesel, I., Mastrocola, M., Zuellig, R. A., Bornhauser, B. C., Schaub, M. C., and Fritschy, J. M. (1999). Short communication: altered synaptic clustering of GABAA receptors in mice lacking dystrophin (mdx mice). Eur J Neurosci 11, 4457-4462.

Ko, J., Kim, S., Chung, H. S., Kim, K., Han, K., Kim, H., Jun, H., Kaang, B. K., and Kim, E. (2006). SALM synaptic cell adhesion-like molecules regulate the differentiation of excitatory synapses. Neuron 50, 233-245.

Koehnke, J., Jin, X., Budreck, E. C., Posy, S., Scheiffele, P., Honig, B., and Shapiro, L. (2008). Crystal structure of the extracellular cholinesterase-like domain from neuroligin-2. Proc Natl Acad Sci USA.

Kralic, J. E., Sidler, C., Parpan, F., Homanics, G. E., Morrow, A. L., and Fritschy, J. M. (2006). Compensatory alteration of inhibitory synaptic circuits in cerebellum and thalamus of gamma-aminobutyric acid type A receptor alpha1 subunit knockout mice. J Comp Neurol 495, 408-421.

Krause, M., Dent, E. W., Bear, J. E., Loureiro, J. J., and Gertler, F. B. (2003). Ena/ VASP proteins: regulators of the actin cytoskeleton and cell migration. Annu Rev Cell Dev Biol 19, 541-564.

Krnjević, K., and Schwartz, S. (1966). Is gamma-aminobutyric acid an inhibitory transmitter? Nature 211, 1372-1374.

Kurschner, C., Mermelstein, P. G., Holden, W. T., and Surmeier, D. J. (1998). CIPP, a novel multivalent PDZ domain protein, selectively interacts with Kir4.0 family members, NMDA receptor subunits, neurexins, and neuroligins. Mol Cell Neurosci $11,161-172$.

Lajoix, A. D., Gross, R., Aknin, C., Dietz, S., Granier, C., and Laune, D. (2004). Cellulose membrane supported peptide arrays for deciphering protein-protein interaction sites: the case of PIN, a protein with multiple natural partners. Mol Divers 8, 281-290.

Langosch, D., Hoch, W., and Betz, H. (1992). The 93 kDa protein gephyrin and tubulin associated with the inhibitory glycine receptor are phosphorylated by an endogenous protein kinase. FEBS Lett 298, 113-117.

Laumonnier, F., Bonnet-Brilhault, F., Gomot, M., Blanc, R., David, A., Moizard, M. P., Raynaud, M., Ronce, N., Lemonnier, E., Calvas, P., et al. (2004). X-linked mental retardation and autism are associated with a mutation in the NLGN4 gene, a member of the neuroligin family. Am J Hum Genet 74, 552-557.

Lee Minsky, M. a. r. v. i. n. (1986). The Society of Mind. 336. 
Leonard, A. S., Davare, M. A., Horne, M. C., Garner, C. C., and Hell, J. W. (1998). SAP97 is associated with the alpha-amino-3-hydroxy-5-methylisoxazole-4propionic acid receptor GluR1 subunit. J Biol Chem 273, 19518-19524.

Levinson, J. N., Chéry, N., Huang, K., Wong, T. P., Gerrow, K., Kang, R., Prange, O., Wang, Y. T., and El-Husseini, A. (2005). Neuroligins mediate excitatory and inhibitory synapse formation: involvement of PSD-95 and neurexin-1beta in neuroligin-induced synaptic specificity. J Biol Chem 280, 17312-17319.

Levinson, J. N., and El-Husseini, A. (2005). Building excitatory and inhibitory synapses: balancing neuroligin partnerships. Neuron 48, 171-174.

Li, R. W., Yu, W., Christie, S., Miralles, C. P., Bai, J., Loturco, J. J., and De Blas, A. L. (2005). Disruption of postsynaptic GABA receptor clusters leads to decreased GABAergic innervation of pyramidal neurons. J Neurochem 95, 756-770.

Li, X., Serwanski, D. R., Miralles, C. P., Bahr, B. A., and De Blas, A. L. (2007). Two pools of Triton X-100-insoluble $\mathrm{GABA}(\mathrm{A})$ receptors are present in the brain, one associated to lipid rafts and another one to the post-synaptic GABAergic complex. J Neurochem 102, 1329-1345.

Lynch, J. W. (2004). Molecular structure and function of the glycine receptor chloride channel. Physiol Rev 84, 1051-1095.

Lévi, S., Grady, R. M., Henry, M. D., Campbell, K. P., Sanes, J. R., and Craig, A. M. (2002). Dystroglycan is selectively associated with inhibitory GABAergic synapses but is dispensable for their differentiation. J Neurosci 22, 4274-4285.

Lévi, S., Logan, S. M., Tovar, K. R., and Craig, A. M. (2004). Gephyrin is critical for glycine receptor clustering but not for the formation of functional GABAergic synapses in hippocampal neurons. J Neurosci 24, 207-217.

Lüddens, H., and Wisden, W. (1991). Function and pharmacology of multiple GABAA receptor subunits. Trends Pharmacol Sci 12, 49-51.

Lüscher, B., and Keller, C. A. (2004). Regulation of GABAA receptor trafficking, channel activity, and functional plasticity of inhibitory synapses. Pharmacol Ther 102, 195-221.

Ma, D., and Jan, L. Y. (2002). ER transport signals and trafficking of potassium channels and receptors. Curr Opin Neurobiol 12, 287-292.

Ma, X. M., Huang, J., Wang, Y., Eipper, B. A., and Mains, R. E. (2003). Kalirin, a multifunctional Rho guanine nucleotide exchange factor, is necessary for maintenance of hippocampal pyramidal neuron dendrites and dendritic spines. J Neurosci 23, 10593-10603.

Maas, C., Tagnaouti, N., Loebrich, S., Behrend, B., Lappe-Siefke, C., and Kneussel, M. (2006). Neuronal cotransport of glycine receptor and the scaffold protein gephyrin. J Cell Biol 172, 441-451. 
Macias, M. J., Wiesner, S., and Sudol, M. (2002). WW and SH3 domains, two different scaffolds to recognize proline-rich ligands. FEBS Lett 513, 30-37.

Mammoto, A., Sasaki, T., Asakura, T., Hotta, I., Imamura, H., Takahashi, K., Matsuura, Y., Shirao, T., and Takai, Y. (1998). Interactions of drebrin and gephyrin with profilin. Biochem Biophys Res Commun 243, 86-89.

Meier, J., Vannier, C., Sergé, A., Triller, A., and Choquet, D. (2001). Fast and reversible trapping of surface glycine receptors by gephyrin. Nat Neurosci $4,253-$ 260.

Meyer, G., Kirsch, J., Betz, H., and Langosch, D. (1995). Identification of a gephyrin binding motif on the glycine receptor beta subunit. Neuron 15, 563-572.

Meyer, G., Varoqueaux, F., Neeb, A., Oschlies, M., and Brose, N. (2004). The complexity of PDZ domain-mediated interactions at glutamatergic synapses: a case study on neuroligin. Neuropharmacology 47, 724-733.

Migaud, M., Charlesworth, P., Dempster, M., Webster, L. C., Watabe, A. M., Makhinson, M., He, Y., Ramsay, M. F., Morris, R. G., Morrison, J. H., et al. (1998). Enhanced long-term potentiation and impaired learning in mice with mutant postsynaptic density-95 protein. Nature 396, 433-439.

Misgeld, T., Kummer, T. T., Lichtman, J. W., and Sanes, J. R. (2005). Agrin promotes synaptic differentiation by counteracting an inhibitory effect of neurotransmitter. Proc Natl Acad Sci USA 102, 11088-11093.

Missler, M., Fernandez-Chacon, R., and Südhof, T. C. (1998). The making of neurexins. J Neurochem 71, 1339-1347.

Missler, M., Zhang, W., Rohlmann, A., Kattenstroth, G., Hammer, R. E., Gottmann, K., and Südhof, T. C. (2003). Alpha-neurexins couple Ca2+ channels to synaptic vesicle exocytosis. Nature 423, 939-948.

Mitin, N., Betts, L., Yohe, M. E., Der, C. J., Sondek, J., and Rossman, K. L. (2007). Release of autoinhibition of ASEF by APC leads to CDC42 activation and tumor suppression. Nat Struct Mol Biol 14, 814-823.

Mody, I., and Pearce, R. A. (2004). Diversity of inhibitory neurotransmission through $\operatorname{GABA}(\mathrm{A})$ receptors. Trends Neurosci 27, 569-575.

Moss, S. J., and Smart, T. G. (2001). Constructing inhibitory synapses. Nat Rev Neurosci 2, 240-250.

Murayama, K., Shirouzu, M., Kawasaki, Y., Kato-Murayama, M., HanawaSuetsugu, K., Sakamoto, A., Katsura, Y., Suenaga, A., Toyama, M., Terada, T., et al. (2007). Crystal structure of the rac activator, Asef, reveals its autoinhibitory mechanism. J Biol Chem 282, 4238-4242.

Nam, C. I., and Chen, L. (2005). Postsynaptic assembly induced by neurexinneuroligin interaction and neurotransmitter. Proc Natl Acad Sci USA 102, 61376142. 
Neeb, A. J. (2003). Neuroligin: Charakterisierung eines neuronalen Transmembranproteins. Dissertation: Mathematisch-naturwissenschaftliche Fakultäten, Georg-August-Universität Göttingen.

Nguyen, P. V., and Woo, N. H. (2003). Regulation of hippocampal synaptic plasticity by cyclic AMP-dependent protein kinases. Prog Neurobiol 71, 401-437.

Niell, C. M., Meyer, M. P., and Smith, S. J. (2004). In vivo imaging of synapse formation on a growing dendritic arbor. Nat Neurosci 7, 254-260.

Nyíri, G., Freund, T. F., and Somogyi, P. (2001). Input-dependent synaptic targeting of alpha(2)-subunit-containing $\mathrm{GABA}(\mathrm{A})$ receptors in synapses of hippocampal pyramidal cells of the rat. Eur J Neurosci 13, 428-442.

Papadopoulos, T., Korte, M., Eulenburg, V., Kubota, H., Retiounskaia, M., Harvey, R. J., Harvey, K., O'sullivan, G. A., Laube, B., Hülsmann, S., et al. (2007). Impaired GABAergic transmission and altered hippocampal synaptic plasticity in collybistindeficient mice. EMBO J.

Pfeiffer, F., Graham, D., and Betz, H. (1982). Purification by affinity chromatography of the glycine receptor of rat spinal cord. J Biol Chem 257, 9389-9393.

Pfrieger, F. W., and Barres, B. A. (1997). Synaptic efficacy enhanced by glial cells in vitro. Science $277,1684-1687$.

Poulopoulos A, Meyer G, Brose N, Varoqueaux F. (2008). Neuroligin 2 is a molecular organizer of the inhibitory postsynaptic scaffold. Submitted for publication.

Prange, O., Wong, T. P., Gerrow, K., Wang, Y. T., and El-Husseini, A. (2004). A balance between excitatory and inhibitory synapses is controlled by PSD-95 and neuroligin. Proc Natl Acad Sci USA 101, 13915-13920.

Prior, P., Schmitt, B., Grenningloh, G., Pribilla, I., Multhaup, G., Beyreuther, K., Maulet, Y., Werner, P., Langosch, D., and Kirsch, J. (1992). Primary structure and alternative splice variants of gephyrin, a putative glycine receptor-tubulin linker protein. Neuron 8, 1161-1170.

Proepper, C., Johannsen, S., Liebau, S., Dahl, J., Vaida, B., Bockmann, J., Kreutz, M. R., Gundelfinger, E. D., and Boeckers, T. M. (2007). Abelson interacting protein 1 (Abi-1) is essential for dendrite morphogenesis and synapse formation. EMBO J 26, 1397-1409.

R. Searle, J. o. h. n. (2005). Mind: A Brief Introduction. 240.

Rao, A., Cha, E. M., and Craig, A. M. (2000). Mismatched appositions of presynaptic and postsynaptic components in isolated hippocampal neurons. J Neurosci 20, 8344-8353.

Reagin, M. J., Giesler, T. L., Merla, A. L., Resetar-Gerke, J. M., Kapolka, K. M., and Mamone, J. A. (2003). TempliPhi: A sequencing template preparation procedure that eliminates overnight cultures and DNA purification. J Biomol Tech 14, 143-148. 
Reid, T., Bathoorn, A., Ahmadian, M. R., and Collard, J. G. (1999). Identification and characterization of hPEM-2, a guanine nucleotide exchange factor specific for Cdc42. J Biol Chem 274, 33587-33593.

Roberts, R. G., Freeman, T. C., Kendall, E., Vetrie, D. L., Dixon, A. K., ShawSmith, C., Bone, Q., and Bobrow, M. (1996). Characterization of DRP2, a novel human dystrophin homologue. Nat Genet 13, 223-226.

Roberts, R. G., and Sheng, M. (2000). Association of dystrophin-related protein 2 (DRP2) with postsynaptic densities in rat brain. Mol Cell Neurosci 16, 674-685.

Rosenmund, C., Rettig, J., and Brose, N. (2003). Molecular mechanisms of active zone function. Curr Opin Neurobiol 13, 509-519.

Ross, B. S., and Conroy, W. G. (2007). Capabilities of neurexins in the chick ciliary ganglion. Dev Neurobiol.

Rudolph, U., and Möhler, H. (2004). Analysis of GABAA receptor function and dissection of the pharmacology of benzodiazepines and general anesthetics through mouse genetics. Annu Rev Pharmacol Toxicol 44, 475-498.

Sabatini, D. M., Barrow, R. K., Blackshaw, S., Burnett, P. E., Lai, M. M., Field, M. E., Bahr, B. A., Kirsch, J., Betz, H., and Snyder, S. H. (1999). Interaction of RAFT1 with gephyrin required for rapamycin-sensitive signaling. Science 284, 1161-1164.

Saiyed, T., Paarmann, I., Schmitt, B., Haeger, S., Sola, M., Schmalzing, G., Weissenhorn, W., and Betz, H. (2006). Molecular basis of gephyrin clustering at inhibitory synapses: Role of G- and E-domain interactions. J Biol Chem.

Salinas, P. C., and Price, S. R. (2005). Cadherins and catenins in synapse development. Curr Opin Neurobiol 15, 73-80.

Sara, Y., Biederer, T., Atasoy, D., Chubykin, A., Mozhayeva, M. G., Südhof, T. C., and Kavalali, E. T. (2005). Selective capability of SynCAM and neuroligin for functional synapse assembly. J Neurosci 25, 260-270.

Saro, D., Li, T., Rupasinghe, C., Paredes, A., Caspers, N., and Spaller, M. R. (2007). A thermodynamic ligand binding study of the third PDZ domain (PDZ3) from the mammalian neuronal protein PSD-95. Biochemistry 46, 6340-6352.

Sassoè-Pognetto, M., Panzanelli, P., Sieghart, W., and Fritschy, J. M. (2000). Colocalization of multiple $\mathrm{GABA}(\mathrm{A})$ receptor subtypes with gephyrin at postsynaptic sites. J Comp Neurol 420, 481-498.

Sato, K., Sato, M., and Nakano, A. (1997). Rer1p as common machinery for the endoplasmic reticulum localization of membrane proteins. Proc Natl Acad Sci USA 94, 9693-9698.

Sato, K., Sato, M., and Nakano, A. (2001). Rer1p, a retrieval receptor for endoplasmic reticulum membrane proteins, is dynamically localized to the Golgi apparatus by coatomer. J Cell Biol 152, 935-944. 
Sato, K., Sato, M., and Nakano, A. (2003). Rer1p, a retrieval receptor for ER membrane proteins, recognizes transmembrane domains in multiple modes. Mol Biol Cell 14, 3605-3616.

Scheiffele, P., Fan, J., Choih, J., Fetter, R., and Serafini, T. (2000). Neuroligin expressed in nonneuronal cells triggers presynaptic development in contacting axons. Cell 101, 657-669.

Schweizer, C., Balsiger, S., Bluethmann, H., Mansuy, I. M., Fritschy, J. M., Möhler, $H$., and Lüscher, B. (2003). The gamma 2 subunit of $G A B A(A)$ receptors is required for maintenance of receptors at mature synapses. Mol Cell Neurosci 24, 442-450.

Semyanov, A., Walker, M. C., Kullmann, D. M., and Silver, R. A. (2004). Tonically active GABA A receptors: modulating gain and maintaining the tone. Trends Neurosci $27,262-269$.

Sgambato, A., and Brancaccio, A. (2005). The dystroglycan complex: from biology to cancer. J Cell Physiol 205, 163-169.

Sherman, D. L., Fabrizi, C., Gillespie, C. S., and Brophy, P. J. (2001). Specific disruption of a schwann cell dystrophin-related protein complex in a demyelinating neuropathy. Neuron 30, 677-687.

Sieghart, W., Fuchs, K., Tretter, V., Ebert, V., Jechlinger, M., Höger, H., and Adamiker, D. (1999). Structure and subunit composition of GABA(A) receptors. Neurochem Int 34, 379-385.

Sola, M., Bavro, V. N., Timmins, J., Franz, T., Ricard-Blum, S., Schoehn, G., Ruigrok, R. W., Paarmann, I., Saiyed, T., O'Sullivan, G. A., et al. (2004). Structural basis of dynamic glycine receptor clustering by gephyrin. EMBO J 23, 2510-2519.

Sola, M., Kneussel, M., Heck, I. S., Betz, H., and Weissenhorn, W. (2001). X-ray crystal structure of the trimeric N-terminal domain of gephyrin. J Biol Chem 276, 25294-25301.

Song, J. Y., Ichtchenko, K., Südhof, T. C., and Brose, N. (1999). Neuroligin 1 is a postsynaptic cell-adhesion molecule of excitatory synapses. Proc Natl Acad Sci USA 96, 1100-1105.

Songyang, Z., Blechner, S., Hoagland, N., Hoekstra, M. F., Piwnica-Worms, H., and Cantley, L. C. (1994). Use of an oriented peptide library to determine the optimal substrates of protein kinases. Curr Biol 4, 973-982.

Strochlic, L., Cartaud, A., and Cartaud, J. (2005). The synaptic muscle-specific kinase (MuSK) complex: new partners, new functions. Bioessays 27, 1129-1135.

Studler, B., Fritschy, J. M., and Brünig, I. (2002). GABAergic and glutamatergic terminals differentially influence the organization of GABAergic synapses in rat cerebellar granule cells in vitro. Neuroscience 114, 123-133. 
Studler, B., Sidler, C., and Fritschy, J. M. (2005). Differential regulation of GABA(A) receptor and gephyrin postsynaptic clustering in immature hippocampal neuronal cultures. J Comp Neurol 484, 344-355.

Sumita, K., Sato, Y., lida, J., Kawata, A., Hamano, M., Hirabayashi, S., Ohno, K., Peles, E., and Hata, Y. (2006). Synaptic scaffolding molecule (S-SCAM) membrane-associated guanylate kinase with inverted organization (MAGI)-2 is associated with cell adhesion molecules at inhibitory synapses in rat hippocampal neurons. J Neurochem 100, 154-166.

Sur, C., Wafford, K. A., Reynolds, D. S., Hadingham, K. L., Bromidge, F., Macaulay, A., Collinson, N., O'Meara, G., Howell, O., Newman, R., et al. (2001). Loss of the major $\operatorname{GABA}(\mathrm{A})$ receptor subtype in the brain is not lethal in mice. J Neurosci 21, 3409-3418.

Tabuchi, K., Blundell, J., Etherton, M. R., Hammer, R. E., Liu, X., Powell, C. M., and Südhof, T. C. (2007). A Neuroligin-3 Mutation Implicated in Autism Increases Inhibitory Synaptic Transmission in Mice. Science.

Takeichi, M. (2007). The cadherin superfamily in neuronal connections and interactions. Nat Rev Neurosci 8, 11-20.

Temburni, M. K., Rosenberg, M. M., Pathak, N., McConnell, R., and Jacob, M. H. (2004). Neuronal nicotinic synapse assembly requires the adenomatous polyposis coli tumor suppressor protein. J Neurosci 24, 6776-6784.

Tretter, V., Jacob, T. C., Mukherjee, J., Fritschy, J. M., Pangalos, M. N., and Moss, S. J. (2008). The clustering of $\operatorname{GABA}(\mathrm{A})$ receptor subtypes at inhibitory synapses is facilitated via the direct binding of receptor alpha2 subunits to gephyrin. J Neurosci 28, 1356-1365.

Ullian, E. M., Christopherson, K. S., and Barres, B. A. (2004). Role for glia in synaptogenesis. Glia 47, 209-216.

Ullrich, B., Ushkaryov, Y. A., and Südhof, T. C. (1995). Cartography of neurexins: more than 1000 isoforms generated by alternative splicing and expressed in distinct subsets of neurons. Neuron 14, 497-507.

Varoqueaux, F., Aramuni, G., Rawson, R. L., Mohrmann, R., Missler, M., Gottmann, K., Zhang, W., Südhof, T. C., and Brose, N. (2006). Neuroligins determine synapse maturation and function. Neuron 51, 741-754.

Varoqueaux, F., Jamain, S., and Brose, N. (2004). Neuroligin 2 is exclusively localized to inhibitory synapses. Eur J Cell Biol 83, 449-456.

Varoqueaux, F., Sigler, A., Rhee, J. S., Brose, N., Enk, C., Reim, K., and Rosenmund, C. (2002). Total arrest of spontaneous and evoked synaptic transmission but normal synaptogenesis in the absence of Munc13-mediated vesicle priming. Proc Natl Acad Sci USA 99, 9037-9042.

Vaughan, K. T. (2005). TIP maker and TIP marker; EB1 as a master controller of microtubule plus ends. J Cell Biol 171, 197-200. 
Vojtek, A. B., Hollenberg, S. M., and Cooper, J. A. (1993). Mammalian Ras interacts directly with the serine/threonine kinase Raf. Cell 74, 205-214.

Wallar, B. J., and Alberts, A. S. (2003). The formins: active scaffolds that remodel the cytoskeleton. Trends Cell Biol 13, 435-446.

Wang, H., Bedford, F. K., Brandon, N. J., Moss, S. J., and Olsen, R. W. (1999). $\operatorname{GABA}(\mathrm{A})$-receptor-associated protein links $\mathrm{GABA}(\mathrm{A})$ receptors and the cytoskeleton. Nature 397, 69-72.

Waters, J., Schaefer, A., and Sakmann, B. (2005). Backpropagating action potentials in neurones: measurement, mechanisms and potential functions. Prog Biophys Mol Biol 87, 145-170.

Wojcik, S. M., and Brose, N. (2007). Regulation of membrane fusion in synaptic excitation-secretion coupling: speed and accuracy matter. Neuron 55, 11-24.

Wojcik, S. M., Katsurabayashi, S., Guillemin, I., Friauf, E., Rosenmund, C., Brose, N., and Rhee, J. S. (2006). A shared vesicular carrier allows synaptic corelease of GABA and glycine. Neuron 50, 575-587.

Xiang, S., Kim, E. Y., Connelly, J. J., Nassar, N., Kirsch, J., Winking, J., Schwarz, G., and Schindelin, H. (2006). The crystal structure of Cdc42 in complex with collybistin II, a gephyrin-interacting guanine nucleotide exchange factor. J Mol Biol 359, 35-46.

Xue, H. (1998). Identification of major phylogenetic branches of inhibitory ligandgated channel receptors. J Mol Evol 47, 323-333.

Yu, W., Charych, E. I., Serwanski, D. R., Li, R. W., Ali, R., Bahr, B. A., and De Blas, A. L. (2008). Gephyrin Interacts With The Glutamate Receptor Interacting Protein 1 Isoforms At GABAergic Synapses. J Neurochem.

Zhorov, B. S., and Tikhonov, D. B. (2004). Potassium, sodium, calcium and glutamate-gated channels: pore architecture and ligand action. J Neurochem 88 , 782-799.

Zhou, F. X., Cocco, M. J., Russ, W. P., Brunger, A. T., and Engelman, D. M. (2000). Interhelical hydrogen bonding drives strong interactions in membrane proteins. Nat Struct Biol 7, 154-160.

Zoghbi, H. Y. (2003). Postnatal neurodevelopmental disorders: meeting at the synapse? Science 302, 826-830. 


\section{About the Author}

The author was born on May 6 $6^{\text {th }}, 1980$ in Athens, Greece. In 1985 he moved with his parents to Ithaca, NY where he attended Bell Sherman Elementary and Boynton and Dewitt Middle schools. In 1993 he moved to Grenoble, France attending the collège of the École Internationale Stendhal, and in 1994 moved back to his hometown studying at the Gymnasium Palinostouton Hellenopedon of Hellenicon and the $2^{\text {nd }}$ Gymnasium of Alimos. He graduated from the Eniaio Polykladic Lyceum of Alimos and was admitted to the School of Biology of the National and Kapodistrian University of Athens on a scholarship from the Greek State Scholarships Foundation in 1998. He graduated in 2003 after completing his Diploma thesis in the lab of Dr. Spiros Efthimiopoulos in the Department of Animal and Human Physiology. The same year he was admitted to the MSc/PhD/MD-PhD Program Neurosciences of the Georg-August University of Göttingen, Germany. In 2004, being a fellow of the German Research Foundation graduate program for "ProteinProtein Interactions During Intracellular Transport of Macromolecules", he started work on his doctoral thesis under the supervision of Dr. Frédérique Varoqueaux in the lab of Prof. Nils Brose in the Max Planck Institute of Experimental Medicine in Göttingen. 


\section{List of Publications}

Poulopoulou C, Markakis I, Davaki P, Nikolaou C, Poulopoulos A, Raptis E, Vassilopoulos D. (2005). Modulation of voltage-gated potassium channels in human Tlymphocytes by extracellular glutamate. Molecular Pharmacology 67, 856-867.

Fotinopoulou A, Tsachaki M, Vlavaki M, Poulopoulos A, Rostagno A, Frangione B, Ghiso J, Efthimiopoulos S. (2005). BRI2 interacts with APP and regulates Abeta production. Journal of Biological Chemistry 280, 30768-30772.

Aramuni G, Varoqueaux F, Poulopoulos A, Brose N, Zhang W. (2008). Neuroligin 2 Regulates the Maturation and Function of GABAergic and Glycinergic Synapses. Submitted for publication.

Poulopoulos A, Meyer G, Brose N, Varoqueaux F. (2008). Neuroligin 2 is a molecular organizer of the inhibitory postsynaptic scaffold. Submitted for publication. 